

\section{DISCLAIMER}

This report was prepared as an account of work sponsored by an agency of the United States Government. Neither the United States Government nor any agency Thereof, nor any of their employees, makes any warranty, express or implied, or assumes any legal liability or responsibility for the accuracy, completeness, or usefulness of any information, apparatus, product, or process disclosed, or represents that its use would not infringe privately owned rights. Reference herein to any specific commercial product, process, or service by trade name, trademark, manufacturer, or otherwise does not necessarily constitute or imply its endorsement, recommendation, or favoring by the United States Government or any agency thereof. The views and opinions of authors expressed herein do not necessarily state or reflect those of the United States Government or any agency thereof. 


\section{DISCLAIMER}

Portions of this document may be illegible in electronic image products. Images are produced from the best available original document. 
THIS PAGE

\section{WAS INTENTIONALLY LEFT BLANK}




\section{THIS PAGE}

\section{WAS INTENTIONALLY \\ LEFT BLANK}


AN EXPERIMENTAL INVESTIGATION OF THE TRAPPING ENERGY LOSS MECHANISMS OF INTENSE RELATIVISTIC ELECTRON RINGS IN HYDROGEN GAS AND PLASMA*

Adrian Charles Smith, Jr.

\section{ABSTRACT}

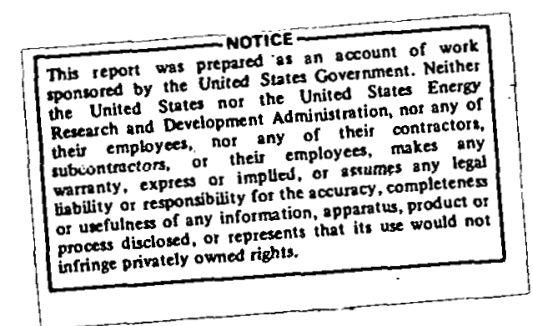

The results of an experimental study on the trapping and energy loss mechanisms of intense, relativistic electron rings confined in Astron-like magnetic field geometries are presented. The work is subdivided into four sections: gas trapping; average ring electron energetics; plasma trapping, and hollow-beam cusp-injection into gas and plasma.

The mechanisms by which the injected beam coalesces into a current ring in the existing cornell RECEBerta facility are considered first. Data from an axial. array of magnetic probes is compared with findings from a high-energy electron current monitor to show that the

*This work was begun under ERDA contract No. E(11-1)-2319 and was completed under ERDA contract No. W-7405-Eng-48. 
trapping proceeds as a relaxation process in which an initially-uniform, circulating sheath of current collapses axially in 300-500 ns to form a steady-state ring. This process is analyzed in terms of a simple model involving the tensor conductivity of the beam-created plasma and an electric circuit analogy which show that the relaxation proceeds on a time scale consistent with the magnetic field penetration time calculated for a rotating cylinder of current flowing in a resistive background medium. To investigate the nature of ring electron energy loss mechanisms following completion of the trapping process, a diagnostic was developed utilizing multi-foil $\mathrm{X}$-ray absorption spectroscopy to analyze the Bremsstrahlung generated by the electrons as they impinge upon a thin tungsten wire target suspended in the circulating current. The ring energetics could be varied either by altering the perturbing effects of the wire target or the composition of the background gas. The experimental results showed that the particle energy loss rates were constant functions of time and that, typically, net energy losses of about $10 \%$ of the initial ring energy (500keV) were sustained by the electrons during their residence in the mirror trap. The energy loss mechanisms were analyzed classically by considering (i) collisional energy losses. 
in the background gas and (ii) the betatron acceleration of ring electrons due to magnetic flux changes within the circulating ring. Good agreement was obtained between the experimental results and the calculations.

The results of electron ring trapping and confinement in neutral gas were extended to studying these processes in a preionized plasma. A magnetoplasmadynamic arcjet and capacitor bank pulse-line were developed to preionize the interior of RECE-Berta to densities of $n_{p}$. $10^{12} \mathrm{~cm}^{-3}\left(\mathrm{n}_{\text {BEAM }} \sim 10^{9} \mathrm{~cm}^{-3}\right)$. At plasma densities of $\mathrm{n}_{\mathrm{p}}>5-8 \times 10^{11} \mathrm{~cm}^{-3}$, the beam was found to "stall" near the point of injection into the mirror trap. Lowering the plasma density into the range $5 \times 10^{11} \mathrm{~cm}^{-3}<\mathrm{n}_{\mathrm{p}}<5 \times 10^{10}$ : $\mathrm{cm}^{-3}$ resulted in the beam's moving slowly--as a ring--into the minimum of the mirror trap, where weak $(\zeta<30 \%)$ rings having lifetimes of up to 350-400 $\mathrm{\mu s}$ were obtained. A comparison of these observations with the theoretical treatment by Chu and Rostoker is discussed.

Finally, a set of preliminary experimental results is presented in which an annular electron beam was passed through a coaxial, non-adiabatic magnetic cusp located at one end nf a magnetic mirror well. These experiments represent an extension of the work of Kribel, et al., to include ring confinement in puffed, low-pressure hydrogen 
gas backfills and preionized hydrogen plasma. The strong $(\zeta \sim 80 \%)$ rings thus confined were observed to have linear decay characteristics which scaled from the Berta experiments, with lifetimes of up to $80 \mu \mathrm{s}$ obtained in the gas backfill. Somewhat weaker $(\zeta \sim 70 \%)$ rings lasting up to $140 \mu \mathrm{s}\left(2 \times 10^{5}\right.$ gyro-orbits) were maintained in the preionized background.

\section{ACKNOWLEDGMENTS}

The author is indebted to many individuals for their encouragement and assistance throughout the course of this work.

The program of research was conducted under the guidance of Professor Hans H. Fleischmann. His extensive physical insight and detailed scrutiny of the experimental work were almost always helpful in formulating self-consistent explanations of the phenomena observed during these experiments.

I would like to thank Dr. Clarke E. Swannack for his help with, and many contributions to, the energy measurement diagnostic. It was through his efforts that a1most all of the operational mechanics of the Monte Carlo computations were properly carried out at Los Alamos. 
Throughout the experimental work, I had many very instructive and stimulating discussions with Dr. David L. Morse and Dr. Bruce R. Kusse, of the Laboratory of Plasma Studies at Cornell University. Both individuals freely contributed their time to discuss various aspects of the experiments with me: Dr. Morse largely in the arcjet/pulseline design and Dr. Kusse primarily in the cusp experiment and slotted-cusp concept. Their generous assistance is gratefullỳ acknowledged.

The Monte Carlo calculations described in Chapter 4 were performed through the courtesy and guidance of Dr. R. G. Schrandt, Dr. L. Carter, and Dr. W. L. Thompson, all of the Los Alamos Scientific Laboratory. In particular, I wish to express my appreciation to Dr. Thompson for his efforts in adapting the Bremsstrahlung spectrum codes to the particular target geometry unique to this experimental work.

I would like to express my gratitude to Professor R. N. Sudan for his thoughtful comments and his continued interest in the progress of this research.

I would also like to thank Dr. David A. Hammer of the Naval Research Laboratory for kindly offering the use of the electron beam return-current/plasma interaction code calculations pertaining to results discussed in Chapter 3 . 
My constant interaction with the other members of the Cornell Relativistic Electron Coil Group, Dr. J. J. Bzura, Dr. H. A. Davis, S. C. Luckhardt, R. A. Meger, and Dr. D. M. Woodall, as well as with Dr. Carl A. Ekdahl and Dr. John D. Sethian of the Plasma Turbulent Heating group, is happily acknowledged, since our common research interests often led to the exchange of helpful and instructive ideas. The large array of oscilloscopes, the $140 \mathrm{GH} z$ microwave interferometer, and a varied assortment of other necessary hardware was made available to me from the Lawrence Livermore Laboratory through the very generous efforts of Dr. W. C. Condit, Jr. Guidance in the use of the interferometer hardware was provided by Dr. Donald Meeker, and assistance in constructing the arcjet pulse-line was supplied by Mr. D. Trimble, both of the Lawrence Livermore Laboratory. Many of the experiments carried out in this work would not have been possible without this support equipment. Finally, I would like to extend my thanks to $\mathrm{Mr}$. Frank Redder and Mr. Jim Ivers of the Cornell High Voltage Laboratory for their constant, competent assistance in helping me maintain the electron beam facility in operating condition during these experiments. 
TABLE OF CONTENTS

Page

CHAPTER 1

INTRODUCTION. . . . . . . . . . .

1.1 The Astron ............ 2

1.2 The Cornell Relativistic Electron Coil Program . . . . . . . . . . 9

1.3 Objectives of the Dissertation. . . 16

1) Ring Trapping in Hydrogen Gas. • 17

2) Average Ring Electron Energy Measurement. . . . ... . 21

3) Electron Beam Trapping and Confinement in Hydrogen Plasma. . . 22

4) Cusp-Injection . . . . . . . 24

1.4 Publications and Presentations Related to this Work. ......... 25

\section{CHAPTER 2}

EXPERIMENTAL APPARATUS. . . . . . . . . 29

2.1 Introduction. . . . . . . . 30

2.2 QWIBLE. . . . . . . . . 30

2.3 RECE-Berta . . . . . . . . . 31 
Page

CHAPTER 3

SUB-MICROSECOND TRAPPING OF ELECTRON RINGS IN RECE-BERTA IN NEUTRAL GAS. . . . . . . . . . . 43

3.1 Introduction .......... 44

3.2. . Experimental Diagnostics. . . . 45

3.2.1 Plasma Density Meașurements . . . 48

3.2.1.1 Interferometer Configuration. . . 49

3.2.1.2 Experimental System Tests . . . 53

3.2.2 Magnetic Field Probes . . . . . 54

3.2.2.1 Magnetic Probe Design . . . . 54

3.2.2.2 Physical Construction . . . . 58

3.2.3 High-Energy Electron Current

Monitor ......... . . 60

3.2.3.1 Target Geometry and configuration 61

3.2.3.2 Detector Configuration. . . . . 63

3.2.3.3 Detector Calibration . . . . 68

3.2.3.4 Detection Statistics . . . . . 76

3.3 Experimental Trapping Results . - 77

3.3.1 Plasma Density Measurements . . . 77

3.3.2 Diamagnetic Field Measurements. • 87

3.3.3 High-Energy Electron Current

Monitor . . . . . . . . 102 
Page

3.4 Interpretation of the Experimental Results. . . . . . . . . 115

3.4.1 A Physical Model for the Trapping Process . . . . . . . . . 116

3.4.2 Energy Deposition in the Sheath Plasma . . . . . . . . . 135

3.5 Summary and Discussion . . . . 139 CHAPTER 4

AVERAGE RING ELECTRON ENERGY MEASUUREMENTS • • • 146 4.1 . Introduction - . . . . . . . . 147

4.2 The Experimental Technique . . . 149

4.3 Experimental Arrangement . . . . 157

4.3.1 Operating Parameters of RECE-

4.3.2 Bremsstrahlung Sourge Target . . 160

4.3.3 Multi-Channel Detector Configuration . . . . . . . . . 162

4.4 Theory of Multi-Channel X-ray Absorption Spectroscopy. . . . 166 4.4.1 Detector Output Signals: A General Expression . . . . . 166 4.4.2 Evaluation of the Bremsstrahlung Source Term. . . . . . . . . 173 
4.4.2.1 Description of the Monte Carlo Transport Codes. . . . . . . 174

4.4.2.2 Angular Dependences of the Bremsstrahlung Spectra . . . . 177

4.4.3 Absorber Transmission Factors . 198

4.4.3.1 Absorber Transmission Coefficients. . . . . . . . 202

4.4.4 Sodium Iodide Detector Efficiencies. . . . . . . . . . 206

4.4.5 Energy Dependence of the Detector Signal Ratios. . . . . . . 211

4.4.6 Code Error Analysis. . . . . . 214

4.4.7 Energy Resolution ....... 217

4.5 Experimental Procedures. . . . 223

4.5.1 Alignment and Collimation Tests. 223

4.5.2 Detector Calibration . . . . 224

4.6 Average Ring Electron Energy

Measurements . . . . . . 235

4.6.1 Variation of Machine Parameters. 235

4.6.2 Variation of Wire Perturbation . 236

4.6.3 Sumary of Experimental Results. 239

4.7 Experimental Error Analysis. • 259

4.8 Average Ring Electron Energy

Loss Rate Analysis . . . . . 262 
Page

4.8.1 Electron Energy Loss Rates. .. . . 262

4.8.2 Betatron Acceleration .. . . . 264

4.8.3 Summary of the Experimental and

Computational Results . . . . . 273

4.9 Summary and Discussion of the

Results . . . . . . . . . . 278

4.9.1 Discussion of the Experimental

Findings . . . . . . . . . 278

4.9.2 Comparison with Theoretical

Analyses of Microinstabilities. . . 281

4.10 Extension and Application of

Energy Measurement to Nanosecond

Time Scales . . . . . . . . 289

\section{CHAPTER 5}

TRAPPING AND CONFINEMENT OF ELECTRON RINGS IN A PREIONIZED MEDIUM. • . . . . . . . . . . . • 302

5.1 Introduction ... . . . . . 303

5.2 Experimental Arrangement. . . . . 306

5.2.1 The Plasma Gun . . . . . . . 306

5.2.2 Pulse-Line Design and Construction. 315

5.2.3 Plasma Source Operation . . . . 326

5.2.4 Graded-Field Structures . . . . 329 
5.2.5 Plasma Diagnostics. .. . . . . : 335

5.2.5.1 Plasma Density Measurements . . . 336

5.2.5.2 Plasma Reproducibility and Noise. 351

5.2.5.3 Plasma Currents and Self-Magnetic Fields. . . . . . . . . . 354

5.2.6 Experimental Diagnostics. . . . 357

5.2.6.1 Magnetic Probes . . . . . . . 360

5.2.6.2 Fast Electron Current Monitor • . 361

5.2.7 Experimental Timing Sequence. . . 361

5.3 Experimental Results . . . . . 361

5.3.1 Diamagnetic Field Profiles. . . . 361

5.3.2 High-Energy Electron Current

Observations. . . . . . . . 381

5.3.3 Trapping of Electron Rings sans

Quadrupole Stabilization. . . . 398

5.3.4 Long-Term Electron Ring Confinement in a Background Plasma . . . . . 401

5.4 Plasma-Trapping and the Model of Chu and Rostoker. . . . . . . . 405

5.4.1 Description of the Theoretical Múdel . . . . . . . . . . 406

5.4.2 Comparison with Experimental Results . . . . . . . . . . 
5.4.3 Limitations of the Theory.....

\section{CHAPTER 6}

THE CONFINEMENT OF CUSP-INJECTED, RELATIVISTIC

ELECTRON RINGS IN NEUTRAL GAS ANd PLASMa. • • • 436

6.1 Introduction ........... 437

6.2 The Basic Principles of cusp-

Injection. . . . . . . . . 439

6.3 Experimental Cusp-Injection

Apparatus. . . . . . . . . 451

6.3.1 Confinement Tank and vacuum

Systems ........... . 456

6.3.2 Magnetic Field Structures. . . 457

6.3.3 Beam Injection Structure . . . . 461

6.3.4 Plasma Source. . . . . . . . 463

6.3.5 Diagnostics .......... 463

6.3.5.1 Magnetic Probes.......... 463

6.3.5.2 X-ray Monitor. . . . . . . . 468

6.4 Puffed-Neutral Gas Confinement of

Electron Rings ........ . 468

6.4.1 Transient Gas Density Measurements 469

6.4.2 Experimental Results ...... 473 
6.5 Confinement of Cusp-Injected

Beams in a Preionized Medium. . . 483

6.5.1 Plasma Density Measurements . . . 483

6.5.2 Experiments and Results..... 485

6.6 Discussion and Summary . . . . 491

\section{APPENDICES}

APPENDIX I . PLASMA DENSITY MEASUREMENTS VIA

MICROWAVE INTERFEROMETRY •. . . 496

I.1 Partial Fringe-Shift Data Analysis. 496

I.2 Plasma Density Measurements in the

Cusp-Injected Device. ...... 500

APPENDIX II PHOTON MASS-ATTENUATION COEFFICIENTS

FOR NaI, $\mathrm{Sn}, \mathrm{Pb}, \mathrm{AND} \mathrm{Cu}$.... 509

APPENDIX III MONTE CARLO CODE CALCULATIONS • . 518

III.1 Summary of Transport Code Inter-

actions . . . . . . . . 518

I. Electron Interactions .... 518

III. Photon Interactions .... 521

III.2 Operational Use of Transport codes. 523

III.3 The wire Target Geometry. . . . . 531 
III. 4 Code Output for Absorber Trans-

mission Coefficients, $\operatorname{NaI(TI)~and~}$

Pilot B Detector Efficiencies, and

Detector Signal Ratios as Functions

of Electron Energy. . . . . . . 539

III.5 Bremsstrahlung code Output:

Electrons Incident on Tungsten Slab and Wire Geometry Targets, $100 \mathrm{keV}$ $<E_{0}<1000 \mathrm{keV} \cdot . . . . . . .549$

III.6 An Estimate of the Compton Effect in the Absorption Foils.... . 575

III.7 A Simple Model for Estimating the Sodium Iodide Detector Efficiency : 588

APPENDIX IV PREDICTED X-RAY DETECTOR OUTPUT SIGNALS ' . . . . . . . . . 597

IV.1 Sodium Iodide Detector. . . . . 597 IV.2 Pilot B Detector. . . . . . . 603

APPENDIX $V$ DETAILS OF ARCJET OPERATING CHARACTERISTICS . . . . . . . . . 608

I. Construction .......... 608

II. Valve Characteristics . . . . 608

III. Electrical Characteristics. . . . 609 
APPENDIX VI PULSED-NEUTRAL GAS DENSITY

MEASUREMENTS. . . . . . . . 612

BIBLIOGRAPHY ................. 624

xviii 


\section{LIST OF TABLES}

Page

\section{CHAPTER 3}

\section{Table 3-1}

Circulating Plasma Current Decay Times. . . . . . 134

\section{CHAPTER 4}

Table 4-1

Results of Experimental Measurements of dU/dt . . 260 Table 4-2

Corrected Gasfill Partial Pressures and Fast. Electron Energy Loss Rates. . . . . . . . . 265 Table 4-3

Sensitivity of Calculated dU/dt Values to Ring

Parameter variations. . . . . . . . . . . . 274

Table 4-4

Summary of Ring Electron Energy Decay Rates . - . 275.

Table 4-5

Predicted Ring Electron Energy Spreads. . . . : 277

Table 4-6

Summary of Hybrid Mode Collisional stabilization

criteria. ... .... . . . . . . . . . 285

Table 4-7

Summary of Hybrid Mode Energy Spread Stabilization Criteria . . . . . . . . . . . . . . 288 


\section{CHAPTER 5}

Table 5-1

Summary of Arcjet Plasma and Injected Electron

Beam Parameters. . . . . . . . . . . . .

\section{APPENDICES}

APPENDIX III

Table III-1

Absorber+Collimator Transmission Coefficient .

Table III-2

NaI Crystal Detection Efficiency Arrangement .

Table III-3

Gamma Transmission from 100 to $1000 \mathrm{KeV}, 12.5 \mathrm{keV}$

Steps for $1,2,3$, and $0 \mathrm{~Pb}$ Absorber Combinations.

Table III-4

NaI(TI) Crystal Efficiency from 100 to $1000 \mathrm{keV}$,

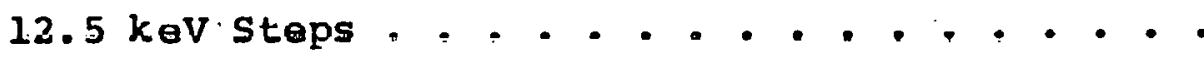

Table III-5

Pilot B Crystal Efficiency from 100 to $1000 \mathrm{keV}$, $12.5 \mathrm{keV}$ Steps . . . . . . . . . . . .

Table III-6

NaI(T1) - Intensity Ratios of Bremsstrahlung into the Detector as a Function of Absorber and Initial Electron Energy. . . . . . . . . 
Table III-7

Pilot B - Intensity Ratios of Bremsstrahlung into the Detector as a Function of Absorber and Initial Electron Energy . . . . . . . .

Table III-8

Energy Loss Rate, Range, and Bremsstrahlung

Yield for Electrons on Tungsten $\left(10 \mathrm{keV}<\mathrm{E}_{\mathrm{o}}<\right.$

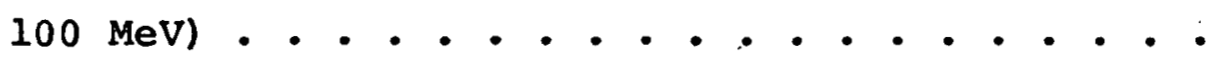

Table III-9

Summary of Monte Carlo Computed Bremsstrahlung

Spectra, $100 \mathrm{keV}<\mathrm{E}_{\mathrm{o}}<1000 \mathrm{keV}$. . . . . .

Table III-10

Monte Carlo Computed Bremsitrahlung Spectra for Electrons Incident on a $2.54 \times 10^{-3} \mathrm{~cm}$ diam Tungsten Wire. . . . . . . . . . . . . 576 Table III-II

The Quantity "F" vs Slab Thickness . . . . . . 587

APPENDIX IV

Table IV-1

Bremsstrahlung spectra as seen by the Detector System for Each of $3 \mathrm{~Pb}$ Absorber Combinations as

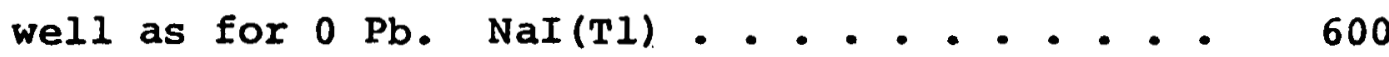


Page

Table IV-2

Bremsstrahlung Spectra as seen by the Detector

System for each of $3 \mathrm{~Pb}$ Absorber Combinations

as well as for $0 \mathrm{~Pb}$. Pilot $\mathrm{B}$. . . . . . 


\section{LIST OF FIGURES}

Page

\section{CHAPTER 1}

1-1 Conceptual view of an electron ring confined in a magnetic mirror well.. . .

1-2. Scale comparison between the Astron and RECE-Berta . . . . . . . . .

1-3 (Top): Time-integrated photograph of electron ring trapped in RECE-Berta (photo taken from upstream end of Berta); (Bottom): Typical electron ring diamagnetic signals showing trapping, linear decay, and "dump" features . . . . .

1-4 Position of the peak current density of an E-layer confined in the Astron as a function of time. The curve approximates the position of the center of the circulating charge distribution. (Beam energy = $5.8 \mathrm{MeV}$, Beam current $=200 \AA$, Mirror depth $=$ 2\%, Hydrogen pressure $=17$ mTorr). (From Reference 19) . . . . . . . . 19 
CHAPTER 2

2-1 QWIBLE electron beam diode voltage and current waveforms. . . . . . . . . 33

2-2 Schematic illustration of RECE-Berta . . . 36

2-3 (Top): Typical axial magnetic field profile in RECE-Berta; (Lower Left): Electrical schematic of the pulsed magnetic field circuit for RECE-Berta; (Lower Right): Typical pulsed-field current waveform • .

2-4 Photograph of the RECE-Berta facility.

Shown are the Marx-generator, QWIBLE, RECEBerta, the cusp-injection device (cf. Chapter 6) in the foreground, and the $\mathrm{x}$-ray multi-channel absorption spectrograph to the left of Berta (cf. Chapter 4). . .

\section{CHAPTER J}

3-1 Diagnostic arrangement used for fasttrapping studies... . . . . . . . .

3-2 Spatial configuration of $2 \mathrm{~mm}$ Mach-Zehnder interferometer with respect to confined electron ring. . . . . . . . . . . . 
3-3 (TOp): Details of magnetic pickup probe construction; (Bottom): Electrical schematic of probe integrating circuitry. . . . . . . . . . . . . 56

3-4 Collimation geometry for high-energy electron current monitor. . . . . . . . . 65

3-5 Detector current waveforms resulting from full-body irradiation of Pilot B detector by $\mathrm{Co}^{60}$ source; time of photograph exposure $=12 \mathrm{sec} . . . . . . . . . . . . .470$

3-6 Gain-saturation tests on fast $\mathrm{x}-\mathrm{ray}$ detector. . . . . . . . . . . . .

3-7 $x$-ray detector current versus axial diamagnetic field signals for confined rings on-center with the tungsten target as a function of time. Individual shot numbers are indicated . . . . . . . . . . . .

3-8 Characteristic interferometer responses:

(Top) : without a downstream mirror, i.e., no confined ring, single pass of spiral electron beam; (Center): with a strong ( $\zeta \sim 75 \%)$ confined electron ring;

(Bottom): with a confined ring and initial phase of interferometer advanced by about $270^{\circ}$ 
Page

3-9 Integrated plasma line density as a

function of time for various confined

electron rings as inferred from inter-

ferometer data. . . . . . - . .

83

3-10 Fast-rising interferometer response, indi-

cating probable passage of injected beam

between interferometer horns. . . . .

86

3-11 Sample oscilloscope traces from magnetic

probe array and reduced data parameterized to time after beam injection, for electron

ring confined in 910 mTorr hydrogen . .

3-12 Axial diamagnetic profile for the ring

trapping process in 150 mTorr hydrogen. .

3-13 Axial diamagnetic profile for the ring

trapping process in 340 mTorr hydrogen. .

3-14 Axial diamagnetic profile for the ring

trapping process in 600 mTorr hydrogen. .

3-15 Axial diamagnetic profile for the ring

trapping process in 800 mTorr hydrogen. . 101

3-16 Direct comparison of magnetic pickup

probe signals with the $x$-ray detector

responses for injected currents at

various axial positions in Berta.... . 104 
3-17 Comparison of axial diamagnetic field profiles with circulating current profiles as a function of time for the ring trapping process in 150 mTorr hydrogen. . . . . . . . . 108

3-18 Comparison of axial diamagnetic field profiles with circulating current profiles as a function of time for the ring trapping process in 340 mTorr hydrogen. . . . . . . . . . 110

3-19 Comparison of axial diamagnetic field profiles with circulating current profiles as a function of time for the ring trapping process in 850 mTorr hydrogen. : . 112

3-20 Configuration of beam-created plasma channel in Berta (cross-sectional view) - 120

\section{CHAPTER 4}

4-1 Ideal absorption spectroscopy experiment. . . . . . . . . . . . 152

4-2 Conceptual illustration of energy measurement diagnostic . . . . . . . . 155

4-3 Layout of the energy measurement diagnostic. 159 
Page

4-4 Illustration of source, absorber, collimator, and detector geometries for use in Equations $(4.2)$ to $(4.4) \ldots . . . .$. 169

4-5 Comparison of the angular distribution of Bremsstrahlung from a $1.52 \times 10^{-3} \mathrm{~cm}$ tungsten slab with that of a $2.54 \times 10^{-3} \mathrm{~cm}$ tungsten slab; $E_{0}=150 \mathrm{kev} . . . . . . . .180$ 4-6 Angular distribution of Bremsstrahlung from a $1.52 \times 10^{-3} \mathrm{~cm}$ tungsten slab; $E_{0}=300 \mathrm{keV} .182$ 4-7 Angular distribution of Bremsstrahlung from a $1.52 \times 10^{-3} \mathrm{~cm}$ tungsten slab; $\mathrm{E}_{0}=500 \mathrm{keV} . \quad 184$ 4-8 Angular distribution of Bremsstrahlung from a $1.52 \times 10^{-3} \mathrm{~cm}$ tungsten slab; $\mathrm{E}_{0}=700 \mathrm{keV} .186$ 4-9 Angular distribution of Bremsstrahlung from a $1.52 \times 10^{-3} \mathrm{~cm}$ tungsten slab; $\mathrm{E}_{0}=1000 \mathrm{keV} .188$ 4-10 Angular distribution of Bremsstrahlung from a $2.54 \times 10^{-3} \mathrm{~cm}$ tungsten slab; $\mathrm{E}_{0}=300 \mathrm{keV} .190$ 4-11 Angular distribution of Bremsstrahlung from a $2.54 \times 10^{-3} \mathrm{~cm}$ tungsten slab; $\mathrm{E}_{0}=500 \mathrm{keV} .192$ 4-12 Angular distribution of Bremsstrahlung from a $2.54 \times 10^{-3} \mathrm{~cm}$ tungsten siab; $\mathrm{E}_{0}=700 \mathrm{kev} .194$ 4-13 Angular distribution of Bremsstrahlung from a $2.54 \times 10^{-3} \mathrm{~cm}$ tungsten slab; $\mathrm{E}_{0}=1000 \mathrm{kev} .196$ 
Page

4-14 Bremsstrahlung spectra from a $2.54 \times 10^{-3} \mathrm{~cm}$ diameter tungsten wire, integrated over the

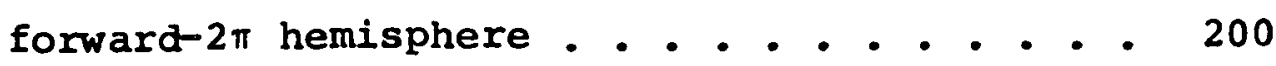

4-15 Absorber transmission coefficients for " 0 " , "l", "2", and "3" $\mathrm{Pb} / \mathrm{Sn}$ absorbers . . . . 205

4-16 X-ray detection efficiency for a rightcylindrical sodium Iódide crystal $4.5 \mathrm{~cm}$ in diameter, $5.1 \mathrm{~cm}$ long . . . . . . . .

4-17 Families of curves depicting NaI(TI) detector signal ratios as a function of primary electron energy parameterized to the various thicknesses of absorber used in the experiment. - . . . . . . . . . . . . .

4-18 Primary electron energy as predicted by various NaI(T1) detector signal ratios having different absorber combinations for a monodirectional and diffuse electron beam . . .

4-19 X-ray energy calibration of NaI(Tl) X-ray detectors . . . . . . . . . . . . 227

4-20 NaI (TI) detector response to $\mathrm{Co}^{60}$ and $\mathrm{Na}^{23}$. 230 4-21 X-ray detector response with tungsten wire target suspended in, and removed from, the ring confinement region . . . . . . . . 233 
4-22 Location of tungsten wire target with respect to the ring diamagnetic profile for the two target positions studied in this work. . . : . . . . . . . . . .

4-23 Characteristic signals from three-channel $x$-ray detector and axial magnetic probes for an electron ring confined in a backfill of 450 mTorr hydrogen; target located at axial center of ring current distribution. .

4-24 Characteristic signals from three-channel $\mathrm{x}$-ray detector and axial magnetic probes for an electron ring confined in a backfill of 450 mTorr hydrogen; target located at edge of ring current distribution. . : . .

4-25 Characteristic signals from three-channel $x$-ray detector and axial magnetic probes for an electron ring confined in a backfill of 750 mrorr hydrogen; target located at edge of ring current distribution. . . . .

4-26 Characteristic signals from three-channel. X-ray deteotor and axial magnetic probes for an electron ring confined in a backfill of 410 mTorr hydrogen and 6 mTorr argon; target located at edge of ring current distribution. . . . . . . . . . . . . . 
Page

4-27 Average electron energy as a function of time for an electron ring confined in a backfill of 450 mTorr hydrogen; target located at axial center of ring current distribution. . 252

4-28 Average electron energy as a function of time for an electron ring confined in a backfill of 450 mTorr hydrogen; target located at edge of ring current distribution. . . . . 254

4-29 Average electron energy as a function of time for an electron ring confined in a backfill of 750 mTorr hydrogen; target located at edge of ring current distribution. . . . . . . 256

4-30 Average electron energy as a function of time for an electron ring confined in a backfill of 410 mTorr hydrogen and 6 mTorr argon; target located at edge of ring current distribution • 258

4-31 Geometric model of electron ring used in loss rate analysis... . . . . . . . 269

4-32 Betatron energy gain per electron as a function of time for various ring geometries and different axlal magnetic field decay rates. . . . 272

4-33 Collimation geometry used for calculation of Pilot B crystal detector efficiency. . . . 293 
4-34 X-ray detection efficiency for a rightcylindrical Pilot B crystal $10.1 \mathrm{~cm}$ in diameter and $10.1 \mathrm{~cm}$ long..........

4-35 Families of curves depicting Pilot B detector signal ratios as a function of primary electron energy parameterized to the various thicknesses of absorber used in the experiment. . . . . . . . .

4-36 Primary electron energy as predicted by various $\mathrm{Pilot} B$ detector signal ratios having different absorber combinations for a monodirectional and diffuse beam ......

\section{CHAPTER 5}

5-1 Schematic diagram of RECE-Berta with arcjet and associated field-grading coils attached.

5-2a One-half-scale section view of the MPVarcjet used for the preionization studies. .

5-2b Side and frontal views of the arcjet; the scale is in inches.............

5-3 (TOp): Electrical schematic of sections of an $\mathrm{N}$-stage, discrete element transmission line; (Bottom) alectrical schematic of the pulse-line used to energize the MPD-arcjet . 
5-4 (TOp): Photograph of the arcjet pulselinè (Bottom): Upper oscillographs are the net pulse-line current into $20 \mathrm{~m} \Omega$ load; Lower oscillographs show pulse-line voltage characteristics at two points along the line (cf. Figure (5-3))...........

5-5 Photograph of arcjet and graded-field section attached to the downstream end of Berta. . . . . . . . . . . . . . . .

5-6 Axial magnetic field in RECE-Berta with various sets of graded-field coils engaged .

5-7 (Top) : Illustration of Langmuir probe construction details; (Bottom): Electrical schematic of 'Langmuir probe bias and current monitor circuit. . . . . . . . . . .

5-8 Configuration of Langmuir probe inside RECE-Berta for plasma profile measurements .

5-9 Oscillographs showing Langmuir probe currents at various radial positions in the arcjet plasma; top trace is arcjet current profile; probe is located at the axial minimum of the magnetic mirror well (plasma density in $\mathrm{cm}^{-3}$ ). 
5-10 Arcjet plasma radial density profiles parameterized to time after the initialization of arcjet current; probe axial location is $130 \mathrm{~cm}$ from downstream end of Berta. . . . . . . . . . . 348

5-11 Arcjet plasma radial density profiles parameterized to time after the initialization of arcjet current; probe axial location is $177 \mathrm{~cm}$ from downstream end of Berta (about $6 \mathrm{~cm}$ away from the axial position of the end of the injector snout). - 350 5-12 Arcjet plasma density at the minimum of the magnetic well as a function of time after the initialization of arcjet current; probe radial position is at $\pm 10 \mathrm{~cm} \cdot \ldots 353$ 5-13 ('IOp) : $B_{\theta}$ field from the arcjet plasma. currents; (Bottom): Arcjet axial plasma current distribution. ...'. . . . . 359

5-14 Timing sequence used in the plasma-trapping experiments ............... 363

5-15 "Stalled". and "Trapped" modes of electron ring injection into a background plasma . . 366 
Page

5-16 Axial diamagnetic profiles characteristic of beam injection into a plasma density $n_{p}-1.3 \times 10^{12} \mathrm{~cm}^{-3}$........... 370

5-17 Axial diamagnetic profiles characteristic of beam injection into a plasma density $n_{p}-1 \times 10^{12} \mathrm{~cm}^{-3}$........... 372

5-18 Axial diamagnetic profiles characteristic of beam injection into a plasma density $\mathrm{n}_{\mathrm{p}}-5 \times 10^{11} \mathrm{~cm}^{-3}$............ 374

5-19 Axial diamagnetic profiles characteristic of beam injection into a plasma density $n_{p}-8 \times 10^{10} \mathrm{~cm}^{-3}$............ 376

5-20 Axial diamagnetic profiles characteristic of beam injection into a neutral background density $\mathrm{n}_{0} \sim 2 \times 10^{14} \mathrm{~cm}^{-3}$........ 378

5-21 Direct comparison of magnetic piekup probe signals with the $\mathrm{X}$-ray detector responses for injected currents at various axial positions in Berta. . . . . . . . . . . . .

5-22 High-energy circulating electron current axial profiles characteristic of beam injection into a plasma density $n_{p} \sim 1.3 \times 10^{12}$ $\mathrm{cm}^{-3}$................... 
5-23 High energy circulating electron current axial profiles characteristic of beam injection into a plasma density $n_{p}-1 \times 10^{12}$ $\mathrm{cm}^{-3}$. The "error bars" show shot-to-shot variations in the data. . . . . . . .

5-24 High-energy circulating electron current axial profiles characteristic of beam injection into a plasma density $n_{p} \sim 5 \times 10^{11}$ $\mathrm{cm}^{-3}$. The "error bars" show shot-to-shot variations in the data.......... 391

5-25 High-energy circulating electron current axial profiles characteristic of beam injection into a plasma density $n_{p}-8 \times 10^{10}$ $\mathrm{cm}^{-3}$...................

5-26 High-energy circulating electron current axial profiles characteristic of beam injection into a neutral background density $\mathrm{n}_{0}-2 \times 10^{14} \mathrm{~cm}^{-3}$........... 395

5-27 Electron ring trapping in a preionized medium without a quadrupole field . . . . 400 5-28 Long-term electron ring confinement in a preionized medium without a quadrupole field. 404 
5-29 The beam-plasma model of Chu and Rostoker. . 408

5-30 Illustration of the emergence of the beam diamagnetism behind the beam head. . . . .

5-31 Percentage of beam electron energy loss per centimeter $(" f ")$ as a function of beam axial velocity, $B_{z}=v_{z} / c . . . . \cdot . \cdot . \cdot 424$

\section{CHAPTER 6}

6-1 The principle features of cusp-injection . . 442

6-2 Transverse view (looking radially outward at the cusp field-piece) of the charged particle trajectories under the influence of the cusped field.............

6-3 Sketch of the magnetic fields present as an intense, hollow electron beam transits a cusped magnetic field. . . . . . . . . 449

6-4 Section view of the slotted cusp field-piece 4453

6-5 Schematic diagram of the cusp-injection apparatus. . . . . . . . . . . . 455

6-6 Axial magnetic field profile in the cuspinjection apparatus, . . . . . . . . . 460

6-7 The annular, carbon cathode used to create the hollow electron beam and one of the titanium anode foils; note the circular damage pattern on the anode foil. ......... 465 
Page

6-8 Transient neutral density profiles for hydrogen puffed into the cusp-injection apparatus : • • • • • • • • • • • •

6-9 Characteristic diode, pulsed-field current, diamagnetic probe, and $\mathrm{x}$-ray monitor signals for an electron ring confined in 80 mTorr hydrogen in the cusp-injection device. . .

6-10 Axial diamagnetic field profile of an electron ring confined in 80 mTorr hydrogen in the cusp-injection device . . . . . . . 478

6-11 Electron ring axial diamagnetic field decay rate as a function of backfill pressure

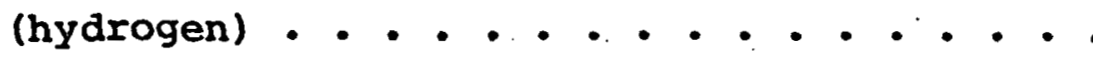

6-12 Axial diamagnetic field signals from plasmatrapped electron ring confined in the cuspinjection device . . . . . . . . . . .

6-13 Axial diamagnetic field profile of an electron ring confined in a plasma density $n_{p}-10^{14} \mathrm{~cm}^{-3}$ in the cusp-injection device. 
Page

\section{APPENDICES}

I-1 Main features of typical partial-finge shift data. . . . . . . . . . . . . .

I-2 (TOp): Configuration of interferometer in performing density measurements on the cuspinjection device; (Bottom): Photograph of the general interferometer layout on the cusp device (the railroad train is shown for scale). . . . . . . . . . .

I-3 Radial plasma density profile of the plasma column injected into the cusp tank. . . . 505

I-4 Plasma density in the cusp tank as a function of time (based on the radial density profile of Figure $(I-3))$. . . . . . . . . . .

II-1. Photon Mass Attenuation Coefficient for NaI (after Reference 87). . . . . . . . . 511

II-2 Photon Mass Attenuation Coefficient for Sn (after Reference 67). . . . . . . . . 513

II-3 Photon Mass Attenuation Coefficient for $\mathrm{Pb}$ (after Reference 159). . . . . . . . . 515

II-4 Photon Mass Attenuation coefficient for $\mathrm{Cu}$ (after Reference 67) . . . . . . . . . . 
III-1 Absorber and collimation geometry used for calculation of NaI(TI) crystal detector efficiency and absorber transmission coefficients. . . . . . . . . . . .

III-2 Schematic illustration of the details of the materials and surface designations used in the absorber transmission coefficient calculations. . . . . . . . . . .

III-3 Schematic illustration of the details of the materials and surface designations used in the detector efficiency calculations . . 530

III-4 The "weighted-slab" approximation to the cylindrical geometry of the wire target . . . 537 III-5a Comparison between the $2 \pi$-integrated Bremsstrahlung spectra from a $2.54 \times 10^{-3} \mathrm{~cm}$ thick tungsten slab and a $2.54 \times 10^{-3} \mathrm{~cm}$ diameter tungsten wire (weighted slab technique) . . . . . . . . . . . .

III-5b Comparison between the $2 \pi$-integrated

Bremsstrahlung spectra from a $2.54 \times 10^{-3} \mathrm{~cm}$ diameter tungsten wire (weighted slab technique) and a wire target of the same diameter incorporating the true cylindrical wire cross-section into the code computations ................. 
Page

III-6 Bremsstrahlung code histographs for spectra from $2.54 \times 10^{-3} \mathrm{~cm}$ diameter tungsten wire target (weighted slab technique). . . . . . 580

III-7 Differential Compton cross-section as a function of angle, $10 \mathrm{keV}<\mathrm{E}_{\gamma}<500 \mathrm{keV} \cdot .-583$

III-8 Total compton. cross-section as a function of photon energy. . . . . . . . . . . . 585

III-9 Gamma ray scintillation spectrometry for one of the NaI(TI) detectors used in the ring electron energy measurement. . . . . . 591 III-10 Peak-to-Total Ratio for $4.5 \mathrm{~cm} \times 5.1 \mathrm{~cm}$ right-cylindrical NaI(TI) scintillation crystal . . . . . . . . . . . . .

III-11 NaI(TI) crystal detection efficiency by simple calculation and by Monte Carlo in the $\mathrm{X}$-ray energy range $100 \mathrm{keV}<\mathrm{E}_{\gamma}<1000$

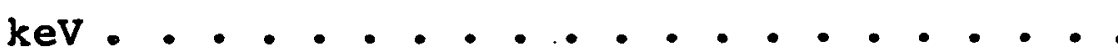

v-1 Characteristic arcjet current and voltage oscilloscope waveforms. . . . . . . . .

VI-1 (TOp): WL7676 fast ion gauge tube mounted on carriage and attached to umbilical; (Bottom): Gauge tube bias voltage supply and ion/electron current monitor. . . . . . 
Page

VI-2 Electrical schematic of circuits used for biasing the gauge tube and monitoring the ion/electron currents flowing in the tube. . 617

VI-3 Static gasfill calibration of the ion gauge tube in hydrogen . . . . . . . . . . . 620

VI-4 Hydrogen neutral pressure as a function of time at the minimum of the magnetic well in the cusp-injection device; inset in the upper left-hand corner of the plot are a pair of typical collected ion- and electroncurrent oscilloscope traces for data collected at this particular axial location of the gauge tube (the interruption of the trace brightness is a timing fiducial mark via z-axis modulation). 
CHAPTER 1

INTRODUCTION

$-1-$ 


\section{CHAPTER 1}

\section{INTRODUCTION}

\subsection{The Astron}

Since its inception in the $1950^{\prime} \mathrm{s}$, research in controlled thermonuclear reactions has, of necessity, given birth to a fairly sizeable clutch of diverse schemes whose ultimate, common goal has been the confinement of high-temperature plasma at densities and for time intervals sufficient to initiate nuclear fusion reactions. The importance of stabilizing the collective magnetohydrodynamic "fluid" instabilities, to which such plasmas could be susceptible in almost any number of subtle ways, was recognized early in the game and was a point of central emphasis in the theoretical design behind most of the confinement devices. Consider, for example, the case of magnetic confinement. The open-field "mirror machines" displayed magnetic single-particle confinement in full agreement with adiabatic theory, with particle containment between the mirrors having been achieved for $10^{9}$ transits of the mirror trap. ${ }^{1}$ Unfortunately, proceeding to fill such a mirror with a bulk plasma also brought on the MHD "flute", or "interchange" instability. Production of 
the catastrophic flutes in such a magnetic field geometry was theoretically predicted in the work of Rosenbluth and Longmire. ${ }^{2}$ However, these authors also obtained a sufficient condition for the absolute stability for such a magnetically-confined plasma, i.e. ., that $\delta \int d l / B<0$ be satisfied at all points inside the plasma, where the integration is carried out along a line of magnetic flux inside the plasma volume, and the " $\delta "$ implies the change in the integral between adjacent "flux tubes" in the plasma. This sufficiency criterium for stabilization of the interchange mode is actually a special case of a more general criterium based on an energy-principle analysis of MHD plasma stability which says that a plasma is absolutely stable if the confining magnetic field increases in all directions away from the plasma surface. ${ }^{3}$

In the case of mirror machines, the interchange mode was experimentally stabilized by the application of multipole magnetic fields ${ }^{4}$ which satisfied the "Minimum-B" criterium by causing the magnetic field pressure, $\mathrm{B}^{2} / 8 \pi$, to increase in all directions from the center of the plasma. However, such stabilization does not eliminate particle losses by collisional scattering into the "loss-cone", a type of velocity- 
space diffusion inherent to the field geometry of the magnetic mirror. 5

A class of "internal ring" confinement devices was developed which sought to achieve the "Average Minimum-B" magnetic field configuration for plasma containment through the use of current-carrying structures internal to the plasma. The inherent difficulty with. this multipole concept for fusion reactor applications is the presence of these conductors inside the fusion plasma, where they would not only act as a "sink" for the plasma, but would also be subjected to a withering flux of $x$-radiation and heavy particle bombardment. In 1958, Nicholas Christofilos proposed that these difficulties could be avoided by replacing the conductors with a circulating ring of charged particles. ${ }^{6}$ If one were to confine this circulating ring of charge within a mirror machine, and enough current could be made to circulate within the ring, then the magnetic field on the axis of the device could be made to reverse direction, as shown in Figure (1-1). Consequently, within the ring current distribution, a region of closed, nested magnetic field lines is created in which the magnetic field increases in all directions from the ring center. This concept was subsequently 
FIGURE 1-1

Conceptual view of an electron ring confined in a magnetic mirror well. 


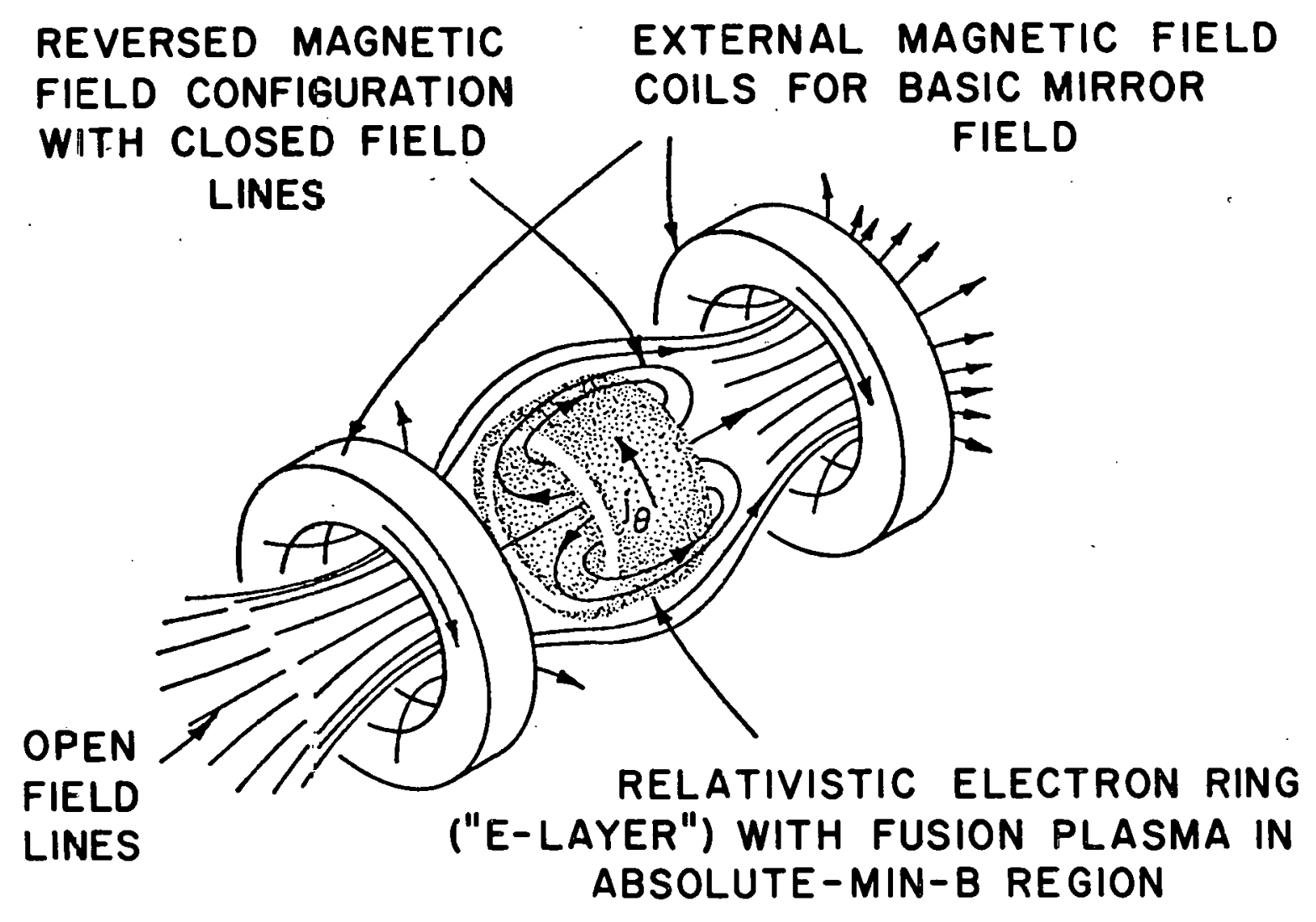


embodied in the experimental effort at the Lawrence Radiation Laboratory in Livermore, California, known as the Astron.

The advantages of using "E-layers" (or "Relativistic Electron Coils"7), as they were called by Christofilos, within the plasma are really twofold: (1) the creation of the above-mentioned stable magnetic confinement structure for the plasma, and (2) a direct mechanism by which the plasma so confined could be heated via collisions between the circulating relativistic electrons and the plasma particles. Unfortunately, the synchrotron radiation losses from the circulating electrons at energies $\geq 40-50 \mathrm{MeV}$ would pose a prohibitive restriction on using such electron rings in an operating fusion reactor. Instead, circulating currents of protons would probably be employed for this purpose. However, the technology to produce intense beams of high energy electrons was far less costly and much more manageable than that for heavy ion beams. Hence, electron beams were (and are) used in this work as a first step in exploring the physics of intense current rings, with the intent of making the transition to intense proton beams when they become available.

The accelerator used to inject electrons into the 
Astron experiment could produce $600 \mathrm{~A}$ bursts of electrons at energies of up to $6 \mathrm{MeV}$ with a pulse width of 20.0ns. These particles were injected at one end of the long mirror well in a direction almost perpendicular $\left(\sim 5^{\circ}\right)$ to the axis of the device. The circulating electrons would then drift down the axis to the bottom of the "magnetic well", where they were confined as a semirigid current sheath ("E-layer"). However, the highvacuum trapping of typical pulses of electrons from this accelerator was found to generate E-layers whose diamagnetic strength (called " $\zeta$ " and defined in cornell parlance as being the ratio of the maximum ring selffield on axis to the base solenoidal magnetic field, $\underline{i} . \underline{e} .$, $\zeta=\mathrm{B}_{\mathrm{MAX}} / \mathrm{B}_{0}$ ) was about $4 \%$ of that needed for field reversal; single-pulse trapping in high pressures of hydrogen (200 mTorr). gave rise to electron rings with self-fields of about $40 \%$ of the base magnetic field. 8 Thus, the success of the Astron program was predicated on being able to "stack" several consecutively-injected electron coils together, thereby building up the circulating current until field-reversal could be achieved. Unfortunately, pulse-stacking of E-layers was not successful: stacking injected electron beam pulses consecutively was found (i) to saturate the E-layer current density at field-reversals of about $18 \% ; 9$ and 
(ii) to cause the E-layer to lengthen as more pulses were added. Furthermore, large particle losses were observed during the injection of pulses following the initial electron pulse before these particles could reach the mirror trap. ${ }^{10}$ Attempts to alleviate these difficulties by stacking layers in high vacuum (giving better axial compression via slower charge neutralization) were plagued by particle losses during compression, and did not give encouraging results. ${ }^{11}$. The Livermore Astron program was terminated in 1973.

1.2 The Corne1l Relativistic Electron Coil Program With the advent of pulsed, high-current relativistic electron beam technology, a new approach to particle injection into Astron-like magnetic field geometries was explored at Cornell University in 1968. In this scheme, single pulses of electrons which contained almost two order of magnitude more charge than was necessary for E-layer field-reversal were injected laterally into a magnetic mirror geometry. As reported by Andrews, et al. 12 the work was successful, with the creation of electron rings with self-fields greater than the base axial mirror field $(\zeta>1)$ at beam energies of $340 \mathrm{keV}$ and currents of about 30-60 kA. The net electron ring lifetimes in this work were limited to a couple 
of hundreds of nanoseconds because of the collision of the ring electrons with the injector snout (which protruded into the trapped ring itself). Nonetheless, the difficulties of pulse-stacking had been avoided with this technique and, since approximately fifty times the amount of charge needed to obtain field-reversed rings was actually injected into the device, trapping efficiency ceased to be a matter of such delicate experimental concern.

A second-generation ring containment device was constructed at Cornell which was considerably larger than the first machine. The main impoovement realized with this second device, called "Berta", was that longer ring. lifetimes could be expected by having removed the injector sriout back into one of the magnetic mirrors. This machine was successful soon after. it went "on-line", producing field-reversing electron rings with lifetimes of $20 \mathrm{\mu sec}$ in 500 mTorr hydrogen. ${ }^{13}$ For reference, the relative scale between the cornell and Livermore experiments is shown in Figure (1-2).

A set of sample oscilloscope traces illustrating the axial diamagnetic behaviour of a confined electron ring as a function of time, as well as a time-integrated photograph of one of the rings confined in RECE-Berta 
$-11-$

FIGURE 1-2

Scale comparison between the Astron and RECE-Berta. 





(taken from the injector end of the device) are shown in Figure (1-3). Characteristically, the electron rings display three common, salient features: (1) a very rapid (less than $0.5 \mu \mathrm{sec}$ ) initial buildup to some maximum diamagnetic strength, followed by (2) a fairly linear decay of ring diamagnetism until (3) there is a rapid loss of signal and circulating current at ring diamagnetic strengths of about $\zeta \sim 10-20 \%$. In consequence of these features (which are, with the exception of the field-reversal strength, virtually identical to the E-layer characteristics of the Livermore Astron), the first areas of investigation were an understanding of the linear decay rate and the rapid "dump" which terminates the ring's lifetime. The results of these two sets of investigations were encouraging from a "scaling" point of view: Experiments with background gasfills of varying atomic number confirmed that the linear decay rates observed were consistent with the predictions of classical scattering of the fast electrons with the constituent backfill gas. 14 (2) The "dump" was found to be analogous to the "precessional instability" found in the Livermore experiments, with the onset of the instability having been augmented, in the cornell experiments., 


\section{$-14-$}

\section{FIGURE $1-3$}

(Top) Time-integrated photograph of electron ring trapped in RECE-Berta (photo taken from upstream end of Berta);

(Bottom) Typical electron ring diamagnetic signals showing trapping, linear decay, and "dump" features. 


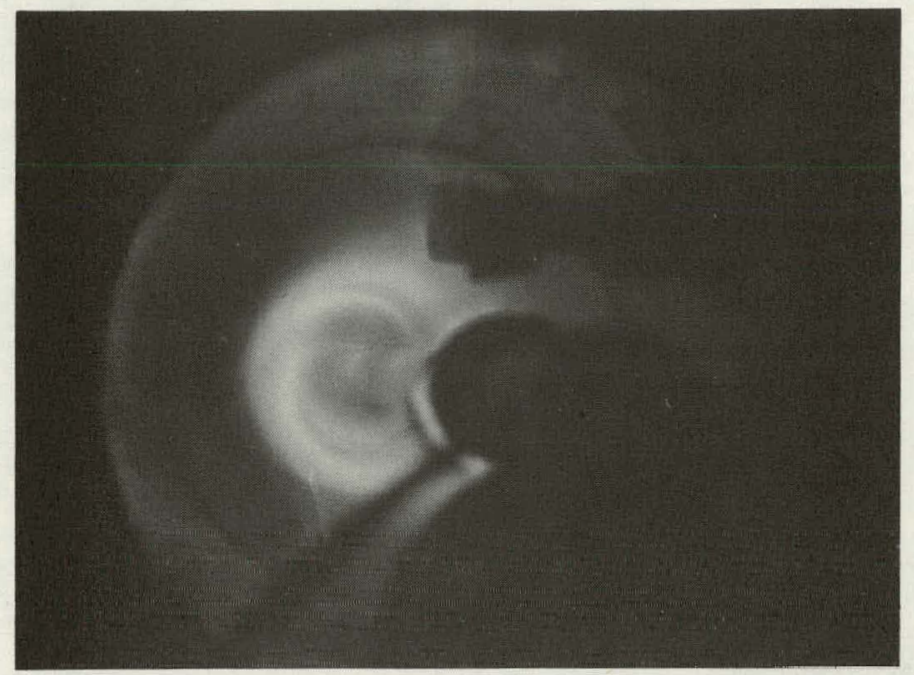

ELECTRON RING CONFINED IN RECE-BERTA

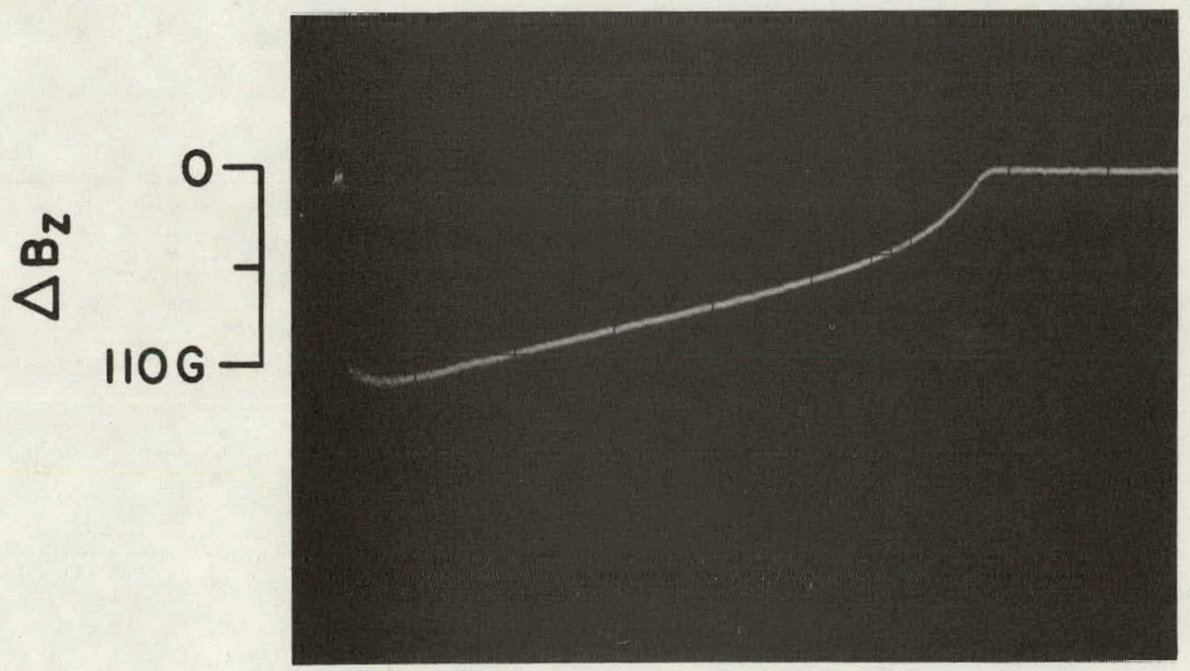

RING AXIAL DIAMAGNETISM

$2 \mu s /$ div $\rightarrow$ 
by the presence of field gradients in the background magnetic field. It was found 15,16 that the dump could be stabilized via the application of either a toroidal field (making the magnetic field structure within the machine similar to that of the "spherator"), or a Ioffe-like quadrupole field, a significant achievement from the fusion reactor point of view since it would permit (ion) ring stabilization without a central conductor running through the middle of the device. Studies were also performed on the sensitivity of the rings to externally-applied magnetic field gradients in order to explore permissible tolerances in magnetic field design. Meger ${ }^{17}$ found that transverse field differences of up to $0.8 \%$ were permissible before the rings were driven unstable. Also, as groundwork for electron ring compression studies, Luckhardt ${ }^{18}$ has shown that it is possible to translate the electron rings axially to a new location through the application of fast-rising axial magnetic field gradients.

\subsection{Objectives of the Research}

It is the purpose of this report to describe experimental work that has been concerned with the trapping and energy loss mechanisms of intense relativistic electron rings. The work represents four distinct areas 
of experimental investigation:

\section{(1) Ring Trapping in Hydrogen Gas.}

There are significant differences between the trapping processes at work for the electron rings confined in the cornell experiments and the E-layers generated in, the Astron. The Livermore Astron utilized a concentric network of resistive wires inside the "drift region" to dissipate some of the injected beam's axial energy so that the ring would not reflect off of the downstream mirror and return to collide with the injector. Thus, the E-layers were formed as an entity near the point of injection and drifted down into the mirror well region with an initial axial speed of about $0.1{ }^{19}$ After some axial oscillations back and forth at the bottom of the well, the E-layer settled down in the minimum of the well, where it remained. This behaviour is shown in Figure (1-4).

In the Cornell experiments, no such resistive structure was mounted inside the containment vessel, with reliance upon (as yet undetermined) interactions between the injected beam and the high gas backfill pressures for the dissipation (or scattering) of the beam's axial energy. The trapping efficiency in the cornell experiments is typically low ( $2 \%)$, to 


\section{FIGURE $1-4$}

Position of the peak current density of an E-layer confined in the Astron as a function of time. The curve approximates the position of the center of the circulating charge distribution. (Beam energy = $5.8 \mathrm{MeV}$, Beam current $=200 \mathrm{~A}$, Mirror depth $=28$, Hydrogen pressure $=17$ mTorr $)$. (From Reference 19). 


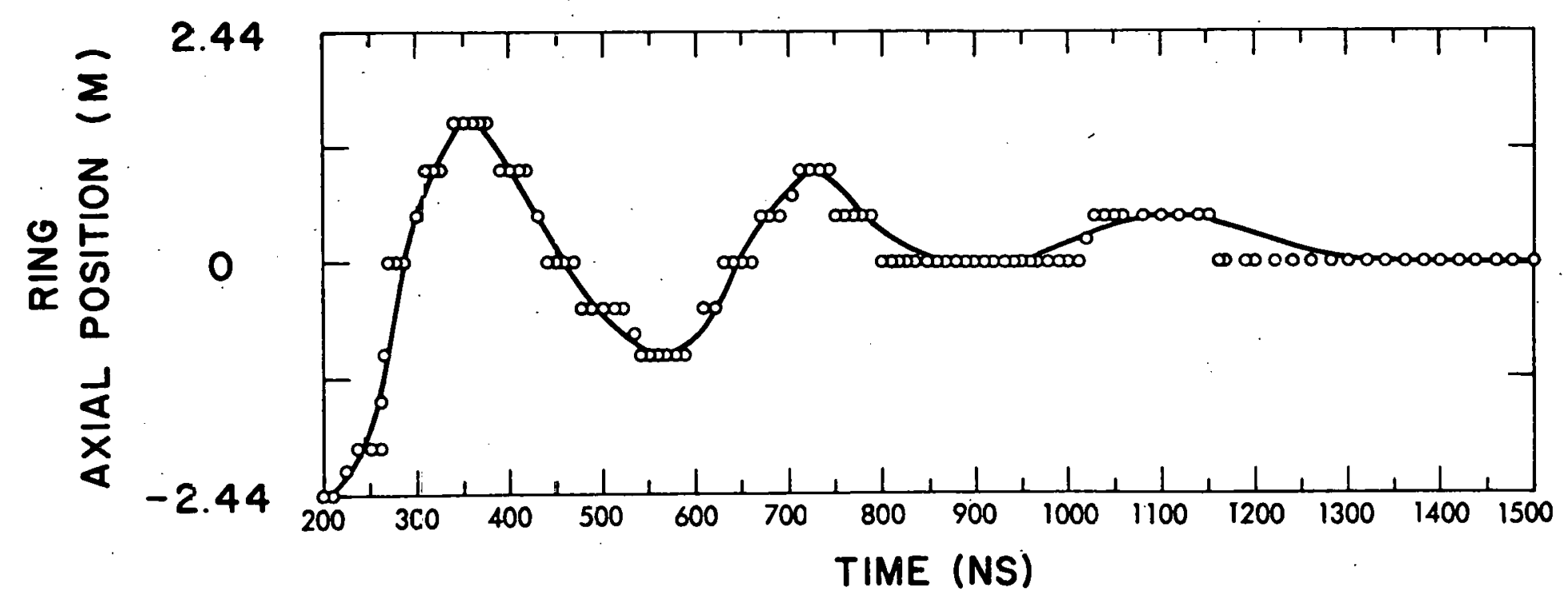


be contrasted with the trapping efficiency in the Livermore experiments, which reached about $60 \% .20$ Hence, it was apparent that some experimental work should be undertaken to study the beam trapping processes taking place under the conditions of the cornell injection scheme.

The experimental work described here did not deal with the highly complex loss mechanisms at work as the beam is injected but, rather, with the dynamic behavior of the circulating relativistic electron currents found to be present in the machine between $100 \mathrm{~ns}$ and $800-1000 \mathrm{~ns}$ following injection of the electron beam into the mirror trap.

Under all experimental conditions, in contrast to the Livermore results, it was found that the mirror well was filled initially to its full axial length with a quite uniform, circulating cylinder of charge which then "relaxed" towards an equilibrium distribution revealing the steady-state diamagnetic profile of the confined ring. The rate at which this relaxation proceeded was found to vary slightly with backfill pressure and was always completed within $500 \mathrm{~ns}$ after injection.

This phenomenology was analyzed in the context of the tensor conductivity of the beam-created background plasma. The experimental results were found to be consistent with the magnctic penetration times calculated 
for a cylinder of circulating plasma currents, with the beam-induced angular counter currents having both Hall and Pedersen contributions, in varying proportions depending on the background gas density (i..e.., electronneutral collision frequency). As these induced currents abated, the circulating high-energy electrons could correspondingly assume an axially-focussed, steady-state equilibrium current distribution.

\section{(2) Average Ring Electron Energy Measurement.}

As mentioned earlier, investigation of the linear ring self-field decay rates showed that the loss of circulating current during the lifetime of the electron rings was consistent with the classical scattering of the high-energy electrons with the particles of the background gases in which the rings were confined.. Although these results also implied that the fast electron energy losses were classical as well, a direct experimental confirmation of that possibility was required. In addition, such a direct energy measurement would also indicate whether the rings were being subjected to deleterious instabilities during their residence in the mirror trap.

To this end, a diagnostic technique was developed in which the primary electron energy could be 
deduced via multi-foil absorption spectroscopy of the Bremsstrahlung generated as the circulating high energy electrons impinged upon a small tungsten target. Electron and photon transport codes made available for this work by the Los Alamos Scientific Laboratory (and which were also operated at LASL) permitted computation of the necessary Bremsitrahlung spectra, as well as spectral analysis of the $\mathrm{X}$-radiation as it traversed the absorbers and collimation geometry used in the experimental configuration. A simple, classical analysis of a ring of current modelled to obey the experimentally-observed dynamic characteristics of the confined electron rings revealed that the experimentally-measured energy loss of the circulating ring electrons could be explained by classical energy losses of the fast particles in the gas coupled to a betatron-like acceleration of the particles remaining in the ring (brought on by the rapidlychanging magnetic flux through the center of the ring).

\section{(3) Electron Beam Trapping and Confinement in} Hydrogen Plasma.

Having established that fast electron scattering with the background gas. limited the lifetimes of the strong electron rings contained in the Cornell experiments, the next question was whether the lifetimes 
of these rings could be enhanced by some classical means. This question was pursued with the $500 \mathrm{keV}$ injection facility by replacing the background gasfills with a preionized medium of hydrogen plasma having a density which was 3-4 orders of magnitude lower than the usual hydrogen backfills used in gas trapping. With this approach, it was found that the electron ring lifetimes were extended by a factor of 15-20 to approximately $400 \mu \mathrm{sec}$. However, the trapping efficiency was substantially lower in the background plasma, with confined rings of $\zeta \leqslant 25 \%$. By far the most singular aspect of the preionization experiments turned out to be the beam trapping process itself, which bore no resemblance to the gas trapping in that the beam was observed to "stall" near the point of injection. Whether the circulating ring current then proceeded down the axis of the machine to the bottom of the magnetic well (behaviour reminiscent of the Livermore resistive trapping process) appeared to depend solely upon the density of the plasma into which the beam was injected. One possible--but by no means definitive--explanation for this train of events is suggèsted in the theory developed by Chu and Rostoker, ${ }^{21}$ in which the interaction of a circulating annular beam travelling through a background plasma is examined. In this model, the decay of 
the beam-induced counter currents. in the plasma was predicted to produce strong axial forces on the beam which could abate its axial progress over distances which were consistent with those observed in these experiments.

\section{(4) Cusp-Injection.}

The final topic considered in this work dealt with an alternate method of injecting a rotating beam down the axis of a mirror machine. In an extension of the experiments of Kribel, et al.,$^{22}$ an annular electron beam was passed down the axis of a cusped-magnetic field located coaxially to, and at one end of, a magnetic mirror well. The purpose of this preliminary work was to determine whether the trapping efficiency was enhanced through such an injection scheme, and whether long-lived electron rings could be formed by firing cusp-injected electron beams into puffed-gas backfills of hydrogen gas, as well as into a preionized medium. Electron rings with lifetimes of over $140 \mu \mathrm{sec}$ were achieved by injection of the beam into a dense $\left(n_{p} \sim 10^{14} \mathrm{~cm}^{-3}\right)$ plasma, which corresponds to about $2 \times 10^{5}$ gyro-orbits of the circulating electrons. No improvement over the trapping efficiency of the transverse injection technique was found, however. 
1.4 Publications and Presentations Related to this Work

\section{PUBLICATIONS:}

Bzura, J. J.; Fessenden, T. J.; Fleischmann, H. H.;

Phelps, D. A.; Smith, Jr., A. C. ; Woodall, D. M.,

Physical Review Letters 29, 256 (1972). "Trapping

of High-Current Relativistic Electron Beams in a

Magnetic Mirror Trap."

Phelps, D. A.; Smith, Jr., A. C.; Woodall, D. M.; Meger,

R. A.: Fleischmann, H. H., Physics of Fluids 17 ,

2226 (1974). "Observations of the Stable Equi-

librium and Classical Diffusion of Field Reversing

Relativistic Electron Coils."

Smith, Jr., A. C.; Swannack, C. E.; Fleischmann, H. H.;

Phelps, D. A., Nuclear Fusion 15, 547 (1975).

"Energy Losses from Strong Electron Rings Trapped

in RECE-Berta."

Swannack, C. E.; Smith, Jr., A. C.; Fleischmann, H. H.;

Schrandt, R. C.; Thompson, W. L., "Time-Resolved

Energy Measurements on Relativistic Electron Rings

Using Mass-Absorption Spectroscopy." To be submitted to the Review of Scientific Instruments. 


\section{PRESENTATIONS:}

*Fleischmann, H. H.; Bzura, J. J.; Fessenden, T. J.; Meger, R. A.; Phelps, D. A.; Smith, Jr., A. C.: Woodall, D. M., Bulletin of the American Physical Society 17, 998 (1972). "Generation of FieldReversing Electron Rings Using Astron ${ }^{-T y p e}$ Injection of High-Current Electron Beams."

*Phelps, D. A.; Smith, Jr., A. C.: Fleischmann, H. H., Bulletin of the American Physical Society 17, 999 (1972). "Shape and Collisional Decay of the strong Electron Layers Observed in RECE-Berta."

** Smith, Jr., A. C.; Swannack, C. E.; Phelps, D. A.; Fleischmann, H. H., Bulletin of the American Physical Society 18, 661 (1973). "Energy Changes of the Relativistic Electrons Trapped in RECEBerta."

*Fleischmann, H. H.; Swannack, C. E.; Phelps, D. A.; Meger, R. A.; Smith, Jr., A. Co; Woodall, D. M., Bulletin of the American Physical Society 18, 1283 (1973). "Generation and Long-Time Confinement of Strong Relativistic Electron Rings in RECE-Christa." 
"Swannack, C. E.; Smith, Jr., A. C.; Phelps, D. A.; Fleischmann, H. H., Bulletin of the American Physical Society 18, 1283 (1973). "Energy Analysis of the Relativistic Electron Rings Confined in RECE-Berta."

*Smith, Jr., A. C.; Shinsky, K.; Fleischmann, H. H.-, Bulletin of the American Physical Society 18 , 1283 (1973). "Trapping of Cusp-Injected Relativistic Electron Rings in Hydrogen Gas and Plasma." "Smith, Jr., A. C.; Fleischmann, H. H., Bulletin of the American Physical Society 19, 875 (1974). "Experiments on the Trapping Process in RECE-Berta." *Smith, Jr., A. C.; Fleischmann, H. H., Bulletin of the American Physical Society 20, 1290 (1975). "The Trapping Mechanism of Relativistic Electron Rings in the RECE-Berta Facility."

${ }^{\S}$ Smith, Jr., A. C.; Swannack, C. E.; Fleischmann, H. H.; Thompson, W. L.; Schrandt, R. C., Paper \#D-9. "Energy Measurements on Relativistic Electrons ( $E \leq 1 \mathrm{MeV}$ ) Using X-Ray Absorption Spectroscopy." *Smith, Jr., A. C.: Fleischmann, H. H., Bulletin of the American Physical Society 21, 692 (1976). "Trapping and Confinement of Intense Relativistic 
Electron Rings in a Background Hydrogen Plasma." * Abstracts of talks presented at meetings of the American Physical Society, Division of Plasma Physics.

$\star \star$ Abstracts of talks presented at spring Meeting of the American Physical Society.

${ }^{5}$ Abstract of talk presented at the Conference on Diagnostics of High Temperature Plasmas, January 7-9, 1976, Knoxville, Tennessee. 
CHAPTER 2

EXPERIMENTAL APPARATUS

$-29-$ 


\section{CHAPTER 2}

\section{EXPERIMENTAL APPARATUS}

\subsection{Introduction}

The apparatus used throughout most of the work described in Chapters 3-5 of this report is best described by subdivision into two discrete components: the electron beam generator, and the ring containment structure. Basically, the operation of the device consists of injecting a pulsed, relativistic electron beam (nominally 100 ns FWHM, $500 \mathrm{keV}, 15 \mathrm{kA}$ ) at an angle into one end of a magnetic mirror machine. The injected beam then spirals back and forth in the mirror fields, with approximately $1-28$ of the electrons eventually settling down to form an electron ring whose diamagnetic field strength can be as high as twice the value of the magnetic field at the bottom of the mirror well.

Detailed descriptions of both the beam generator 23,24 and the ring containment structure ${ }^{25}$ have been given elsewhere. For orientation, a brief sketch of this equipment is inclunted here.

\subsection{QWIBLE}

The electron beam generator is a tandem 10-stage 
Marx generator/folded-planar-Blumlein combination which has been electrically loaded into a high-vacuum, fieldemission diode. (The Blumlein has been dubbed QWIBLE, an acronym for Quick Water Insulated BlumLein.) The Marx generator acts as a moderately fast-risetime, highvoltage charging supply for the Blumlein pulse-forming network which is.actually responsible for the $100 \mathrm{~ns}-$ pulsewidth electron beams.

The characteristic impedance of the Blumlein is $25 \Omega$, with the diode impedance usually falling in the range of $30-33 \Omega$.

The electron beam characteristics which were monitored continuously throughout the experimental work were the diode current and voltage. A set of sample diode oscilloscope traces is shown in Figure (2-1). A timing fiducial is added for synchronism of the array of diagnostic oscilloscopes used in the experiments.

\section{$2.3 \quad$ RECE-Berta}

"RECE-Berta" is one of two electron ring production and containment devices used in the work described here. The other machine, a cusp-injected mirror machine, will be described in Chapter 6. "RECE" is an acronym for Relativistic Electron Coil Experiment; "Berta" has no particular significance. 
$-32-$

FIGURE 2-I

QWIBLE electron beam diode voltage and current waveforms. 


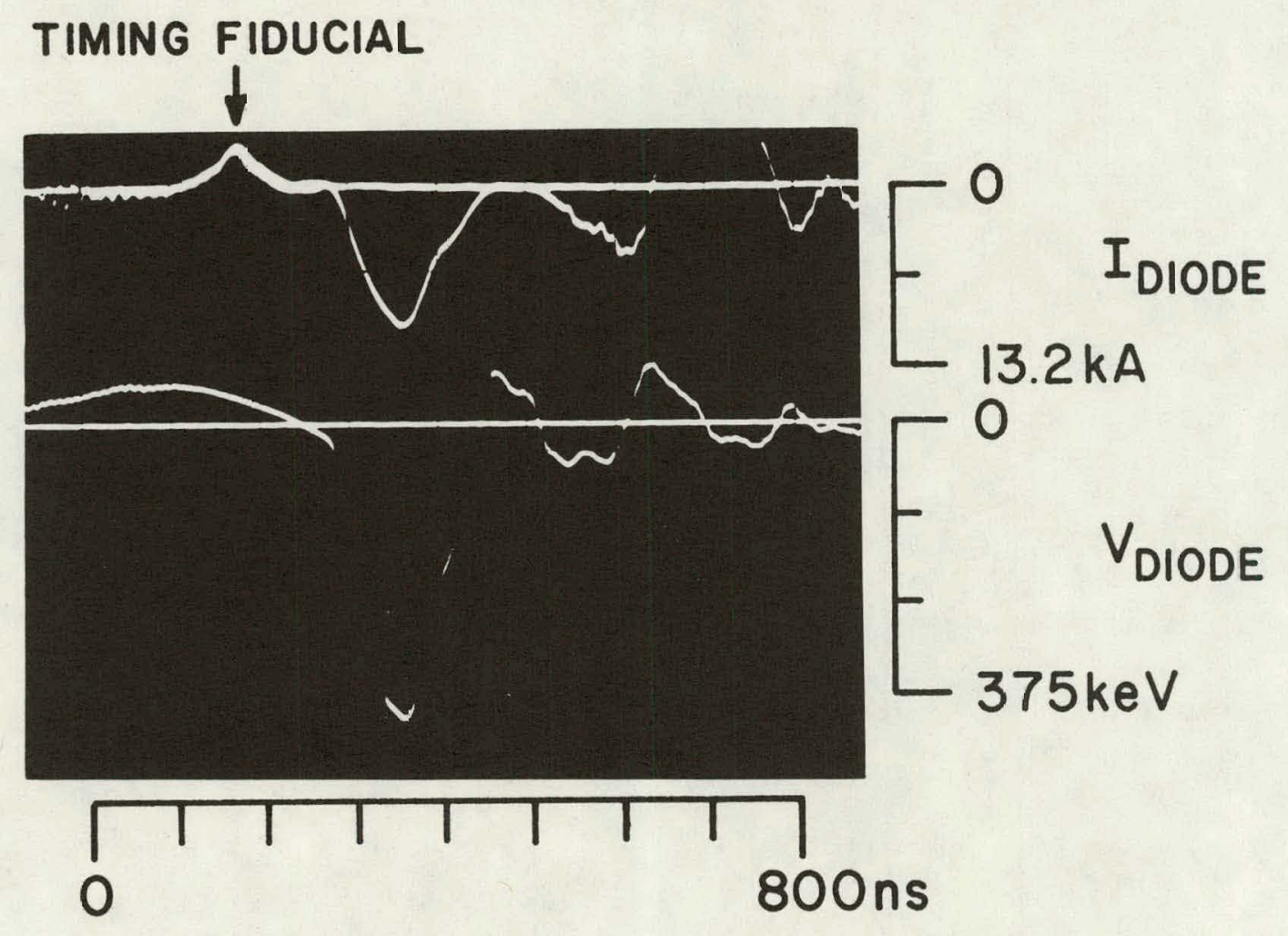


A sketch of the RECE-Berta facility is shown in Figure (2-2). Basically, the device consists of a $1.8 \mathrm{~m}$ long by $44 \mathrm{~cm}$ ID glass/brass vacuum vessel aligned coaxially with a set of solenoid magnetic field windings. The solenoid winding density is augmented at either end of the device by the addition of "mirror" fields which serve to trap and confine the injected electron beam. The interior walls of the glass vessel and plexiglass endplates were lined with Lexan (to protect the glass from the blast of high-energy electrons) and a set of copper meshes which provided a conducting, cylindrical boundary having a magnetic field penetration time measured to be $-900 \mu \mathrm{s}$. With the possible exception of those electron rings to be trapped in a preionized medium, this penetration time far exceeds the standard ring lifetimes anticipated in the device. Glass and plastic tubes housing magnetic probes, X-ray targets, etc., could be inserted axially into the machine through vacuum ports in the endplates.

The vacuum vessel was fitted with a $15 \mathrm{~cm}$ oil

diffusion pump to provide base pressures of $\sim 5 \times 10^{-5}$ Torr for the preionization studies. When required, backfill gas could be admitted into the vacuum chamber through a manifold of micrometer needle gas valves located on the downs tream endflange. 
$-35-$

FIGURE 2-2

Schematic illustration of RECE-Berta. 


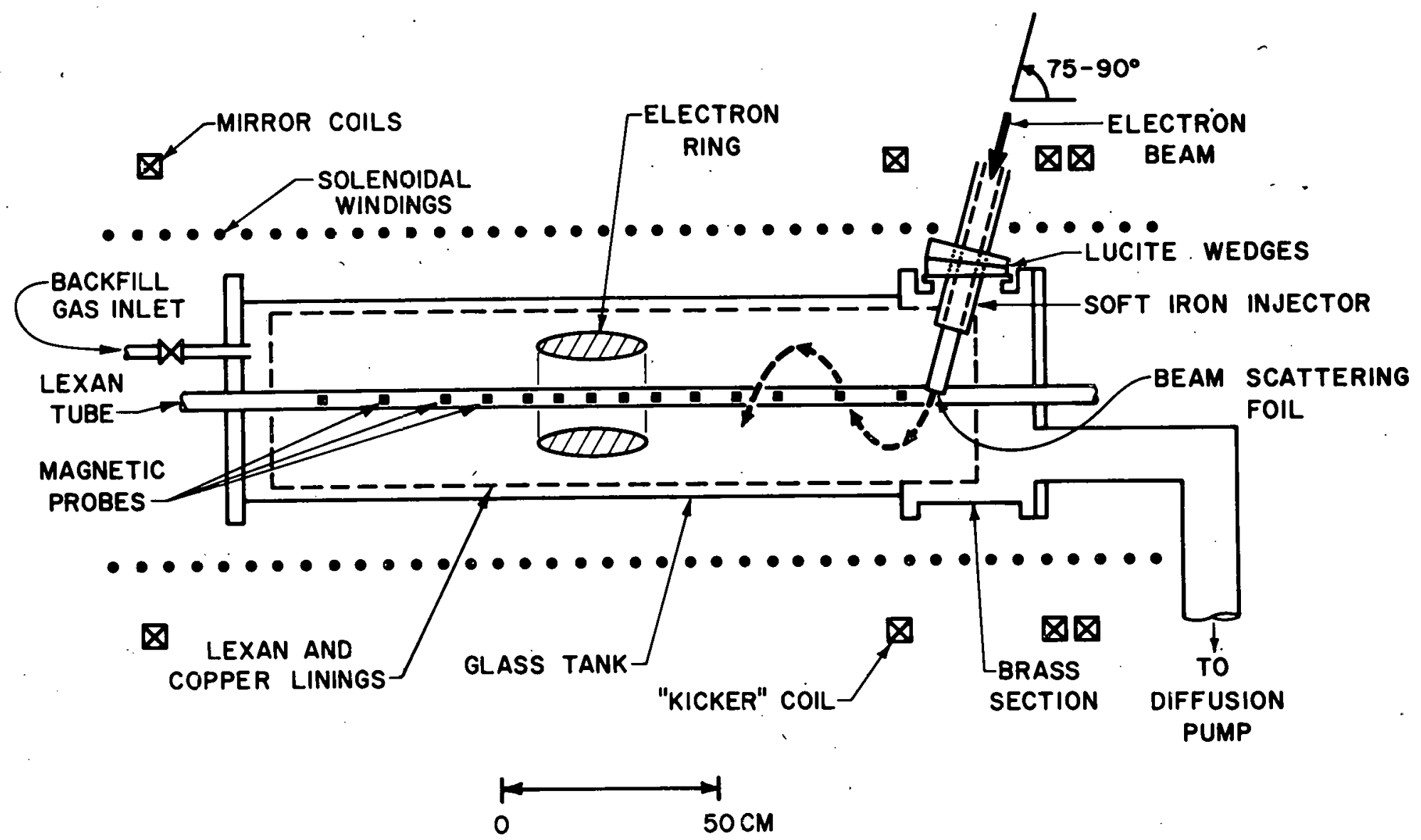


As shown schematically in Figure $(2-3)$, the field coils were pulsed from a $50 \mathrm{~kJ}$ crow-barred capacitor bank. A $450 \mathrm{mH}$ ballast coil was placed in series with the solenoid and mirror windings of Berta (inductance $-85 \mathrm{mH}$, resistance $\sim 8$ 8) so that small changes in the number of turns on the mirror windings to meet various experimental conditions would not affect the overall waveform of the current pulse (1/4-cycle risetime $14 \mathrm{~ms})$. Typically, the electron beam was fired at a time delay between 18 and $22 \mathrm{~ms}$ after the initialization of the current pulse to allow full field penetration of the brass section. A characteristic field current pulse is shown in Figure (2-3). The axial base magnetic field profile shown in the upper portion of Figure $(2-3)$ was found experimentally to be the most successful in terms of beam trapping in neutral gas. In addition, it was possible to impress a quadrupole (Ioffe) magnetic field onto the confinement volume. As will be discussed in Chapter 5, this additional field was found to be quite useful in the dumpstabilization ${ }^{26}$ of electron rings trapped in a background plasma, where geometry prohibited use of a toroidal magnetic field for this purpose. 27 Also, a perturbation coil was usually mounted on top of the device to counteract magnetic field gradients brought about by the steel substructure in the cart on which Berta was mounted. 
FIGURE 2-3

(Top) Typical axial magnetic field profile in RECE-Berta;

(Lower Left) Electrical schematic of the pulsed magnetic field circuit for RECE-Berta;

(Lower Right) Typical pulsed-field current waveform. 


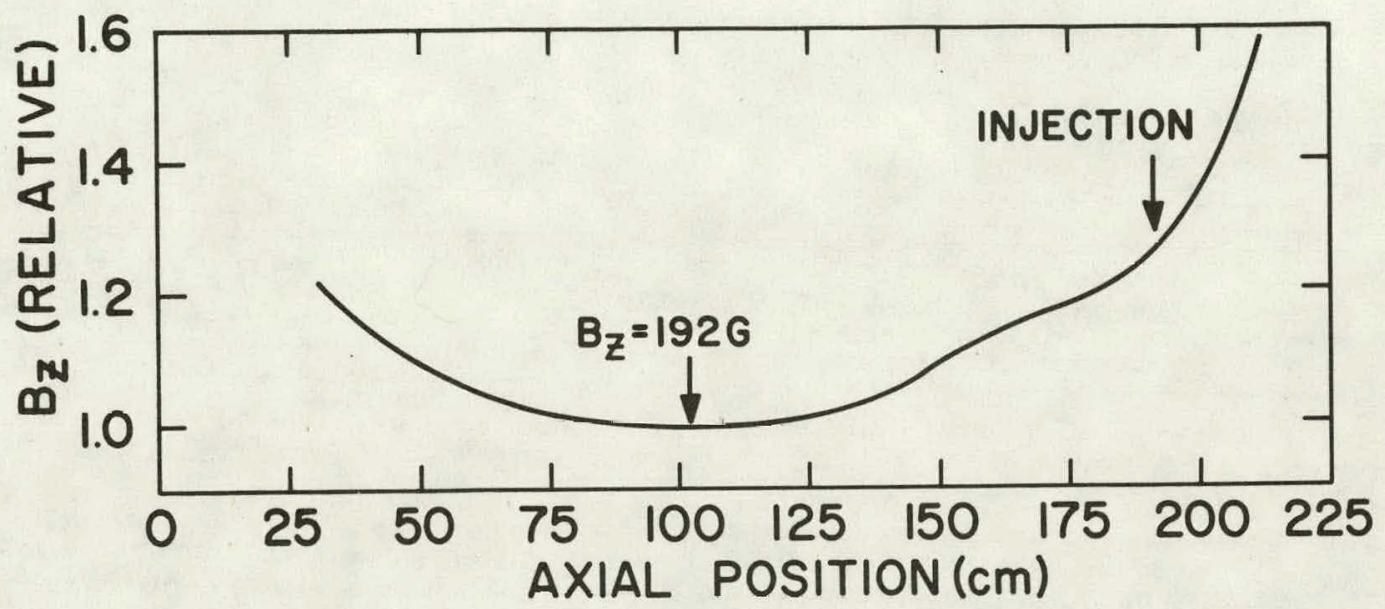

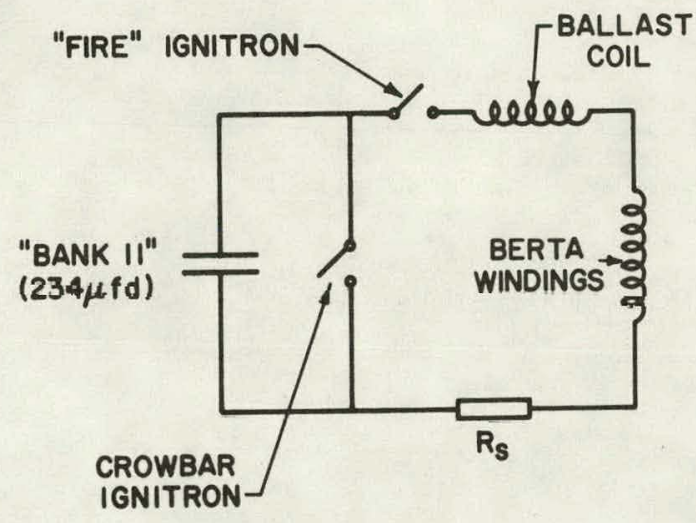

PULSED-FIELD CIRCUITRY

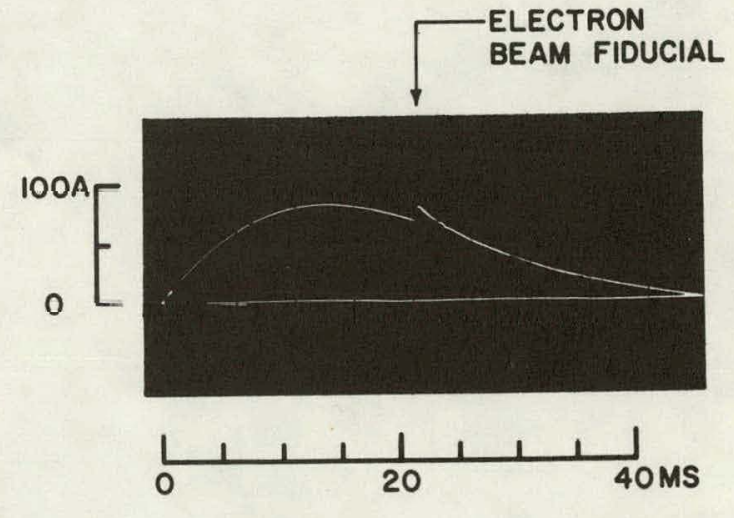

BANK CURRENT PULSE 
The electron beam is injected into this device at an angle of $5-15^{\circ}$ (from the perpendicular) from the end of a pressurized (1.5 Torr hydrogen), magneticallyshielded "drift tube" which is connected to QWIBLE's field-emission diode. A titanium foil at the end of the drift tube serves three purposes: (1) to isolate the main tank vacuum from the drift tube pressure; (2) to scatter the injected electrons through an angle of about $20^{\circ}, 28$ and (3) to suppress any low-energy electrons which might be produced in the diode from a second Blumlein "ringing" pulse.

A photograph of the RECE-Berta facility is shown in Figure $(2-4)$. 
Photograph of the RECE-Berta facility. Shown are the Marx-generator, QWIBLE, RECE-Berta, the cuspinjection device (CF. Chapter 6 ) in the foreground, and the $\mathrm{X}$-ray multi-channel absorption spectrograph to the left of Berta (cf. Chapter 4). 


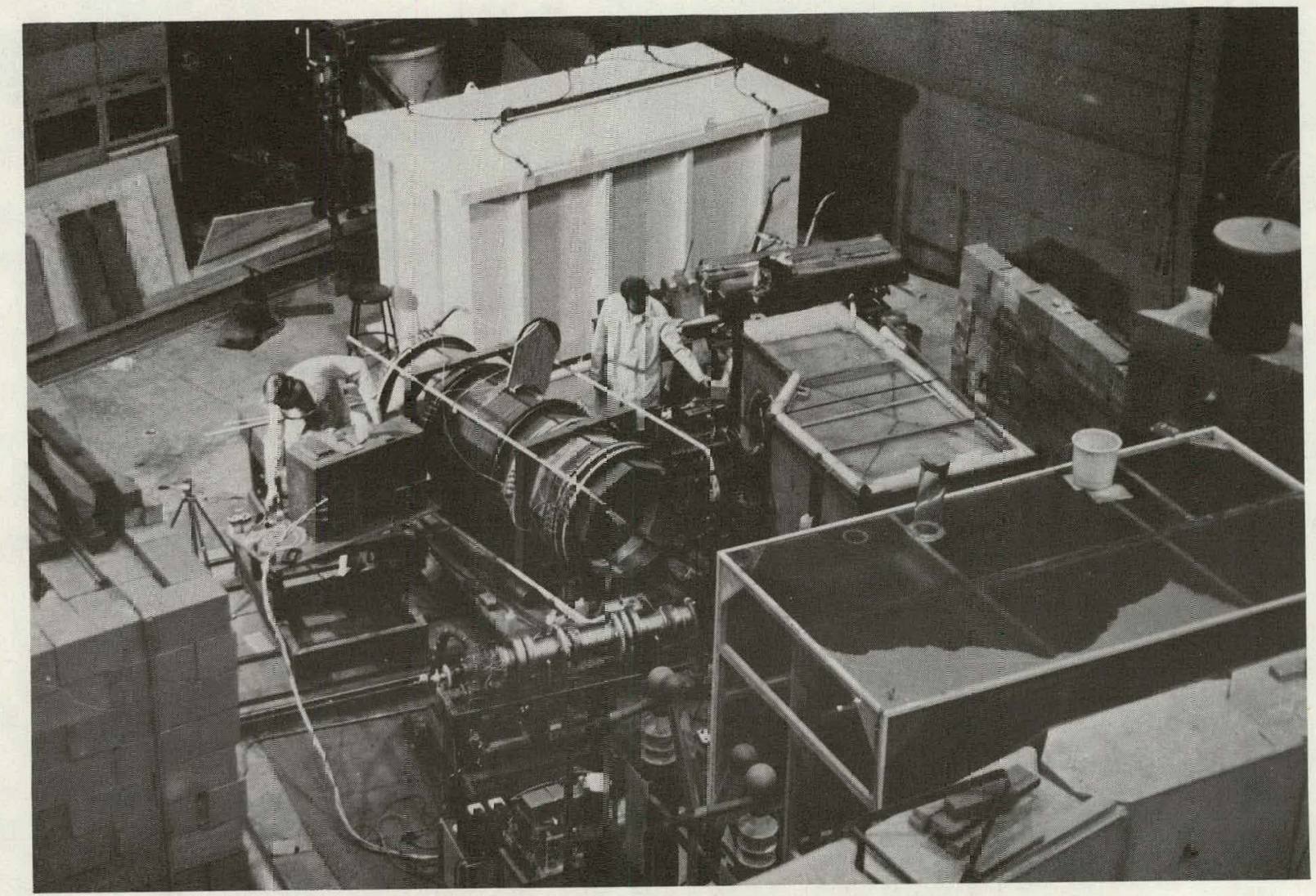

FIGURE 2-4 
CHAPTER 3

SUB-MICROSECOND TRAPPING OF ELECTRON RINGS

IN RECE-Berta IN NEUTRAL GAS 


\section{CHAPTER 3}

\section{SUB-MICROSECOND TRAPPING OF ELECTRON RINGS}

IN RECE-Berta IN NEUTRAL GAS

\subsection{Introduction}

The bulk of the research done on relativistic electron rings has dealt with ring behaviour on time scales of the order of 2-2000 $\mathrm{usec}^{29-31}$ and in some cases (for low- $\zeta$ rings confined in high vacuum), for even longer periods of time. ${ }^{32}$ such time scales are of interest to study the susceptability of the rings to micro- and MHD instabilities, as well as to determine the integrity of the "Absolute Minimum-B" geometry as a plasma containment concept. On the other hand, it is also of physical importance to study the basic mechanism by which the injected beam becomes trapped as an electron ring in the mirror field. That is, what is the dynamic behaviour of the rotating electron beam during the first microsecond, or so, following injection into the confining magnetic well?

As mentioned in Chapter 1, such "fast trapping" studies had been carried out to some extent in the Livermore Astron. 33 However, owing to the limitations on the field-reversal strength of the rings produced in that 
device, the question remained open as to the nature of the trapping process for the strong $(\zeta \sim 50-100 \%)$ electron rings confined in the cornell experiments.

This chapter describes the results of an experimental survey of the trapping phase of the intense electron ring confinement process in RECE-Berta in the presence of a background gas. It was hoped that an understanding of the observed first-order "gas-trapping" phenomenology of strong rings might serve as a basis for understanding electron ring trapping both in a preionized medium (a topic considered in Chapter 5); and at higher injected electron beam energies, as in the RECEChrista experiments. 34

\subsection{Experimental Diagnostics}

Three independent aspects of the beam injection process were studied in this work: (1) the density of the plasma created by beam ionization of the background hydrogen gas, (2) the axial diamagnetic fields created by the circulating electrons, and (3) the axial distribution of the circulating high-energy electron currents. The spatial arrangement of the diagnostics used on RECE-Berta to make these measurements is illustrated in Figure (3-1). The plasma density was obtained using microwave interferometry near the center of the magnetic 
FIGURE 3-1

Diagnostic arrangement used for fast-trapping studies. 


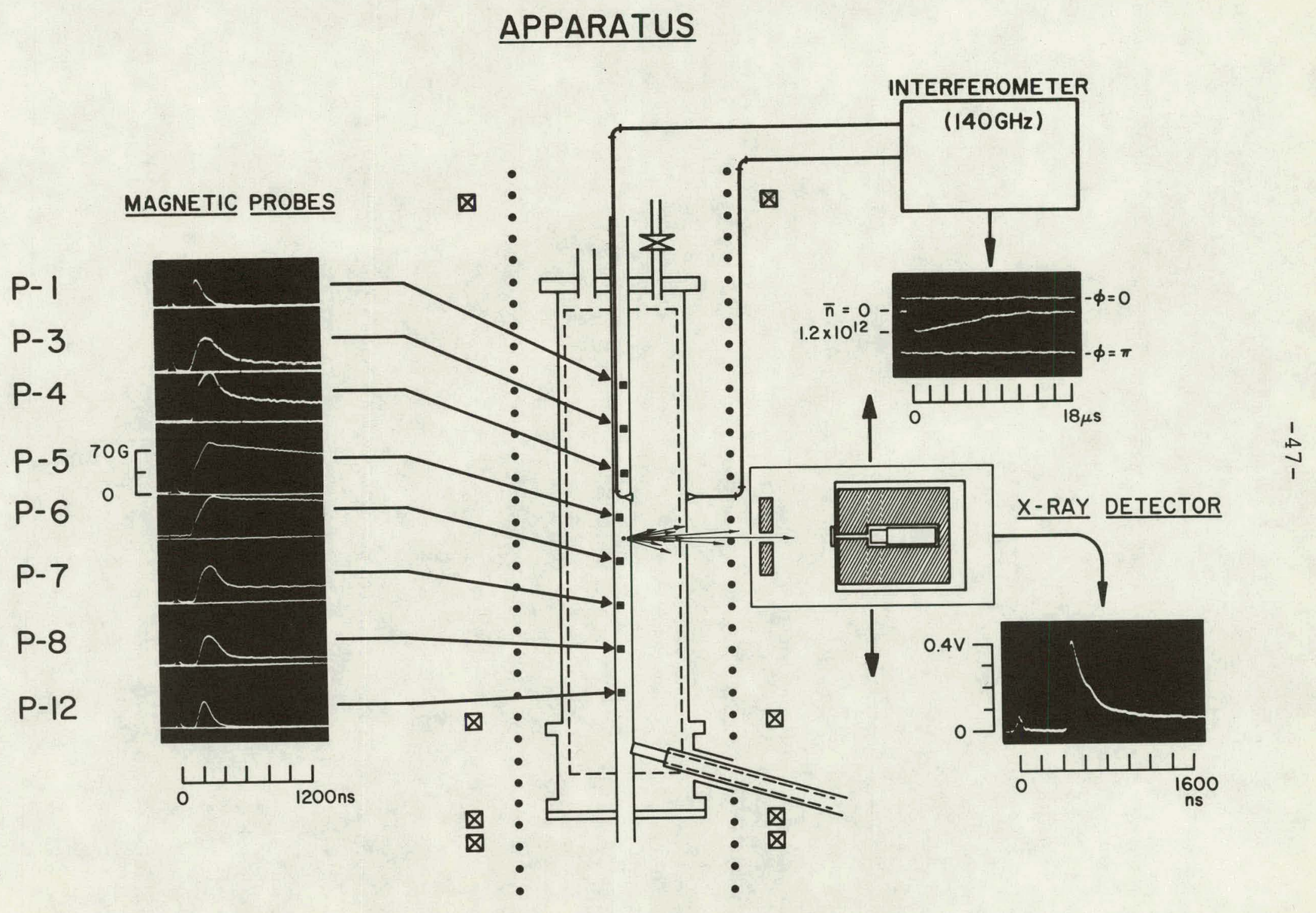

FIGURE 3-1 
well. The diamagnetic fields produced by the electron currents were measured using fast magnetic pickup probes distributed along the machine's axis. The high-energy electron current density monitor was located off to the side of Berta and could be moved parallel to the machine axis to allow for spatially-resolved current measurements.

\subsubsection{Plasma Density Measurements}

Measurements of the beam-created plasma density were carried out using a Mach-zehnder microwave interferometer. The choice of the particularly short wavelength used $(2 \mathrm{~mm})$ was forced by the uncertainty as to the magnitude of the initial plasma density as the electron rings are formed. (For example, the work of McArthur and Poukey ${ }^{35}$ calculated a beam-generated plasma density of the order of $2-4 \times 10^{15} \mathrm{~cm}^{-3}$ using beams of 10-30 kA of electron current injected into 1.5 - 10 Torr of nitrogen.) Thus, it was desirable that the interferometer have the capacity to monitor as high a plasma density as possible which, for $\nu_{0}=140 \mathrm{GHz}(\lambda \simeq 2 \mathrm{~mm})$, corresponds to about $2 \times 10^{14} \mathrm{~cm}^{-3}$.

As discussed in detail by Heald and wharton, 36 the phase of an electromagnetic wave (with no external magnetic fields present) launched through a plasma having a dielectric function 


$$
\varepsilon=c\left\{1-\frac{\omega_{p}^{2}}{\omega_{0}^{2}}\right\}^{1 / 2},
$$

for $v^{2}<<\omega_{p}^{2}$ (where $v$ is the collision frequency and $\omega_{p}$ is the plasma frequency) is changed by an amount

$$
\Delta \phi=\frac{2 \pi}{k_{0}} \int_{0}^{D}\left(1-\left\{1-\frac{n(x)}{n_{c}}\right\}^{1 / 2}\right) d x .
$$

The path of integration is between the transmitting and receiving horns and $n_{c}=\varepsilon_{0} m \omega_{p}^{2} / e^{2}$. If $n(x)<<n_{c}-$ which, as shall be shown, is the case for our experimental conditions--a simple expansion of the integrand in Equation (3.2) yields

$$
\Delta \phi=\frac{e^{2}}{2 \varepsilon_{0}{ }^{m c \omega_{0}}} \int_{0}^{D} n(x) d x .
$$

Putting in numbers, we find that

$$
\int_{0}^{D} n \cdot(x) d x=119 v_{0} \Delta \phi\left(\mathrm{cm}^{-2}\right),
$$

where $\nu_{0}$ is the frequency of the microwave radiation ( $\mathrm{Hz}$ ) and $\Delta \phi$ is the fringe shift (radians).

\subsubsection{Interferometer Configuration}

The position of the interferometer relative to the electron rings trapped in the mirror well is sketched 
in Figure (3-2). As shown, the electronics were shielded from electromagnetic noise with a Faraday cage, the microwave detectors being further screened from burst $\mathrm{x}$-radiation using 2" lead bricks. To minimize electrical interference, the recording oscilloscope was housed inside the copper Faraday cage with the rest of the interferometer electronics and plumbing.

A $25 \mathrm{db}$ receiving horn was mounted on the outside of the vacuum tank and a rectangular hole breached in the copper meshes lining the inside walls of Berta to allow transmission of the microwave signal. A $15 \mathrm{db}$ transmitting horn (chosen for its compact size) was mounted inside a $5.7 \mathrm{~cm}$ diameter Lexan cantilever section. A $3.2 \mathrm{~mm}$ Lucite window in front of this horn prevented electron beam bombardment of the horn and served as a low-loss vacuum interface (the range of $500 \mathrm{keV}$ electrons in Lucite is $1.5 \mathrm{~mm} .^{37}$ ) Both horns were oriented such that the oscillating electric field of the microwave radiation was parallel to the axial magnetic field, thereby exciting only "ordinary" modes in the plasma and justifying the use of Equation (3.1).

Since the physical dimensions of the electron ring (10 cm radius, $20 \mathrm{~cm}$ axial length) are almost two orders of magnitude greater than the $2 \mathrm{~mm}$ wavelength of 


\section{FIGURE 3-2}

Spatial configuration of $2 \mathrm{~mm}$ Mach-Zehnder interferometer with respect to confined electron ring. 


\section{RECE BERTA}

INTERFEROMETER

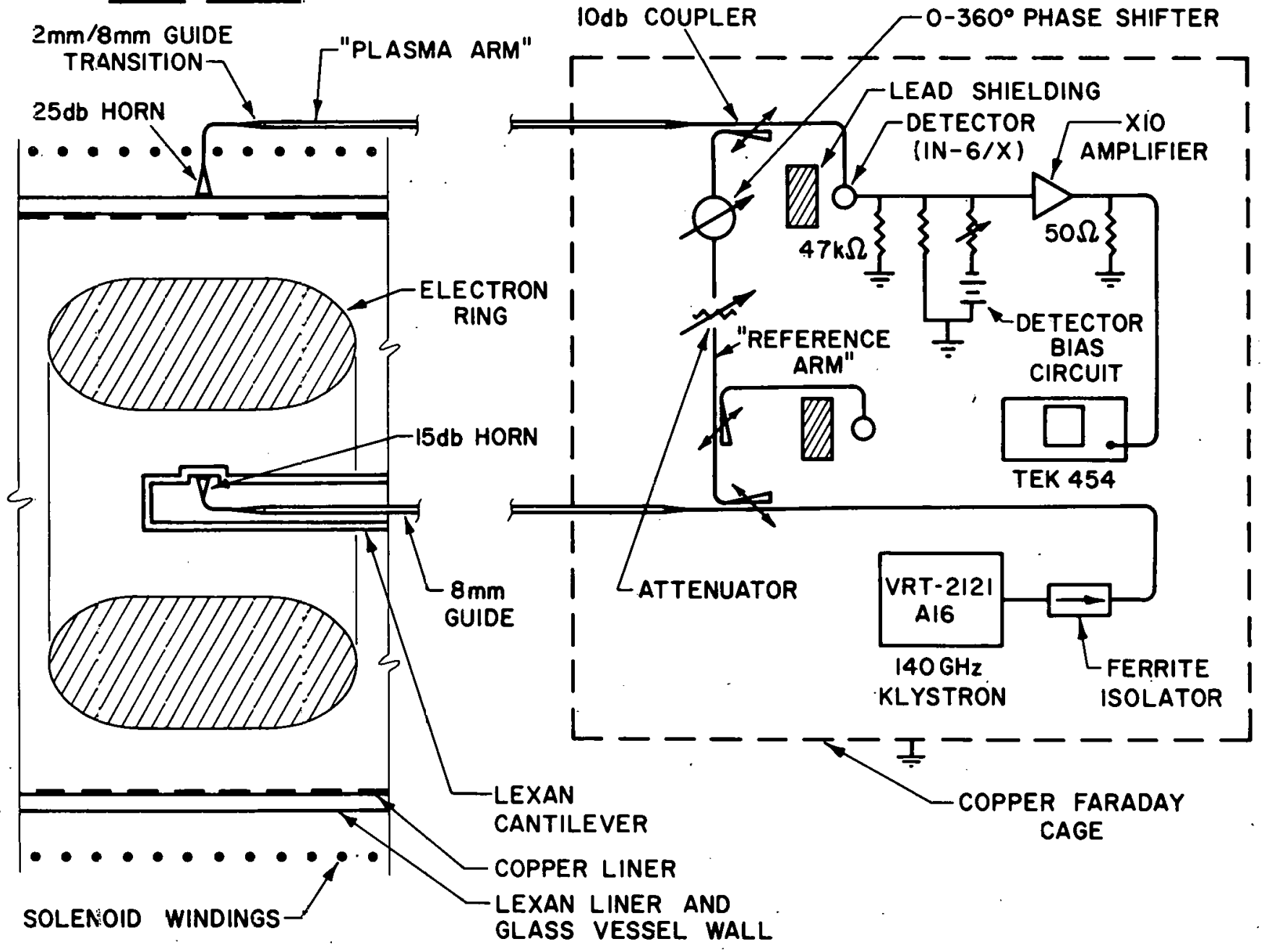


the microwave radiation, for all practical purposes the ring looks like a semi-infinite slab to the radiation, and diffraction effects can be expected to be negligible. ${ }^{38}$ For greater detection sensitivity, and to insure better square-law response of the detector diodes, the detecting elements were biased with a forward current of 3.0. $\mu$ A.

The interferometer response was limited by the high output impedance of the detector diodes (measured to be about $30 \mathrm{k} \Omega$ ) combined with stray capacitance (10-20 pfd) to 300-500 ns. Since the main interest lay in the plasma density as the rings have become established, this response time was found to be adequate for the purposes of this work.

\subsubsection{Experimental System Tests}

Initially, CW-signal tests were conducted which demonstrated that neither mechanical vibrations from the current surges in the solenoid field windings (a definite problem with $2 \mathrm{~mm}$ wavelengths), beam X-radiation, nor electromagnetic noise were contributing factors in the detected signal. Also, transmission tests were conducted to check for signal attenuation in the beam- and.ringcreated plasmas by fully attenuating the reference branch and tuning the reflector voltage for a klystron mode 
maximum. No measurable attenuation was found (with the interferometer purposely detuned to an initial phase shift of $\sim 90^{\circ}$ ), thereby justifying the assumption that $\omega_{p}^{2} \gg v^{2}$, and that dissipative plasma effects were negligible.

For all of the data taken, the densities measured did not exceed $1-2 \times 10^{12} \mathrm{~cm}^{-3}$. Because this represents only a fraction of a fringe-shift at $140 \mathrm{GHz}$, the interferometer was always initially detuned between $45^{\circ}$ and $60^{\circ}$ off-null for greatest sensitivity.

\subsubsection{Magnetic Field Probes}

One of the most useful techniques for observing electron ring behaviour and lifetime is to monitor the diamagnetic fields produced by the rings with magnetic, or "B' probes. 39

\subsubsection{Magnetic Probe Design}

The physical principle underlying the use of these probes is straightforward. As illustrated in Figure (3-3), the probe consists of one or more windings, or loops, of conducting wire placed in a time-varying magnetic field. Using the integral form of Faraday's Law, the total voltage, $v$, which is induced around the windings of the probe is related to the magnetic field, $\vec{B}$, by 
FIGURE $3-3$

(Top) Details of magnetic pickup probe construction; (Bottom) Electrical schematic of probe integrating circuitry. 


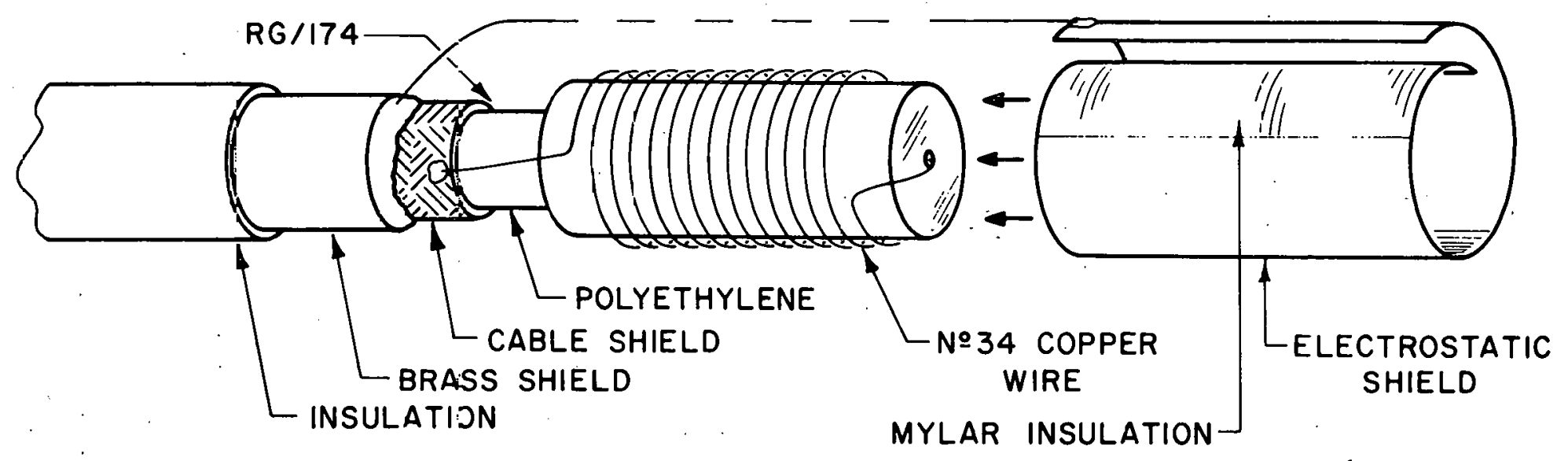




$$
v=-\int_{A} \frac{\partial \vec{B}}{\partial t} d \vec{S}=-n A \frac{\partial B_{n}}{\partial t}
$$

where $A$ is the area enclosed by each winding, $n$ is the number of windings used, and $B_{n}$ is that component of the magnetic field perpendicular to the surface, A. Both $A^{1 / 2}$ and $\ell$, the overall axial length of the probe windings, are assumed to be small compared to dimensions characteristic of spatial changes in the magnetic field. In this work, since only values of $\vec{B}$ were of interest, the signals from these probes were time-integrated to obtain a direct measurement of the magnetic field values. The integration was performed actively using Tektronix "Type o" operational amplifier oscilloscope plug-in units, or (in most cases) passively, using high-frequency resistive/capacitive integration circuits. The passive integrator, illustrated in the lower part of Figure (3-m), produces an output signal, $v_{\text {out }}$ ' related to the time-dependent input signal, $v_{\text {in }}$, as

$$
\begin{gathered}
v_{\text {out }}=\frac{1}{R C} \int_{0}^{t} e^{-\frac{\left(t-t^{\prime}\right)}{R C}} v_{i n}\left(t^{\prime}\right) d t^{\prime} \\
=\frac{1}{R C} \int_{0}^{t} v_{i n}\left(t^{\prime}\right) d t^{\prime}+\frac{1}{(R C)^{2}} \int_{0}^{t} v_{i n}\left(t^{\prime}\right)\left(t-t^{\prime}\right) d t^{\prime}+\ldots
\end{gathered}
$$


Typically, the RC-time constants of the integrators designed for this work were between 30 and 100 times longer than the observed signal pulsewidths, leading to corrections to the first term in the expansion of Equation (3.6) of the order of $1-28$.

The magnetic response time (or "penetration time") of the probes (i..e.., the time required for the magnetic field in the volume circumscribed by the probe windings to reach a value of $1-(1 / e)$, or $\sim 63 \%$, of its final value) is given by their " $L / R$ " time, where $L$ is the probe inductance and $R$ is the total circuit resistance across the windings. For this work, the minimization of the L/R time implies either few windings (producing both lower inductance and lower signal levels) or a large parallel resistance (which may not be compatible with the limitations of the $50 \Omega-100 \Omega$ characteristic impedance of standard transmission cable and might therefore result in "loading" of the probe by the [terminated] cable anyway).

\subsubsection{Physical Construction}

The actual probes used, shown in Figure (3-3), consisted of 40 and 80 turns (for the "fast" and "slow" probes, respectively) of AWG \#34 (0.14 mm diameter) formvar copper wire wound on polyethylene spindles $2.95 \mathrm{~mm}$ 
in diameter. To avoid picking up stray fields perpendicular to the probe axis, the center wire returns through the center of the spindle and connects to the coaxial cable center conductor (located several probe diameters away from the rear end of the probe winding because of limiting field penetration effects in the solid copper jacket of the coaxial transmission cable used). The probe cross-sectional area, A, is small, and, should the center return wire have a cross-sectional area which is an appreciable fraction (108) of A, the measured field will be seen to rise slowly (and erroneously) as an otherwise constant magnetic field penetrates the wire. (For example, \#22 wire; the size of the center conductor of RG/174 coaxial cable, has a diameter of $0.635 \mathrm{~mm}$. The magnetic field penetration time for this size of wire [roughly given by $r^{2} \mu_{0} \sigma$ ] is about 8 usec.) The \#34 wire used for the probe windings produced a negligible error of this kind. The inductances of the probes were 1.7 $\mu \mathrm{H}$ and $6.5 \mu \mathrm{H}$ for the fast and slow probes, respectively. The transmission cables were matched at the integrators with $50 \Omega$, giving $L / R$ times of 35 (and 130) ns for the fast (and slow) probes. Integrators with RC constants of 100,300 , and $600 \mu$ sec were used in the experiments. 
The probes were calibrated in a pulsed, uniform solenoidal field of 150 Gauss driven by a variable-width rectangular current pulse with 5 usec $<\tau<150$ usec. For reference, the overall probe calibrations were $3.4 \mathrm{G} / \mathrm{mV}$ for the fast probes (into 100.0 usec integrators) and $5.1 \mathrm{G} / \mathrm{mV}$ for the slow probes (into $300.0 \mu \mathrm{sec}$ integrators). Electrostatic shielding against the extreme electrical noise environment was accomplished, as shown in Figure $(3-3)$, by placing a turn of $0.076 \mathrm{~mm}$ brass shimstock around the probe windings, with the ends of the shield electrically insulated from each other to allow for magnetic field penetration. The shield was then grounded at one point to the coaxial cable shielding. For most of the work, the magnetic field probes were arrayed axially along the center of Berta, held inside a $5.72 \mathrm{~cm}$ diameter Lexan tube which ran the full length of the machine. The probes were separated from one another by plastic spacers to allow full field penetration and to minimize cross-interference between probes.

\subsubsection{High-Energy Electron Current Monitor}

One of the most direct methods by which high energy electrons may be detected is through the Bremsstrahlung they produce upon striking a high-z target. In a manner 
similar to that employed to make ring electron energy measurements (to be discussed in Chapter 4), this idea was put to use in this work by suspending a wire target vertically from the top of the vacuum vessel to the machine axis. The circulating high-energy electrons would then strike the wire, generating $\mathrm{x}$-rays which could be monitored by a remote detector.

\subsubsection{Target Geometry and Configuration}

The choice of a target and detector combination to use for this type of measurement is governed by several requirements. First, the shortest characteristic time scale in the events to be measured is that of the beam current risetime, about 60 ns. Since this measurement is not designed to measure the details of the trajectory of the helical beam on injection, we may safely take a response time of the order of $50 \mathrm{~ns}$ as a minimum requirement for the monitor. However, this response time rules out the use of sodium iodide ( $250 \mathrm{~ns}$ decay time $\left.{ }^{40}\right)$, and we are limited to plastic scintillators (response time of 1-2 $\mathrm{ns}^{41}$, and their inherently low detection efficiency. Thus, one must monitor a copious flux of $x$-rays (í.e... use a larger target or a larger detector) to obtain reasonable counting statistics. This necessitates a 
trade-off between the desired high $\mathrm{x}$-ray fluxes and the need not to perturb the injected beam physically.

Experimental X-ray measurements with tungsten wires of various sizes ultimately led to the use of a 0.02 cm diameter filament as a target. For null-test purposes, it was to be necessary to remove the target often from the interior of the vacuum vessel. To this end, a simple sliding carriage mounted inside a $1.6 \mathrm{~cm}$ diameter pyrex glass rod was used to "remove" the target from the machine. The wire hung from a loop at the end of a long $3.2 \mathrm{~mm}$ diameter plastic rod placed inside the glass rod, with a slug of iron attached to the other end of the plastic rod. One could engage the iron slug mechanically with a small magnet held near the outside wall of the glass tube and, by moving the magnet (and slug) towards the closed end of the glass rod, draw the tungsten filament up into the rod, out of the way of the beam. (The small iron slug was not affected by the pulsed fringing mirror fields.) When in the "down" position, the tungsten wire hung from the top of the vessel to the top of the Lexan tube, and could be located at various axial positions by sliding the glass support rod through its vacuum fitting in the endplate of the vessel. 


\subsubsection{Detector Configuration}

The detector consisted of a $5.08 \mathrm{~cm} \times 5.08 \mathrm{~cm}$ right cylindrical plastic scintillator composed of $10 \%$ lead-doped (by weight) Pilot B (Nuclear Enterprises, Inc. scintillator compound "PS/ $\mathrm{Pb}$ ") mounted to the face of a ten-stage EMI-9856 venetian-blind photomultiplier tube. The detector was wrapped in magnetic shielding and housed in a light-tight case made of $3.2 \mathrm{~mm}$ thick alumina. The $10 \%$ lead doping enhances the absorption of X-rays in a detector otherwise severely limited in detection efficiency owing to the small value of the compton cross-sections for carbon and hydrogen at energies in the range of $100-500$ kev. Overall, the addition of the lead increased the absorption efficiency of $300 \mathrm{keV} X$-rays by 418 , and of $500 \mathrm{keV} x$-rays by 228.42

The detector was housed in a lead chamber which provided at least $15 \mathrm{~cm}$ of lead shielding in all directions except to the front face of the detector. This facet of the scintillator was collimated to view the Bremsstrahlung from the wire target as shown in Figure (3-4). The collimation geometry was designed to optimize $x$-ray "signal-to-noise". Care had to be taken that the detector was collimated to view the target exclusively in a way which subtended as large a solid angle as possible 
$-64-$

FIGURE 3-4

Collimation geometry for high-energy electron current monitor. 


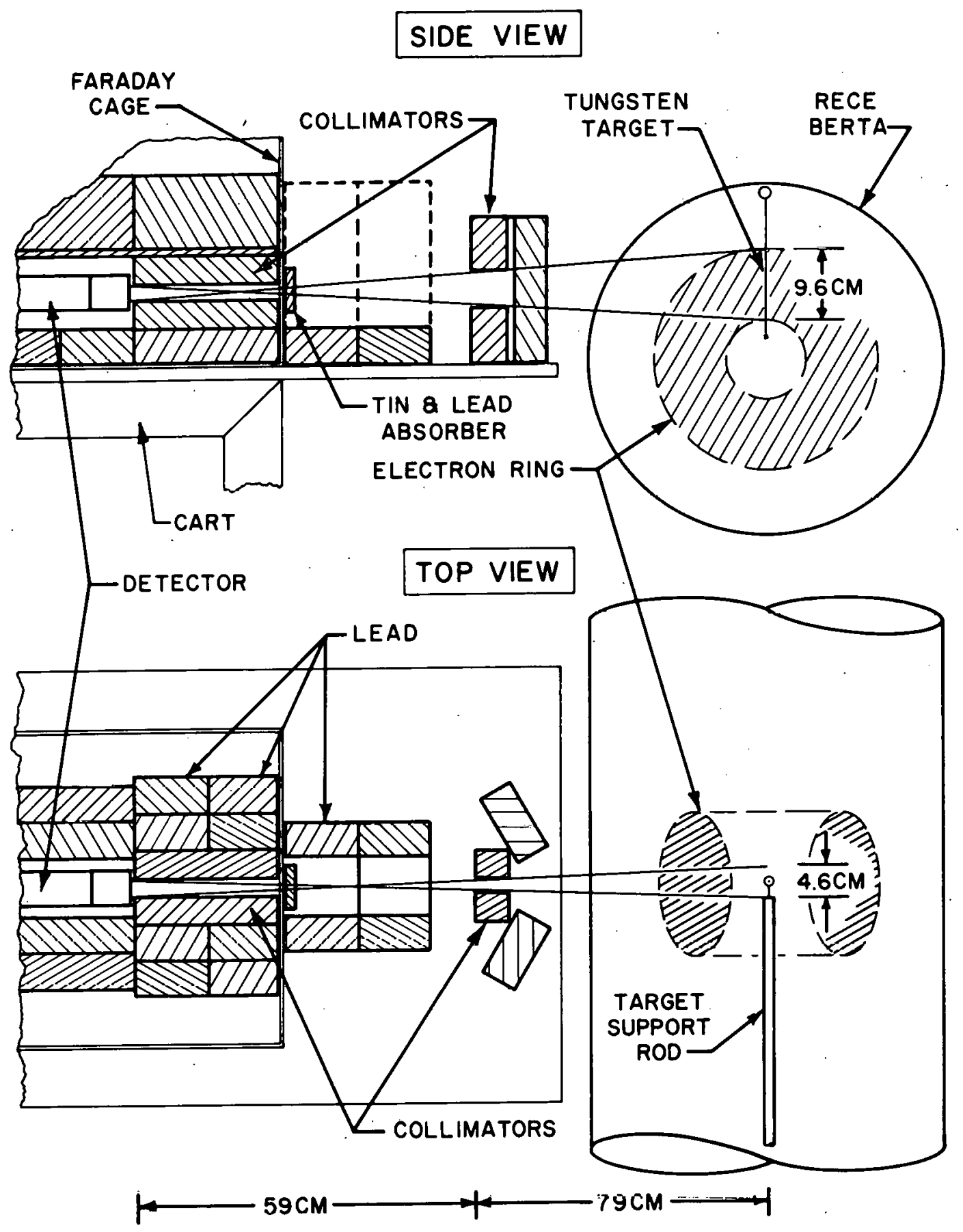

FIGURE 3-4 
from the wire. As illustrated in Figure $(3-4)$, the lead collimator limited the acceptance area in the plane of the target to a rectangular field, $4.6 \mathrm{~cm}$ wide by $9.6 \mathrm{~cm}$ high. The detector subtended a solid angle of $3 \times 10^{-4} \mathrm{Sr}$ from the center of the wire. For reasons discussed in greater detail in Chapter $4,1.9 \mathrm{~g} / \mathrm{cm}^{2}$ of tin and $2.52 \mathrm{~g} / \mathrm{cm}^{2}$ of lead were placed in front of the collimator to suppress radiation below $150 \mathrm{keV}$ (cf. Figure (4-15)). The entire detector/collimator assembly was mounted on a cart which travelled on a pair of $10 \mathrm{~cm}$ wide rails parallel to the axis of Berta. This allowed (fairly) easy relocation of the detector to view the target at various axial positions in the machine. (The detector and collimator assembly weighed about 700 pounds.)

To assure both linear operation and optimal risetime characteristics of the photomultiplier tube, a nonlinear dynode chain voltage division ratio of $1: 2: 4: 6: 3$ was used on the last four dynode stages of the photomultiplier, with $R_{0}=240 \mathrm{k} \Omega$ used in the linear section of the divider. ${ }^{43}$ Effective photocathode-to-first dynode acceleration was provided by three $150 \mathrm{~V}$ zener diodes. To insure constant final-stage accelerating potentials, the last four dynode stages were provided with substantial capacitive loading $\left(c_{7,8}=0.01 \mu \mathrm{fd}, c_{9,10}=0.02 \mu \mathrm{fd}\right)$. 
(Thus, a sustained output current of $10 \mathrm{~mA}$ for $1.5 \mu \mathrm{s}$ would only decrease the voltage on the last two stages by less than 1\%.)

The detector output was direct-coupled into a matched cable load of $50 \Omega$, which necessitated holding the photocathode at $-\mathrm{HV}$ and wrapping the detector in Mylar to prevent leakage currents through the glass envelope of the photomultiplier (the interior magnetic shield floated with the tube potential at the photocathode).

Using a $\mathrm{Co}^{60}$ source, the risetime of the detector was measured to be $\sim 13 \mathrm{~ns}$, with a pulse FWHM $\sim 30 \mathrm{~ns}$, in the anode-cathode voltage range investigated $(-1050$ -1600 VDC).

The overall operating characteristics of the detector were tested for possible space-charge limiting effects using a $7 \mu \mathrm{Ci} \mathrm{Cs}^{137}$ source (solitary gamma emitter, 0.661 $\mathrm{MeV})$ located $15 \mathrm{~cm}$ from the front face of the detector to avoid pulse pile-up. As mentioned above, almost all photon interactions with the plastic scintillator are Compton scatterings. Hence, the output from such a detector irradiated by a monoenergetic $\mathrm{X}$-ray source consists of the compton spectrum up to the compton "edge" energy (cf. Appendix.III-6). The technique in making 
these test measurements hinged on measuring the "edge" energy by taking time-integrated photographs of the output pulses from the photomultiplier tube: a well-defined envelope to the pulses could be easily discerned, with the maximum of the envelope corresponding to the maximum photon energy transfer to the scintillator. The time of exposure was the same for all oscillographs.

An example of the detector output signal illustrating the spectrum of pulse-heights, as well as the detector risetime and pulse FWHM, using $\mathrm{Co}^{60}$ as a radiation source, is shown in Figure (3-5).

The result of these saturation tests is shown in Figure (3-6). As evident in the figure, no saturation effects could be found for pulsed currents up to $12 \mathrm{~mA}$. The manufacturer's specifications on this particular tube for single-pulse operation indicate that spacecharge saturation effects begin to take place for current levels in excess of $20 \mathrm{~mA}{ }^{44}$ Typically, the signal levels obtained during the experimental work were in the range of 10-15 mA at a tube bias voltage of -1000 VDC.

\subsubsection{Detector Calibration}

It was possible to make a rough calibration of the measured $X$-ray signals directly in terms of the densities of fast electrons. This was done by using the electron rings themselves as a "calibration source", assuming that 


\section{FIGURE $3-5$}

Detector current waveforms resulting from full-body irradiation of $\mathrm{Pilot} B$ detector by $\mathrm{CO}^{60}$ source; time of photograph exposure $=12 \mathrm{sec}$. 


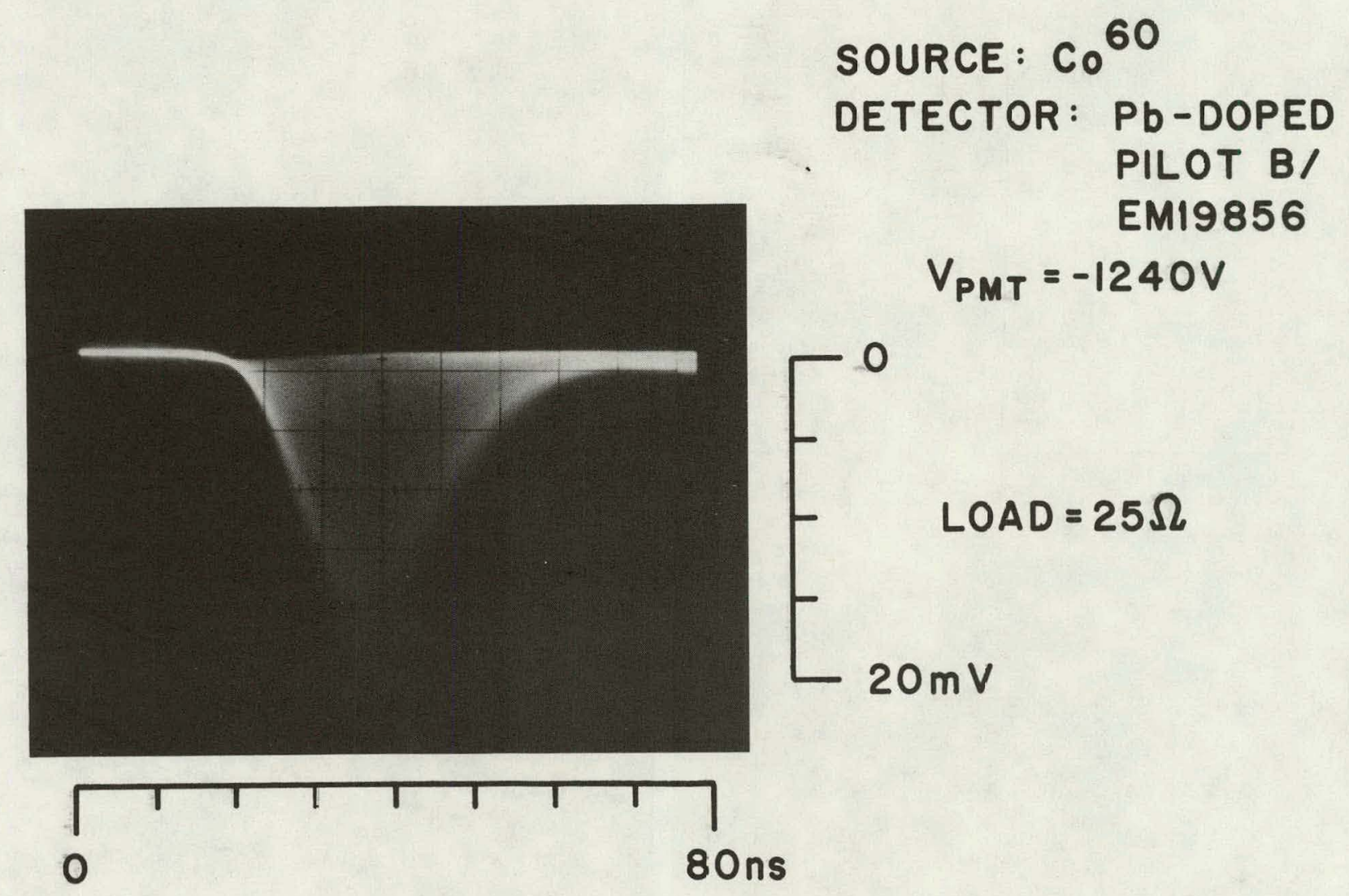


FIGURE $3-6$

Gain-saturation tests on fast X-ray detector. 


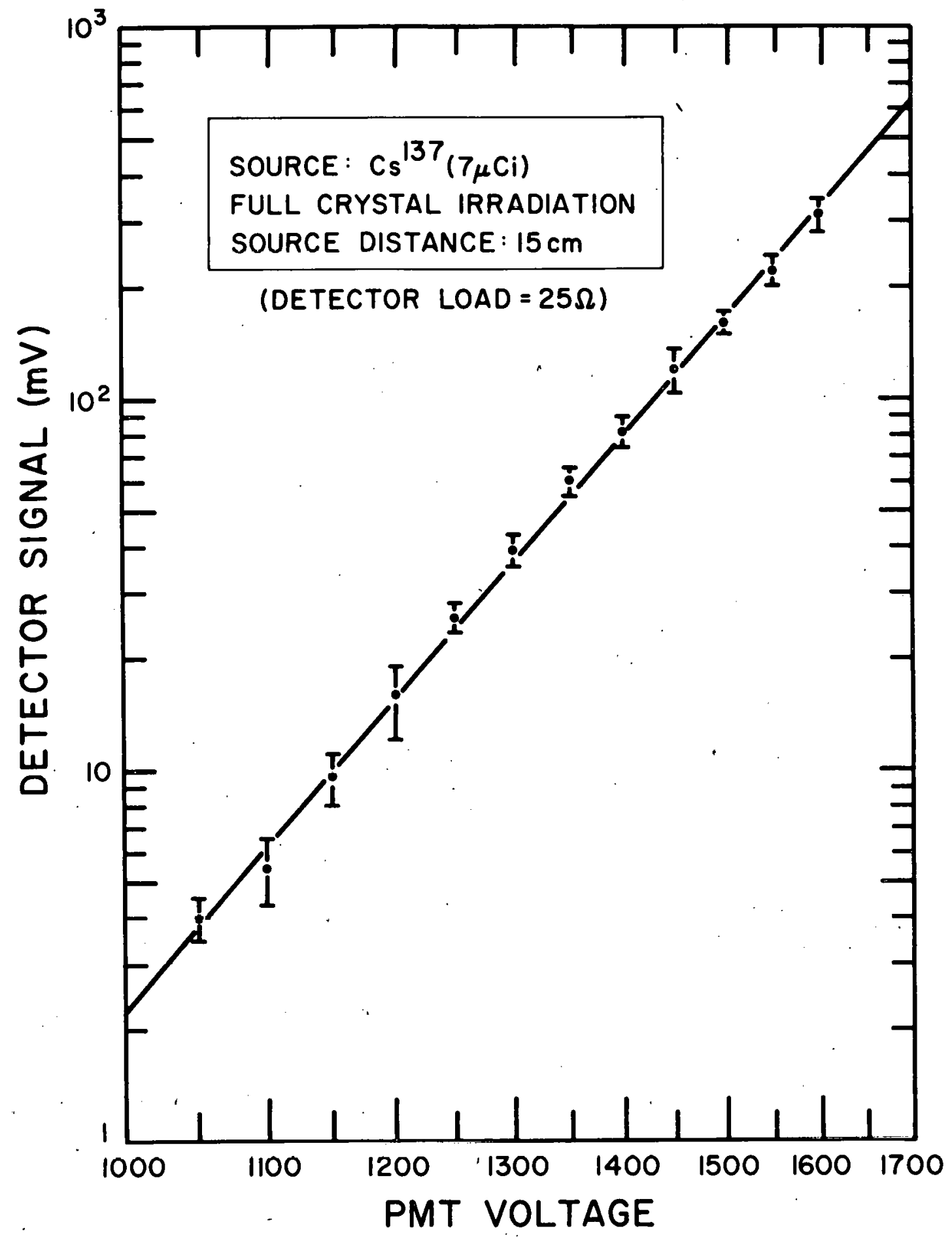

FIGURE $3-6$ 
the detected $\mathrm{X}$-ray signal was due entirely to ring electrons striking the wire target (․․…, neglecting any low-level background signal, etc., which, as will be discussed in Section 3.3.3, is a good assumption). The experimental procedure was to locate the tungsten target directly in the center of the electron rings (that location having been determined by the magnetic field profiles). Direct comparisons were then made between the $X$-ray signals and the output of the magnetic field probe located at the same axial position as the target at times greater than 500-700 ns after beam injection, when all ring motion and plasma currents had subsided. A plot comparing the $x$-ray signals directly against the probe signals is shown in Figure (3-7) for several shots at a number of different time intervals after beam injection. From the plot, we obtain the relationship that $\mathrm{B}_{z} \sim$ 2.8I PMT (Gauss), where $B_{z}$ is the ring axial diamagnetic field at the axial center of the ring, and $I_{P M T}$ is the $\mathrm{X}$-ray detector current (mA). Presuming that the ring current channel is roughly rectangular in cross-section, with an axial length of $\sim 20 \mathrm{~cm}$, a radial thickness of - $10 \mathrm{~cm}$ (this has not been measured experimentally, but is based on end-on photographs of the electron rings) 45 and a mean radius of $\sim 10 \mathrm{~cm}$, we find the fast electron 


\section{FIGURE $3-7$}

$\mathrm{x}$-ray detector current versus axial diamagnetic field signals for confined rings on-center with the tungsten target as a function of time. - Individual shot numbers are indicated. 


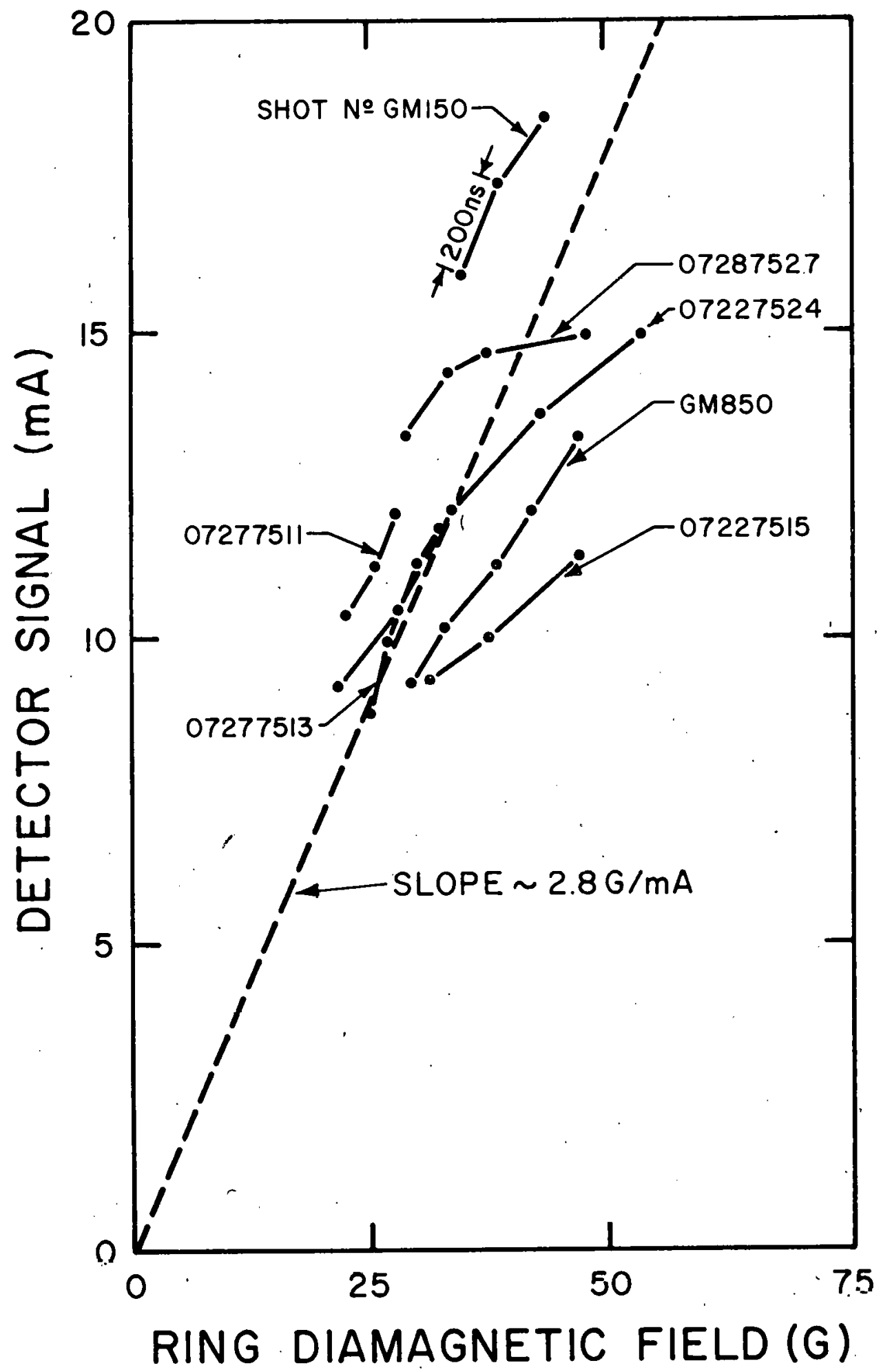


density circulating in the ring to be $\mathrm{n}_{\mathrm{e}}-1.1 \times 10^{6} \mathrm{I}$ $\left(\mathrm{cm}^{-3}\right)$, where $I$ is the circulating ring current in amperes. After correcting for induced liner currents, I may be expressed in terms of $B_{z}$ (cf. Section 4.8.2) and combined with the above expression for $B_{z}$ in terms of $I_{P M P}$ to give the fast electron current density in the rings as a function of $x$-ray detector current, namely,

$$
\mathrm{n}_{e} \sim 8.5 \times 10^{7} \mathrm{~cm}^{-3} \mathrm{~mA}^{-1}
$$

with an overall accuracy of a factor of 2-3.

To check on the magnitude of the proportionality in Equation (3.7), a first-order calculation was performed to estimate the size of the detector output current signal for a given electron ring circulating current density. The details of the computation may be found in Appendix IV, with the result being that $\mathrm{n}_{\mathrm{e}} \sim 3 \times 10^{7} \mathrm{~cm}^{-3} \mathrm{~mA}^{-1}$; that is, the first-order estimate of the constant of proportionality is about a factor of two lower than the experimentally-measured value, which is certainly within the margin of error in the calculation.

\subsubsection{Detection Statistics}

An estimate of the expected signal fluctuations arising from counting statistics in the measurement may be made from data such as that shown in Figure (3-5). 
The 0.511 MeV X-radiation "line" from a $\mathrm{Na}^{22}$ source was monitored with the detector biased at the same highvoltage photomultiplier setting as used in the experimental work $(-1000 \mathrm{VDC})$. The height of the "edge" pulse described above was found to be about $1.6 \mathrm{mv}$. We assume that the average pulse height is about half the maximum "edge" pulse height for the incident $\mathrm{x}$-ray energy concerned, $\underline{i}$.e... amounting to an assumption that the compton spectrum is essentially rectangular in shape which, as discussed in Appendix III-7, is a pretty good approximation in the energy range of interest. Since the maximum $\mathrm{X}$-ray energy is $\mathrm{E}_{\mathrm{O}} \sim 500 \mathrm{keV}$, and the actual signals measured during the experimental runs were of the order of $300 \mathrm{mV}$, we find a (minimum) counting rate of approximately 300-400 gammas per 30 ns pulsewidth. This gives an anticipated statistical fluctuation of no more than 68 in the signal levels, which is acceptable for the purposes of this work.

\subsection{Experimental Trapping Results}

\subsubsection{Plasma Density Measurements}

Measurements of the plasma density were taken at the trough of the magnetic mirror well, where the electron rings are formed. In order to measure plasma 
densities near the axial edges of the electron rings, the strength of the mirror fields upstream and downstream were varied to force the rings to set up at different axial locations, while the interferometer remained stationary. Measurements were made at two background gas pressures, 410 and 140 mTorr hydrogen. In addition, data was collected for single electron beam transits of the tank (by disconnecting the downstream mirror).

Data representative of the plasma densities observed with a single transit of the electron beam (obtained by removing the downstream mirror field), as well as the case of a trapped ring, are shown in Figure (3-8). The background gas pressure for the data shown is 410 mTorr $\mathrm{H}_{2}$. With the mirrors disconnected, the average plasma density (assuming a $10 \mathrm{~cm}$ wide plasma channel) is coon to rise to $1.5 \times 10^{12} \mathrm{~cm}^{-3}$ in aboút one microsecond, followed by a decay to zero in about four microseconds. The magnetic probes indicate a circulating electron current that persists for no longer than a few hundred nanoseconds. When the mirror field is connected, on the other hand, a 708 field-reversing electron ring is confined in the mirror well for about 9 usec. The plasma density once again rises to about $1.5 \times 10^{12} \mathrm{~cm}^{-3}$ and 


\section{FIGURE $3-8$}

Characteristic interferometer responses:

(Top) without a downstream mirror, $\underline{i}$.e.., no

confined ring, single pass of spiral

electron beam;

(Center) with a strong $(\zeta \sim 75 \%)$ confined electron ring;

(Bottom) with a confined ring and initial phase of. interferometer advanced by about $270^{\circ}$. 

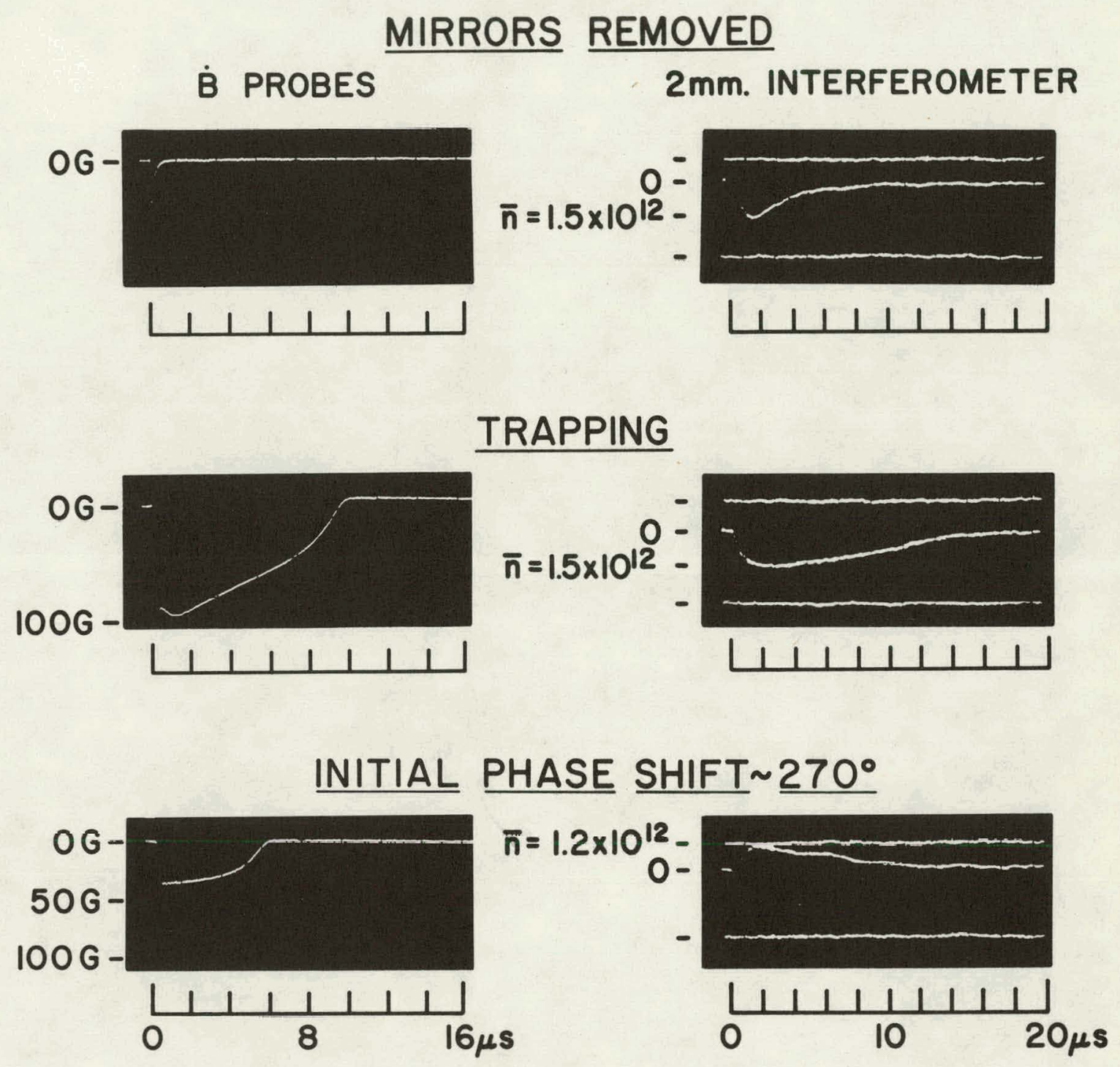
decays smoothly to zero in about 13-15 $\mu$ sec.

In light of the fact that only fractional fringes were being measured, one further check was made to insure the integrity of the observation. The interferometer was detuned to an initial phase shift of about $270^{\circ}$ and measurements made on the plasma. Given this initial baseline phase shift, the signal should appear almost identical to the results obtained with an initial phase shift of about $90^{\circ}$, but inverted--which was indeed the case, as shown in the lower part of Figure $(3-8)$.

A note on the manner in which the data for these fractional fringe shifts was reduced mathematically is presented in Appendix $I=1$.

The plasma density as a function of time for a number of trapped electron rings is shown in Figure (3-9). The shot-to-shot variation in these data is indicated by the error bars on the plot. Although some dependence of density on initial $\zeta$ may be found, by and large, for layers trapped directly between the horns, the densities invariably rose to $1.4-1.6 \times 10^{12} \mathrm{~cm}^{-3}$ in about a microsecond and then exhibited, universally, a fairly linear decay (which generally followed the decay of ring diamagnetism in profile) at the rate of about $1.3 \times 10^{11}$ $\mathrm{cm}^{-3} \mu \mathrm{sec}^{-1}$. At $7-10 \mathrm{~cm}$ from the axial center of the 
FIGURE $\quad 3-9$

Integrated plasma line density as a function of time for various confined electron rings as inferred from interferometer data. 


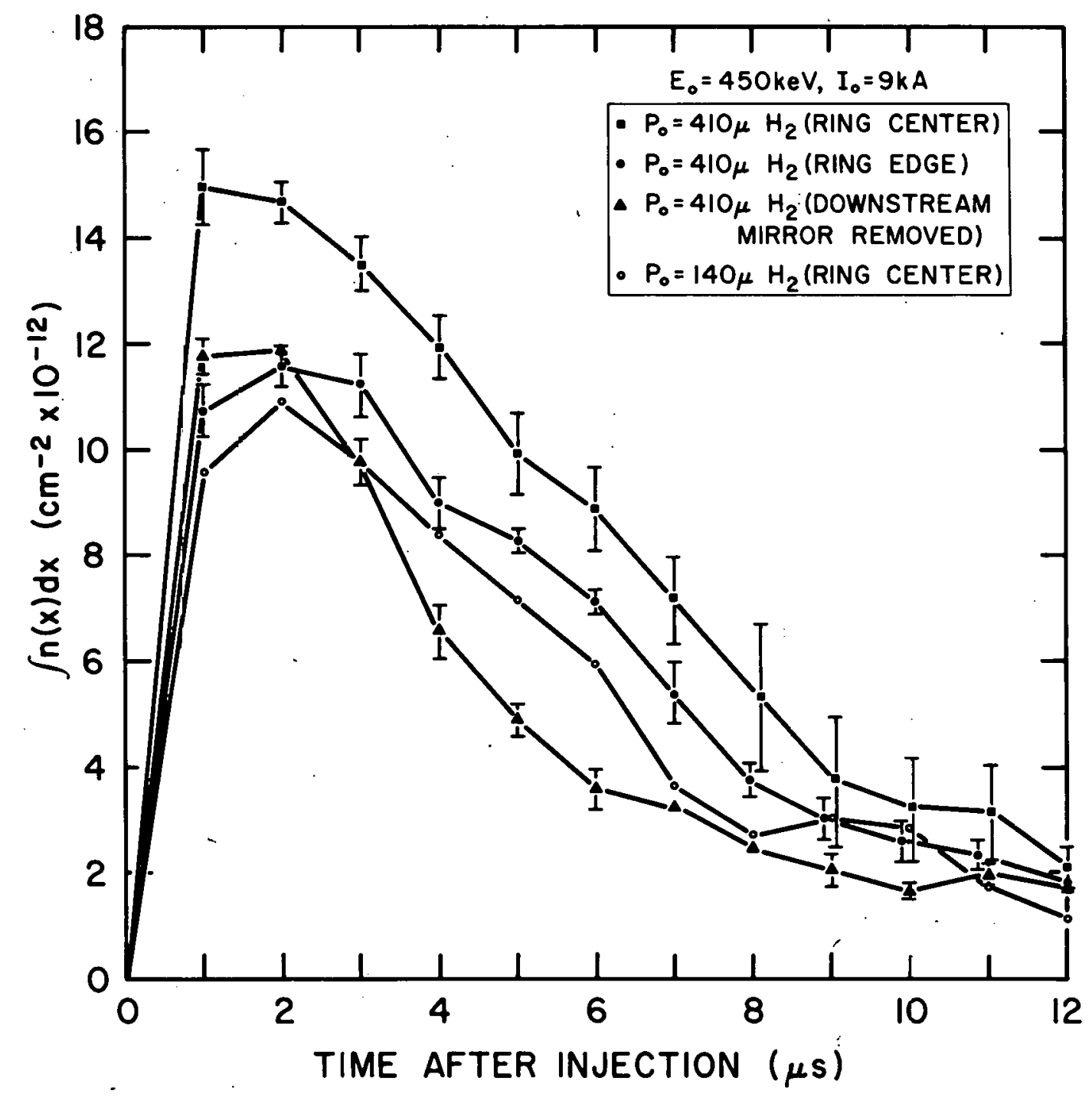


ring, the maximum plasma density was found to be slightly lower $\left(1.0-1.2 \times 10^{12} \mathrm{~cm}^{-3}\right)$, but decayed at about the same rate as the plasma densities near the ring center. It is also interesting to note that, as shown in Figure (3-9), the plasma density observed with the rings trapped in 140 mTorr hydrogen was only about 108 less than that found in rings of comparable circulating current trapped at $410 \mathrm{mTorr}$ hydrogen.

As mentioned earlier, part of the microsecond risetime of the plasma density_can be attributed to the interferometer electronics. However, in this experiment there is no guarantee that the injected electron beam will initially pass directly between the horns of the interferometer. Hence, it is not clear that one should necessarily see a plasma density risetime commensurate with the beam ionization rate (which, at $140 \mathrm{mTorr}_{2}$, is about $35 \mathrm{~ns}$ and, at $410 \mathrm{mTorr} \mathrm{H}_{2}$, is about $10-15 \mathrm{~ns}^{46}$ ). some efforts were made to see if the beam could be made to pass between the horns of the interferometer and a rapid buildup observed. By shifting the mirror fields, for example, the data of Figure (3-10) was obtained, where a plasma buildup in an interval of the order of a couple of hundreds of nanoseconds could be seen. This gives confidence that the microsecond buildup times are 
$-85-$

FIGURE 3-10

Fast-rising interferometer response, indicating probable passage of injected beam between interferometer horns. 
SHOT N2 09267520

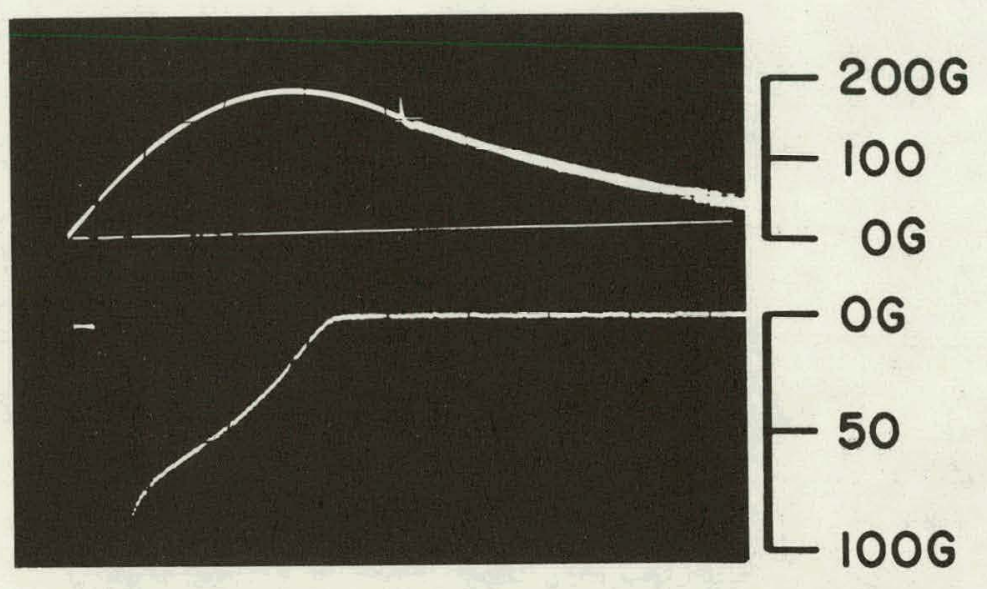

\author{
SOLENOID \\ FIELD
}

(5ms/div)

PROBE NNI

$(100 \mathrm{~cm})$

$(2 \mu s / d i v$.
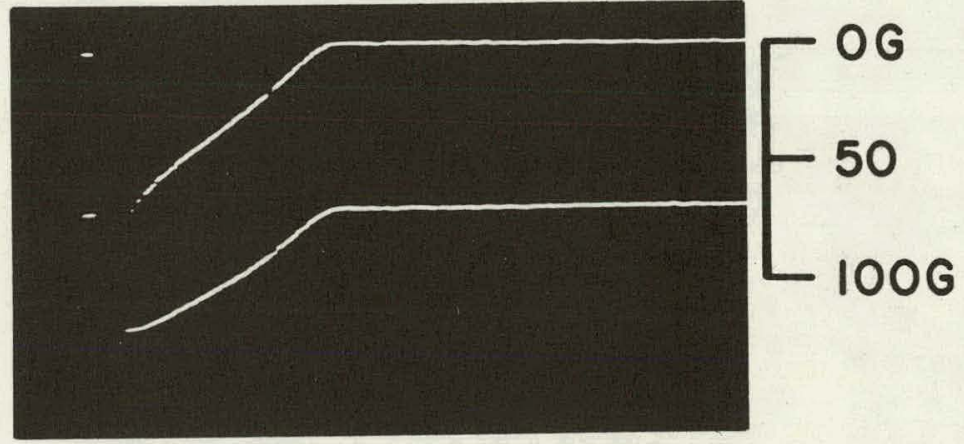
PROBE N 2 $(109 \mathrm{~cm})$

PROBE N23

$(117 \mathrm{~cm})$

$(2 \mu s / d i v$.

\title{
PLASMA DENSITY
}

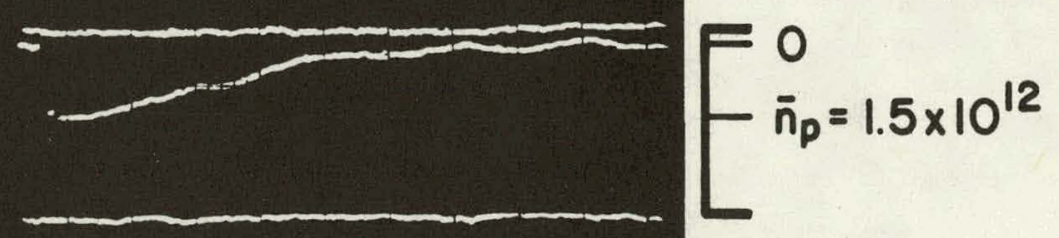

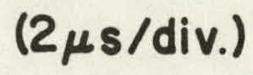


due in part to plasma transport effects, either as the ring sets up, or as plasma diffuses away from the local regions where it was produced by the beam.

Finally, note should be taken that, in all of the density measurements, the measured plasma density (1-2 $\times 10^{12} \mathrm{~cm}^{-3}$ ) was always much less than the cutoff density $\left(\sim 10^{14} \mathrm{~cm}^{-3}\right)$. Hence, the expansion used in the integrand of Equation (3.2) to obtain Equation (3.3) was justified. Also, for $n_{p} \sim 10^{12} \mathrm{~cm}^{-3}, \omega_{p} \sim 5.7 \times 10^{10}$ $\mathrm{rad} / \mathrm{sec}$. As shall be shown in section 3.4, the collision frequency in the plasma is about $v_{c}-10^{9} \mathrm{sec}^{-1}$. Thus, $\nu_{c} \ll \omega_{p}$, and the simplified formulation used for the plasma dielectric function in Equation (3.1) is found quantitatively to be a good approximation, in corroboration with the signal attenuation tests described in Section 3.2.1.2.

\subsubsection{Diamagnetic Field Measurements}

Data was taken on the axial diamagnetic field transients during the first microsecond after injection of the electron beam at backfill pressures of 150, 210, $340,420,530,600,800$, and $910 \mathrm{~m}$ Torr hydrogen. Data from thc array of fuurteen probes distributed along the axial length of the mirror well was reduced in the form of plots displaying the diamagnetic field as a function 
of axial position and parameterized to time intervals following the onset of diode current. A typical plot of this kind is shown in Figure (3-11) for the case of a ring trapped in 910 mTorr of hydrogen.

On the left-hand side of Figure $(3-11)$ are displayed the oscilloscope traces from the probe array. The sweep is 200 ns per division, with all traces initialized by a timing fiducial mark. The axial positions of the probes are indicated to the left of the oscillographs, in centimeters from the downstream end of the solenoid magnetic field windings of Berta.

Characteristically, the signals from probes located toward the ends of the mirror trap show a single, rapidly-rising spike (risetime comparable to beam current risetime) which promptly falls to zero in a few hundreds of nanoseconds. However, as one moves towards the center of the mirror well, one finds those same spikes followed by a persisting, slowly-decaying signal which indicates the presence of the electron ring. The significance of these features is illustrated in the plot on the righthand side of Figure (3-11).

At times shortly after commencement of the electron beam current (75 - 125 ns in Figure (3-11)), inspection of the plot reveals a fairly uniform diamagnetic profile over the length of the mirror trap. As time 
FIGURE $3-11$

Sample oscilloscope traces from magnetic probe array and reduced data parameterized to time after beam injection, for electron ring confined in 910 mTorr hydrogen. 


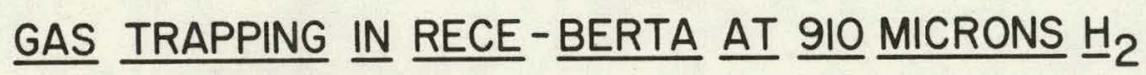

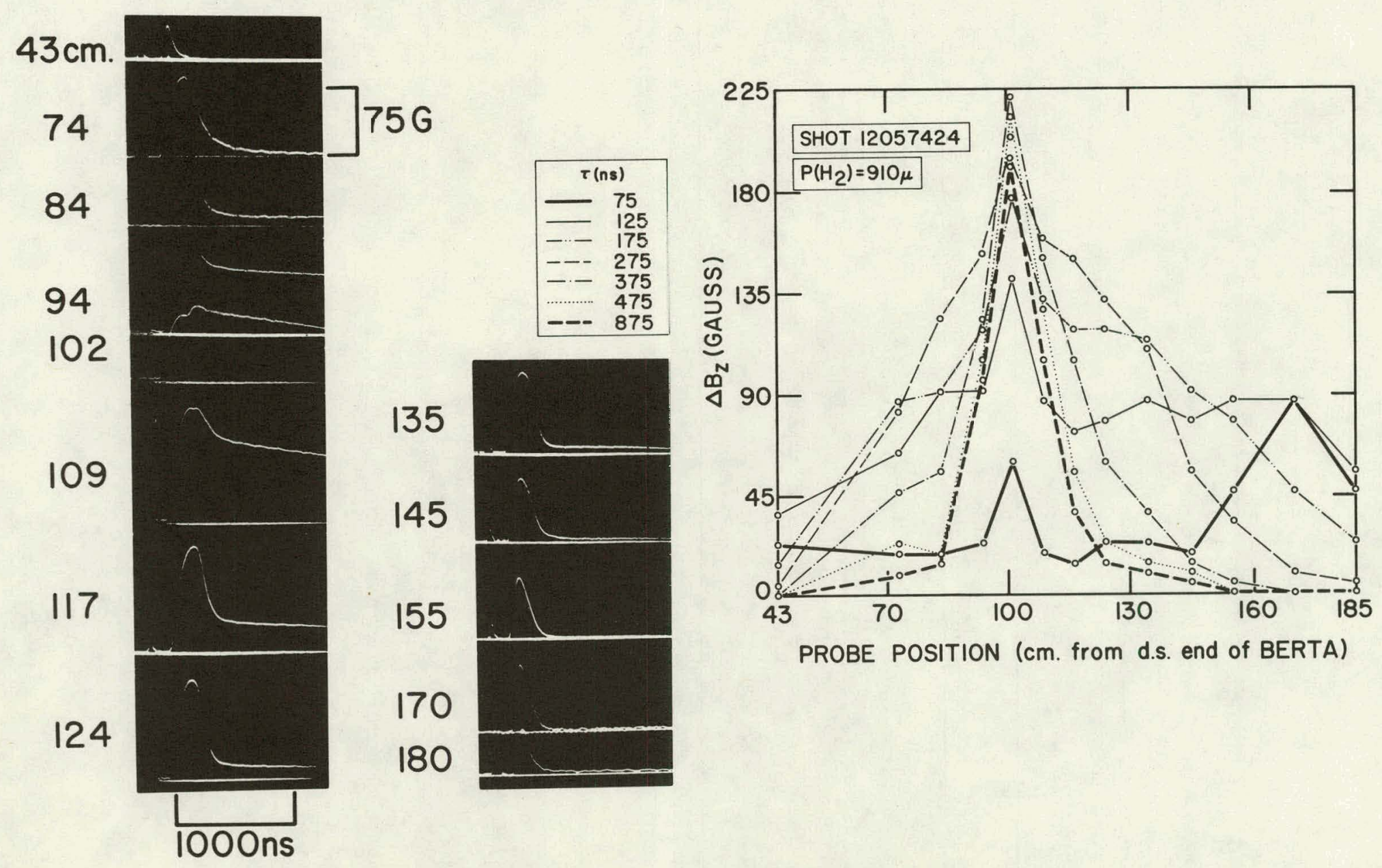


progresses, however, the axial "wings" of this uniform diamagnetic distribution are seen to decay in $300-400 \mathrm{~ns}$ to reveal a steady-state magnetic profile which defines the trapped electron ring. With the possible exception of bulk ring motion--which occasionally occurs, but is of no consequence to this discussion--the only further, consistent alterations to the magnetic profile $400-500 \mathrm{~ns}$ after injection are those describing the collisional depletion and diffusion of the circulating current which may lead to axial lengthening of the electron ring. These long-term effects occur on time scales of 10-20 $\mu \mathrm{sec}$ and have been discussed in detail elsewhere. 47

To facilitate accurate processing of the formidable amounts of data which contributed to plots such as these, Fortran IV programs were written for a Digital Equipment Corporation PDP-15/GT-40 computer equipped with peripherals which included a Scriptographics digitizing tablet for direct analysis of the oscillographs. The resolution of the tablet was $0.25 \mathrm{mra}$, well within the accuracy required for this work.

Primarily, these data-handling and display programs performed four functions: (1) the digitized points from the oscillographs were stored on magnetic tape as matrix coordinates in terms of centimeter deflections from 
timing (abscissa) and baseline (ordinate) fiducials;

(2) the proper factors were then applied to each set of data points (such as oscilloscope sweep rates, amplifier gains, oscilloscope camera reduction/enlargement factors, magnetic probe calibration factors, integrator RC-constants, etc.) to obtain the X- and Y-coordinates of each point in terms of "nanoseconds" and "Gauss", respectively; (3) a "final data" file was then created which computed, via linear interpolation between digitized points, the probe signal (in Gauss) every $100 \mathrm{~ns}$ from the timing fiducial, up to $1500 \mathrm{~ns}$; this file was then stored on tape; (4) a plotting routine was developed to output the final data files in graphic format as field profiles, displaying probe position on the horizontal axis of the graph (in terms of "centimeters from the downstream mirror") and diamagnetic field strength on the vertical axis. The field profiles were parameterized to the 100 ns time-steps, and an arbitrary number of the time-stepped profiles (up to 15) could be displayed on the same plot, giving the time history of the diamagnetic field profile. We now turn to some representative plots displaying the time-dependence of the axial diamagnetic field profiles for rings trapped in various backfill gas 
pressures. These are displayed in Figures (3-12), $(3-13),(3-14)$, and $(3-15)$, as well as Figure (3-11). These traces were selected as being typical representatives of the field of data accumulated at each pressure setting. The features these selected data illustrate were consistent in the remainder of the data, within the limits of normal shot-to-shot statistical variations.

- Overall, the salient features common to all of the diamagnetic profiles obtained in the pressure range of interest are twofold: (1) the buildup of a uniform, axial diamagnetic field distribution within 100-200 ns after the onset of diode current, and (2) the prompt relaxation of this field to reveal the steady state diamagnetic profile of the trapped electron ring. The time scale of the buildup occurs on a time scale commensurate with the beam current risetime, while the relaxation process was found to be somewhat independent of backfill pressure, ranging from 200-300 ns at 150 mTorr, to $100-150$ ns at $800-900$ mTorr hydrogen.

An interesting feature present only for those rings trapped at 150 mTorr hydrogen (this pressure having been found experimentally to be the lower gas pressure limit for proper beam charge neutralization) was the 
$-94-$

FIGURE $3-12$

Axial diamagnetic profile for the ring trapping process in 150 mTorr hydrogen. 


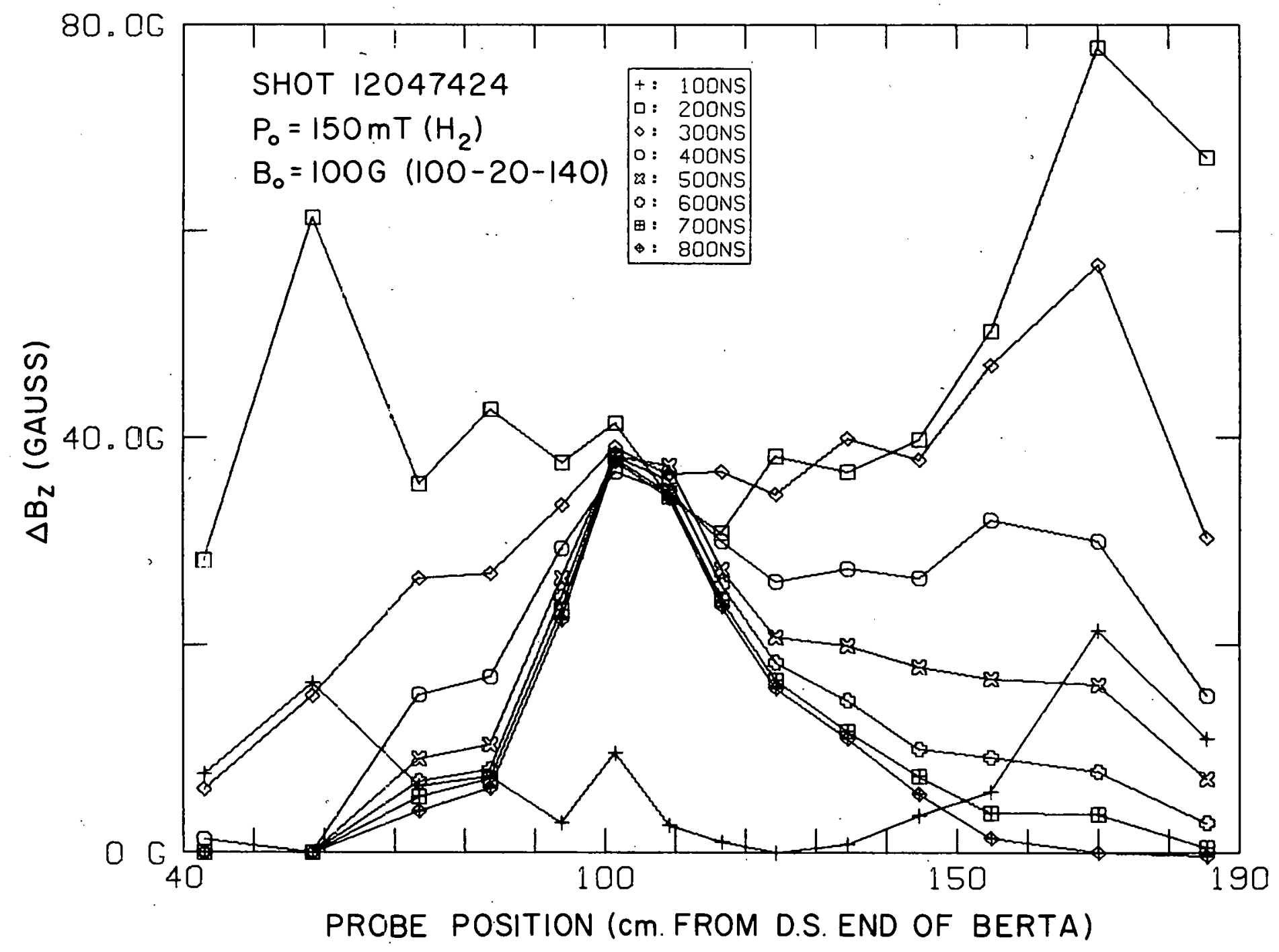


$-96-$

FIGURE $3-13$

Axial diamagnetic profile for the ring trapping process in 340 mTorr hydrogen. 


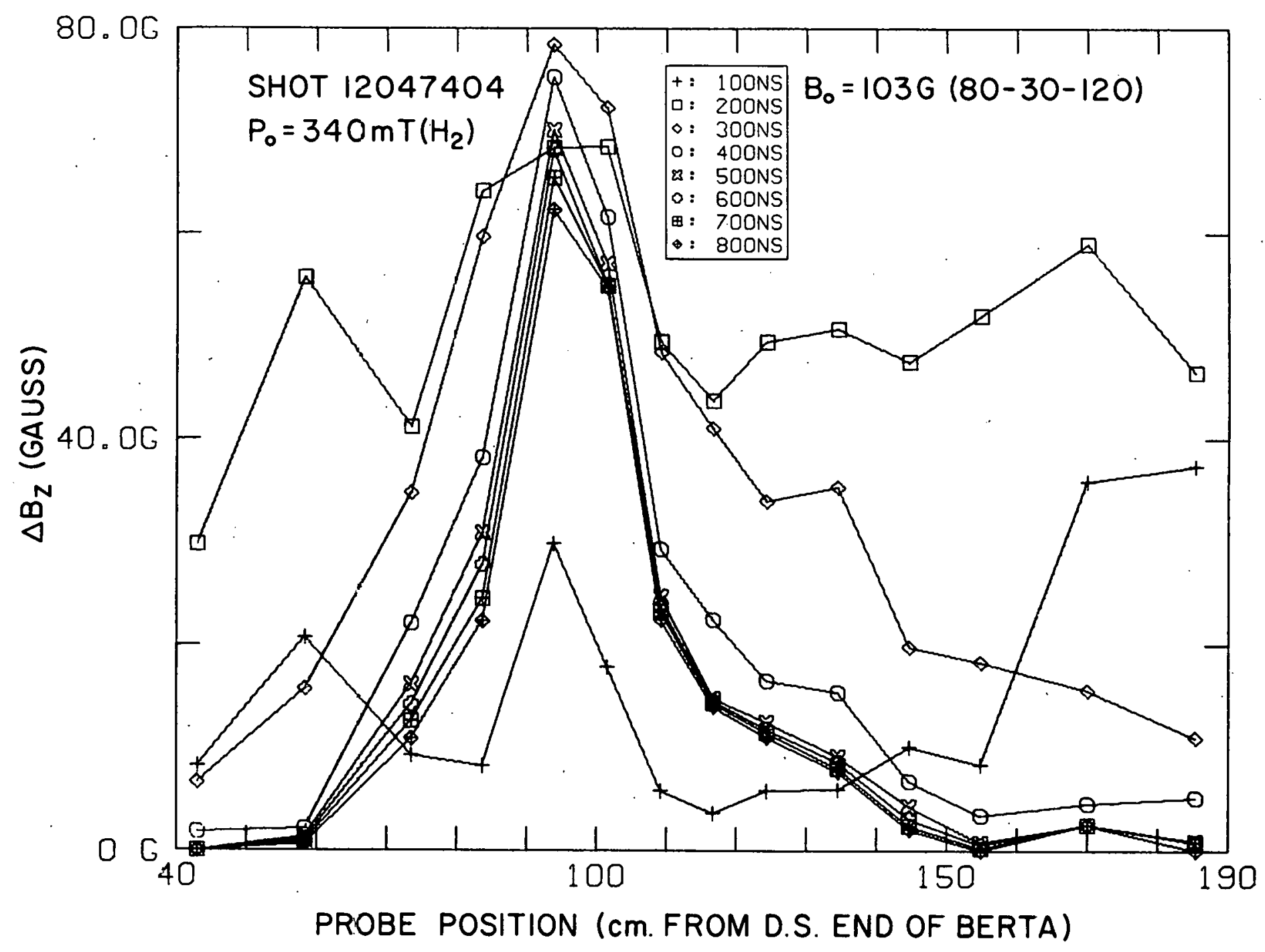


$-98-$

FIGURE $\quad 3-14$

Axial diamagnetic profile for the ring trapping process in 600 mTorr hydrogen. 


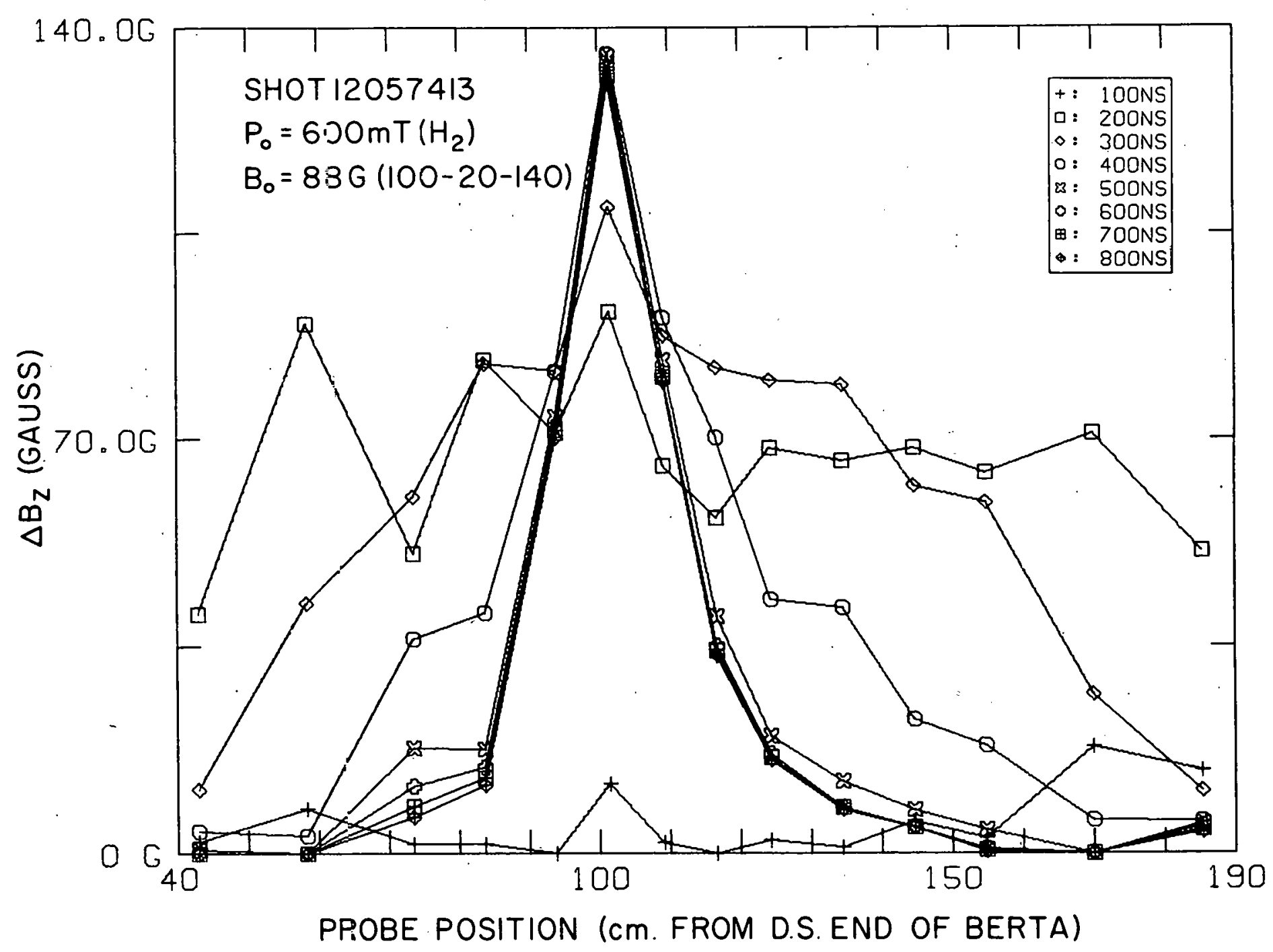


FIGURE $3-15$

Axial diamagnetic profile for the ring trapping process in $800 \mathrm{mTorr}$ hydrogen. 


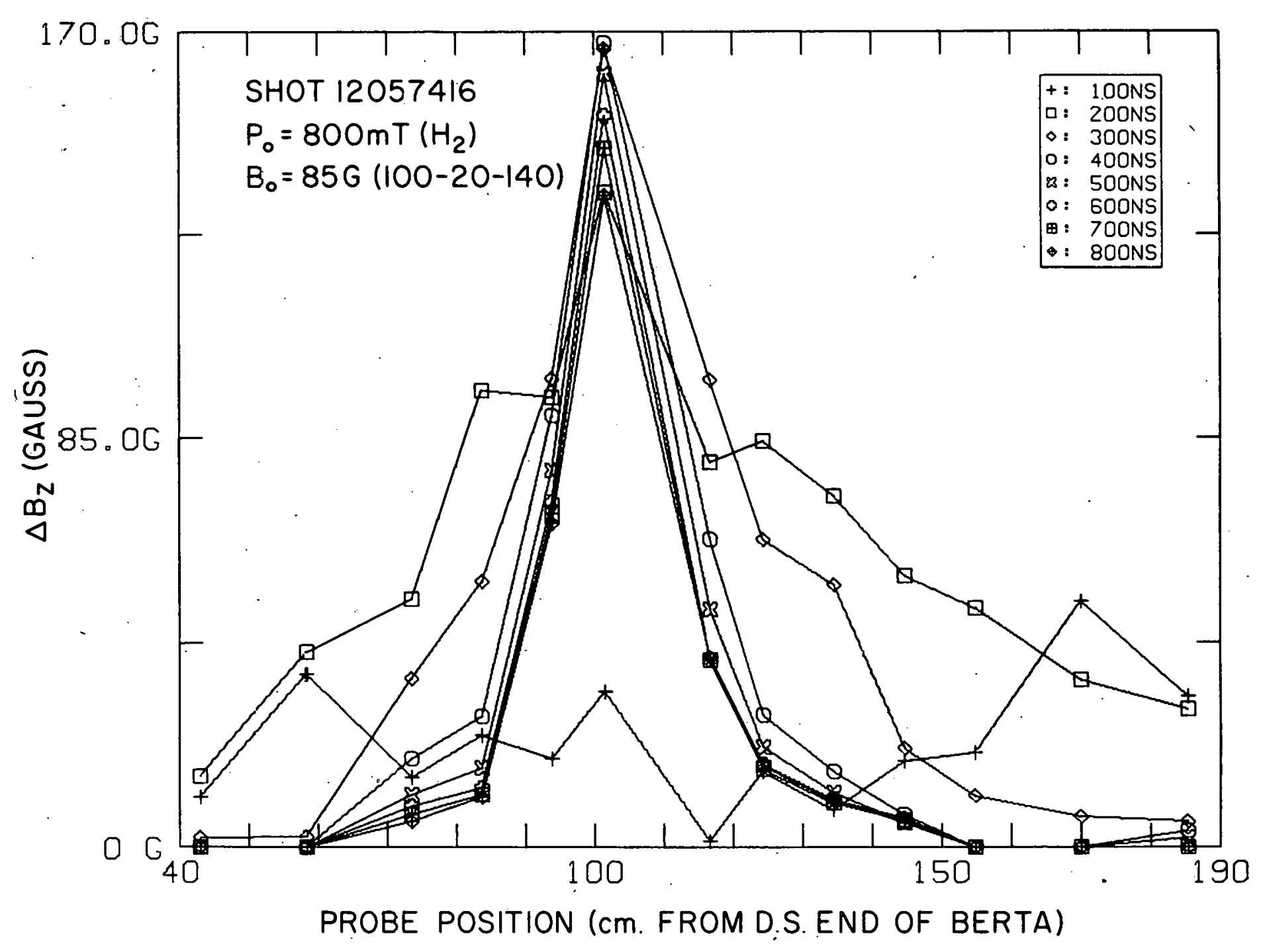

占

FIGURE 3-15 
enhanced diamagnetic field, shortly after injection, at the ends of the mirror trap. This "pile-up" effect is probably due to the beam's spending more time near the ends of the mirror trap as it spirals back and forth in the machine. A similar phenomenon was observed in the high-vacuum trapping of electron rings in the Livermore Astron device. 48

\subsubsection{High-Energy Electron Current Monitor} Measurements of the circulating fast electron current density were taken at three backfill pressures representative of the range of interest, i..e.., 150, 340 , and 850 mTorr hydrogen. For a direct comparison of the observed $\mathrm{X}$-ray signals with the diamagnetic field profiles, the wire target was consistently located at the same axial position as one of the magnetic pickup probes. A comparison between the $X$-ray signals and the comparably-located magnetic probe signals at vartous locations along Berta's axis is shown in Figure (3-16). One is first struck by the similarity between the two sets of signals. At the ends of the mirror trap, both $\mathrm{X}$-ray signals and probe signals display a solitary spike with its rapid decay to zero, while, in the central regions of the machine, this initial spike is followed by a slowly-decaying tail as the electron ring becomes 


\section{$-103-$}

\section{FIGURE $3-16$}

Direct comparison of magnetic pickup probe signals with the $\mathrm{x}$-ray detector responses for injected currents at various axial positions in Berta. 


\section{COMPARISON OF MAGNETIC PROBE AND X-RAY SIGNALS}

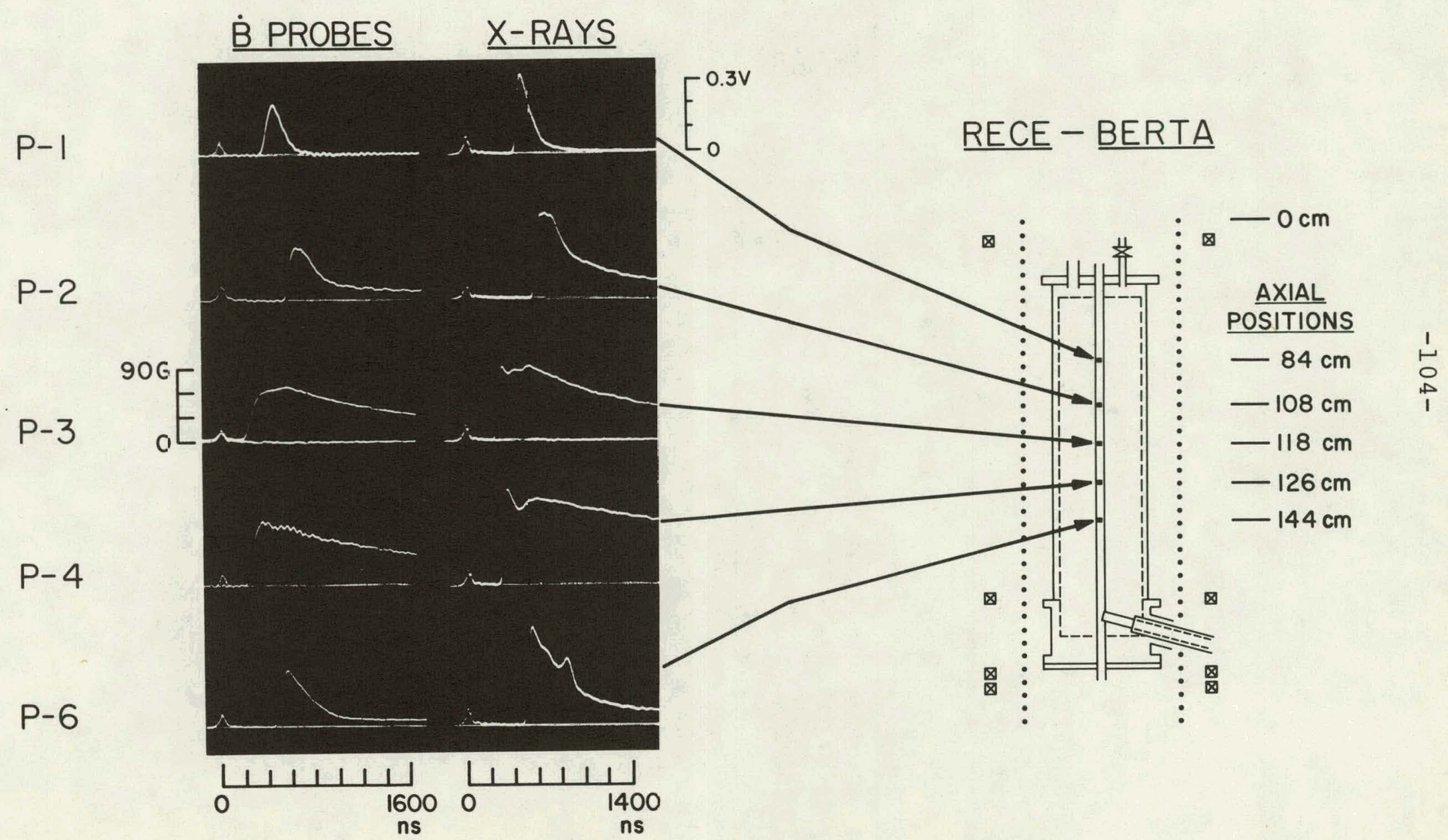


established as an entity at the bottom of the mirror well. An initial burst of $x$-radiation was consistently present during the first 100-150 ns following beam injection. This burst, which appeared whether or not the wire target was lowered into the machine, evidently resulted from either intense diode-generated $\mathrm{x}$-radiation, or a high fluence of $\mathrm{x}$-rays produced by beam primaries striking the walls of the vessel, the rod supporting the target, etc. Therefore, a series of shots was taken with the target removed from the machine (i..e.. pulled up inside the glass support rod) to obtain a background $\mathrm{x}$-ray profile as a function of time. This background profile was then subtracted from the "targetin" data.

The background $\mathrm{x}$-ray data was quite reproducible ( 158$)$ at all axial locations of the detector. The X-ray burst itself was consistently nf the same initial anplitude (about $0.2-0.3 \mathrm{~V}$ ), with a FWHM of about 100 ns, regardless of the detector's axial position. Consequently, to avoid spurious contributions to the detected signals, all $\mathrm{x}$-ray flux measurements were made at time invervals $\geq 200$ ns following beam injection (at which time the decaying "tail" from the initial burst was only 5-208--depending on the detector's axial 
location--of the total $\mathrm{x}$-ray signal).

One significant difference between the two groups of signals is the occasional presence of secondary for even tertiary) spikes on the $\mathrm{x}$-ray signals which are not observed on the magnetic field probe signals. (Note, for example, the upstream X-ray signal at $144 \mathrm{~cm}$ in Figure (3-16).) This can be directly attributed to the successive "bounces" of the electron beam spiralling back and forth in the mirror trap. (Two bounces may be discerned in the photograph of Figure (3-16).) Evidently, the plasma created by the beam on its first pass through the machine magnetically shields the probes from the secondary field pulses as the beam makes successive transits of the machine. The beam's "bounce period", as determined from traces such as the $144 \mathrm{~cm} x$-ray signal of Figure $(3-16)$, is about 120 ns. (Thus, presuming a round-trip distance of $-300 \mathrm{~cm}$, one may make a rough estimate of the axial propagation speed of the beam front as being of the order of $300 \mathrm{~cm} / 1.2 \times 10^{-7} \mathrm{sec} \sim 0.1 \mathrm{c}$.)

A direct comparison between the diamagnetic field profiles and the circulating current distribution as measured by the $\mathrm{x}$-rays is shown in Figures (3-17), (3-18), and (3-19) for the three backfill pressures studied. Since only one $x$-ray target and detector was available, 
FIGURE $3-17$

Comparison of axial diamagnetic field profiles with circulating current profiles as a function of time for the ring trapping process in 150 mTorr hydrogen. 


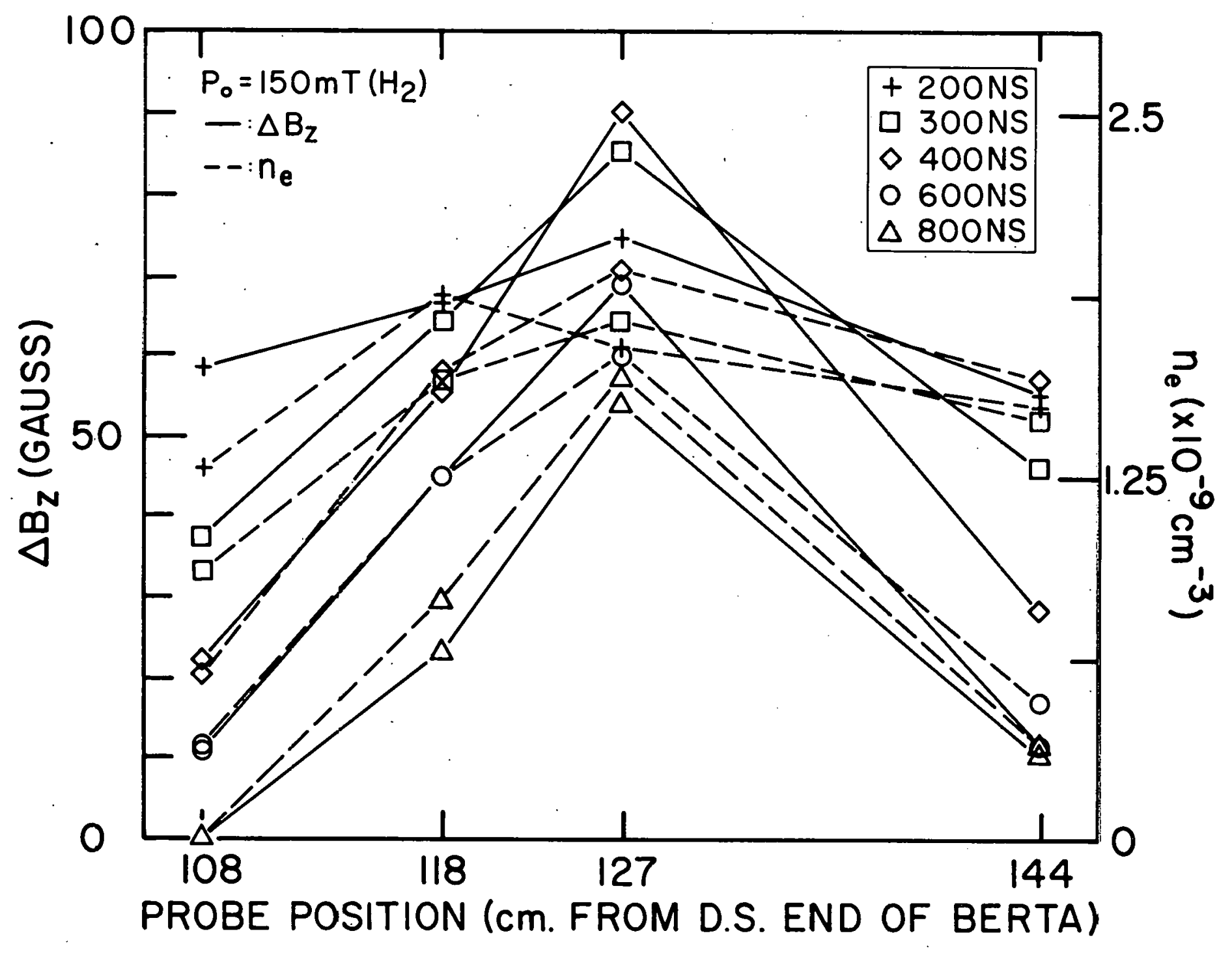


FIGURE $3-18$

Comparison of axial diamagnetic field profiles with circulating current profiles as a function of time for the ring trapping process in 340 mTorr hydrogen. 


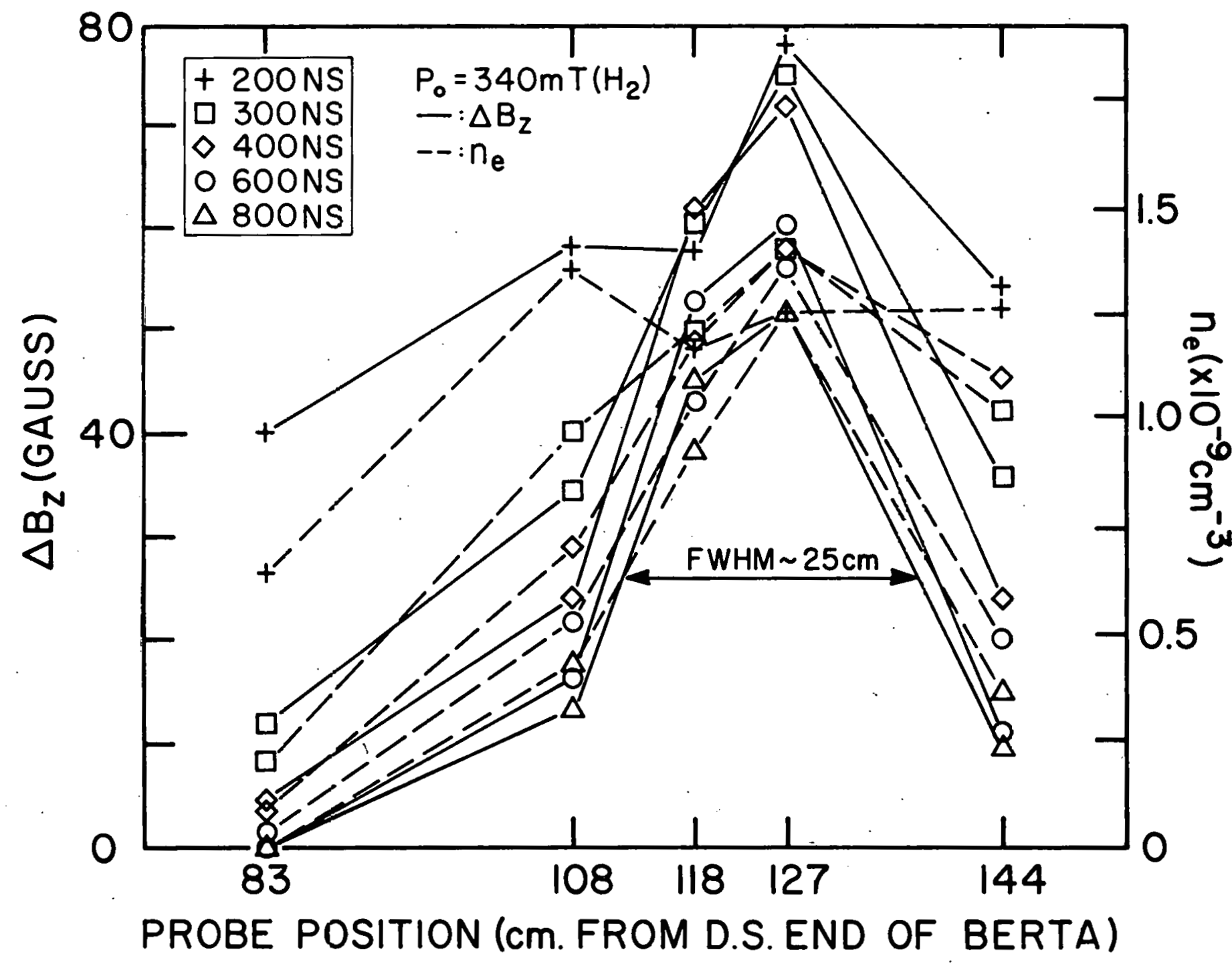




\section{FIGURE $3-19$}

Comparison of axial diamagnetic field profiles with circulating currēnt profiles as a function of time for the ring trapping process in 850 mTorr hydrogen. 


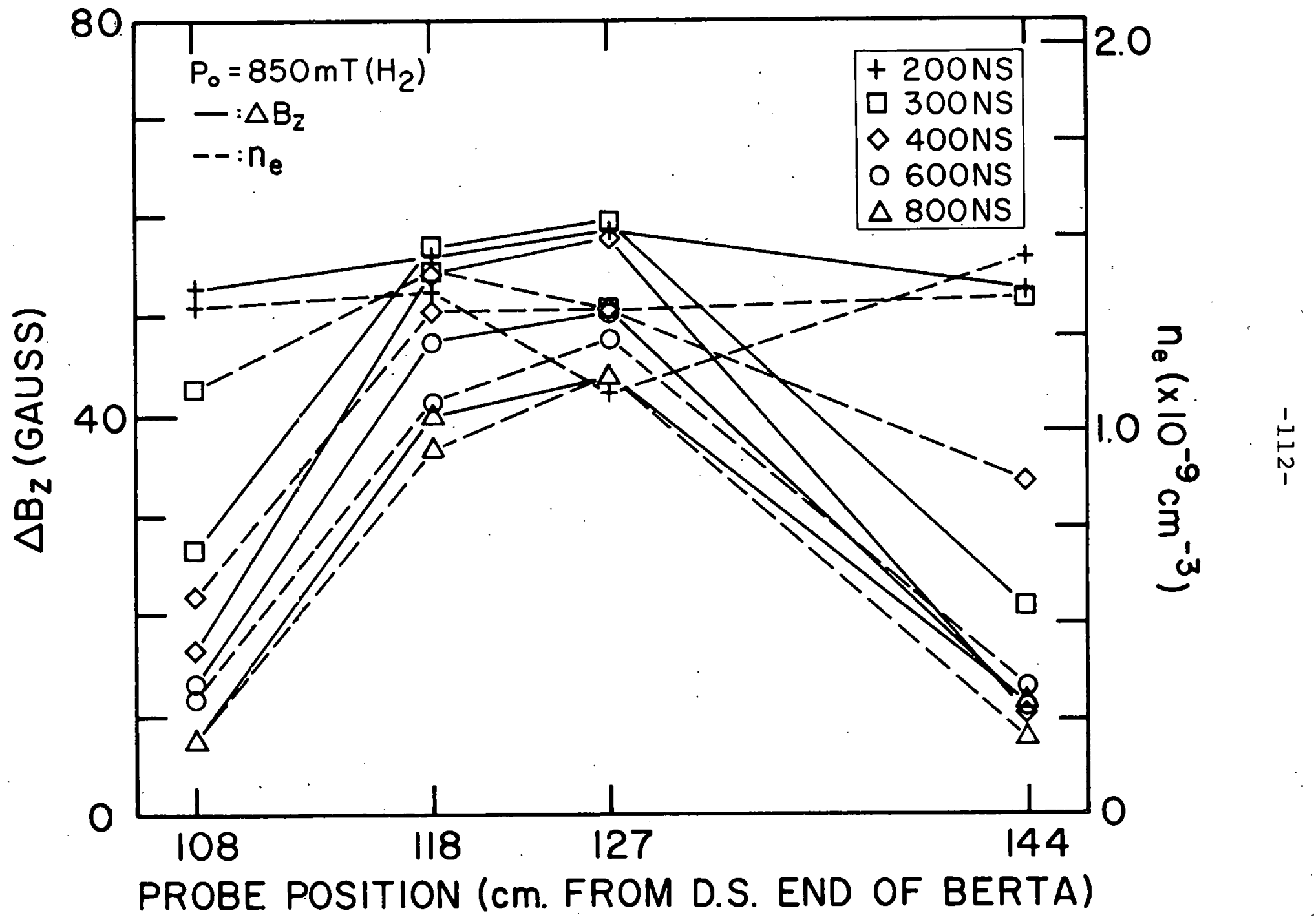

FIGURE 3-19 
the "rings" shown in Figures (3-17) - (3-19) are actually composite profiles made from representative data taken at each axial position and at each of the tank pressures of interest. For ease of relative comparisons, the $x$-ray signals and magnetic probe signals have been normalized to the same position on the plot at the center of the electron ring at one microsecond after injection. At this point in time, all plasma currents have died away, the ring has usually ceased further axial excursions, all circulating currents of high energy particles which are not magnetically-trapped electrons are no longer circulating around inside the machine, and the direct dependence of the magnetic field on the magnitude of the circulating current is a good assumption.

The above-noted similarity between the fast electron current distribution and the diamagnetic profiles is evident at all. three tank backfill pressures. In all cases, both the current and diamagnetic field distributions are quite uniform at times immediately following (100-200 ns) the injection of the electron beam. Subsequently, both the diamagnetic field and the circulating fast electron current axial distributions "decay" to form "steady-state" profiles which are very 
similar in shape. The decay rates of both the $\mathrm{x}$-ray and probe signals is seen to be slightly faster at higher backfill pressures than at lower pressures, in agreement with observations made earlier on the diamagnetic field profiles. The details of the decay rates, however, cannot be discerned given the shot-to-shot variations in the measurements.

It should be noted that the $0.2 \mathrm{~mm}$ diameter wire "killed" the electron rings over time scales of the order of 3-5 usec, depending upon the location of the target. Since the times of observation here are of the order of $500 \mathrm{~ns}$, the effects taking place during this first half microsecond of ring "life" are much faster than the long-term deleterious effect of the target's presence, and the two sets of phenomena are adequately decoupled. As a cross-check on the observed similarity between the electron current and ring magnetic field profiles, use may be made of a computation of the current distribution FWHM based on the measured FWHM of the magnetic field distribution. ${ }^{49}$ This computation includes the effects on the field brought about by image currents induced in the conducting liners of the machine, located at a radius of $23 \mathrm{~cm}$ from the machine axis. For example, for the rings trapped in 340 mTorr hydrogen, the 
calculation predicts a current FWHM of $22 \mathrm{~cm}$, using a cosine ${ }^{2}$ fit to the axial current distribution, and using the experimentally-measured FWHM of the magnetic field profile of $25 \mathrm{~cm}$. This value compares favorably with the experimentally obtained value of $25 \mathrm{~cm}$ for the current distribution FWHM, as shown in Figure (3-18).

\subsection{Interpretation of the Experimental Results}

It is not the intent of this discussion to present a full theoretical treatment of the beam primary electron current, beam/plasma, and transient magnetic field interactions. That problem is highly complex, requiring a fully self-consistent treatment of all induced (collisional) plasma and wall currents in a finite, cylindrical geometry, beam primary and trapped-ring particle loss mechanisms, and the dynamics of the transition from an extended E-layer to the axially-compacted electron ring configuration.

Instead, first-order physical arguments are presented providing one possible explanation for the trapping mechanism. These arguments are designed to establish physical limits to the processes which could account for ring trapping. As shall be shown, these limiting effects--granted the simplifications involved--lead to 
predictions consistent with the experimental observations.

\subsubsection{A Physical Model for the Trapping Process}

From the experimental results, the phenomenology of the trapping process of the electron beam in a neutral gas backfill can be summarized in the following way. For a period of 100-200 ns immediately following the injection of the electron beam into the mirror well, a non-equilibrium, uniform distribution of circulating electron current fills the mirror trap region. As the beam wraps itself back and forth between the mirrors-like yarn on a skein--a plasma sheath is created via rapid beam ionization of the background hydrogen gasfill. This plasma, too, pretty much fills the mirror region. The primary effect of the plasma, then, is to "freeze" the strong beam self-magnetic field perturbations wrought upon the background mirror field. The fast electron current distribulion (as opposed to the plasma current distribution) then evidently begins to "relax" towards an equilibrium state, with symmetrical particle losses apparent at both ends of the mirror trap.

One way of viewing this relaxation process is that it results from the layer electrons interacting with their self-magnetic fields. The reaction of the poloidal magnetic field pressure on the electrons circulating in a 
long layer is to bring about axial compression of the layer into a ring. In other words, this focussing process results from the axial Lorentz forces of the azimuthal ring electron current, $J_{\theta}$, interacting with the radial self magnetic fields, $B_{r}$, at the axial ends of the layer/ring. Two limiting factors come into play at this point: First, the self-field distribution of the beam primary particles has been "frozen" into the beam's selfcreated plasma. Therefore, the circulating current distribution cannot relax to an equilibrium at a rate faster than the relaxation rate of its self-field distribution, i.e., until the currents induced in the plasma which are responsible for maintaining the initial fast electron field distribution die away. Consequently, what happens at this stage depends entirely upon the parameters of the background plasma created by the beam. Secondly, end-on framing photography ${ }^{50}$ indicates that our electron rings are fairly thick in radial extent $(\sim 10 \mathrm{~cm})$, radial wall losses presumably being the dominant loss mechanism. Thus, as a long current distribution attempts to focus itself axially into a more oval, or circular crosssection, it may undergo considerable radial thickening and, in the process, suffer heavy particle losses via scraping on the wall "limiter". 
For the purposes of discussing limiting processes in the trapping stage of electron rings, we consider the behaviour of the cylindrical plasma channel in the absence of the fast particles. That is, we assume here that the injected beam has established a set of initial conditions in the plasma (density, circulating current density, physical dimensions, etc.), and has then been removed altogether. of course, this is not what happens experimentally, but analysis of this physical situation will provide lower limits to the temporal phenomena observed during the ring trapping mechanism.

We begin with a description of the physical circumstances surrounding the beam-created plasma channel a short time after injection (100-200 ns after beam current initialization). Since the magnetic penetration time of the copper liners inside Berta greatly exceeds the time scale of the trapping process, we may presume that the background magnetic flux initially present within the liner is conserved during the trapping process. Hence, if, from the diamagnetic effect of the beam, the magnetic field interior to the ring (see Figure (3-20), is reduced to a value $\mathrm{B}_{12}=\mathrm{B}_{\mathrm{Oz}}-\delta_{\mathrm{B}_{1 z}}$, then the field between the ring and the wall is enhanced by an added factor, $\delta \mathrm{B}_{2 z}$, to $\mathrm{B}_{2 \mathrm{z}}=\mathrm{B}_{\mathrm{Oz}}+\delta_{\mathrm{B}_{2 z}}$ where, as discussed in Chapter 6 , 


\section{$-119-$}

FIGURE $\quad 3-20$

Configuration of beam-created plasma channel in Berta (cross-sectional view). 


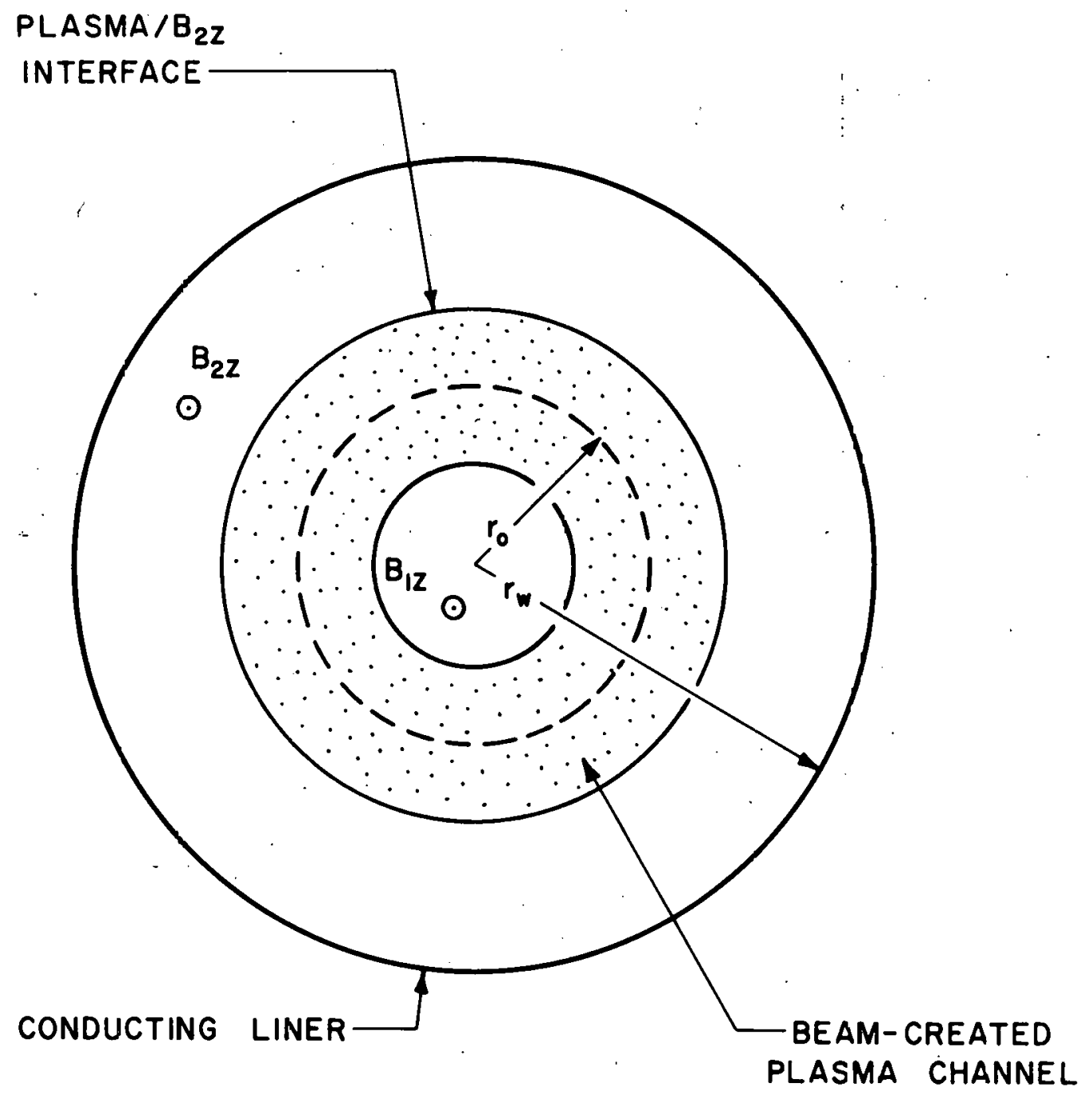




$$
\delta B_{2 z} \simeq \frac{\delta B_{12}}{\left(r_{w}^{2} / r_{0}^{2}\right)-1}
$$

(dynamic wall image currents and their contribution to the axial field have been neglected).

At the plasma $/ \mathrm{B}_{2 \mathrm{z}}$ interface, a pressure-balance equilibrium is established. At the particle level, this implies that the radial magnetic field pressure, $\mathrm{B}_{2 \mathrm{z}}^{2} / 8 \pi$, begins to push inwards on both electrons and ions in the "fluid" plasma. Due to their comparativelỳ small mass, the electrons react first to this force, moving radially inwards ahead of the ions; for the moment, we consider the ions temporarily motionless. This inward motion continues until the radial electric field formed by the space-charge separation becomes strong enough to retard further radial electron progress, and an $\vec{E}_{r} \vec{x}_{\theta}$ az1muthal drift current results.

The inductive resolve on the part of the plasma to resist changes in magnetic flux at its boundaries (or, in the event of plasma currents, within its interior) may be quantified in the following way. Given the axial orientation of the magnetic fields in the experimental geometry which are of importance to this 
discussion, Lenz's Law would therefore imply that a counter angular current, $J_{\theta}$, would be induced in the plasma to oppose any flux changes. Two independent electric fields could account for $\mathrm{J}_{\theta}$. To investigate these, we consider the conductivity tensor, $\bar{\sigma}_{\alpha}$ (MKS): ${ }^{51}$

$$
\bar{\sigma}_{\alpha}=\varepsilon_{0} \omega_{p \alpha}^{2}\left|\begin{array}{ccc}
\frac{\nu_{\alpha}}{\nu_{\alpha}^{2}+\Omega_{\alpha}^{2}} & \frac{-\Omega_{\alpha}}{\nu_{\alpha}^{2}+\Omega_{\alpha}^{2}} & 0 \\
\frac{\Omega_{\alpha}}{v_{\alpha}^{2}+\Omega_{\alpha}^{2}} & \frac{\nu_{\alpha}}{v_{\alpha}^{2}+\Omega_{\alpha}^{2}} & 0 \\
0 & 0 & \frac{1}{v_{\alpha}}
\end{array}\right|
$$

where $\alpha$ denotes the species of charged particle involved, $\nu_{\alpha}$ is the momentum transfer collision frequency, $\Omega_{\alpha}$ is the cyclotron frequency, $\Omega_{\alpha}=q_{\alpha} B_{z 0} / m_{\alpha}$, and $\omega_{p \alpha}$ is the plasma frequency, $\omega_{\alpha}^{2}=n_{\alpha} q_{\alpha}^{2} / m_{\alpha} \varepsilon_{0}$, and where we have assumed that $v_{\alpha}$ is independent of particle speed (a fairly good assumption for our experimental situation, and which shall be discussed in detail later. ${ }^{51}$,

Let us first consider the relative sizes of the terms in the conductivity tensor by calculationg $v_{\alpha}$ and $\Omega_{\alpha}$. For the ions, we shall assume that, under our experimental conditions, $\nu_{i} \gg \Omega_{i}$, postponing justification of this point until later in the discussion. 
In evaluating $\nu_{c}$, we must consider both electron-ion and electron-neutral collision frequencies. Assuming $1 \mathrm{eV}$ Maxwellian plasma electrons, the electron-neutral collision cross-section in $\mathrm{H}_{2}$ is found to be $\sim 1.5 \mathrm{x}$ $10^{-15} \mathrm{~cm}^{2}$, and is fairly constant over this energy range. 52 Thus, with a background pressure, $P_{0}$, of hydrogen,

$$
\nu_{\text {en }}=n_{o} \sigma_{e n} v_{e}-3.2 \times 10^{6} P_{o}\left(\sec ^{-1}\right)
$$

where $P_{0}$ is in units of mrorr. At 500 mTorr, $\nu_{\text {en }}$ $1.5 \times 10^{9} \mathrm{sec}^{-1}$. The electron-ion momentum-transfer cross-section is effectively the Rutherford $90^{\circ}$ scattering cross-section, $53 \sigma_{e i} \sim 2 / E(M e V){ }^{2}$ (barns), giving $\sigma_{e i} \sim 1 \times 10^{-12} \mathrm{~cm}^{2}$. Thus, with a measured plasma density $\mathrm{n}_{\mathrm{p}} \sim 1-1.5 \times 10^{12} \mathrm{~cm}^{-3}$,

$$
\nu_{e i}=n_{p} \sigma_{e i} v_{e}-9 \times 10^{7} \sec ^{-1}
$$

Therefore, in light of Equations (3.10) and (3.11), the collisional nature of the electron motion is seen to be dominated by electron-neutral collisions by at least a factor of 5 in the pressure range investigated $(150 \mathrm{mrorr}$ $<P_{0}<1000$ mTorr) : $\nu_{e} \sim \nu_{\text {en }} \cdot$

In these experiments, owing to a reduced beam energy, the base magnetic field was $B_{z 0} \sim 100 \mathrm{G}$. Hence, 
with $\Omega_{e}=1.76 \times 10^{7} \mathrm{~B}_{0}(\mathrm{rad} / \mathrm{sec})\left[\mathrm{B}_{0}\right.$ in Gauss], we have

$$
\Omega_{e} \cong 1.8 \times 10^{9}{\mathrm{rad}-\mathrm{sec}^{-1}}
$$

where we have neglected the self-fields of the plasma currents. Therefore, we see that $\Omega_{e}$ and $\nu_{e}$ are of the same order and, in general, both must be retained in the terms of the electron conductivity tensor.

With these assumptions about $\nu_{e}, \Omega_{e}, \nu_{i}$, and $\Omega_{i}$, we may write down the conductivity tensors for the plasma electrons and ions as follows (MKS):

$$
\bar{\sigma}_{e}=\varepsilon_{0} \omega_{p e}^{2}\left|\begin{array}{ccc}
\frac{\nu_{e}}{v_{e}^{2}+\Omega_{e}^{2}} & \frac{-\Omega_{e}}{v_{e}^{2}+\Omega_{e}^{2}} & 0 \\
\frac{\Omega_{e}}{v_{e}^{2}+\Omega_{e}^{2}} & \frac{\nu_{e}}{v_{e}^{2}+\Omega_{e}^{2}} & 0 \\
0 & 0 & \frac{1}{v_{e}}
\end{array}\right|\left(\nu_{e}{ }^{\left.-\Omega_{e}\right)}\right.
$$


$\bar{\sigma}_{i} \cong \frac{\varepsilon_{0} \omega_{p i}^{2}}{v_{i}}\left|\begin{array}{ccc}1 & \frac{\Omega_{i}}{v_{i}} & 0 \\ \frac{\Omega_{i}}{v_{i}} & 1 & 0 \\ 0 & 0 & 1\end{array}\right|\left(v_{i} \gg \Omega_{i}\right)$

First of all, a radial current may exist in which

$$
J_{\alpha r}=\sigma_{\alpha r r} E_{r}+\sigma_{\alpha r \theta_{\theta}} E_{\theta}
$$

That is, the radial and azimuthal electric fields could also contrive to bring about bulk, radial plasma motion. However, space-charge fields would build up so rapidly across the radial width of the plasma column as to counteract such a radial current flow almost instantaneously. Thus, $J_{r}$ is constrained to be zero, implying the necessary condition that, for all current-carrying species,

$$
E_{r} \approx-\frac{\sigma_{\alpha r \theta}}{\sigma_{\alpha r r}} E_{\theta} .
$$

For the moment, we have assumed that the ions are immobile. (That this is indeed true under the conditions of the experiments shall be shown below.) In this case, the electrons are the primary current-carriers and the supposition that $\nu_{e}-\Omega_{e}$ in Equation (3.13) leads to 


$$
-\sigma_{e r \theta} \sim \sigma_{e r r} \sim \sigma_{e \theta r} \sim \sigma_{e \theta \theta}
$$

Hence, from Equation (3.16), we would infer that

$$
E_{r} \sim E_{\theta}
$$

as a necessary requirement for $J_{r} \sim 0$ and $\nu_{e} \sim \Omega_{e}$.

We now turn to the angular counter-currents, $J_{\theta}$.

In general, the azimuthal counter-currents induced in the plasma for the $\alpha^{\text {th }}$ species will be of the form

$$
J_{\alpha \theta}=\sigma_{\alpha \theta r} E_{x_{q}}+\sigma_{\alpha \theta \theta} E_{\theta} \text {. }
$$

In light of the simplifications that $\nu_{e} \sim \Omega_{e}$ and $\nu_{i} \gg \Omega_{i}$ ' this relationship becomes

$$
\begin{array}{ll}
J_{e \theta}=\sigma_{e \theta r} E_{r}+\sigma_{e \theta \theta} E_{\theta} & \left(\nu_{e} \sim \Omega_{e}\right) \\
J_{i, \theta}=\sigma_{i \theta \theta} E_{\theta} & \left(\nu_{i} \gg \Omega_{i}\right) .
\end{array}
$$

That is, the net angular induced current will be driven by two electric fields, $E_{r}$ and $E_{\theta}:$. the radial electric field drives only electrons in an $\vec{E}_{r} \times \vec{B}_{z o}$ (Hall) drift, while the azimuthal field drives both electrons and ions "resistively" in directions parallel to the electric field. 
The net counter current density may be calculated from experimental observations. In this discussion, we shall approximate the plasma current channel in Berta immediately following injection as a long cylinder of circulating charge of length $\Delta z$, mean radius, $r_{0} ;$ and radial thickness $\Delta r$. For this geometry, the current density, $J_{\theta}$, is related to the axial magnetic field. it produces by the relationship 54

$$
J_{\theta}=\frac{\left((\Delta z)^{2}+4 r_{O}^{2}\right)^{1 / 2}}{1.26} \frac{\delta B_{I z}}{\Delta z \Delta r}\left(A / \mathrm{cm}^{2}\right)
$$

where the dimensions of $\Delta z, r_{0}$, and $\Delta r$ are in $\mathrm{cm}$, and $\delta B_{1 z}$ is expressed in Gauss. (This relationship neglects the effects of radial thickness on the dependence of $J_{\theta}$ on $B_{z}$. For rings of our radial geometry $(\Delta r \sim r)$, Woodal $1^{55}$ has shown computationally that this assumption is justified, radial thickness effects constituting less than a 10 of effect.) Presuming $\Delta z \sim 140 \mathrm{~cm}, \Delta \mathrm{r} \sim 10 \mathrm{~cm}$, and $r_{0} \sim 10 \mathrm{~cm}$, we have $J_{\theta} \sim \delta B_{1 z} / 12.6\left(A / \mathrm{cm}^{2}\right)$. From the data of Figures $(3-11)-(3-15)$, we see that a typical value of the axial diamagnetic field immediately following injection of the beam is $\delta B_{1 z}-50 \mathrm{G}$. Hence, $\mathrm{J}_{\theta}-4 \mathrm{~A} / \mathrm{cm}^{2}$. From the experimental data, we may make a good estimate of the size of $E_{\theta}$. In this model, we have a 
conducting cylinder of charge in an axial magnetic field. If an attempt is made to change the magnetic flux circumscribed by the cylinder, an electric field is induced around the cylinder to counter the flux change. BY Faraday's Law, we have

$$
\oint \vec{E} \cdot d \vec{t}=-\int_{S} \frac{\partial \vec{B}}{\partial t} \cdot d \vec{S}=\frac{-\partial \Phi}{\partial t}
$$

In our geometry, then, if the magnetic field is constant within the cylinder (a good assumption since the rotating cylinder corresponds to a single-turn solenoid), then

$$
E_{\theta}=\frac{-r_{0}}{2} \frac{\partial B_{1 z}}{\partial t}
$$

From the data in the intermediate pressure range (Figure (3-14)), the magnetic field decay rate is typically seen to be $\partial B_{1 z} / \partial t \simeq \Delta B_{1 z} / \Delta t \sim 50, G / 150 \mathrm{~ns} \sim 0.35 \mathrm{G} / \mathrm{ns}$. Thus, from Equation $(3.24)$ with $r_{0} \sim 10 \mathrm{~cm}$, we find $E_{\theta} \sim 20 \mathrm{~V} / \mathrm{cm}$. It may appeax inconsistent that values of diamagnetic field decay rates have been measured from data such as Figures $(3-11)-(3-15)$, since we assume in this discussion that no fast electrons remain in the plasma, but electron rings are clearly seen to have been trapped in those data. The justification is that these rates of change of field have been measured near the extrema of 
the trapping region (probes \#2, or \#13), which are a couple of ring diameters away from the location of the confined electron ring. Hence, the persisting ring fields do not affect these measurements noticeably.

At this point, let us consider ion mobility. From tabulated data, 56 one finds that a field of $\sim 20 \mathrm{~V} / \mathrm{cm}$ causes hydrogen ions to drift at a speed of $\sim 4 \times 10^{5}$ $\mathrm{cm} / \mathrm{sec}$ in $500 \mathrm{mTorr}$ hydrogen (parent gas). So, on the time scale of ring trapping $(0.5 \mu \mathrm{sec})$, the ions can move, at most, a couple of millimeters. Therefore, for all practical purposes, the ions have been truly immobilized by collisions during the trapping process, and all currents induced in the plasma must be carried by the electrons. Hence, the assumption that $\nu_{i} \gg \Omega_{i}$ in this process is well founded.

From the values of $\nu_{e}$ and $\Omega_{e}$ calculated above, we may calculate $\sigma_{e \theta r}$, and $\sigma_{e \theta \theta}$. If we take $P_{o} \sim 500$ mrorr, then, from the microwave interferometry results, we have $n_{p} \sim 1 \times 10^{12} \mathrm{~cm}^{-3}, \nu_{e} \sim \Omega_{e} \sim 1.5 \times 10^{9} \mathrm{sec}^{-1}$, and, inserting these values into Equation (3.13), we find $\sigma_{e \theta r} \sim \sigma_{e \theta \theta} \sim 10 \mathrm{mho} / \mathrm{m}$. Thus, we may use these values, along with the values calculated for $\mathrm{E}_{\theta}$ and $J_{\theta}$ in Equation (3.20), to find $E_{r} \sim 22 \mathrm{~V} / \mathrm{cm}$. That is, to the accuracy of our claculations and measurements, 
$E_{r} \simeq E_{\theta}=20 \mathrm{~V} / \mathrm{cm}$. Therefore, the requirement that $J_{r}=0$ (i.e... (Equation (3.18)) is verified experimentally.

We may invert the conductivity tensor to obtain the resistivity tensor 57

$$
\bar{\sigma}_{e}^{-1}=\bar{n}_{e}=\frac{m_{e}}{n q^{2}}\left|\begin{array}{ccc}
\nu_{e} & \Omega_{e} & 0 \\
-\Omega_{e} & \nu_{e} & 0 \\
0 & 0 & \nu_{e}
\end{array}\right|=\left|\begin{array}{ccc}
\eta_{T} & n_{H} & 0 \\
-\eta_{H} & n_{T} & 0 \\
0 & 0 & n_{\|}
\end{array}\right| \text {. }
$$

The diagonal terms in the tensor (3.25) are evidently independent of the magnetic field. However, this is only true if the collision frequency, $\nu_{e}$, is independent of the speed of the electron. 57 It has been determined experimentally ${ }^{58}$ that the electron collision crosssection for electrons passing through hydrogen varies by only about 308 in the electronic energy range $0.1 \mathrm{eV}$ $<\mathrm{E}_{\mathrm{e}}<10 \mathrm{eV}$. Hence, for all practical purposes for our assumed plasma temperature range $\left(\mathrm{T}_{\mathrm{e}} \sim 1 \mathrm{eV}\right)$, the collision frequency is not strongly dependent on the particle speed, and it is reasonable to assume that $n_{T} \cong n_{\|}$. The restriction that $j_{r} \simeq 0$ gives 


$$
\begin{aligned}
& E_{r}=n_{r \theta} J_{\theta} \\
& E_{\theta}=n_{\theta \theta} J_{\theta} .
\end{aligned}
$$

With this elementary model of the plasma currents as a rotating cylinder of charge, the (constant) magnetic flux interior to the cylinder is very simply related to the circulating current, I, as

$$
\Phi \cong L I
$$

or, assuming a spatially-uniform current density and using Equation (3.16),

$$
\delta_{B_{1 z}} \cong \frac{L \Delta r \Delta z}{\pi r_{0}^{2}} J_{\theta}=\frac{L \Delta r \Delta z}{\pi r_{0}^{2}}\left(\sigma_{e \theta \theta}+\frac{\sigma_{e \theta r}^{2}}{\sigma_{e r r}}\right)_{\theta}
$$

where $L$ is the self-inductance of the cylinder, $r_{0}$ is its mean radius, and $\Delta r$ is its radial thickness.

Taking time derivatives of Equation (3.28) (neglecting changes in the cylinder geometry) and using Equation (3.26b) and Faraday's Law, we find

$$
J_{\theta} \cong-\frac{L \Delta r \Delta z}{2 \pi r_{0}} \frac{J_{\theta}}{n_{\perp}}
$$

the solution for which is 


$$
J_{\theta}(t)=J_{\theta_{0}} e^{-x t}+c_{,} x=\frac{2 \pi r_{0} \eta_{\perp}}{\Delta r \Delta z L}
$$

Moreover,

$$
\frac{2 \pi r_{0}{ }^{n}}{\Delta r \Delta z}=R_{\text {eff }}
$$

where $R_{\text {eff }}$ is the effective circuit resistance of the plasma. Therefore, in the absence of a driving term (i..e.., loss of primary electrons), we would expect to see the self-magnetic field from this cylindrical plasma decay with a time constant

$$
\tau_{\text {Decay }} \sim \frac{L}{R_{\text {eff }}} .
$$

If, as above, we approximate the plasma by a long, single-turn cylinder of length $\Delta z$, of mean radius $r_{0}$, and of radial thickness $\Delta r$, then the inductance of the circulating current distribution is, to a good approximation (accurate to $0.5 \%$ for $\Delta r \sim r \ll \Delta z$ ), 59

$$
I=0.8 \mathrm{Fr}_{\mathrm{O}}-0.0125 \frac{\mathrm{r}_{\mathrm{O}} \Delta \mathrm{r}}{\Delta \mathrm{z}}\left(0.693+\mathrm{B}_{\mathrm{S}}\right) \quad(\mu \mathrm{H})
$$

where $F$ and $B_{S}$ are geometric factors dependent on $2 r_{0} / \Delta z$, and $\Delta z / \Delta r$, respectively. (For long, fairly thin solenoids, $F$ is typically in the range of $0.002-0.008$, and $B_{s}$ 
is usually between 0.20 and 0.30.$)$ Presuming $r_{0} \sim \Delta r \sim 10$ $\mathrm{cm}$, and $\Delta z \sim 140 \mathrm{~cm}, F=3.3 \times 10^{-3}$ and $\mathrm{B}_{\mathrm{S}}=0.3$, giving L $\sim 1.7 \times 10^{-8}$ Henry.

(Consideration was also given to the possible effects of the copper, conducting liners inside Berta on the inductance calculation, Equation (3.33), which assumed an isolated layer of the specified geometry. A calculation was made of the inductive coupling which might exist between the ring and the liner currents and, for current layers on the order of $100 \mathrm{~cm}$ in length [as is the case here immediately following beam injection], the correction term to Equation (3.33) is only about 3\%. Hence, this coupling effect was neglected for our purposes.)

$$
\text { Since } n_{T}=n_{\|} \text {, we may re-express Equation (3.31) }
$$

as

$$
R_{e f f}=\frac{2 \pi r_{0}}{\Delta r \Delta z} \frac{m_{e}{ }_{e}}{n_{e} q_{e}^{2}} \cong\left(7.2 \times 10^{-2}\right) \frac{r_{o}}{\Delta r \Delta z} \frac{\Omega-c m}{\operatorname{mTorr}}
$$

Using Equations (3.33) and (3.34) in (3.32), we may then calculate the decay time constants for the induced plasma currents. Some typical results of this calculation, as well as the comparable experimentally-measured values, are shown in Table $(3-1)$. To illustrate the relative 
TABLE 3-1

Circulating Plasma Current Decay Times.

\begin{tabular}{llllll} 
Pressure & $R_{\text {eff }}$ & $L$ & L/R calc'd & $\begin{array}{l}\text { Decay } \\
\text { Times meas'd }\end{array}$ \\
\hline 150 mTorr & $0.077 \Omega$ & $17 \mathrm{nH}$ & $\sim 220 \mathrm{~ns} \sim 200-300 \mathrm{~ns}$ \\
600 mTorr & $0.31 \Omega$ & $17 \mathrm{nH}$ & $\sim 55 \mathrm{~ns} \sim 100-150 \mathrm{~ns}$ \\
900 mTorr & $0.46 \Omega$ & $17 \mathrm{nH}$ & $\sim 40 \mathrm{~ns} \sim 100-150 \mathrm{~ns}$
\end{tabular}


independence of these results on ring geometry, for $\mathrm{P}_{0}=$ 600 mTorr, a short, fat ring of dimensions $r-10 \mathrm{~cm}$, $\Delta \mathrm{r} \sim 10 \mathrm{~cm}, \Delta \mathrm{z} \sim 20 \mathrm{~cm}$ gives $\mathrm{L} / \mathrm{R} \sim 45 \mathrm{~ns}$ : (In general, for $\Delta z \gg r_{0} \gg \Delta r, L / R \sim r_{0} \Delta r / 150 n(\mu s)$, and for $r \sim \Delta r \sim \Delta z, L / R \sim(0.05) r_{0} \Delta r \Delta z /\left(6 r_{0}+9 \Delta z+10 \Delta r\right) \eta$ (us), with all dimensions in centimeters and the resistivity in $\Omega$-cm.)

Overall, the agreement between the calculated and measured field relaxation times is fairly good at low pressures. However, at higher pressures (600-900 mTorr), the calculated relaxation rates are about 2-3 times faster than the observed rates. In light of the assumptions about the idealized, rectangular cross-section of the plasma currents, however, as well as the absolute uncertainty in the collision, frequency, an overall error of a factor of $1.5-2$ is probably not unreasonable for the calculated $L / R$ times.

\subsubsection{Energy Deposition in the Sheath Plasma} One aspect of the trapping process which has been neglected so far in this discussion is that of plasma energetics. The particular point of interest here is to get an idea of how the magnetic field energy is dissipated as the uniform, circulating sheath of current initially established in the mirror well shrinks axially. 
To first order, one may compute the maximum amount of energy contained in the plasma sheath in the following way. Experimentally, we know that a uniform, diamagnetic field of (at most) - $100 \mathrm{G}$ is initially established over the length of the mirror well. Energetically, this represents a maximum energy density within the volume circumscribed by the current sheath of $2.5 \times 10^{16} \times$ $(0.1)^{2} \mathrm{ev} / \mathrm{cm}^{3}$, or about $2.5 \times 10^{14} \mathrm{ev} / \mathrm{cm}^{3}$. Since the end-on cross-sectional area enclosed by the current sheath is approximately $\pi \times 10^{2} \sim 300 \mathrm{~cm}^{2}$, the energy stored per unit axial length inside the sheath is about $7.5 \times 10^{16}$ $\mathrm{eV} / \mathrm{cm}$. As the magnetic field begins to collapse, this energy/unit length must be transferred to the plasma particles. Since the plasma line density was measured to be about $I \times 10^{13} \mathrm{~cm}^{-2}$, with a sheath circumference of about $60 \mathrm{~cm}$, we have an axial plasma electron/ion density of $6 \times 10^{14} \mathrm{~cm}^{-1}$. Therefore, the amount of energy which is deposited per electron/ion pair into the plasma from the collapse of the axial magnetic field must be approximately $7.5 \times 10^{16 / 6} \times 10^{14} \sim 120 \mathrm{eV} / \mathrm{elec}-$ tron-ion pair. Since the magnetic field decays on a time scale of typically about $1 / 3-1 / 2 \mu s e c$, this implies an energy deposition rate of 200-300 eV/electronion pair - $\mu$ sec into the plasma. At a plasma density 
of $1 \times 10^{12} \mathrm{~cm}^{-3}$, this energy deposition may be restated as being $\sim 2-3 \times 10^{14} \mathrm{eV} / \mathrm{cm}^{3}-\mu \mathrm{sec}$.

To consider this process in another way, one may perform a rate calculation (neglecting magnetic fields) involving an initial current density, $J_{\theta}$, an initial plasma density, $n_{0}$, and an initial neutral background density, $N_{0}$, in which account is taken of the various atomic processes which transpire as a result of ionization, recombination, and radiation as the current flows in the plasma/neutral gas mixture. Dr. Dave Hammer of the Naval Research Laboratory has developed a code (unpublished) involving the rate equations for just such a situation for application to beam-plasma return-current heating synthesis. 60 The code monitors plasma density, electron and ion temperatures, steadystate plasma energy density, and energy density loss rates via ionization and line radiation from the plasma. Also, plasma production via collisional ionization as well as losses due to two- and three-body recombination are included. Both the current and plasma are assumed to be infinite and homogeneous in extent.

To reproduce our experimental conditions, the following initial parameters were used in the code 
calculation: $n_{0}=2.7 \times 10^{16} \mathrm{~cm}^{-3}$ (corresponding to 400 mTorr $\mathrm{H}_{2}$ ), $\mathrm{J}_{\theta}=10 \mathrm{~A} / \mathrm{cm}^{2}$ (gives rise to about $100 \mathrm{G}$ axial diamagnetic field, cf. Equation (3.28)), and $T_{e} \sim T_{i} \sim 1 \mathrm{eV}$. Since the initial beam-created plasma density was not known, it was decided to test several different values for $n_{0}$ to see how sensitive the final plasma parameters were towards variation of this initial plasma density. Values of $n_{0}$ ranging from $2 \times 10^{10} \mathrm{~cm}^{-3}$ to $2 \times 10^{12} \mathrm{~cm}^{-3}$ were tried, with the result that the final plasma characteristics changed by only 10-20\%. The results of the calculation were that the plasma density rose quickly (within 50-100 ns) to $3 \times 10^{12} \mathrm{~cm}^{-3}$, where it remained at virtually a constant level (within 5\%). Both electron and ion temperatures remained close to $1 \mathrm{eV}$, with $\mathrm{T}_{\mathrm{e}}=1.1 \mathrm{eV}, \mathrm{T}_{i}=1.7 \mathrm{eV}$. The energy content of the plasma was found to rise rapidly (within $100 \mathrm{~ns}$ ) to about $9 \times 10^{13} \mathrm{ev} / \mathrm{cm}^{3}$, or, given the above plasma density, to an energy/electron-ion pair of about $30 \mathrm{ev}$. Finally, the energy loss rate due to line radiation was found to be $2-4 \times 10^{14} \mathrm{ev} / \mathrm{cm}^{3}-\mu \mathrm{sec}$. The plasma density, the line radiation loss rates, and the energy density of the plasma were found to scale roughly with initial plasma current density, which was examined in the range of 5 to $20 \mathrm{~A} / \mathrm{cm}^{2}$ in $5 \mathrm{~A} / \mathrm{cm}^{2}$ increments. 
We therefore see good agreement between the code calculations and the "back-of-the-envelope" computation for the energy deposited per electron-pair (within a factor of 2-4). Furthermore, we find that the code also predicts a line radiation energy loss rate easily commensurate with that necessary for the plasma to dispose of the field-deposited energy density of $\sim 9 \times 10^{13} \mathrm{ev} / \mathrm{cm}^{3}$ in a time interval of the order of a few hundreds of nanoseconds. It is also encouraging to find that the code predicts a plasma density quite close to that measured in the experimental work, $\underline{i}$.e., of the order of $1-2 \times 10^{12} \mathrm{~cm}^{-3}$.

\subsection{Summary and Discussion}

In the pressure range studied, 150 mTorr $<\mathrm{P}_{0}<910$ mTorr, an experimental handle was available for varying $\nu_{e}$ relative to $\Omega_{e}, \Omega_{e}$ remaining constant. At 150 mTorr hydrogen, $v_{e} \sim 4.8 \times 10^{8} \mathrm{sec}^{-1} \sim \Omega_{e} / 3.5$, and at 910 mTorr hydrogen, $v_{e} \sim 3 \times 10^{9} \mathrm{sec}^{-1} \sim 2 \Omega_{e}$. Hence, going back to the conductivity tensor, we could examine the cases in the range of $\sigma_{e \theta \theta} / 2<\sigma_{e \theta r}<3.5 \sigma_{e \theta \theta}$. That is, at low pressures, the Hall conductivity is almost four times better than the Pedersen conductivity, and the induced plasma currents are primarily $\vec{E}_{r} \times \vec{B}_{z O}$ drifts. At high pressure, owing to the enhanced collision rate, the Hall conductivity is 
degraded to about half the "resistive" Pedersen conductivity, $\sigma_{e \theta \theta}$, and most of the circulating electrons do so under the direct influence of $\overrightarrow{\mathrm{E}}_{\theta}$. In either event, the relevant resistivity for inductive current decay is $n_{\theta \theta}\left(n_{\perp}\right) \cdot$

Physically, this range of variation leads to the following explanation of the results which were summarized in Table 3-1:

(1) At high gas pressures, the plasma $L / R$ times were calculated to be about 40 ns (at 900 mTorr hydrogen). Hence, induced plasma currents should die away quite rapidly and any residual field decay should be due mainly to the relaxation of the fast electron current distribution. Inspection of Figures $(3-11)$ and $(3-15)$ illustrate this point, with field decays at either end of the mirror trap of $100-150 \mathrm{~ns}$.

(2) At low gas pressures ( 150 mTorr), the $L / R$ times for the plasma currents were computed to be about $250 \mathrm{~ns}$. One of three results could have been obtained at this decreased density: (1) were the observed diamagnetic field decay times shorter than the calculated 
value of $250 \mathrm{~ns}$, some fundamental physics in the above calculations has been omitted since, as mentioned in section 3.4.1, the calculated $L / R$ times are lower limits on the decay times; (2) were the observed decay times much longer than $250 \mathrm{~ns}$, the relaxation time of the high energy electron current distribution towards equilibrium greatly exceeds the decay time of the inductive plasma currents (barring the unexplainable existence of very high plasma electron temperatures), and no assumptions could be made about the limiting role of plasma current decays in the relaxation process; or (3) the observed field decay time is about 200-300 ns, which is the case as shown in Figure (3-12). In this event, the following interpretation may be offered: As discussed at the beginning of section 3.4.1, the axial compression of the E-layer into a ring cannot take place until the layer's self-fields which had been "frozen" into the plasma via induced plasma currents could relax. We have found that this process evidently involves the dynamics of only the plasma 
electron currents, and that the decay time for those currents may be explained in a simple model of the plasma sheath as an inductive cylinder with tensor resistivities. That is, the imbalance of the fast electron current distribution has driven inductive currents in the plasma which cannot relax in a time shorter than their $\mathrm{L} / \mathrm{R}$ time. At low pressures, the decay time is measured to be $250-300 \mathrm{~ns}$ and, since the field decay rates at 900 mTorr probably show negligible contributions from induced plasma current decays, inspection of the data shows the axial fields from the trapped particles to decay on a time scale $\sim 100 \mathrm{~ns}, \underline{i}$. e., faster than the plasma current decay rates observed at low pressures. Therefore, at low background densities, the axial relaxation appears to be limited by the decay rate of the induced plasma electron currents, which rates were found to be con-. sistent with the calculated values.

One question which remains is that of the detailed behaviour of the fast particles during their axial compression. The observations made in this work cannot 
easily resolve whether the dominant direction of fast electron losses are axial or radial. The "wings" of the initial fast particle distribution appear to erode from the ends inwards (cf. Figures $(3-11)-(3-12)$ ), which could be interpretated as either axial or radial losses. However, some support for axial focussing certainly exists in the trapping of the stronger E-rings, $\underline{i}$.e... at higher pressures, where $\zeta-1$ and the ring diamagnetism is seen to build up by: a factor of $\sim 2$ (ㄷ‥ Figure (3-11)) as the wings of the overall axial field profile decay.

In this context, one possible picture of the highenergy electron loss dynamics during the "relaxation" phase of the trapping process which is consistent with the observed behavior is the following: As the current distribution begins to contract axially, the internal pressure of the fast particles will try to increase the ring's radial thickness, acting against the external field pressure (including both background fields as well as the ring's self-field) in the process. If, however, the walls act directly or indirectly like "limiters" in the radial direction, then, as mentioned above, circulating ring current will be lost in direct proportion to the axial shortening of the layer to a ring, 
thereby keeping the axial current/unit length at a roughly constant level or, at most, increasing it only as a small amount of radial thickening is accommodated without significant particle losses. When internal fast particle/ plasma pressure balances the $\vec{J}_{\theta} \times \vec{B}_{r}$ axial focussing forces, the axial length has reached an equilibrium value and this process stops.

The $\mathrm{X}$-ray diagnostic described in this chapter is unsuited to determine whether, in fact, fast particles were lost preferentially in the radial or axial directions in this experiment. This is due primarily to the difficulty of eliminating spurious background radiation in monitoring Bremsstrahlung from liners, etc. The radiation levels from these processes in the machine are too low to allow good counting statistics from the kind of collimation that would be required for such a detailed measurement. The dose rates are also inadequate to permit a diagnostic such as time-integrated radiography, which could reveal Bremsstrahlung "hot spots", but would still provide no information as to the spatial or temporal histories of the particles responsible for the radiation. An alternative approach might be, as mentioned in Section (3.1), to consider an array of charged particle collectors distributed about the interior of Berta. The 
difficulties of interpretation are considerable, however, owing to the inability to discriminate against fluxes from particles which continue to circulate owing to electrostatic confinement by the conducting liners, 61 as opposed to E-layer particles which are lost in the axial focussing process. In addition, there is certainly no guarantee that the particle losses, if non-adiabatic, take place in an orderly, reproducible fashion. 
CHAPTER 4

AVERAGE RING ELECTRON ENERGY MEASUREMENTS 


\section{CHAPTER 4}

\section{AVERAGE RING ELECTRON ENERGY MEASUREMENTS}

\subsection{Introduction}

As mentioned in Chapter 1, one of the primary questions left open following the work of Phelps, et al.." was the energy loss mechanism underlying the decay of the intense rings trapped in hydrogen gas backfills. The decay rates of the ring magnetic fields were found to vary linearly with the gas backfill pressure and, for rings confined in admixtures of higher- $z$ gases, these rates scaled in a manner consistent with the predictions of classical multiple scattering theory of the high-energy electrons with the background hydrogen. In addition, the measured, linear decay rates, corrected for axial lengthening of the rings into more layer-like geometries, compared favorably with the theoretical electron diffusion calculations of Humphries. 63 Although there appeared to be a strong inference that the primary energy loss mechanisms of the ring electrons would be through classical energy losses, a direct measurement of the ring electron energy was necessary in light of the apparent contrasts in existing experimental results: linear beam 
injection into preionized backgrounds inferred beam-toplasma energy transfers of up to $25 \%$ in 1 meter, 64

while runaway, high-energy electron currents are observed to exist in toroidal discharges for several milliseconds. 65 The fact that the ring lifetimes of interest ranged from 10-20 $\mu$ sec rendered the possibility of using multichannel pulse-height techniques impractical: even with a sampling rate of the order of $10 \mathrm{MH} z$, one could not hope to generate enough counts from which to obtain a meaningful spectrum taken during the entire ring lifetime, let alone a time-resolved measurement of the average ring electron energy •

Another possible technique would have been to monitor the temporal and spatial changes in the magnetic fields carefully throughout the trapping region during the ring lifetime. Then, with a knowledge of the axial and radial ring current density profiles as a function of time (a difficult measurement), one could conceivably use Ampere's Law to infer the fast electron energies. However, this procedure is somewhat impractical in light of the detailed magnetic field map which would be required, as well as the accuracy with which the current profiles would have to be measured. It would have been quite difficult to achieve the precision necessary to 
measure energy changes of the electrons, which could be as small as $5 \%$.

In view of the difficulties perceived with the two above-mentioned measurement techniques, a third scheme, multi-channel X-ray absorption spectroscopy, was explored and eventually used to make the measurement.

\subsection{The Experimental Technique}

Multi-channel $\mathrm{x}$-ray absorption spectroscopy makes use of the strong energy-dependence of the photon massattenuation coefficient, $\mu_{a} \sim z^{5} / E_{\gamma}{ }^{3.5}$, beyond the $\mathrm{K}-$ shell absorption "edge" to determine $\mathrm{x}$-ray energies $\left(E_{\gamma}\right.$ is the $\mathrm{x}$-ray energy, $\mathrm{z}$ is the atomic number of the absorber). ${ }^{66}$ If the source of the $x$-radiation is the Bremsstrahlung generated by charged particles striking a high-z target, the energy of the particles may then be obtained through the coupling between their energy and the "hardness" of the $X$-ray spectrum they produce upon striking a target. For absorbers composed of heavier elements $(z>50)$, this technique could only be applied to $\mathrm{X}$-rays in the energy range $100 \mathrm{keV} \leqslant \mathrm{E}_{\gamma} \leqslant 1000 \mathrm{keV}$ : at higher energies, the slope of $\mu_{a}$ versus $E_{\gamma}$ flattens out and, at around $5 \mathrm{MeV}$, changes sign owing to the onset of pair production, and at energies below $100 \mathrm{keV}$ the slope of $\mu_{a}$ versus $E_{\gamma}$ is no longer continuous, being 
interrupted at the various shell absorption "edges". Excellent sets of plots of $\mu_{a}$ as a function of $x$-ray energy for several elements are widely available ${ }^{67}$ and, for reference in this work, a selection of relevant graphs appears in Appendix II.

For the sake of illustration, consider the experimental setup sketched in Figure (4-1). Shown in the illustration are two detectors located at the end of a long collimator, in front of which have been placed two absorbers of different thicknesses, $\delta_{1}$ and $\delta_{2} \cdot A$ flux of monoenergetic $X$-radiation of energy $E_{O}$ and intensity $I_{0}$ irradiates the front surfaces of the absorbers. If a calibration has been performed between the two detectors such that, for equal thicknesses of absorber, $I_{2}=k I_{1}$, then the energy of the $\mathrm{x}$-radiation is given as

$$
E_{0}=\mu^{-1}\left(\frac{1}{\delta_{2}-\delta_{1}} \ln \left(k I_{1} / I_{2}\right)\right)
$$

The dependence upon the signal ratio, $\mathrm{kI}_{1} / \mathrm{I}_{2}$, as opposed to absolute intensities of the detector signals, is greatly preferred from an experimental point of view because of possible shot-to-shot fluctuations in the $\mathrm{x}$-ray intensity striking the absorbers. 
FIGURE 4-1

Ideal absorption spectroscopy experiment. 


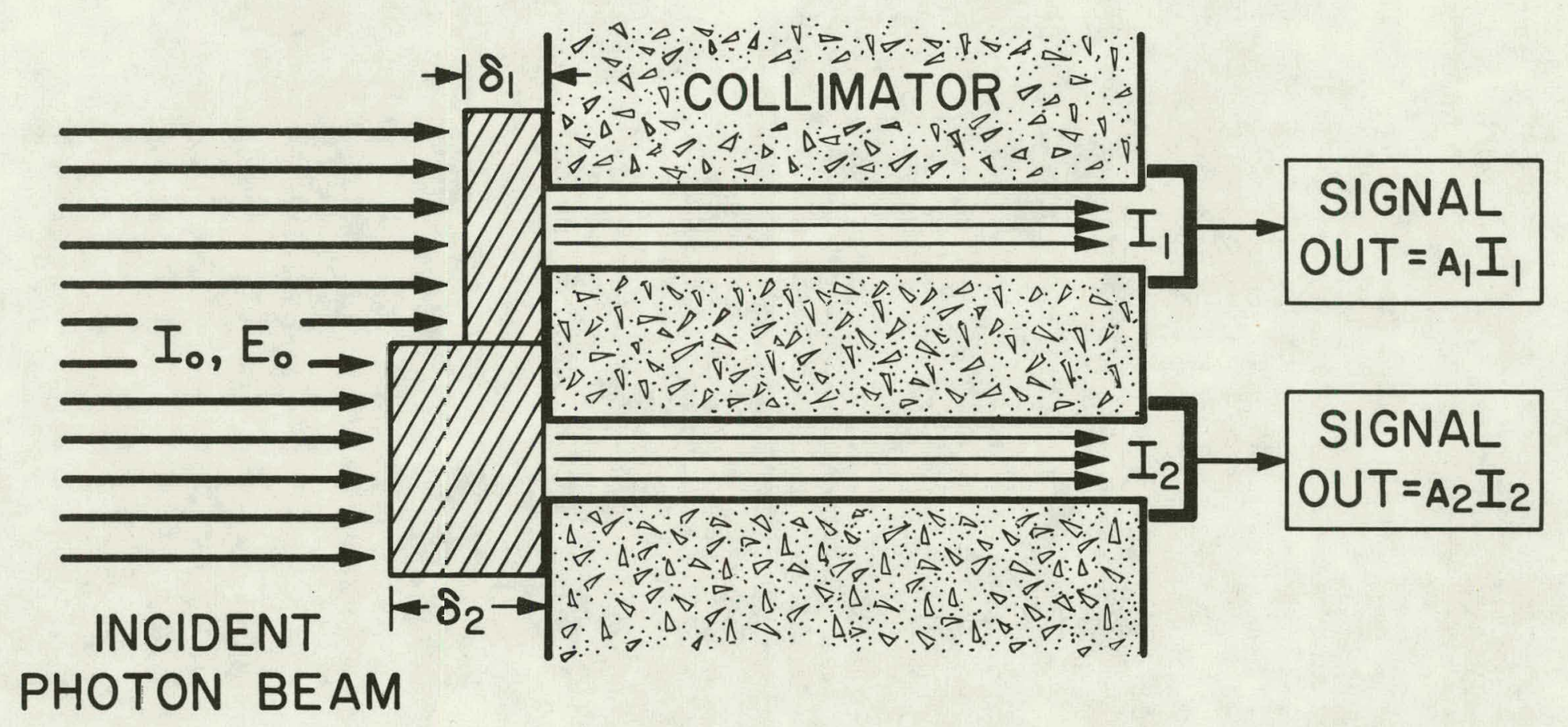

$$
\begin{array}{ll}
I_{1}=A_{1} I_{0} e^{-\mu\left(E_{0}\right) \delta_{1}} & \mu\left(E_{0}\right)=\frac{1}{\delta_{2}-\delta_{1}} \ln \left(k I_{1} / I_{2}\right) \\
I_{2}=A_{2} I_{0} e^{-\mu\left(E_{0}\right) \delta_{2}} & E_{0}=\mu^{-1}\left(\frac{1}{\delta_{2}-\delta_{1}} \ln \left(k I_{1} / I_{2}\right)\right)
\end{array}
$$

DETECTOR RELATIVE CALIBRATION FACTOR: $k=A_{2} / A_{1}$ 
The ideas expressed in the above scheme may be put to use for our purposes in the manner illustrated in Figure $(4-2)$. Shown in the sketch is the electron ring confined between the two mirror windings in the solenoidal field of RECE-Berta. The source of the $\mathrm{X}$-radiation is the Bremsstrahlung generated as the high energy ring electrons strike a small diameter filament of high-z material suspended radially across the machine. The wire would be of small enough diameter to allow for a minimal perturbation of the ring, yet large enough, and with a high enough atomic number, to take advantage of the $z^{2}$-dependence of the Bremsstrahlung cross-sections (thick target Bremsstrahlung intensity is proportional to z) ${ }^{68}$ to produce $\mathrm{X}$-ray fluxes adequate for statistically accurate measurements. A portion of the Bremsstrahlung is then monitored by a series of collimated detectors placed off to the side of the machine, each detector having had a different thickness of a high-z absorber placed in front of its respective collimator aperture. Thus, the measured quotients of the various detector signals may be used to unfold the average--and unique-$x$-ray energy and, hence, the average ring electron energy •

Several complicating factors influence the 


$$
-154-
$$

\section{FIGURE $\quad 4-2$}

Conceptual illustration of energy measurement diagnostic. 
CONCEPTUAL VIEW OF APPARATUS

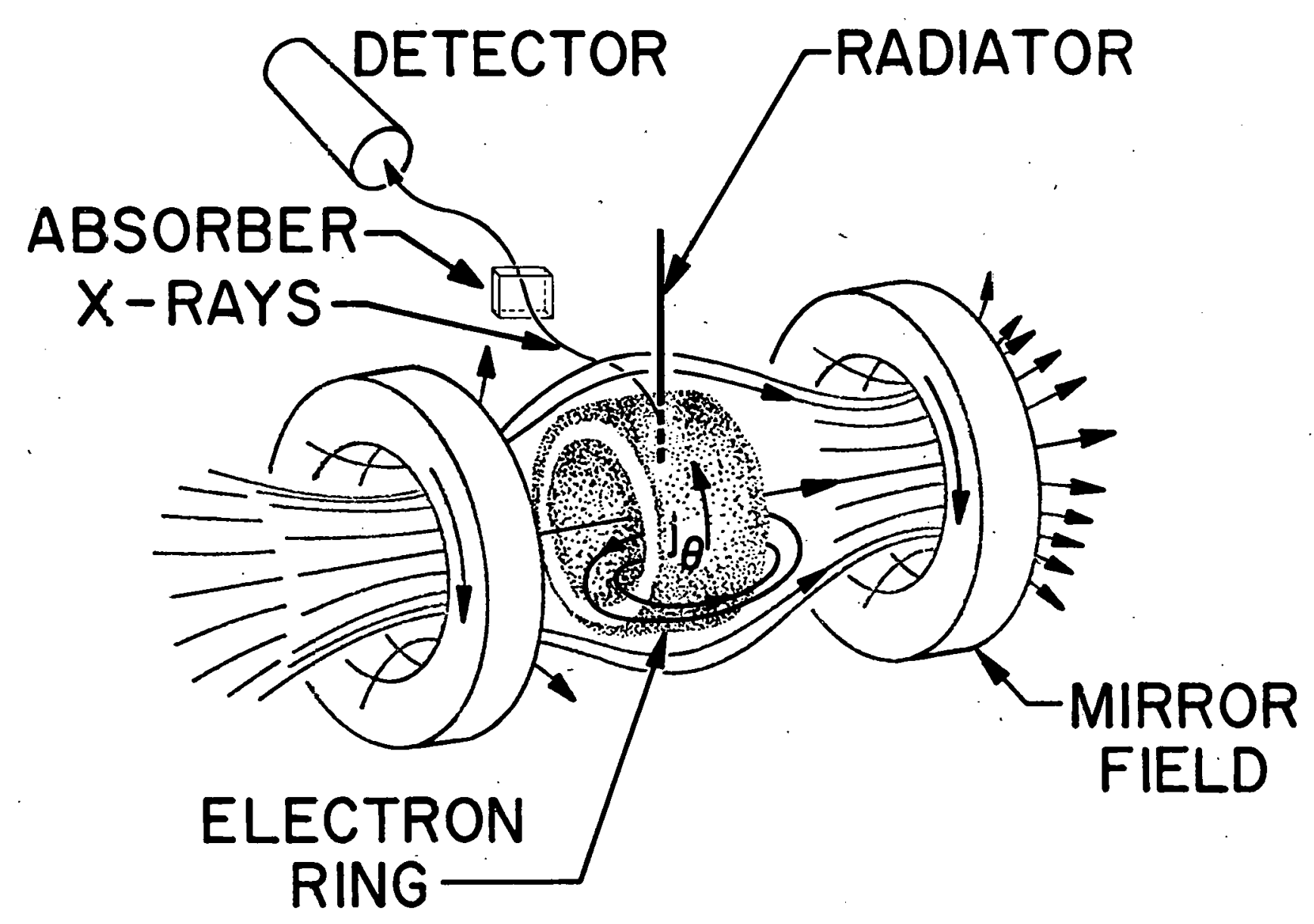


experimental practicality of the measurement. First, it is not at all clear that the ring electrons are monoenergetic. Second, even if the ring electrons were monoenergetic, the radiation produced on their striking the high-z target would consist of a spectral distribution of Bremsstrahlung up to, and including, the beam primary energy. Also, the nature of the radiation spectrum depends on the geometry of the radiator as well as its thickness and physical composition. Next, the question of the angular dependence of the Bremsstrahlung spectrum arises: presumably the detectors are of finite size and must subtend some solid angle with respect to the radiator. Thus, the differences in the radiation spectrum at different detector locations may become important. Furthermore, one must consider how the radiation spectrum is "softened"--as well as being attenuated--as the radiation passes through the various absorbing materials in the apparatus (such as the vacuum vessel walls, copper liners, Faraday shields, etc., in addition to the high-z absorbers) before interacting with the detectors. Finally, the finite size of the detector crystals makes the detection efficiency strongly dependent upon the energy of the incident radiation. The discussion of these--and other--complications 
in this measurement, and the manner in which they were handled in this set of experiments, is the subject of the next several sections of this chapter.

\subsection{Experimental Arrangement}

Top and side views of the apparatus configuration used in this measurement are shown in Figure (4-3). The tungsten wire target was hung inside the Berta tank at the minimum of the magnetic mirror well. The three collimated $x$-ray detectors were located off to the side of the Berta device. The axial location and diamagnetic "strength" of the electron rings were monitored by three "slow" magnetic probes (see section 3.2.2) located on the axis of the machine and spaced symmetrically apart by $6 \mathrm{~cm}$ about the rings' axial center.

\subsubsection{Operating Parameters of RECE-Berta}

Throughout this set of measurements, the RECEBerta facility was operated in a standard mode (cff. Chapter 2), with a mirror field of $(80-20-120), \mathrm{B}_{\mathrm{O}}=$ 190 G. Nominally, the electron beam voltage was - $500 \mathrm{keV}$, with 12-15 kA currents injected into the drift tube. The main tank pressures were 420 and 750 mTorr hydrogen, with admixtures of argon also studied later in the work. Typically, the electron rings were 
FIGURE $\quad 4-3$

Layout of the energy measurement diagnostic. 

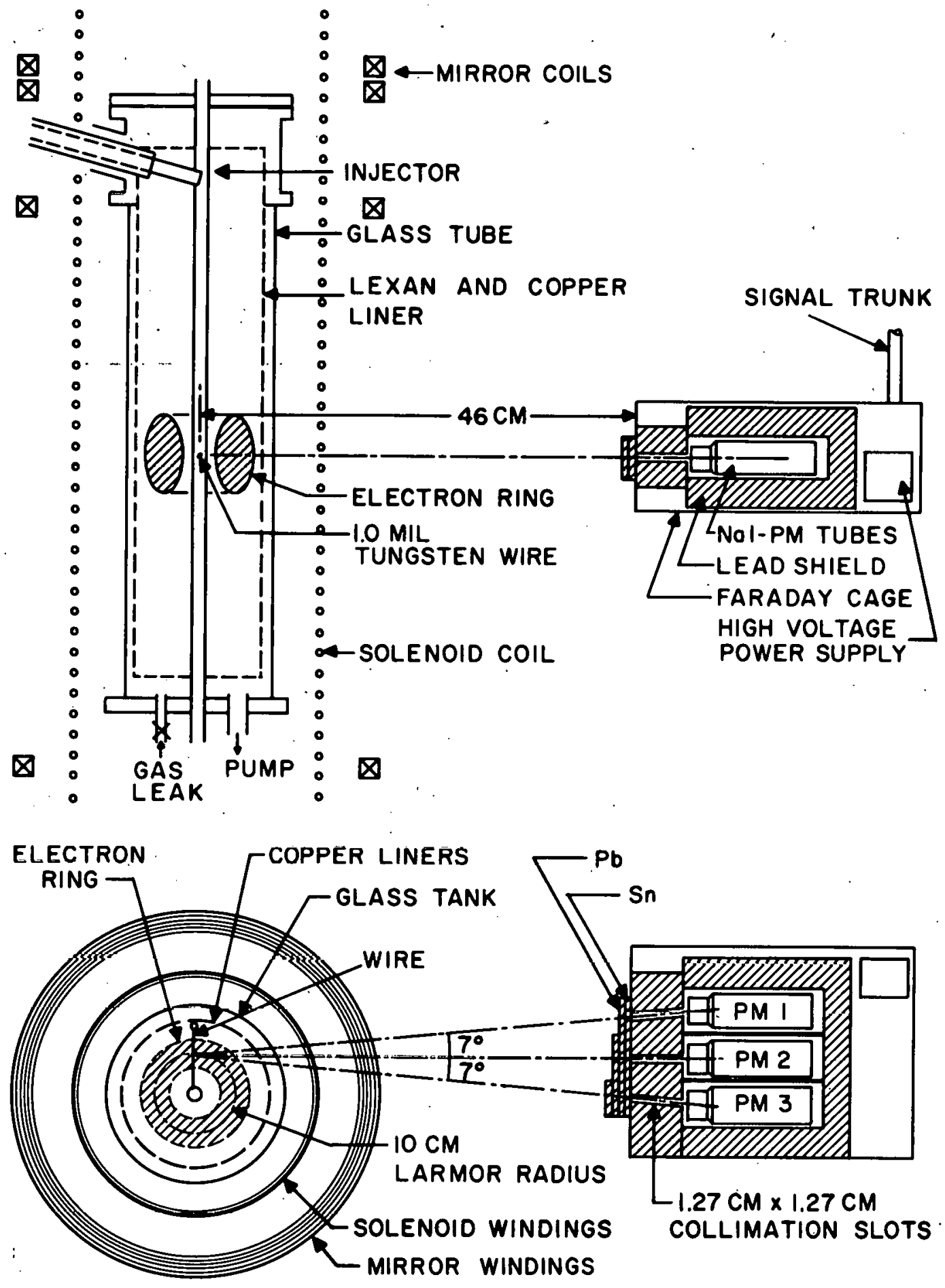
confined at the expected minimum of the magnetic well, about $110 \mathrm{~cm}$ from the downstream end of Berta (cf. Figure $(2-3))$.

\subsubsection{Bremsstrahlung Source Target}

The wire target, or radiator, consisted of a $0.025 \mathrm{~mm}$ diameter, $20 \mathrm{~cm}$ long tungsten $(z=74)$ filament with a small lead weight attached to its end. The target was suspended radially from the top of the vacuum vessel to the axis of Berta from the end of a glass rod. The rod (16 mm diam. Pyrex) was cantilevered at the top of the tank from the downstream Lucite endplate through an o-ring vacuum fitting. The rod could be moved axially, and a small counterweight was attached to its other, exterior end to insure that the rod always pressed against the top of the tank and itself did not interfere with the electron ring. The tungsten filament was extremely flexible, and one could "remove" it from the machine simply by rotating the glass rod and winding the filament up around the end of the rod.

The choice of the size of the wire radiator was a trade-off between desiring a minimal perturbation of the electron ring, yet still being large enough to produce acceptably high fluxes of radiation with which to make the measurement. One may estimate roughly the wire's 
influence on the ring lifetime as follows: assuming that the ring is about $15 \mathrm{~cm}$ in axial length, with an electron gyroperiod of about 2 ns (an electron with relativistic mass factor $\gamma \sim 2$, magnetic field $190 \mathrm{G}$ ), one finds that all particles will have interacted with the wire in a time of the order of $(15 \mathrm{~cm} / 0.0025 \mathrm{~cm}) \times 2 \mathrm{~ns} \sim 12 \mu \mathrm{s}$. As shall be discussed in Section 4.4.2.2, $500 \mathrm{keV}$ electrons striking the tungsten target will be scattered through an average angle $\theta_{\text {eFWHM }} \sim 80^{\circ}$. This implies that a very sizeable fraction of the electrons striking the target are lost from the electron ring, since an $80^{\circ}$ scattering would so severely alter an electron's trajectory in the solenoid field as to render its further participation in the primary ring current impossible. On the other hand, a $500 \mathrm{keV}$ electron loses only about $58 \mathrm{keV}$, or about $12 \%$ of its initial energy, in passing through $0.025 \mathrm{~mm}$ of tungsten. ${ }^{69}$ Therefore, the particle losses on the target are due almost entirely to large-angle scattering. In fact, the electron rings were experimentally observed to "live" for 10-12 $\mu \mathrm{s}$ with the wire located near the ring's axial center. The manner in which the wire's perturbation affected the energy measurements, and the corrections necessary to account for this effect, are the subjects of discussion in section 4.6. 


\subsubsection{Multi-Channel Detector Configuration}

As shown in Figure (4-3), the Bremsstrahlung monitor consisted of a three-detector array positioned to the side of Berta, $46 \mathrm{~cm}$ from the tungsten target. The detectors used were Harshaw Chemical Company "Integral Line" units consisting of solid, cylindrical thallium-doped sodium iodide crystals (NaI(T1)) $4.5 \mathrm{~cm}$ in diameter and $5.1 \mathrm{~cm}$ in length mated to the faces of 10-stage RCA 6342A photomultiplier tubes. The entire units were sealed in an aluminum can $0.038 \mathrm{~mm}$ thick. The accelerating dynode structures of all three detectors were wrapped in magnetic shielding (" $\mu$-metal", Conetic AA foil) to isolate the photomultiplier tubes' operation from the stray magnetic fields from Berta's solenoid windings.

The detectors were located in three tunnels inside a "lead house" which provided greater than $15 \mathrm{~cm}$ of lead radiation shielding in all directions. The Bremsstrahlung produced by the wire could intersect the detectors only through three collimated ports, consisting of $1.2 \mathrm{~cm}$ $\times 1.2 \mathrm{~cm} \times 15 \mathrm{~cm}$ square-shaped slots milled in the lead. The ports were aligned with an angular separation of $7^{\circ}$ so that the three detectors "saw" the same $10 \mathrm{~cm}$ central stretch of the radiator as it hung from the top of the 
machine. The detector array and lead shielding were housed in a solid copper Faraday cage. The entire monitor was mounted on a steel carriage with rails which permitted moving the assembly away from Berta for such purposes as changing the anode foils in the diode, diode cleaning, vacuum system maintenance, etc.

Absorbers of different thicknesses were taped to the front of the collimators. The absorbers used were pure lead which had been rolled to thicknesses of 2.52 $\mathrm{g} / \mathrm{cm}^{2}(2.2 \mathrm{~mm}), 5.04 \mathrm{~g} / \mathrm{cm}^{2}(4.5 \mathrm{~mm})$, and $7.56 \mathrm{~g} / \mathrm{cm}^{2}$ $(6.7 \mathrm{~mm})$. For brevity, these will be denoted in this work as "1Pb", "2Pb", and " $3 \mathrm{~Pb} "$ in reference to the thicknesses being multiples of $2.52 \mathrm{~g} / \mathrm{cm}^{2}$. In addition, the front cover plate to the Faraday cage adds a permanent copper "absorber" $1.43 \mathrm{~g} / \mathrm{cm}^{2}(1.6 \mathrm{~mm})$ in thickness.

In addition to the lead (and copper), a tin absorber of $1.90 \mathrm{~g} / \mathrm{cm}^{2}$ was mounted permanently to the front of each collimator. Its purpose was twofold: First, as mentioned above, a number of "unintentional absorbers" interfered--of necessity--with the target Bremsstrahlung. Consisting primarily of low-z materials (glass and plastic), the photon attenuation (and the strength of its dependence upon photon energy) in these spurious absorbers is greatest for energies below $100 \mathrm{keV}$. 
Owing to the curvature of the vacuum vessel walls, this lower energy radiation will undergo different amounts of attenuation on its way to each of the different detectors, since $x$-rays traveling towards the top-most detector in the stack must pass through a greater thickness of glass and plastic. Secondly, a complication arises in the presence of the photoelectric absorber "K-edge" in lead at $88 \mathrm{keV}$ (see Figure (II-3) in Appendix II). That is, the photon attenuation coefficient is no longer a monotonically decreasing function of photon energy. (This also occurs at the $L, M, N$, etc. " "edges", but these are of far lower energy than the k-edge energy and are of no concern in the energy range of our interest.) This threefold degeneracy in photon energy in the range of $50-150$ $\mathrm{keV}$ must be removed before the energy dependence of the mass attenuation coefficient may be used for X-ray energy determinations.

To avoid both of these effects, the tin absorbcr is laminated together with the lead absorbers to provide strong attenuation of the x-radiation below $100 \mathrm{keV}$. For example, at $100 \mathrm{keV}$, the attenuation in the "lPb" absorber combination is about $5 \times 10^{6}$ times greater than the absorption provided by $1.3 \mathrm{~cm}$ of vessel glass. Also, the $\mathrm{K}$-edge discontinuity in lead is of no consequence owing 
to the strong attenuation of the radiation $\left(I / I_{0}\right.$ $2 \times 10^{-4}$ for $\left.E_{\gamma} \sim 80 \mathrm{keV}\right)$ at the energy where it takes place (the k-edge for tin is at $28 \mathrm{keV}$ ). Hence, the "spurious absorption" effects described above may be truly neglected.

The photomultiplier tubes were direct coupled in output, which required holding the photocathode at the negative high voltage potential and the anode at ground (through $1 \mathrm{k} \Omega$ ). To avoid leakage-current damage to the photomultiplier, the aluminum tube housings were electrically isolated from ground with about $1 \mathrm{~mm}$ of Mylar wrapping. To load the detector signals onto the transmission cables, the photomultiplier tube outputs were coupled to the transmission lines using $50 \Omega$ emitter followers. The electromagnetic interference (notably from the Marx generator/Blumlein oscillations following diode closure) was minimized by transmitting the detector signals to the screen room through solid aluminumjacketed "styroflex" cable. Overall, noise on the cables was held to less than 3-5 mV. Nominally, the photomultiplier tubes were operated at $-540 \mathrm{VDC}$, giving an overall gain of $\Lambda \sim 600$. The detector signals were monitored on Tektronix Type 556 oscilloscopes using Type lAl plugin units (total risetime $\sim 7 \mathrm{~ns}$ ). 
4.4 Theory of Multi-Channel x-ray Absorption Spectroscopy

We begin the discussion of multi-channel absorption spectroscopy with a general expression for the signal intensity from the various $\mathrm{x}$-ray detectors. We may then concern ourselves with the experimental realities as we deal with each term in the signal intensity expression.

\subsubsection{Detector Output Signals: A General Expression}

In general form, the current, $I\left(E_{0}\right)$, appearing at the output of the photomultiplier tube as a function of the energy, $E_{0}$. of the relativistic electrons striking the wire target is given by:

$$
\begin{aligned}
I\left(E_{0}, t\right) & =q_{e} \int_{0}^{E_{O}} d E_{Y}^{\prime \prime} \int_{Y} d_{Y_{n}} \int_{z_{n}} d_{z_{n}} \\
& \times A_{P M T} n_{P C} n_{X T L} \varepsilon_{O}\left(\theta_{D^{\prime}} E_{\gamma}^{\prime \prime}\right) E_{Y}^{\prime \prime} \\
& \times c_{d}\left(x_{N}, Y_{N}, z_{N}, \theta_{D \gamma^{\prime}} x_{C}, Y_{C^{\prime}} z_{C}, x_{D}, Y_{D}, z_{D}\right) \\
& \times S_{Y N}\left(E_{O}, E_{Y}^{n}, \theta_{N \gamma^{\prime}} x_{N}, Y_{N}, z_{N}\right)
\end{aligned}
$$

where

$$
s_{Y i}=T_{i, i-1} s_{Y(i-1)}
$$

and $T_{i, i-1}$ is the transformation of the incident radiation spectrum by the $i^{\text {th }}$ absorber as the radiation 
transits the absorber thickness. Lastly,

$$
\begin{aligned}
& S_{\gamma o}\left(E_{0}, E_{Y}^{\prime}, \theta_{o \gamma}\left(x_{w}, Y_{w}, z_{w}\right), x_{0}, Y_{0}, z_{o}\right) \\
& =\int_{0}^{z_{w}} d z \int_{0}^{y_{w}} d y \int_{0}^{X_{w}} d x \Phi^{\left(x, y, z, t, \theta_{0 e^{\prime}}(x, y, z, t)\right)} \\
& x \frac{d^{2} \sigma_{B R E M S S}}{d \Omega d E_{\gamma}^{\prime}}\left(E_{O^{\prime}}, E_{\gamma}^{\prime}, \theta_{e}^{\prime}(x)-\theta_{o e}^{\prime}, \theta_{\gamma}^{\prime}\right)
\end{aligned}
$$

denotes the $\mathrm{X}$-ray spectrum striking the first absorber. An illustration of the source, absorber, collimator, and detector geometries used in expressions (4.2)(4.4) is shown in Figure (4-4), and the following notations have been used:

$$
\begin{aligned}
& \theta_{\text {oe }}^{\prime} \quad=\text { angle at which the electron strikes } \\
& \text { wire target } \\
& \theta_{e}^{\prime}(x) \quad=\text { local trajectory angle of electron in } \\
& \text { target } \\
& \text { E } \quad=\text { energy of incident electrons } \\
& \theta_{\text {or }}^{\prime} \quad=\text { angle at which Bremsstrahlung photons } \\
& \text { leave targct } \\
& E_{\gamma}^{\prime} \quad=\text { energy of emitted Bremsstrahlung photon } \\
& n_{w} \quad=\text { atomic density in target } \\
& \frac{d^{2} \sigma_{B R E M S S}}{d \Omega d E_{\gamma}^{\prime}}=\text { differential Bremsstrahlung cross- } \\
& \text { section }
\end{aligned}
$$


$-168-$

FIGURE $\quad 4-4$

Illustration of source, absorber, collimator, and detector geometries for use in Equations (4.2) to $(4.4)$. 


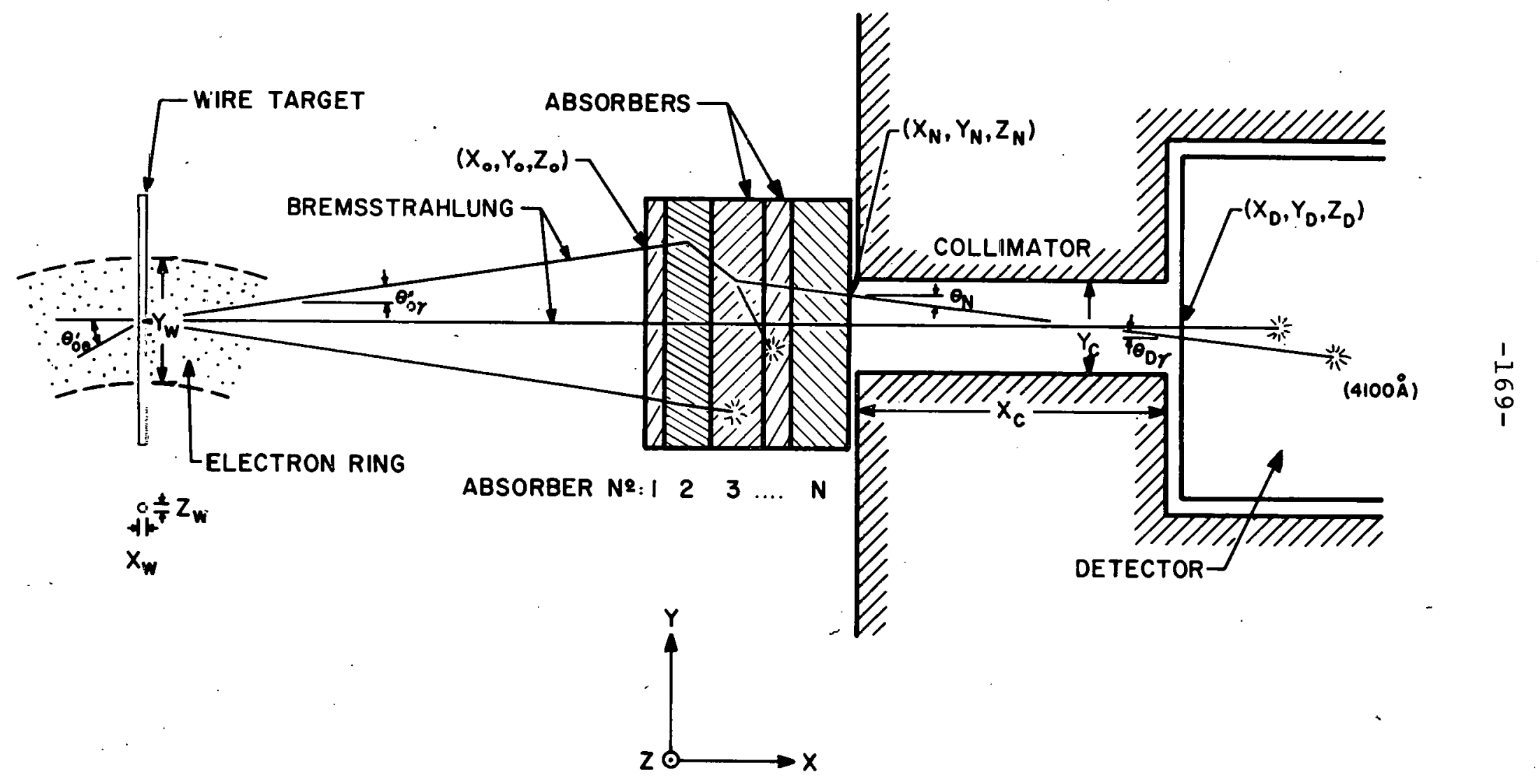

FIGURE 4-4 
$\Phi_{e}\left(x, y, z, t, \theta_{o e}^{\prime}\right)=$ spatial and time dependent electron flux onto target

$X_{w}, Y_{w}, Z_{w}$

$\theta_{o r}^{\prime}\left(x_{w}, y_{w}, z_{w}\right)$

$s_{\text {ro }}$

$s_{\gamma N}$

$x_{N}, y_{N}, z_{N}$

$\mathrm{X}_{\mathrm{C}}, \mathrm{Y}_{\mathrm{C}}, \mathrm{Z}_{\mathrm{C}}$

$\mathrm{x}_{\mathrm{D}}, \mathrm{Y}_{\mathrm{D}^{\prime}}, \mathrm{z}_{\mathrm{D}}$

$C_{D}$

${ }^{\theta}$ Y

$\varepsilon_{0}$

${ }^{n}$ XTL
= target spatial dimensions

$=$ angular distribution of Bremsstrahlung at $\left(x_{0}, y_{0}, z_{0}\right)$

= Bremsstrahlung intensity source function at $\left(x_{0}, y_{0}, z_{0}\right)$

$=$ Bremsstrahlung intensity emitted from rear face of the $N^{\text {th }}$ absorber

= spatial coordinates of rear face of $\mathrm{N}^{\text {th }}$ absorber

= dimensions of collimator

= spatial coordinates of front face of detector

= detector collimator function which discriminates against photons with trajectories outside of collimation acceptance angle

$=$ angle at which photons strike detector = efficiency with which photon energy is deposited in detector

= efficiency with which energy deposited in detector crystal is converted to scintillation light 


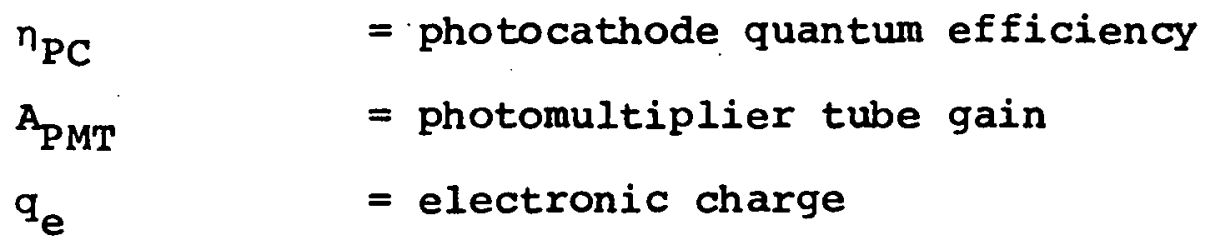

Overall, this set of expressions deals with three main processes:

(i) The production of the Bremsstrahlung spectrum as the fast electrons strike and penetrate the tungsten target;

(ii) the effects of the absorbers on this spectrum, i.e.. intensity photoattenuation, as well as a general "softening" of the energy spectrum owing to compton interactions, and

(iii) the detection of the radiation--which has interacted with the absorbers--by the sodium iodide/ photomultiplier units.

The energy spectrum, angular distribution, and intensity of the Bremsstrahluny, $3_{\text {yo }}$, irradiating a point, $\left(x_{0}, y_{0}, z_{0}\right)$, on the front surface of the first absorber consists of the product of the differential Bremsstrahlung cross-section, the spatial and temporal electron flux distribution striking the target, and the density of atomic scattering sites, integrated over the volume of the wire target.

We then express the effect of the $i^{\text {th }}$ absorber on 
the radiation spectrum and intensity in terms of the transformation operator, $T_{i, i-l^{*}}$ In general, $T$ is a very complicated set of interrelated processes which must include such effects as photoabsorption, Compton scattering, and "delta ray" and Auger electron production, all of which may transpire as photons (with energies less than $1 \mathrm{MeV}$ ) traverse the absorber. For absorbers of the thickness and composition used in this experiment, these manifold processes are extremely complicated so, for the moment, we leave $T$ as a "black box" operator.

If the $x$-ray source function from the rear face of the last (N ${ }^{\text {th }}$ ) absorber in the lamination, $s_{Y N}$, is integrated over the dimensions of the last absorber, $\mathrm{Y}_{\mathrm{N}}$, $\mathrm{Z}_{\mathrm{N}}$, and is convoluted with an expression, $\mathrm{C}_{\mathrm{D}}$, which defines the collimated acceptance angle of the front face of the detector, we obtain the time-dependent $x$-ray photon current intersecting the front face of the sodium iodide detector as a function of position and incidence ("detection") angle. Multiplying this fluence of radiation by the photon energy, $E_{\gamma}^{\prime \prime}$, and the detection efficiency, $\varepsilon_{0}$, gives the rate at which the radiation energy in the interval $\mathrm{E}_{\gamma}^{\prime \prime}+\mathrm{E}_{\gamma}^{\prime \prime}+\mathrm{dE}_{\gamma}^{\prime \prime}$ is deposited in the crystal. 
The current from the photomultiplier tube is then obtained by multiplying this rate of energy deposition in the crystal by the efficiency with which it is converted to the characteristic $4100 \AA$ light ( $\operatorname{NaI}(\mathrm{T} I))$ in the crystal, the photocathode quantum efficiency, the photomultiplier tube gain, and the electron charge, and integrating over all photon energies up to the Bremsstrahlung cutoff energy, $E_{0}$.

In the following sections, we evaluate the various terms in these integral expressions.

\subsubsection{Evaluation of the Bremsstrahlung Source Term}

Several factors play roles in the evaluation of the Bremsstrahlung source spectrum. First, the target is a wire and, for these measurements, we require fairly accurate (3-5\%) Bremsstrahlung spectra from such a wire target in the energy range of $100 \mathrm{keV}$ - $1000 \mathrm{keV}$. Unforlunaleily, most of the existing theoretical work has been done assuming a planar (or "slab") target geometry. Second, as indicated above, the target is energetically neither "thick" nor "thin". Hence, one cannot expect to utilize analytical expressions for thin target Bremsstrahlung ${ }^{70}$ with complete integrity since these relationships do not take multiple scattering or energy loss into account. On the other hand, neither does the average 
electron lose such a fraction of $\mathrm{E}_{\mathrm{O}}$ (50-1008), nor is the target thickness a large enough fraction of the electron range, to permit use of existing thick target formulations $^{71}$ for $x$-ray production. Experimentally, Motz, ${ }^{72}$ Rester and Dance, 73 Aiginger, ${ }^{74}$ Kreuzer and Nakel, 75 and Brysk, et al. 76 have performed Bremsstrahlung cross-section measurements in the energy range of 100-1000 keV. However, only a few isolated electron energies were studied $(180,300$, $380,500$, and $1000 \mathrm{keV})$, and the targets were usually evaporated gold/foils, with thicknesses between $24 \mu \mathrm{g} / \mathrm{cm}^{2}$ and $400 \mu \mathrm{g} / \mathrm{cm}^{2}$ (a tungsten slab target $2.5 \times 10^{-3} \mathrm{~cm}$. thick corresponds to a target thickness of $0.049 \mathrm{~g} / \mathrm{cm}^{2}$ ). The overall experimental accuracies were of the order of 208 .

An alternate approach for obtaining the needed target spectra is via Monte Carlo calculations. vitimately, extensive use was made of the photon and electron transport codes, "MCG" and "MCGE", made available to us through the courtesy of the Los Alamos Scientific Laboratory.

4.4.2.1 Description of the Monte Carlo Transport Codes Complete and detailed descriptions of the transport codes used in this work are available elsewhere. 77-79 
Basically, the interactions which are followed in the transport of the particles are as follows:

I. Electron Transport Code

A. Elastic Nuclear Scattering

B. Inelastic Electron-Electron Scattering

1. Density Effects

2. Delta Rays

3. Characteristic $\mathrm{X}$-radiation

C. Bremsstrahlung

D. Annhilation Radiation

II. Photon Transport Code

A. Compton Scattering

B. Photoelectric Absorption

C. Fluorescence and Auger Electrons

D. Pair Production

A brief discussion of the individual formulations used to handle the processes listed above, along with a reference bibliography, appear in Appendix III.1.

Initially, the electron transport code was used to calculate the Bremsstrahlung spectra from the wire target. Subsequently, the photon transport code was adapted for use in computing the sodium iodide detection efficiencies, the absorber transmission coefficients, and to determine the significance of the Compton effect in the absorbers on the wire Bremsstrahlung spectrum. These last three applications shall be discussed in subsequent sections.

A full description of the target geometry used in the codes appears in Appendix III.3. Briefly the 
cylindrical geometry of the wire target was approximated by a lamination of ten semi-infinite tungsten slabs ranging in thickness from $0.0025 \mathrm{~mm}$ to $0.025 \mathrm{~mm}$. The full differential Bremsstrahlung spectrum was computed for each thickness of the laminations forming the target. The final Bremsstrahlung spectrum for the wire was a composite of the individual slab spectra, each weighted according to its lateral geometric contribution to the wire's actual, cylindrical cross-section. The individual spectra were computed in this way for the wire from $100 \mathrm{keV}-1000 \mathrm{keV}$ in $50 \mathrm{keV}$ energy increments. Typically, to achieve good statistical accuracy, the number of electron histories followed ranged from 6700 in the lower energy ranges to over 37,000 at $1 \mathrm{MeV}$.

As discussed in the Appendix, computer runs were also performed utilizing the full cylindircal wire geometry to check on the veracity of the "weighted slab" approximation. The slab approximation was found to be in good agreement with the results from the true wire geometry, within the accuracy of code statistics.

For reference, the tabulated data for the slab Bremsstrahlung spectra appear in Appendix III.5, with accompanying explanations for their use in other applications. 
4.4.2.2 Angular Dependences of the Bremsstrahlung Spectra

of first consideration in the Bremsstrahlung spectrum evaluations was the angular dependence of the spectra as a function of photon energy. The purpose was twofold:

(1) To determine whether the angular separation between the three detectors would result in a significantly different spectrum being observed by each, given the same primary electron energy, and

(2) to discern whether, as suspected for a target of this thickness, the computations in the source integral, Equation (4.4), could be simplified by taking advantage of relationships between the primary electron energy and the transmitted photon angular distribution.

As shown from the data in Appendix III.3, the bulk of the contributions to the composite $x$-ray spectrum from the wire comes from the lamination thicknesses ranging from the $0.025 \mathrm{~mm}$ slab (298 of the total) to the $0.0 .15 \mathrm{~mm} \mathrm{slab}$ ( 88 of the total). Therefore, let us consider the angular distribution of Bremsstrahlung from these two thicknesses of target since, between these limits of slab thickness, about 858 of the total target Bremsstrahlung is generated.

The angular distributions for the Bremsstrahlung 
spectra from these two slab thicknesses are plotted in Figures (4-5) through (4-13) for primary electron energies of $150,300,500,700$, and $1000 \mathrm{keV}$, and are parameterized to photon energies of $100,200,300, \ldots$, kev. For the sake of direct comparison for each primary electron energy, the spectra have been normalized to total radiation intensity in each incremental energy bin. To improve statistics, the spectra were averaged over the indicated photon energy $\pm 50 \mathrm{keV}$. The first salient feature of these plots is the angular width at half the value of the peak of the spectral distribution, $\theta_{\gamma \text { FWHM }}$. For $500 \mathrm{keV}$ electrons striking a $2.54 \times 10^{-3} \mathrm{~cm}$ thick target, the code predicts $\theta_{\gamma \text { FWHM }} \sim 68^{\circ}$. This value was roughly checked using Bethe's large-angle extension of Moliere's multiple-scattering theory, ${ }^{80}$ which brings that theory into fair agreement with the (exact) Goudsmit-Saunderson formulation. 81 For this calculation, $500 \mathrm{keV}$ eleotrons scattering through a tungsten foil $2.54 \times 10^{-3} \mathrm{~cm}$ thick were found to have an angular distribution whose FWHM was $\theta_{\text {eFWHM }}-81^{\circ}$, with $\theta_{\text {eFWHM }}$ directly dependent upon the square root of the target thickness. From the Monte Carlo calculations, we find that about $80 \%$ of the Bremsstrahlung radiated into the forward $2 \pi$-hemisphere is produced by interactions within the first 708 of the 


\section{FIGURE $4-5$}

Comparison of the angular distribution of Bremsstrahlung from a $1.52 \times 10^{-3} \mathrm{~cm}$ tungsten slab with that 'of a $2.54 \times 10^{-3} \mathrm{~cm}$ tungsten slab; $\mathrm{E}_{\mathrm{O}}=150 \mathrm{keV}$. 


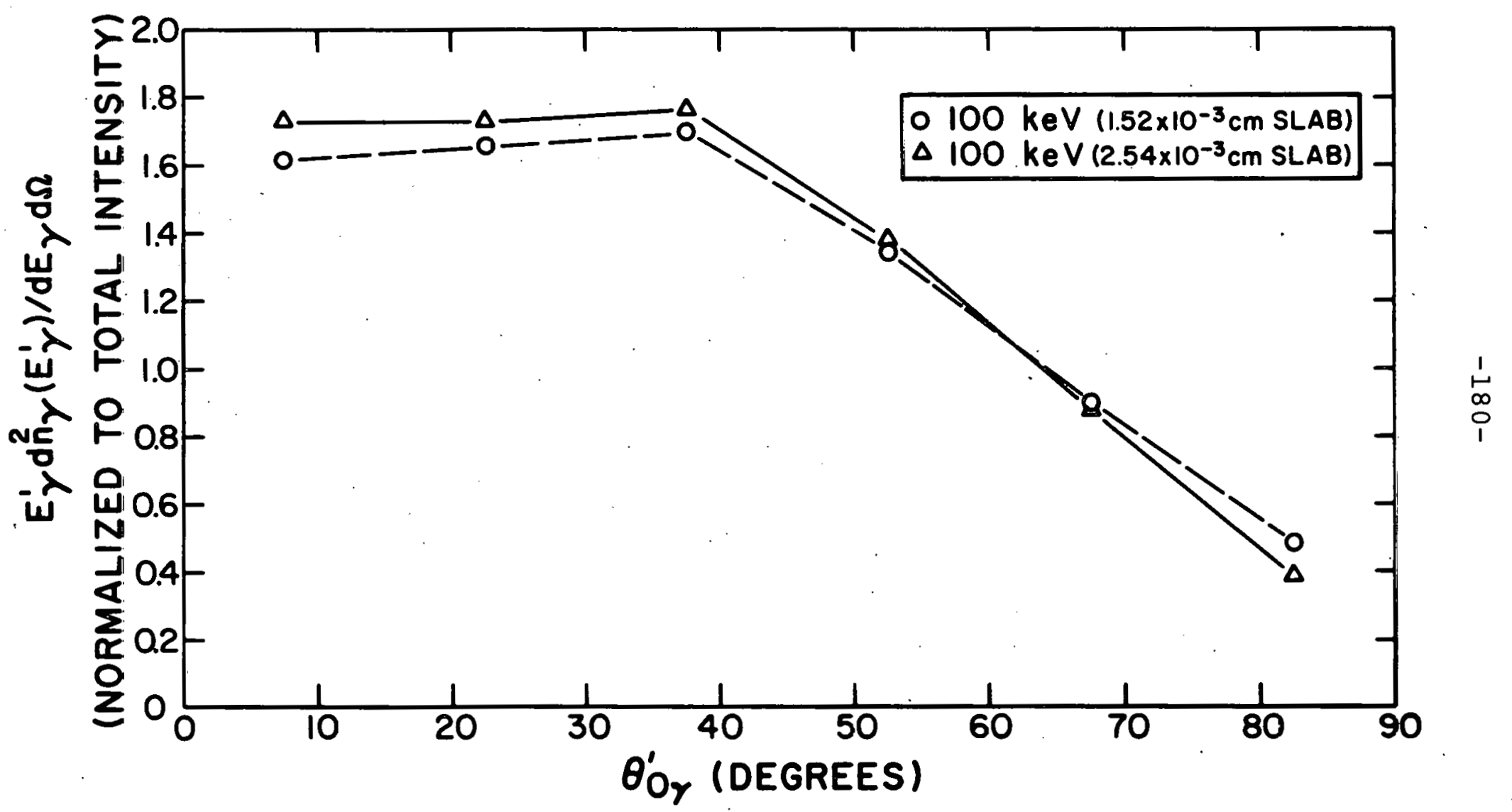

FIGURE 4-5 
FIGURE 4-6

Angular distribution of Bremsstrahlung from a $1.52 \times 10^{-3} \mathrm{~cm}$ tungsten slab; $\mathrm{E}_{\mathrm{O}}=300 \mathrm{keV}$. 


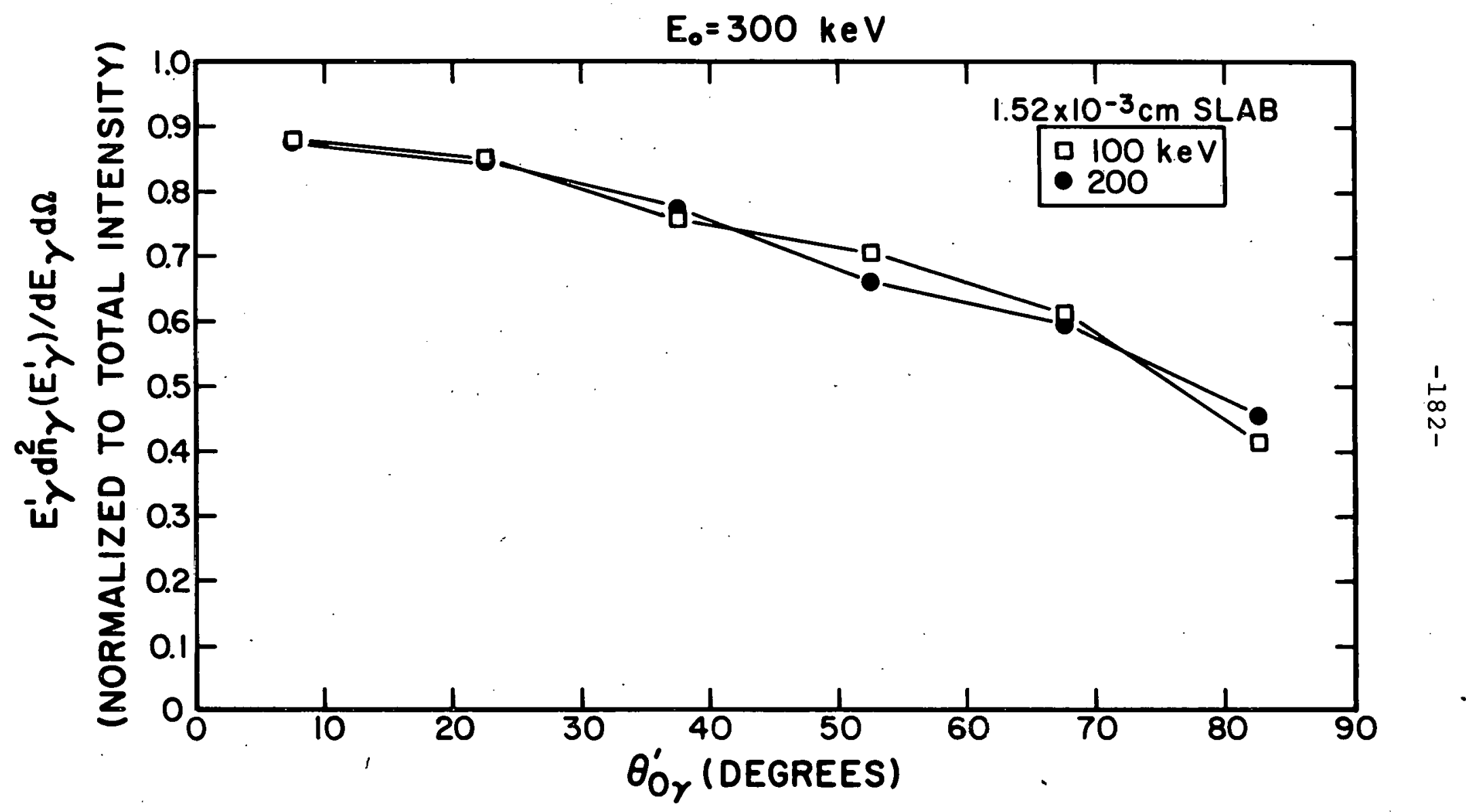

FIGURE 4-6 
FIGURE $4-7$

Angular distribution of Bremsstrahlung from a $1.52 \times 10^{-3} \mathrm{~cm}$ tungsten slab; $E_{0}=500 \mathrm{keV}$. 


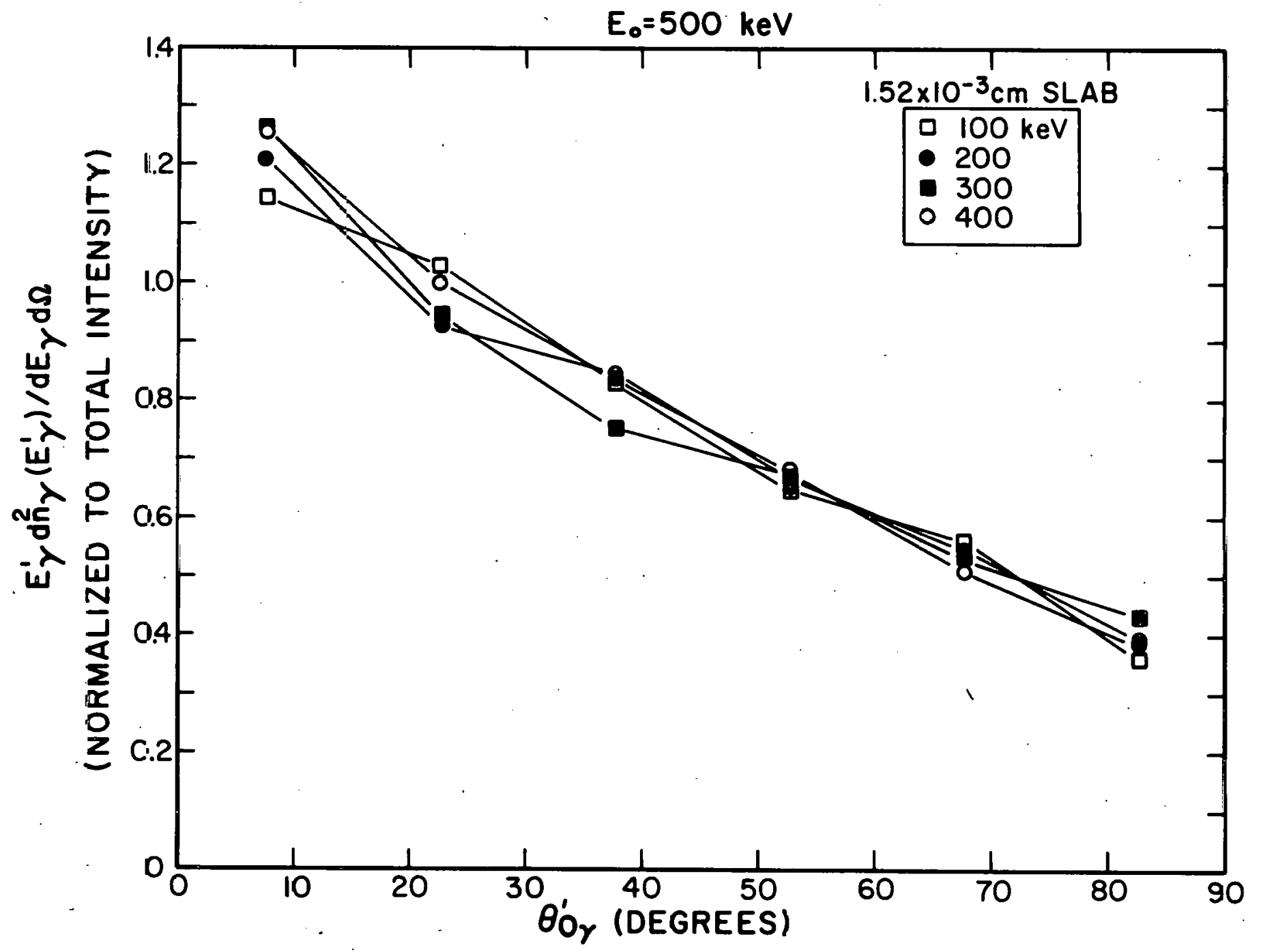


FIGURE $4-8$

Angular distribution of Bremsstrahlung from a $1.52 \times 10^{-3} \mathrm{~cm}$ tungsten slab; $E_{0}=700 \mathrm{keV}$. 


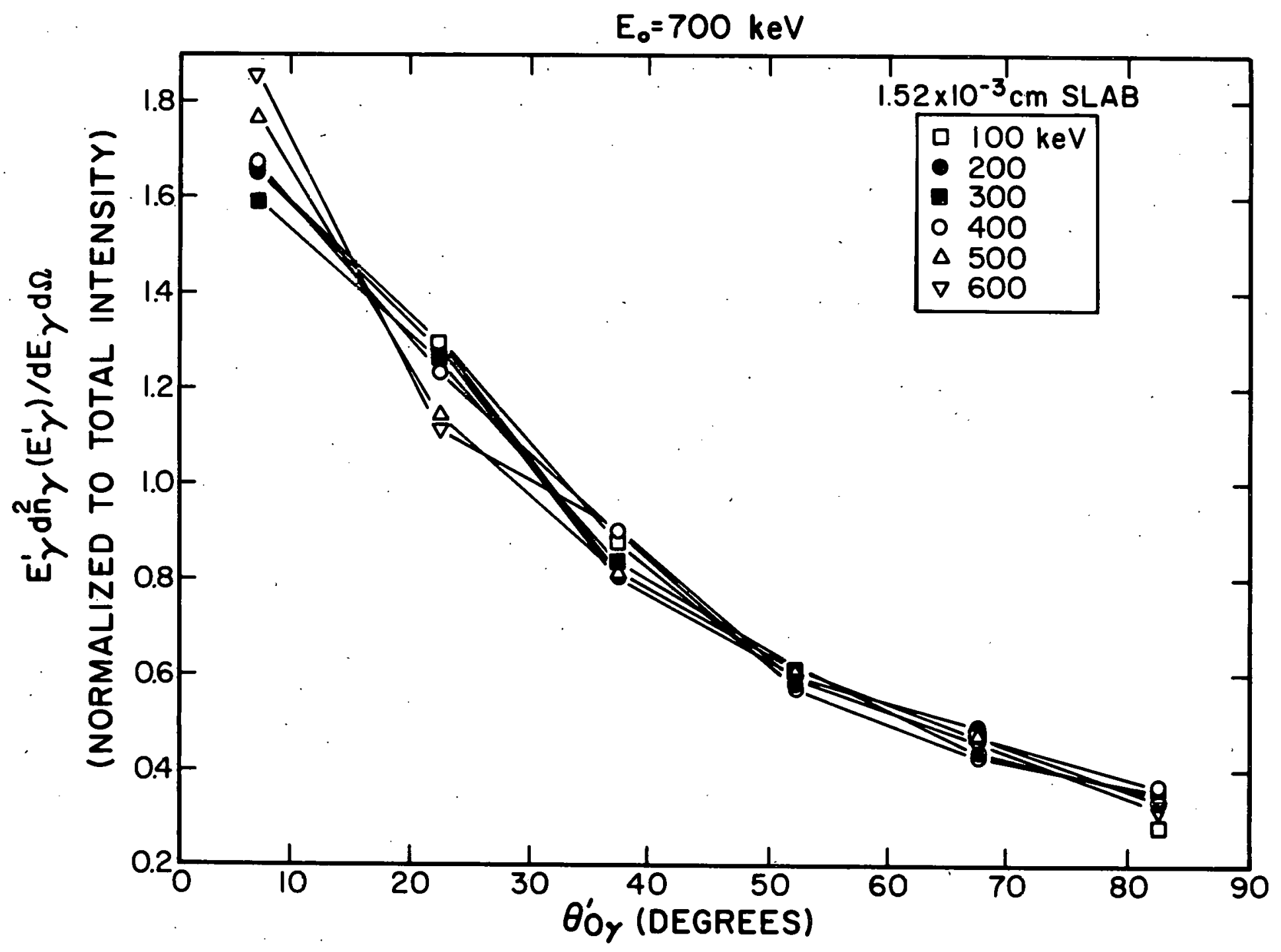

1
1
$\infty$
0
1

FIGURE 4-8 
Angular distribution of Bremsstrahlung from a

$1.52 \times 10^{-3} \mathrm{~cm}$ tungsten slab; $\mathrm{E}_{\mathrm{O}}=1000 \mathrm{keV}$. 


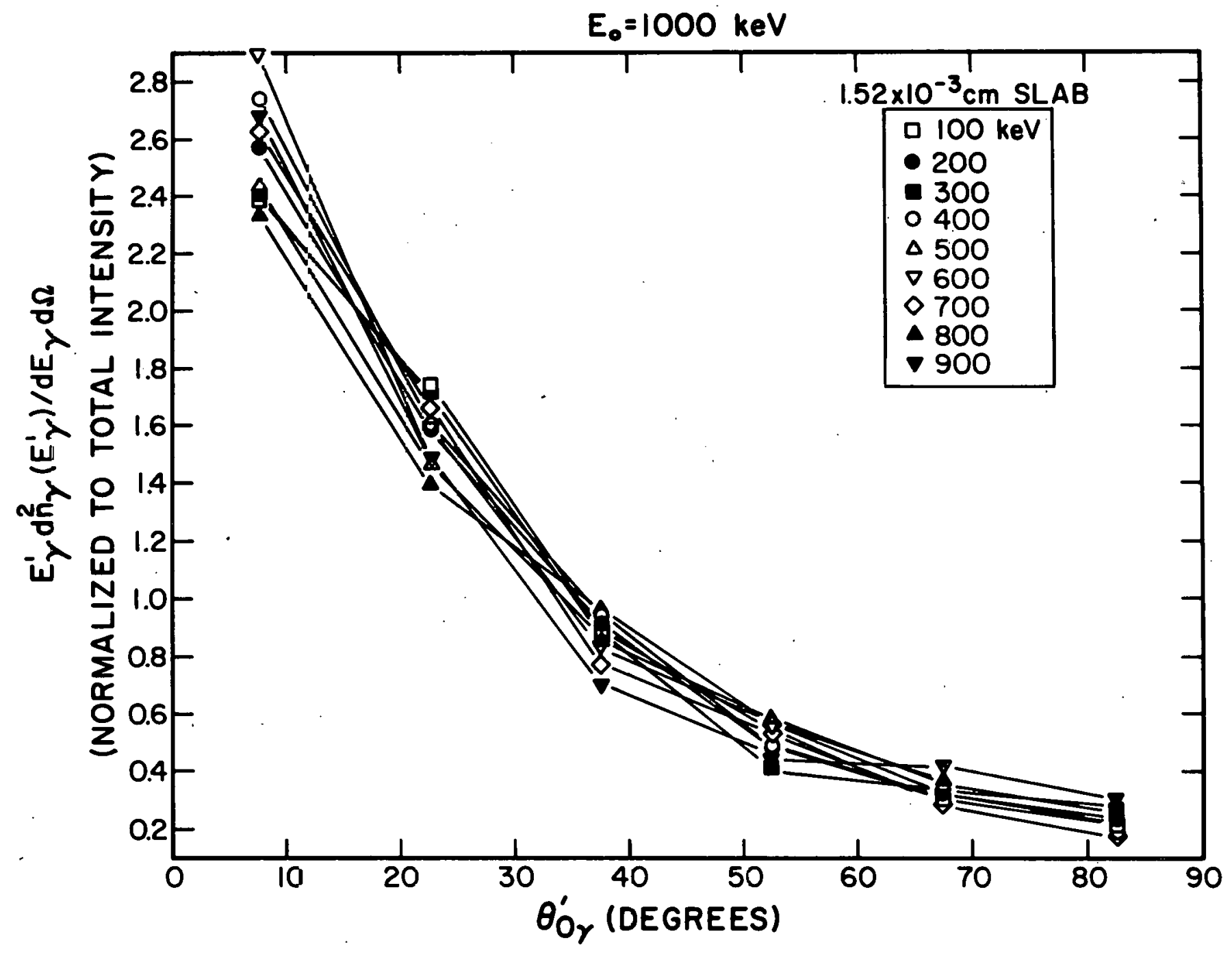

1
1
$\infty$
0
1

FIGURE 4-9 


\section{FIGURE 4-10}

Angular distribution of Bremsstrahlung from a $2.54 \times 10^{-3} \mathrm{~cm}$ tungsten slab; $\dot{\mathrm{E}}_{\mathrm{O}}=300 \mathrm{keV}$. 


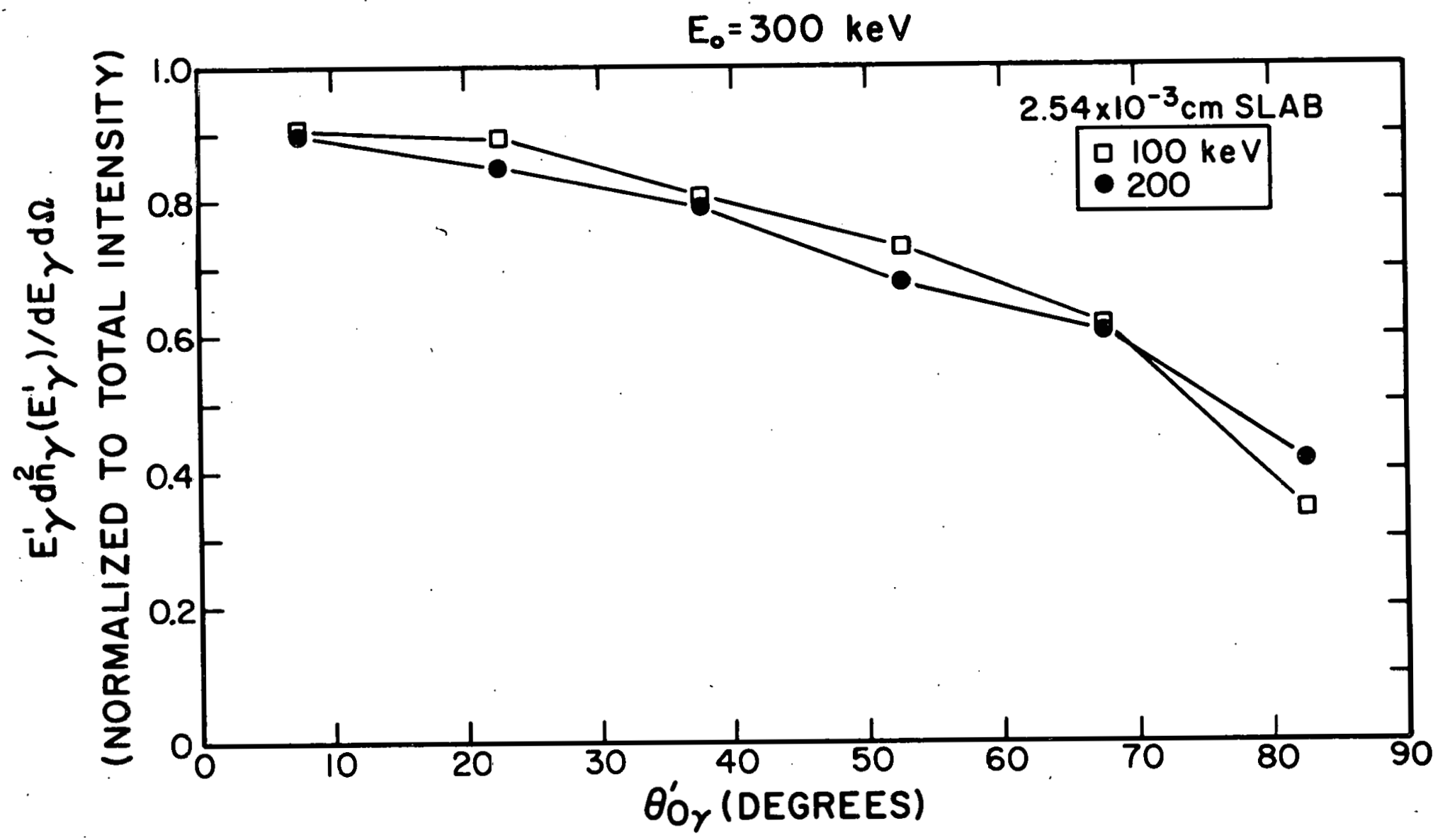


FIGURE $4-11$

Angular distribution of Bremsstrahlung from a $2.54 \times 10^{-3} \mathrm{~cm}$ tungsten slab; $\mathrm{E}_{\mathrm{O}}=500 \mathrm{keV}$. 


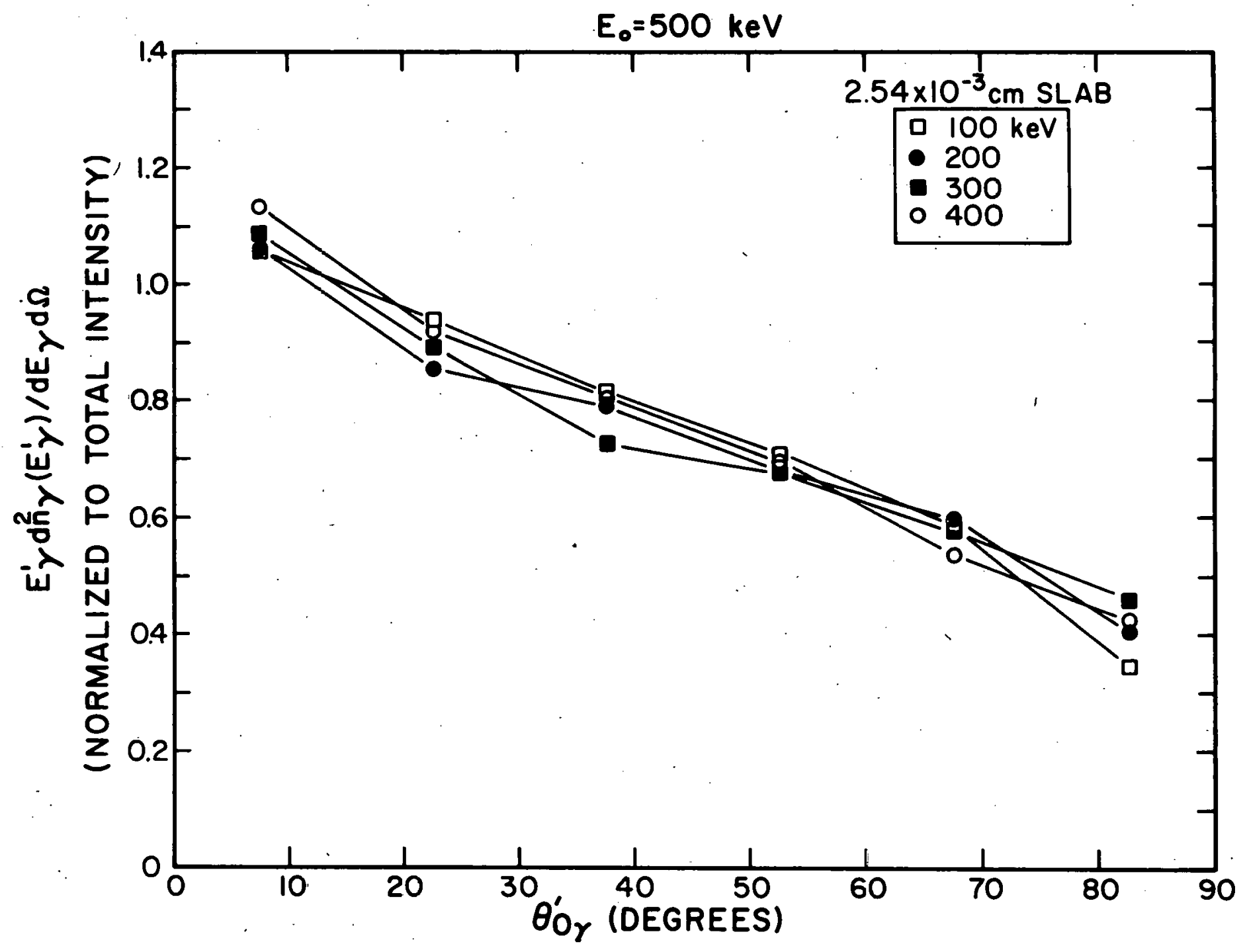

FIGURE 4-11 
FIGURE 4-12

Angular distribution of Bremsstrahlung from a $2.54 \times 10^{-3} \mathrm{~cm}$ tungsten slab; $E_{0}=700 \mathrm{keV}$. 


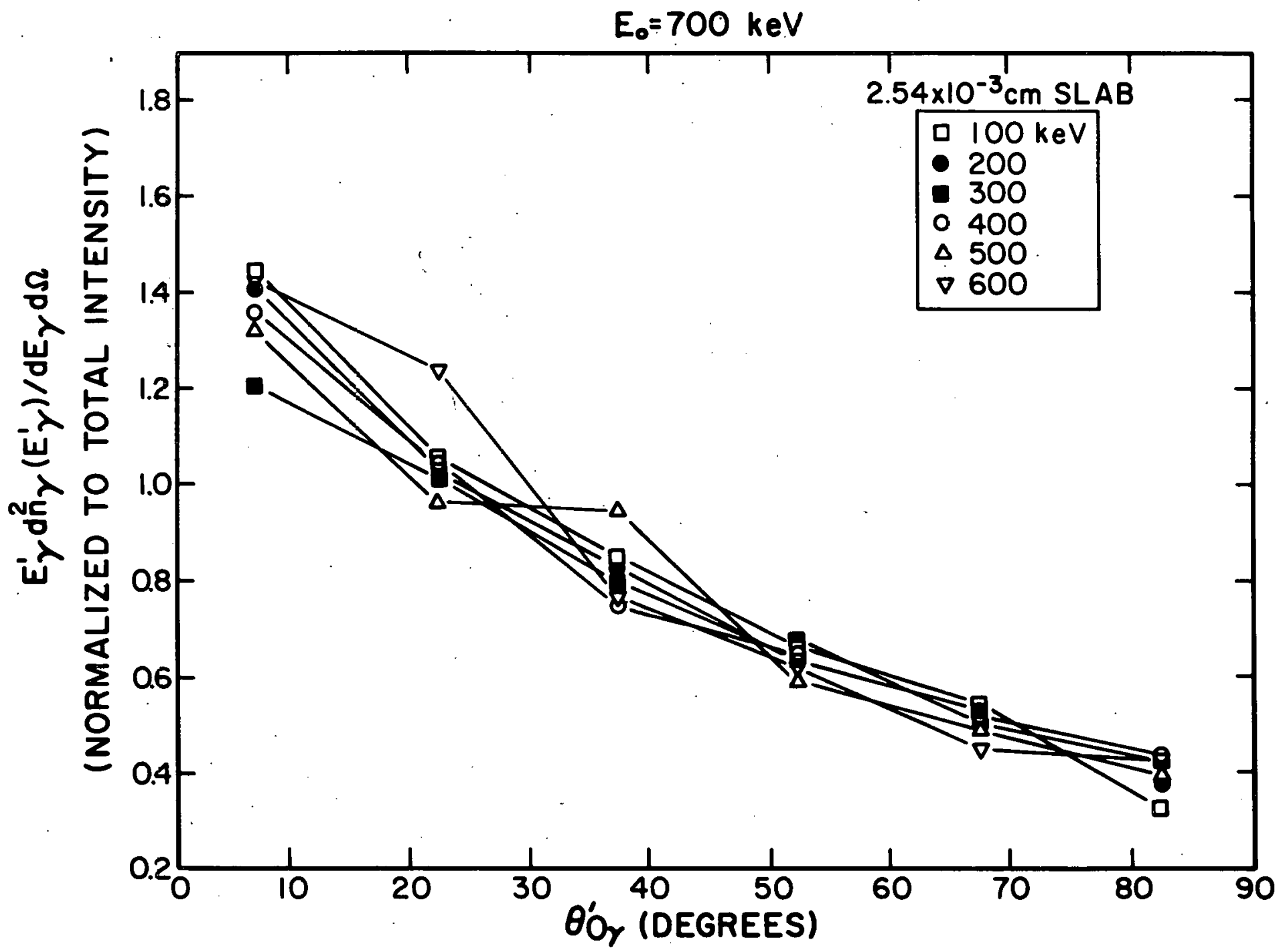


FIGURE $4-13$

Angular distribution of Bremsstrahlung from a $2.54 \times 10^{-3} \mathrm{~cm}$ tungsten slab; $\mathrm{E}_{0}=1000 \mathrm{kev}$. 


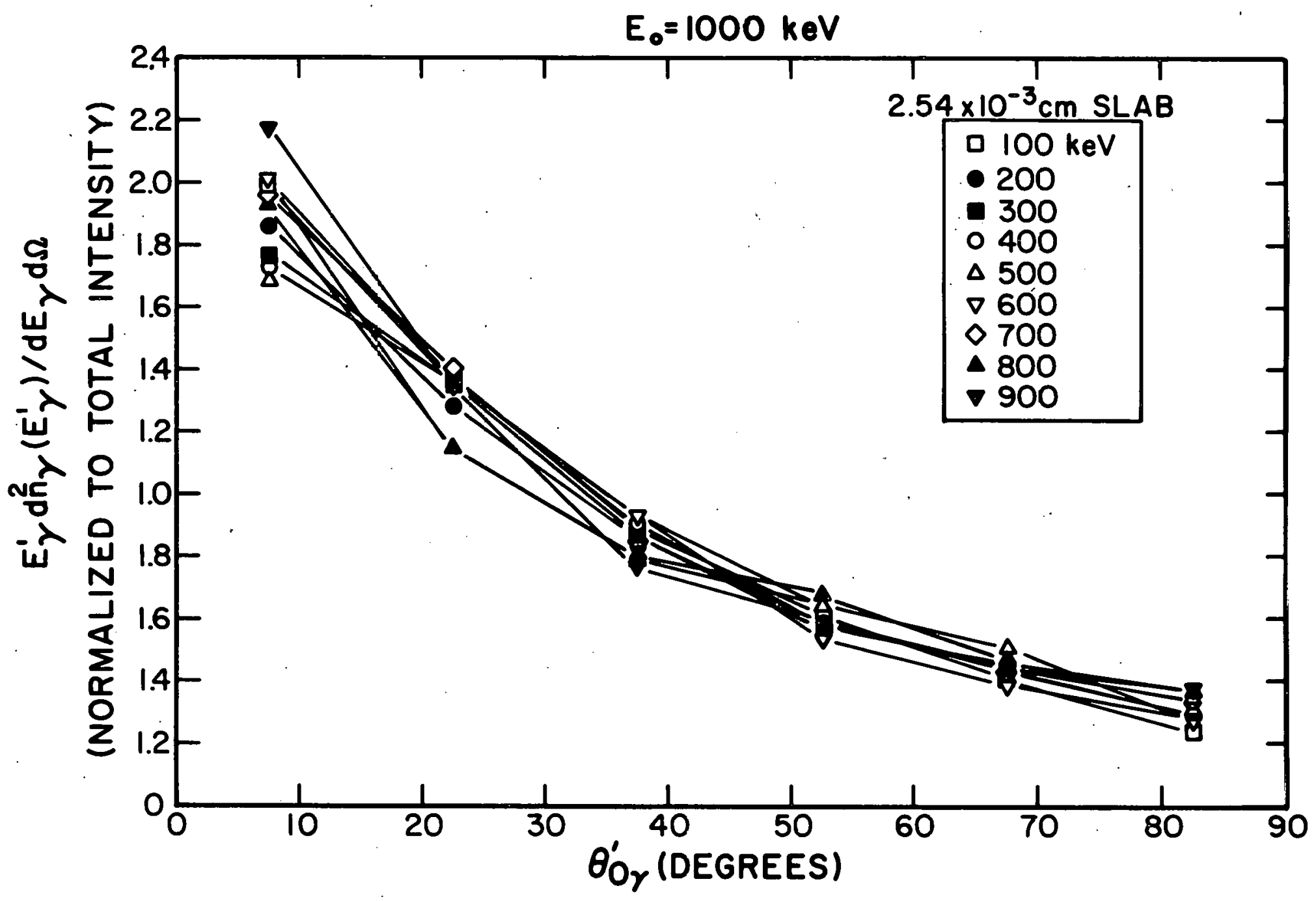


target thickness. Therefore, the rate of $\mathrm{x}$-ray production is roughly constant over the thickness of the slab, and one may compute the average electron scattering angle responsible for the Bremsstrahlung production as $\left\langle\theta_{e}>-81^{\circ} / \sqrt{2}-57^{\circ}\right.$, where we have used $<\theta_{e}^{2}>=\int \theta_{e}^{2}(x) d x / \int d x$. From the thin-target Bremsstrahlung measurements of Motz, 82 in which $500 \mathrm{keV}$ electrons scattered in gold targets $4.5 \times 10^{-4} \mathrm{~cm}$ thick $(\sim 0.18$ range fraction)', the half-width for the Bremsstrahlung cross-section angular distribution for single scatterings was found to be $\theta_{\gamma F W H M} \sim 25^{\circ}$. Thus, we estimate that the net FWHM of the Bremsstrahlung spectrum from our target should be about $\left(57^{2}+25^{2}\right)^{1 / 2}-62^{\circ}$, which is in reasonable agreement with the code prediction of $68^{\circ}$.

Therefore, the angular separation of the detectors, $7^{\circ}$, is much less than $\theta_{\gamma \text { FWHM }}\left(\sim 70^{\circ}\right)$. The Bremsstrahlung radiation pattern is "smeared out" to the extent that the three detectors can confidently be expected to "see" the same Bremsstrahlung spectra. In addition, the angular divergence of the $\mathrm{x}$-rays--and the uniformity of the spectra--are further enhanced by the angular divergence of the ring electrons (which, for strong electron rings, is probably in the neighborhood 


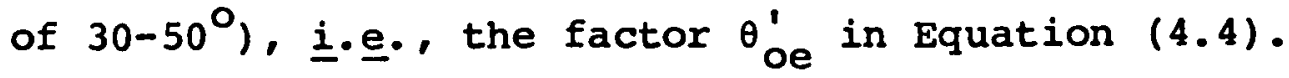

Furthermore, the plots of Figures (4-5) - (4-13) indicate that, for a given primary electron energy, the angular dependence of the Bremsstrahlung spectra is essentially photon-energy independent. Hence, the angular dependence of the source term, $\mathrm{s}_{\text {yo' }}$ in expression (4.4) may be removed from the integral as a constant factor. Since we shall eventually be taking ratios of photomultiplier current signals a $\underline{\text { la }}$ expression (4.1), the nature of the constant factor is unimportant in our computations. Therefore, it is acceptable to use the total Bremsstrahlung spectra integrated over the forwardscattered $2 \pi$-hemisphere. Statistically, this is a much more accurate utilization of the existing codes than to use the computed values for the spectral distribution differential in angle and energy, in which the relative error for individual terms at small angles and large energies (near cut-off) can be substantial (20-50\%). The code-generated spectra integrated over the forward $2 \pi$-hemisphere for the wire target are shown in Figure $(4-14)$.

\subsubsection{Absorber Transmission Factors}

The questions of photo- and Compton effects in the absorbers are closely tied together with the detector 


\section{FIGURE 4-14}

Bremsstrahlung spectra from a $2.54 \times 10^{-3} \mathrm{~cm}$ diameter tungsten wire, integrated over the forward $-2 \pi$ hemisphere. 


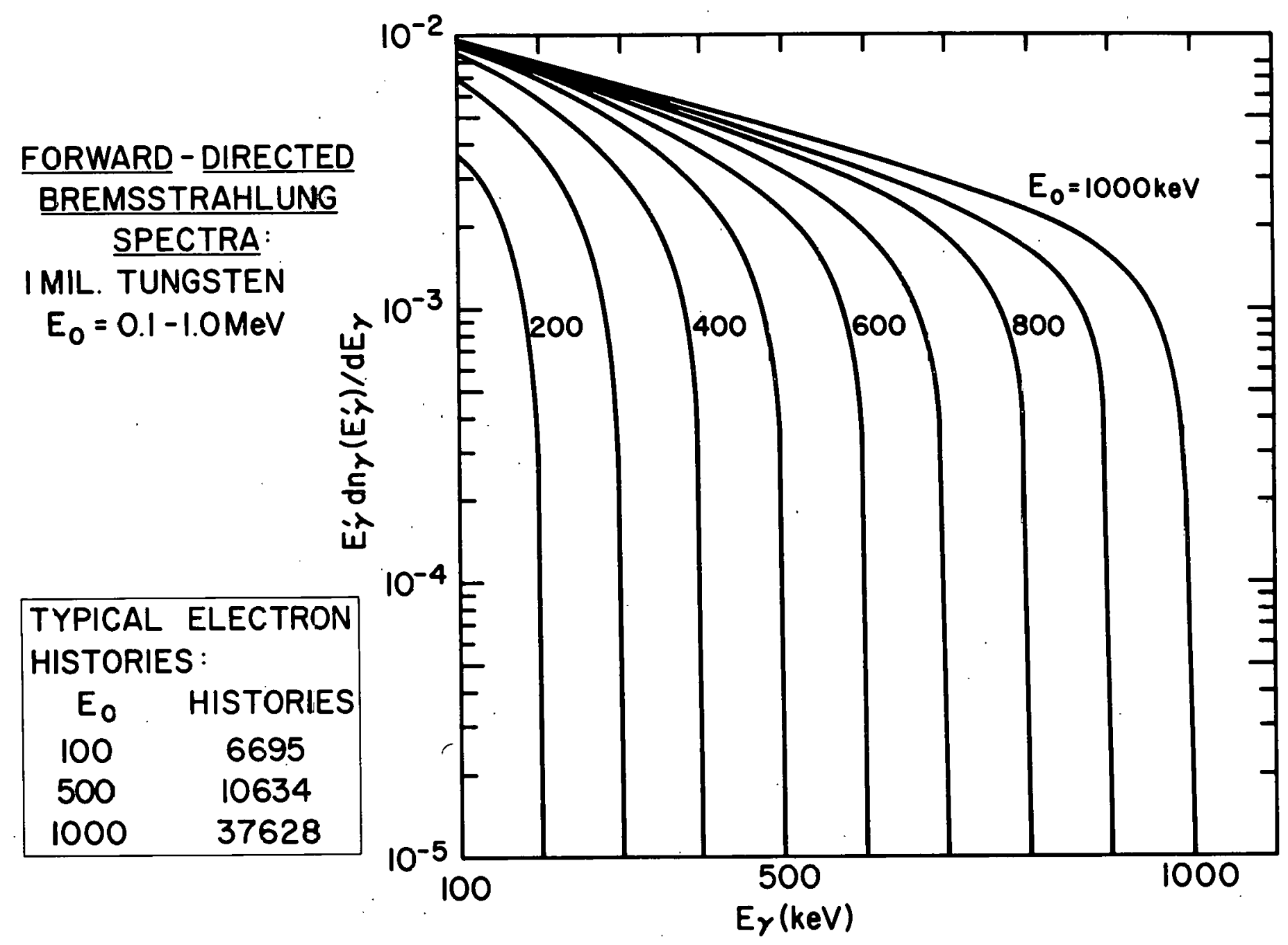


collimation geometry. If the detector is positioned close enough to the absorbers to subtend a large solid angle relative to the rear face of the absorbers, one may expect a significant "softening" of the detected $\mathrm{x}$-ray spectrum owing to the detection of a sizeable quantity of Compton-scattered photons in addition to the photoattenuated fluxes.

The collimation provided between the absorbers and the front face of the detectors was designed in anticipation of this problem. Referring to Figure (4-4), the dimensions of the collimator were $\mathrm{x}_{\mathrm{C}}=15 \mathrm{~cm}, \mathrm{Y}_{\mathrm{C}}=\mathrm{z}_{\mathrm{C}}=$ $1.27 \mathrm{~cm}$. Therefore, from the rear face of the absorber package, the front face of the detector at the other end of the collimator tunnel subtends a solid angle of $-5.5 \times 10^{-3} \mathrm{Sr}$. This small solid angle results in quite effective discrimination against compton-scattered photons, since the probability is extremely low that singly-scattered Comptons suffering a significant decrease in energy could subtend the acceptance solid angle of the detector. As discussed in Appendix (III.6), the effect of Compton scattering--as calculated from the Klein-Nishina formulation ${ }^{83}$--on the spectral distribution of the target Bremsstrahlung reaching the detector for our collimation geometry is seen to amount to less than 
a $0.1 \%$ effect in the energy range of interest. This prediction is in agreement with the transport code results in which the full collimation geometry has been taken into account. In view of the small size of this effect, one may also neglect radiation coming from other parts of the RECE-Berta device that would not normally reach the detectors on a collimated line-of-sight basis, but which still may enter the detectors' collimated acceptance angle due to scattering. This is confirmed experimentally, after the first microsecond following beam injection (ㄷ․ Section 4.5.2).

\subsubsection{Absorber Transmission Coefficients}

In light of the neglect of Compton effects on the spectrum of detected radiation, as described in the preceeding section, for our purposes and accuracy virtually all photons which interact with the target are removed from the $x$-ray flux observed by the detectors. Thus the transmission coefficients are the same as those calculated from the photon mass attenuation coefficients. This result brings about a significant simplification of Equations (4.3) and (4.4) above. That is, the absorber transformation operator, $T_{i, i-1}$, can effectively be replaced by delta functions in energy and angle for all absorbers: 


$$
T_{i, i-1}=\delta\left(E_{\gamma}^{n}-E_{\gamma}^{\prime}\right) \delta\left(\theta_{o \gamma}^{\prime}-\theta_{o \gamma}\right) e^{-\mu_{i}^{a}\left(E_{\gamma}^{\prime}\right) / \cos \left(\theta_{o \gamma}^{\prime}\right)}
$$

and $S_{\gamma N}$ in Equation (4.2) may be rewritten as

$$
\begin{aligned}
& s_{\gamma N}\left(E_{0}, E_{\gamma}^{\prime \prime}, \theta_{N \gamma}, X_{N}, Y_{N}, z_{N}\right) \\
& =s_{\gamma_{0}}\left(E_{0}, E_{\gamma}^{\prime}, \theta_{0}^{\prime}, x_{0}, y_{0}, z_{0}\right) \\
& x \prod_{i=1}^{n} e^{-\mu_{i}^{a}\left(E_{\gamma}^{\prime}\right)\left(\Delta x_{i} / \cos \left(\theta_{o \gamma}^{\prime}\right)\right)}
\end{aligned}
$$

where $\Delta x_{i}$ is the thickness of the $i^{\text {th }}$ absorber. That is, all absorber interactions with the photon flux are assumed to remove the interacting photons from the detected flux. In this context, $\mu_{i}^{a}\left(E_{\gamma}^{\prime}\right)$ is now the total mass-attenuation coefficient for the $i^{\text {th }}$ absorber.

The photon transport codes were utilized to obtain the attenuation of the Bremsstrahlung through the conducting copper liner and Lexan damage liners in Berta, the glass wall of the vacuum vessel, the various thicknesses of lead absorber used in the measurement, the tin absorber, and the copper wall of the Faraday cage housing the photomultiplier array. The results of the transmission coefficient calculations are plotted in Figure (4-15) as 
FIGURE $4-15$

Absorber transmission coefficients for " 0 ", "1", "2", and "3" $\mathrm{Pb} / \mathrm{Sn}$ absorbers. 


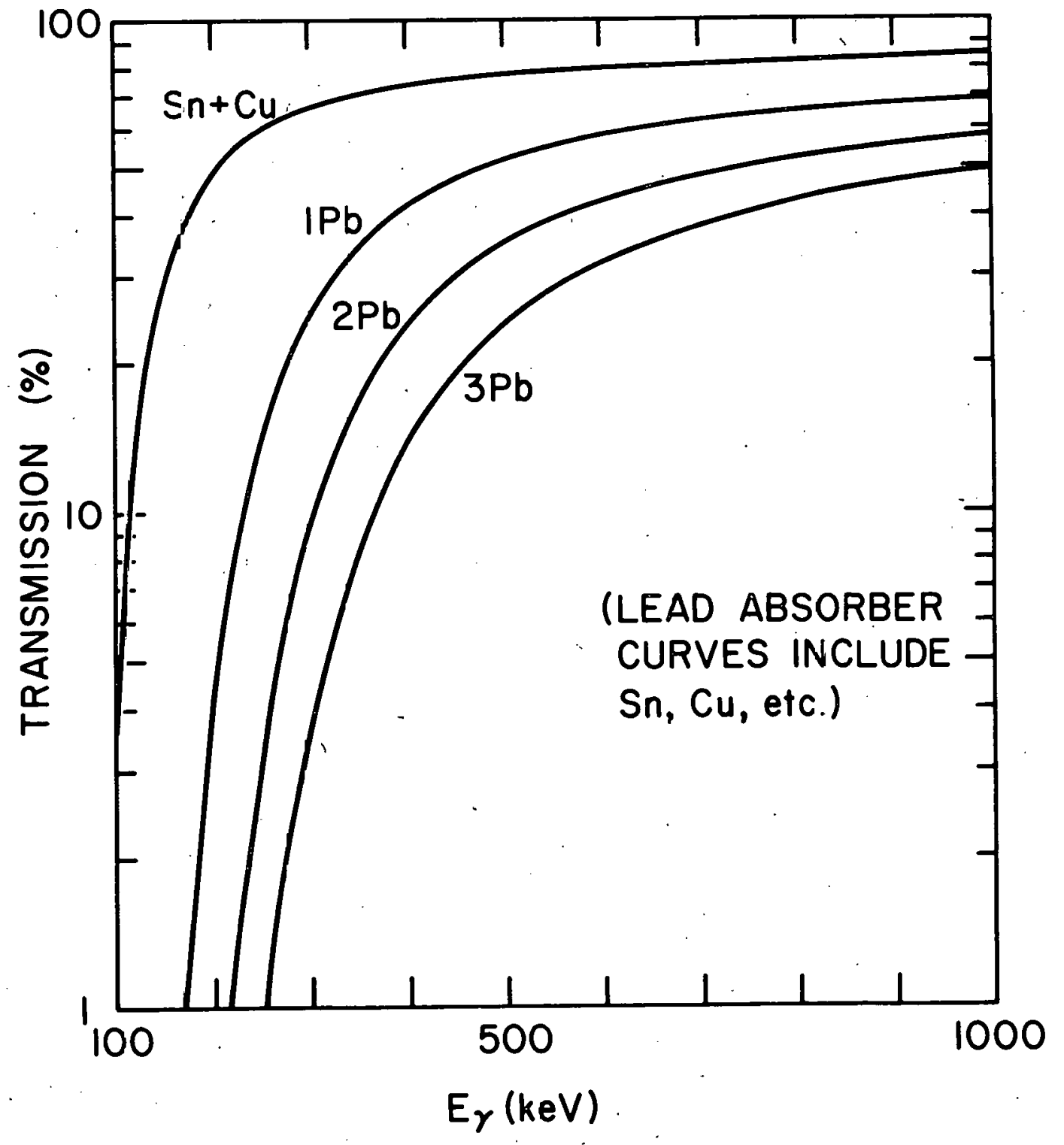

FIGURE 4-15 
"I Transmission" of photons through the absorbers as a function of photon energy. As indicated in the plots, the addition of the tin attenuates the fractional photon intensity transmission to a level well below 0.18 for energies less than $100 \mathrm{keV}$, eliminating the effects of the lead K-edge absorption degeneracy.

As expected from the energy dependence of the massabsorption coefficient, the slopes of the transmission curves flatten out considerably at energies greater than $500 \mathrm{keV}$ : at $500 \mathrm{keV}$, the photoabsorption in lead accounts for about 50 of the total attenuation, while at $1 \mathrm{MeV}$, it accounts for only about 30z--with compton scattering accounting for the remainder of the attenuation.

The results of the computer code computations were consistently within $2-4 \%$ of the results of the exponential attenuation as calculated from the mass-attenuation coefficients, the accuracy being that with which the photon attenuation coefficients could be read from the plots such as those of Appendix II.

\subsubsection{Sodium Iodide Detector Efficiencies}

As mentioned above, the $\mathrm{X}$-ray detectors used in this work were right-cylindrical, thallium-doped sodium iodide crystals, $4.5 \mathrm{~cm}$ in diameter and $5.1 \mathrm{~cm}$ long. The overall response of such detectors to gamma irradiation 
consists of four independent factors:

(1) The efficiency with which the energy of the radiation incident on the front face of the detector is deposited in the crystal;

(2) the efficiency with which that energy deposited within the body of the crystal is converted into scintillation light;

(3) the quantum efficiency with which that light striking the photocathode of the photomultiplier tube produces photoelectrons from the photocathode surface, and

(4) the overall gain of the photomultiplier tube. The last three factors listed above are measured constants usually available from the manufacturer's data and the physical characteristics of the crystal. The efficiency with which radiation energy deposited in the crystal is converted to transmission-band light is $12 \%$ in sodium iodide. 84 The scintillation light, emitted at a wavelength of $4100 \AA$, falls near the peak of the spectral response curve of the photomultiplier tube used. The photocathode efficiency for incident radiation of $4100 \AA$ is $15.5 \%$ (S-11 phosphor).$^{85}$ Finally, the gain of the tube, operated at $-540 \mathrm{VDC}$, was $\sim 600 .^{85}$ (The emitter-followers, mounted on the bases of the 
photomultiplier tubes, had matched gains of 0.60 each). The detector energy-deposition efficiency, however, depends in detail on the specific configuration in which it is to be used. Calculations, 86 as well as some experimental work ${ }^{87}$ have been performed to obtain NaI(TI) crystal response efficiencies for various geometries of sources and for various detector sizes, all parameterized to photon energy. However, most of the work has assumed uniform detector irradiation without collimation (which geometry is of practical use in numerous isotope spectroscopy applications). In order to account for both the collimation effects as well as the finite detector geometry, extensive use was made once again of the photon transport codes to calculate the detection efficiency via Monte Carlo techniques.

The details of the crystal geometry used in the code calculations are presented in Appendix III.2. The plot of detector energy-deposition efficiency as a function of photon energy as obtained from the code calculations appears in Figure (4-16). At energies in the range of 100-200 kev, the efficiency was found to be about 98\%, but drops sharply (largely due to the loss of the photoelectric cross-section in iodine) to efficiencies of around $44 \%$ at $1 \mathrm{MeV}$. 


\section{FIGURE $4-16$}

X-ray detection efficiency for a right-cylindrical

Sodium Iodide crystal $4.5 \mathrm{~cm}$ in diameter, $5.1 \mathrm{~cm}$ long. 


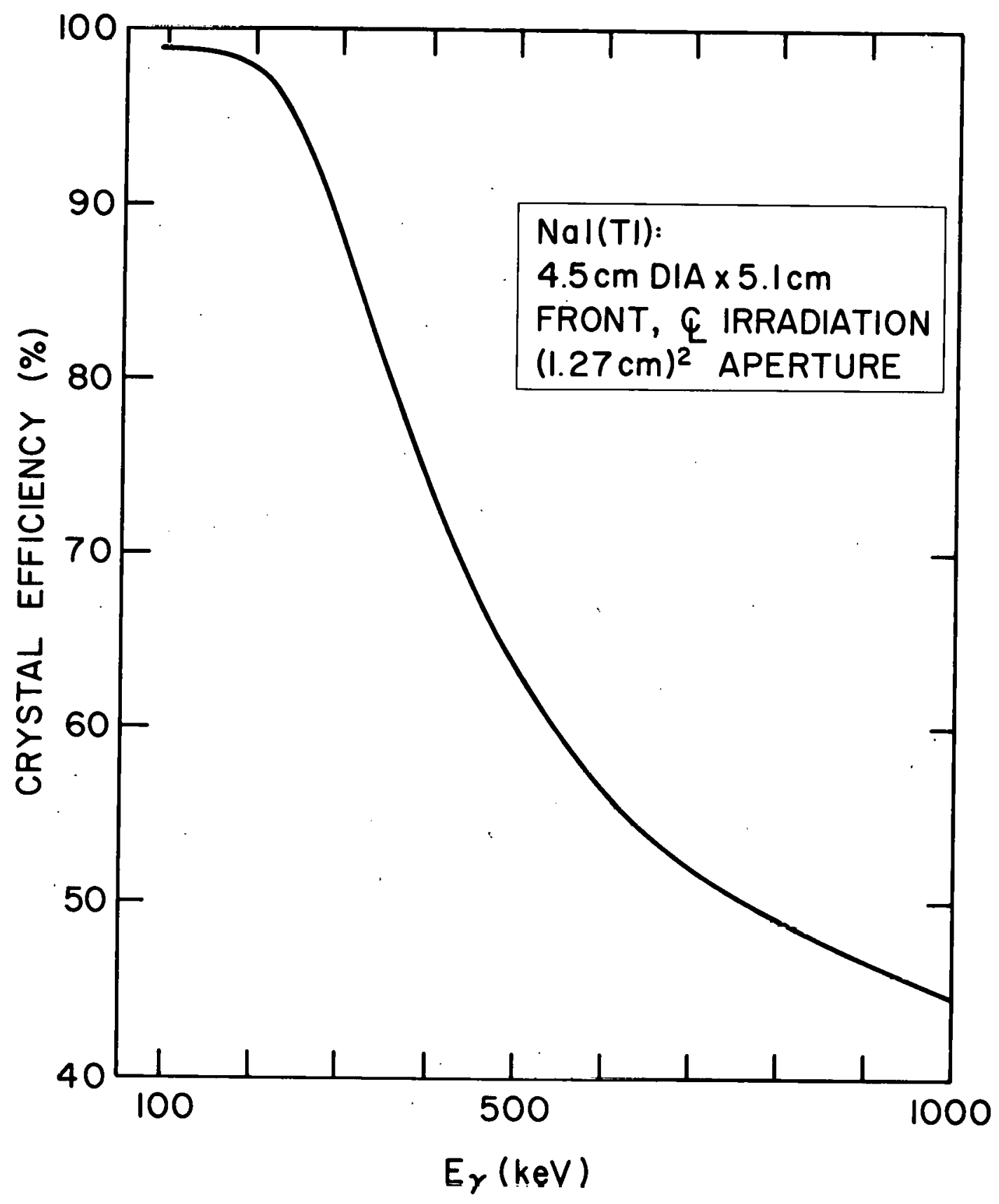

FIGURE 4-16 
These results agree quite closely (within 6-10\%) with first-order efficiency estimates described in Appendix III. 7.

\subsubsection{Energy Dependence of the Detector Signal Ratios}

Having obtained expressions for all relevant terms in expression (4.2), the integration over photon energy was carried out for the various thicknesses of absorber, $\Delta \mathbf{x}_{i}$, for energies in the range of $100 \mathrm{keV}$ to $1000 \mathrm{keV}$. The ratios of the various integrals (each directly proportional to the photomultiplier output current signal for a given energy and absorber arrangement) were obtained for different thicknesses of absorber and plotted as a function of photon energy. The results of these computations appear in Figure (4-17). A listing of the normalized integration data used in this set of plots appear in Appendix IV. A listing of the plot coordinates themselves appears in Appendix III.4. Inspection of Figure (4-17) shows a strong energy dependence on the signal ratios for energies below $400 \mathrm{keV}$. As one proceeds to higher electron energy, we find a decrease in ratio sensitivity to photon energies. From the plots, therefore, the energy range of practical applicability of this technique would appear to be in the range of $200-700 \mathrm{keV}$. 


\section{FIGURE $4-17$}

Families of curves depicting NaI(TI) detector signal ratios as a function of primary electron energy parameterized to the various thicknesses of absorber used in the experiment. 


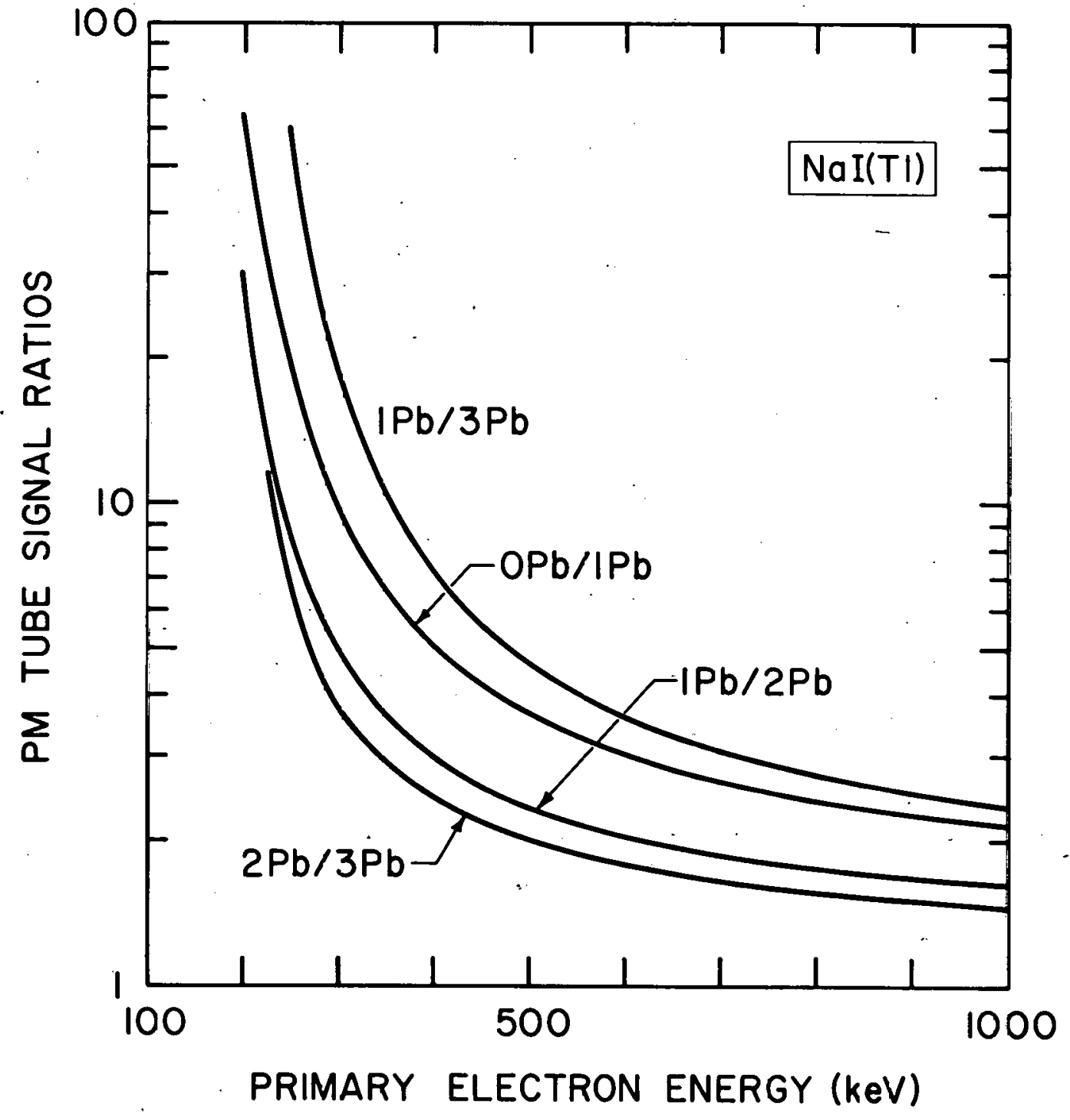

FIGURE 4-17 


\subsubsection{Code Error Analys is}

A general error analysis of the diagnostic plot of Figure (4-17) proceeds by considering the errors in the individual factors which contribute to the integral expression (4.2) for the photomultiplier tube current as a function of incident primary electron energy. Basically, the ratio of detector signals may be expressed as

$$
R=\frac{A_{1}+A_{2}+\cdots+A_{N}}{A_{1}^{\prime}+A_{2}^{\prime}+\cdots+A_{N}^{\prime}}
$$

where $A_{i}$ and $A_{i}^{\prime}$ are the areas under the integrand of Equation (4.2) in the $i^{\text {th }}$ electron energy interval for different thicknesses of absorber. Each of these area increments has a percentage uncertainty $\delta_{i}^{k}$ (or $\delta_{i}^{k_{1}}$ ) associated with the $k^{\text {th }}$ source of error, so that it may be rewritten as $A_{i}\left(1 \pm \delta_{i}^{k}\right)$ (or $\left.A_{i}^{\prime}\left(1 \pm \delta_{i}^{k}\right)\right)$. Introducing these expressions into Equation (4.7) and expanding results in

$$
\delta R_{i}^{k}= \pm \alpha_{i} \delta_{i}^{k} \mp \beta_{i} \delta_{i}^{k}
$$

where

$$
\alpha_{i}=\frac{A_{1}}{\sum_{i} A_{i}^{\prime}}
$$

and 


$$
\beta_{i}=\frac{\sum A_{i}}{\left(\sum_{i} A_{i}^{\prime}\right)^{2}}
$$

Therefore, the overall uncertainty in the magnitude of $(4.7)$ is dependent upon the correlation between $\delta_{i}^{k}$ and $\delta_{i}^{k_{1}}$. For the various factors in Equation (4.2), the most reasonable correlations appeared to be the following:

(i) $\delta_{i}^{k}=\delta_{i}^{k_{1}}$ for the uncertainties in detector efficiencies, Bremsstrahlung spectra statistics, and systematic Bremsstrahlung errors

(ii) $\delta_{i}^{k}=\left(\Delta / \Delta^{\prime}\right) \delta_{i}^{k}$ for the uncertainties in transmission coefficients for two absorbing slabs of thickness $\Delta$ and $\Delta^{\prime}$.

Furthermore, the following correlations were assumed between $\delta_{i}^{k}$ and $\delta_{j}^{k}$ (or between $\delta_{i}^{k}$, and $\left.\delta_{j}^{k}{ }^{\prime}\right)$ :

(i) Statistical fluctuations in the Bremsstrahlung spectra were treated as independent uncertainties.

(ii) The systematic error in the Bremsstrahlung spectral distribution was presumed to be \pm 58 at the lowest energies and $\mp 5 \%$ at cut-off. 88

(iii) The uncertainties in the detection efficiency 
were presumed to be $0 \%$ at $100 \mathrm{keV}$, rising to \pm 108 at $1 \mathrm{MeV}$.

(iv) The relative absorption cross-section errors in the transmission coefficients were presumed to be $\delta \sigma / \sigma= \pm 28$ at $100 \mathrm{keV}$ and $\delta \sigma / \sigma=\mp 2 z$ at cut-off.

Under these assumptions, the following set of contributing partial uncertainties results for primary electron energies of $300 \mathrm{keV}$ and $700 \mathrm{keV}$ and the ratio of $1 \mathrm{~Pb} / 2 \mathrm{~Pb}$ absorbers:

\section{$1 \mathrm{~Pb} / 2 \mathrm{~Pb}$}

$$
\begin{aligned}
& \delta \mathrm{R}^{\mathrm{k}} / \mathrm{R}^{\mathrm{k}} \\
& \delta \mathrm{R}^{\mathrm{k}} / \mathrm{R}^{\mathrm{k}} \\
& \left(E_{0}=300 \mathrm{keV}\right) \\
& \left(E_{0}=700 \mathrm{keV}\right)
\end{aligned}
$$

1. Bremsstrahlung

Spectra

Fluctuations

2. Bremsstrahlung

0.28

0.78

Spectra

Systematic

Error

3. Detector Efficiency

2.58

$-0.28$

4. Transmission

Coefficients

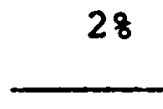

3.58
0.28

18

Overall Uncertainty

Thus, from the above estimates, we see that the 
uncertainty in the plots $(4-17)$ is of the order-of 1-48. If, as a "worst case" assumption, the " $i$ " contributions to the $k^{\text {th }}$ error source are presumed to be independent, the above overall uncertainty rises to $\sim 5 \%$ and $\sim 28$ for $300 \mathrm{keV}$ and $700 \mathrm{kev}$, respectively. Also, similar calculations for the $1 \mathrm{~Pb} / 3 \mathrm{~Pb}$ absorption ratio indicate that these overall uncertainties will rise to $\rightarrow 78$ and

28 for $300 \mathrm{keV}$ and $700 \mathrm{keV}$, respectively. As shall be discussed in Section 4.7, these errors are considerably less than the experimental uncertainties in determining absolute energy values.

\subsubsection{Energy Resolution}

To test the energy resolving power of the absorption spectroscopy technique used here, an electron energy distribution function consisting of a double-delta function,

$$
E(E)=\frac{1}{2} \delta\left(E_{1}-E_{0}+\delta\right)+\frac{1}{2} \delta\left(E_{1}-E_{0}-\delta\right),(4.10)
$$

was introduced computationally, with the "center energy", $E_{0}$, equal to $500 \mathrm{keV}$. Assuming equal electron fluences impinging onto the target at the two energies $E_{1}$ and $E_{2}$, one may express the detector signal ratio to be expected as 


$$
R=\frac{I_{1}\left(E_{1}\right)+I_{1}\left(E_{2}\right)}{I_{2}\left(E_{1}\right)+I_{2}\left(E_{2}\right)}
$$

where $I_{1}$ and $I_{2}$ denote the signal amplitudes from the detectors for the two components of electron energy in the primary beam.

Since the angular spread of the electron trajectories is not known in our experiment, two limiting cases of electron trajectories striking the target were considered:

(i) A monodirectional electron beam with no angular divergence, and

(ii) a "diffuse" electron beam whose particles and radiation pattern satisfy the condition that

$$
\left\langle\theta_{\mathrm{Oe}}^{\prime}\right\rangle\langle\rangle\left\langle\theta_{\mathrm{or}}^{\prime}\right\rangle
$$

Examples of the Bremsstrahlung radiation pattern for case (i) were shown in Figures $(4-5)-(4-13)$ where, predictably, the FWHM of the radiation pattern was found to decrease sharply with increasing electron energy. For this situation, assuming that the intensity distribution functions are roughly Gaussian profiles with respect to angle, the forward-directed intensity to the detectors $\left(\theta_{\text {or }}^{\prime} \sim 0^{\circ}\right)$ is related to the total integrated 
radiation intensity (over the forward $2 \pi$ hemisphere) by $I_{\theta} \sim 0^{\circ} \sim I_{\text {TOTAL }} /<\theta_{\gamma \text { FWHM }}>2$. Thus, the integrated radiation intensities for the different electron energies were.weighted according to the relative size of the square of the FWHM angular widths in computing the ratios of detected radiation signals in Equation (4.11). In the case of the diffuse beam, the radiation pattern will be isotropically distributed as a function of, angle and electron energy, and one may use the forward $2 \pi$-integrated intensity spectra directly in Equation (4.11), as in Section $(4.4,2.2)$.

The ratios in Equation (4.11) were computed for the four combinations of thicknesses of absorber in the range $0 \leq \delta \leq 200 \mathrm{keV}$ for both types of electron beams. Figure $(4-17)$ was then used to predict the ring electron energy for each of these combinations. The results of the computations are shown in Figure (4-18).

Inspection of Figure (4-18) shows the resolving power of the measurement to be fairly insensitive to spreads in the electron energy distribution. The "measured" average energies were always larger than the actual average energy, $E_{0}$, with the differences between these two energies being different for the various thicknesses of absorber. For example, for the diffuse 
FIGURE $4-18$

Primary electron energy as predicted by various NaI(T1) detector signal ratios having different absorber combinations for a mono-directional and diffuse electron beam. 


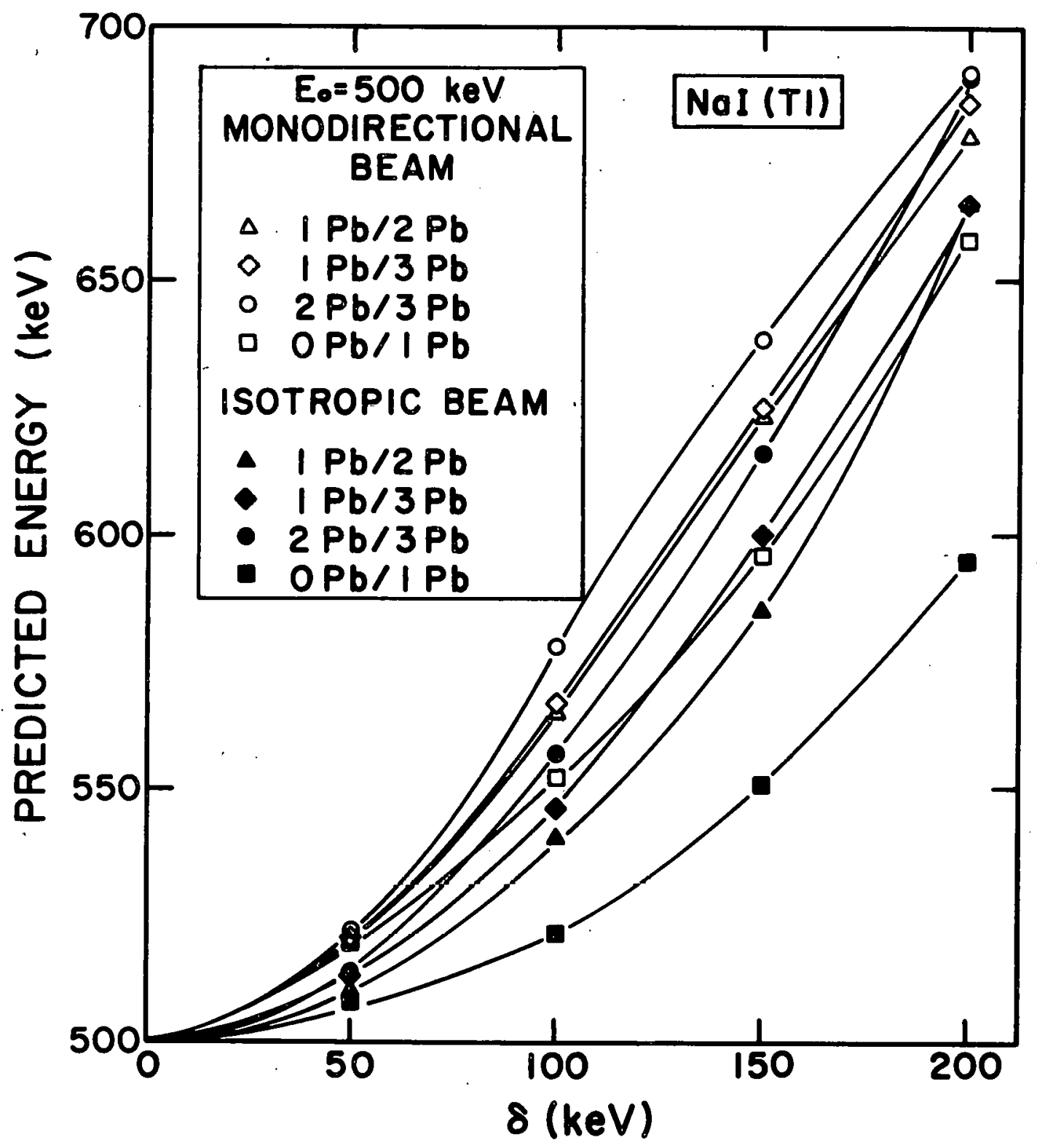


beam with $\mathrm{E}_{1}=300 \mathrm{keV}$ and $\mathrm{E}_{2}=700 \mathrm{keV}$, the $0 \mathrm{~Pb} / 1 \mathrm{~Pb}$ ratio predicts $600 \mathrm{keV}$, where the $2 \mathrm{~Pb} / 3 \mathrm{~Pb}$ ratio predicts $700 \mathrm{kev}, \underline{i}$. e.., a predicted energy spread of only $100 \mathrm{keV}$, or about 258 of the "actual" energy spread of $400 \mathrm{keV}, \underline{i} . \underline{e} .$, there is a factor of about four between the apparent and the actual energy spread in this case. In the case of the unidirectional beam under the same conditions, the $0 \mathrm{~Pb} / 1 \mathrm{~Pb}$ ratio predicts $660 \mathrm{keV}$, where the $2 \mathrm{~Pb} / 3 \mathrm{~Pb}$ ratio--again--predicts $700 \mathrm{keV}$, $\underline{\text { i.e. }}$., a predicted energy spread of only $40 \mathrm{keV}$, or about $10 \%$ of the "actual" energy spread.

It follows that these two particular absorber ratios should predict the greatest energy spread, since they sample such different portions of the $X$-ray spectrum. Also, as the energy spread increases, one notices a distinct trend in the energy spread curves towards the higher energy in the double-delta function distribution. This feature simply indicates the increasing importance of the high energy component of the $\mathrm{x}$-ray signal relative to the lower energy $\mathrm{X}$-rays. This trend is accentuated for the unidirectional electron beam, where the radiation pattern peaks sharply in the forward directions for the higher electron energies. 


\subsection{Experimental Procedures}

Prior to, and during, the energy measurement experimental runs, a number of alignment, collimation, and detector calibration tests were performed. These are considered in detail in the following sections.

\subsubsection{Alignment and Collimation Tests}

Before the shielded $\mathrm{X}$-ray detector system was placed next to Berta, tests were made to insure that all three collimators "saw" the same geometric area in the object plane of the wire target. This was done using a point light source located behind the lead coliimators--where each of the three detectors would normally reside--and a screen placed $46 \mathrm{~cm}$ in front of the collimators. The three collimators were measured to "view" the same $8.3 \mathrm{~cm}$ by $8.8 \mathrm{~cm}$ object plane area to within $1 \mathrm{~mm}$ in boundary measurement accuracy. Positioning and alignment of the detector system were fairly tedious propositions owing to the bulk of the elements of the system to be aligned. A $5 \mathrm{~mW}$ HeliumNeon laser mounted inside the lead collimator housing was used first to align the center collimator with the radiator as it hung in position inside Berta. Tests were then made to insure that the radiator re-positioned itself accurately after it was furled and unfurled from 
the suspending glass rod (which it did). This was an operation which would have to be repeated often during the experiment to perform "null tests". This alignment procedure was repeated every time the monitor (and/or Berta) was (were) moved for reasons such as diode foil changes, etc. Also, tests were made to check that the detector system repositioned itself properly each time it was rolled away from Berta on its tracks.

As part of the measurements, it was desired to place the target at different axial positions in the layer. However, owing to the cumbersome nature of the alignment procedure, it turned out to be far easier-rather than move the target axially and then have to re-align the collimators--to move the electron rings themselves, leaving the radiator/collimators fixed in position. This was easily accomplished by moving the downstream mirror $5 \mathrm{~cm}$ and thereby relocating the minimum of the magnetic mirror well.

\subsubsection{Detector Calibration}

A linear dynode chain voltage division ratio was used in biassing the photomultiplier tubes, with $\mathrm{R}_{c}=$ $47 \mathrm{k} \Omega$. The last four stages were also capacitively loaded to handle possible current surges, with $c_{7,8}=$ $0.005 \mu \mathrm{fd}$ and $c_{9,10}=0.01 \mu \mathrm{fd}$. Throughout the 
experimental runs, the measured detector signals did not exceed $300 \mathrm{mV}$. With an emitter follower gain of 0.6 and a resistive load on the photomultiplier tube of $1 \mathrm{k} \Omega$, this represents a peak detector current of $-5 \times 10^{-4} \mathrm{~A}$, about $25 \%$ of the direct current rating of $2 \times 10^{-3} \mathrm{~A}$ for this particular tube. ${ }^{89}$ Even if this peak current level were sustained for $20 \mathrm{\mu sec}$, it would represent only a 28 voltage decrease on the last dynode ballast capacitor. Hence, the photomultiplier tubes were always operated well within the conditions necessary for linear performance.

Diagnostic tests were performed to check on the linearity of the overall detector response to photocaptured $\mathrm{x}$-rays in the energy range of $0.1-1.3 \mathrm{MeV}$. Measurements similar to those described in Section 3.2.3.3 were made on each detector. In this case, however, the sodium iodide crystal, in contrast to the Pilot B ingot, possesses a significant photoabsorption cross-section in this energy range (which accounts for its increased detection efficiency in these energy ranges). Thus, it is possible to make a direct medsurement nf $\mathrm{x}$-ray "line radiation" from the radioactive calibration sources used. The results of these measurements are shown in Figure (4-19). We see that each of the three detectors 
$-226-$

FIGURE $4-19$

X-ray energy calibration of NaI(TI) X-ray detectors. 


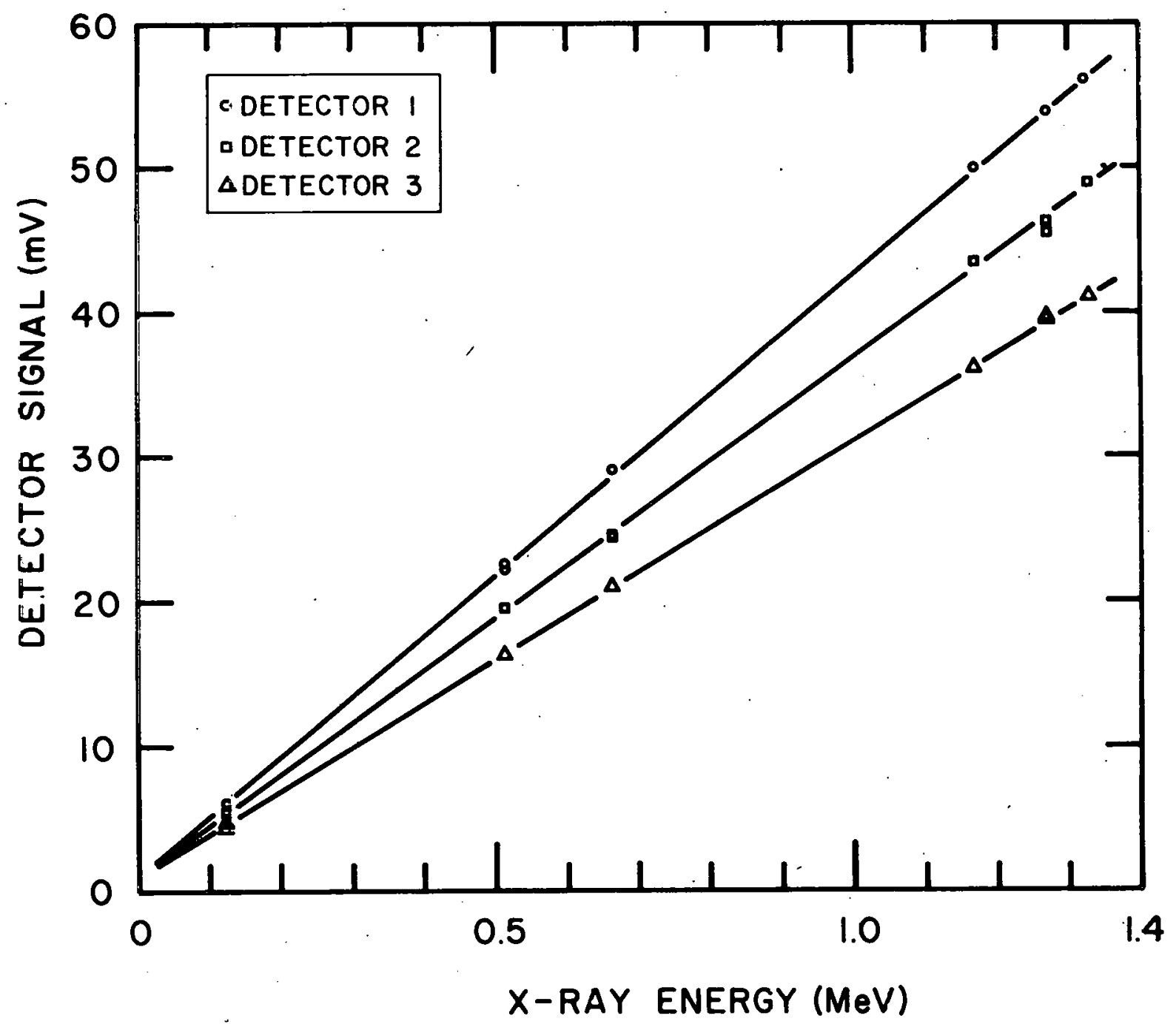


is quite linear in energy response over the range examined. In addition, the detectors are within $20 \%$ of one another in absolute response characteristics, a small difference easily explained by dissimilarities in photomultiplier tubes as well as differences in resistances used to construct the dynode chain high voltage circuits. For the sake of illustration, Figure (4-20) shows two sample traces of uniform detector irradiation by $\mathrm{CO}^{60}$ and $\mathrm{Na}^{22}$. The photographs represent six-second exposure times. Clearly visible in the traces are the two gamma lines of cobalt ( 1.17 and $1.33 \mathrm{MeV})$, as are the $1.274 \mathrm{MeV}$ and $0.511 \mathrm{MeV}$ (annhilation radiation) lines of $\mathrm{Na}^{22}$. In addition, the compton "edge" energies, as well as the $180^{\circ}$ backscatter lines (the height of which on the trace should equal the distance from a Compton edge to its correspondent photoabsorbed $\mathrm{x}$-ray linel. Such traces were used in the absolute calibration of the detectors, a topic discussed in Appendix III.7. Each photomultiplier tube was equipped with an independently variable set of taps from the main high voltage power supply. At the outset of the experiment; these voltages were adjusted to give approximately equal signals from all three detectors with "l", "2", and "3" lead absorbers in front of the collimators. 
$-229-$

FIGURE 4-20

$\mathrm{NaI}(\mathrm{Tl})$ detector response to $\mathrm{Co}^{60}$ and $\mathrm{Na}^{22}$. 

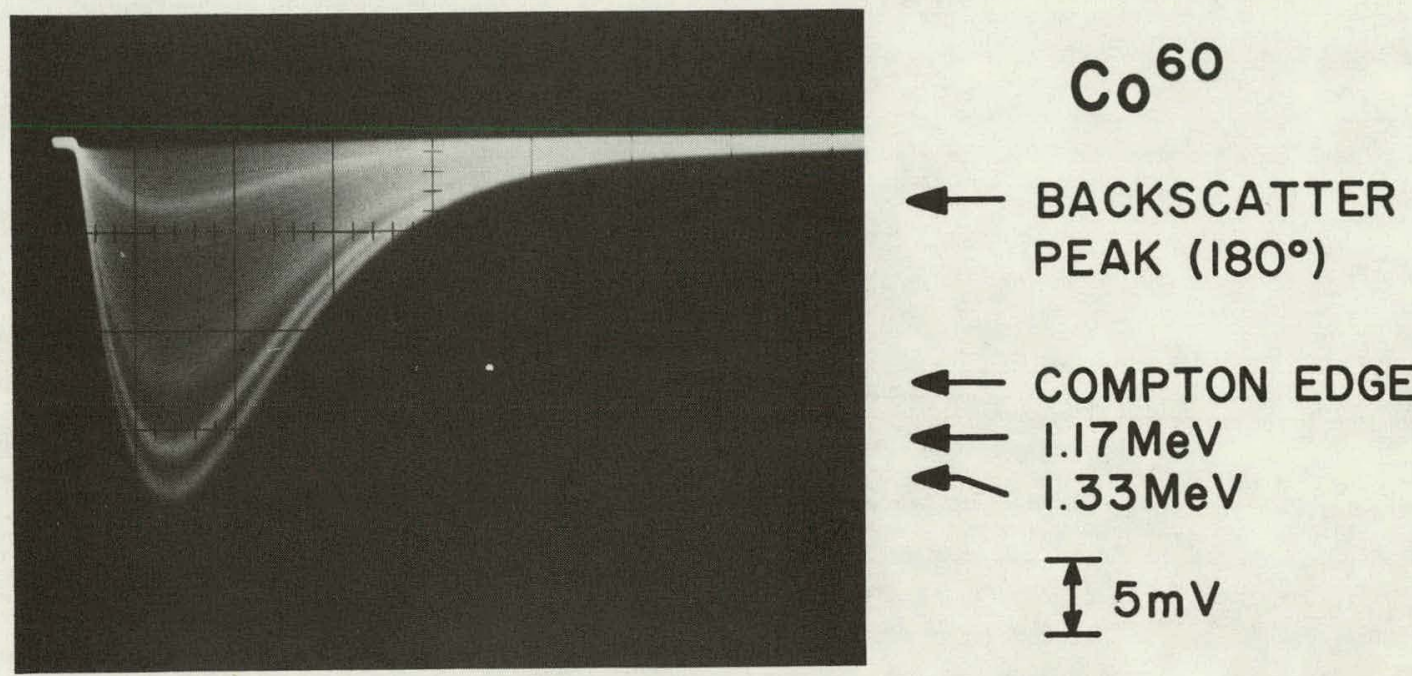

\section{— COMPTON EDGE \\ $\leftarrow 1.17 \mathrm{MeV}$ \\ - $1.33 \mathrm{MeV}$ \\ I $5 \mathrm{mV}$

$$
V_{P M T}=-900 \mathrm{~V}
$$

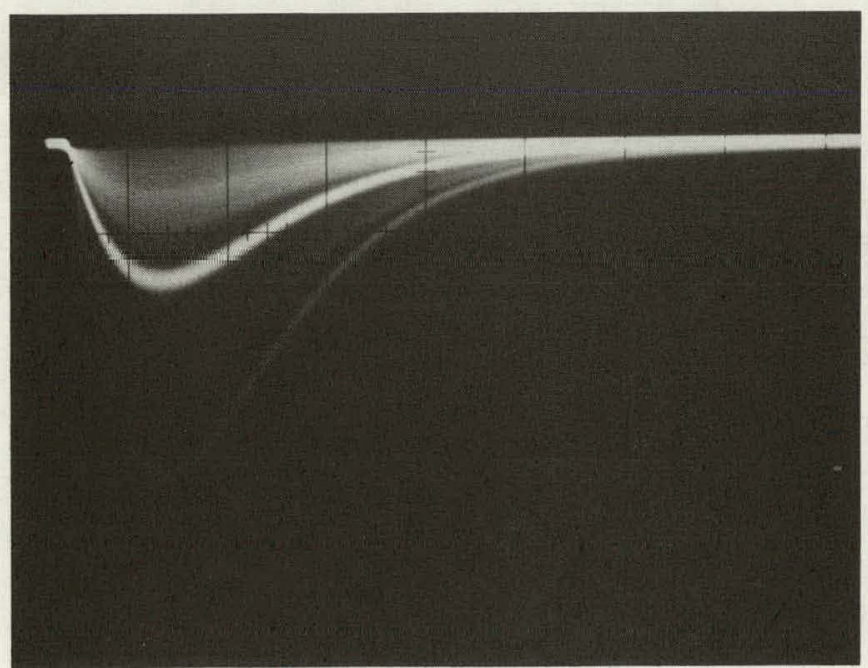

$$
\mathrm{Na}^{22}
$$

\section{BACKSCATTER}

$\longleftarrow$ PEAK $\left(180^{\circ}\right)$

COMPTON EDGE $0.511 \mathrm{MeV}$

$\leftarrow 1.217 \mathrm{MeV}$

$\pm 5 m V$

$V_{P M T}=-900 \mathrm{~V}$

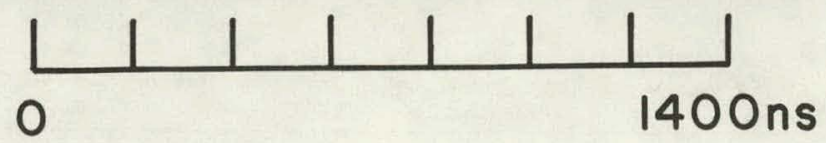


The relative detector calibrations were checked regularly by placing equal absorber thicknesses in front of all three detector collimators and, varying the thicknesses of all absorbers from shot-to-shot, measuring the target Bremsstrahlung intensities from the three detectors. Samples of the calibration data obtained in this way are shown in Figure (4-21). To avoid long-term systematic drifts (notably the electron beam diode voltage, photomultiplier gains, etc.), it was desired to perform the overall experimental runs in as short a time as possible (the total run time was 35 continuous hours). Overall, the relative calibration drifts were found to be about 5:--limited largely by the accuracy with which they could be measured.

A fundamental question in the interpretation of the detector signals was the possible role of background $\mathrm{X}$-radiation resulting from the scattering of the fast electrons from parts of the experimental structures exclusive of the tungsten target. To this end, a number of "wire-in/wire-out" checks were undertaken at the outset of the experimental runs and during the above-mentioned oalibration checks to insure that the collimation to the detectors was, in fact, effective in eliminating such spurious hard ( $500 \mathrm{keV})$ radiation. Samples of the 
$\mathrm{X}$-ray detector response with tungsten wire target suspended in, and removed from, the ring confinement region. 
"WIRE OUT"

PM I

PM 2

PM 3

E-LAYER B PROBES
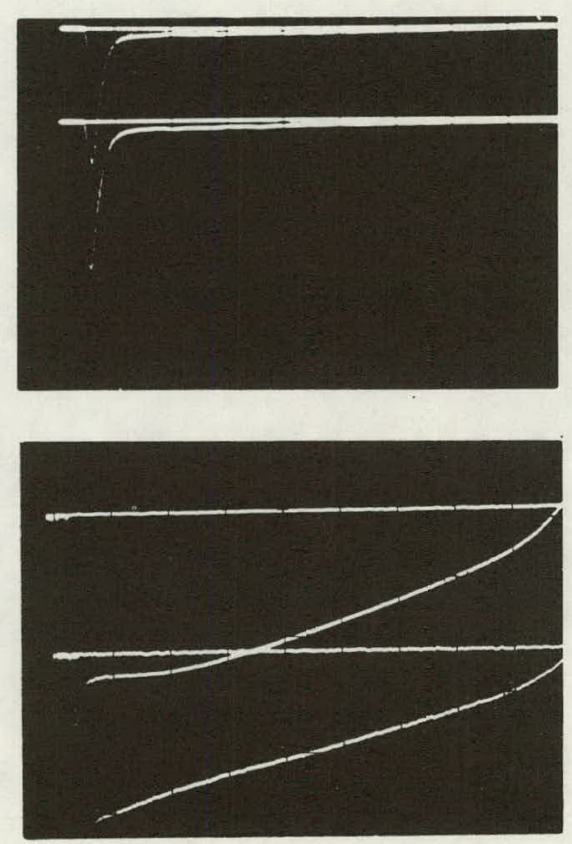

\section{CALIBRATION TESTS}

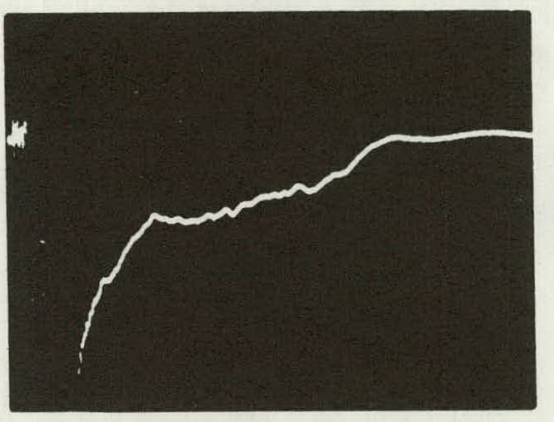

[O.IV: "IPb"

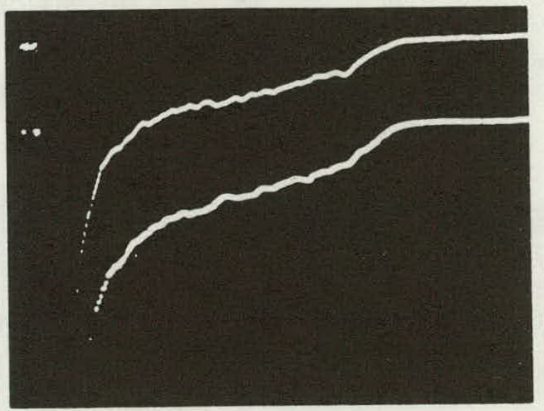

[ O.IV: "IPb"

[ O.IV: "IPb"

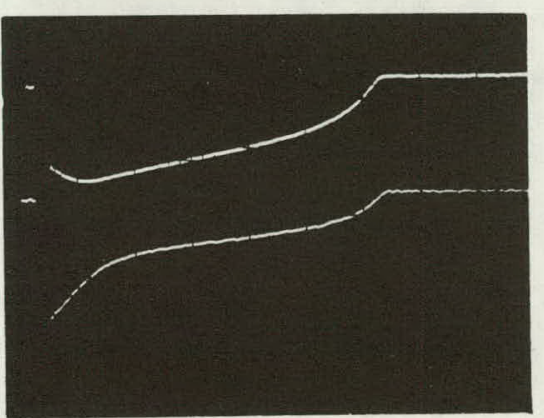

$\left[\begin{array}{cc}0 & -108 \mathrm{~cm} \\ 110 \mathrm{G} & -116 \mathrm{~cm}\end{array}\right.$ SWEEPS: $2 \mu \mathrm{s} / \mathrm{div}$ 
detector signals conly the tin absorber was positioned in front of the collimators) with the wire target removed are shown in Figure (4-21). These are to be contrasted with the signals obtained with an electron ring of similar strength with the target lowered into position: removing the target is seen to reduce the received $x$-ray detector signals by over a factor of 20 .

It was also necessary to verify experimentally the presumption that, owing to the $\pm 7^{\circ}$ angular separation of the detectors (which ought to be well within the $70^{\circ}$ radiation pattern spread, as mentioned in Section 4.4.2.2), all detectors indeed observed the same Bremsstrahlung spectrum. This was checked by permuting the various thicknesses of lead absorbers among the different collimators during the experimental runs as standard procedure. This allowed the different detectors to "view" different portions of the $\mathrm{X}$-ray spectrum. Furthermore, the detectors themselves were interchanged amongst the various collimator channel locations to check for. systematic errors. Comparison of the results of these permutations on a shot-toshot basis showed that the various signal ratios for different thicknesses of absorber were independent of the position of the absorber/detector within the accuracy of the measurement. 
4.6 Average Ring Electron Energy Measurements

Due to the perturbing effect of the wire target on the electron rings, two sets of experimental energy measurements were performed. First, the energy loss rate of the electrons due to collisions with the background gas was varied by changing both the density (pressure) and type of the backfill gas used. Second, comparison measurements were made in which the machine parameters were held constant, and the effect of the target perturbation on the ring was varied.

\subsubsection{Variation of Machine Parameters}

To study the effects of different electron collisional energy loss rates, energy measurements were made at two pressures of hydrogen ( 450 and 750 mTorr hydrogen, base pressure of 15 mTorr nitrogen). Measurements were also made using an admixture of hydrogen and argon. The choice of argon lay principally in its enhanced (by a factor of 50) 90 scattering of the electrons, but contributing (with the fractional pressures used) less than 108 to the particles' energy losses per unit length of distance travelled than did the hydrogen with which it was admixed. The effects of this enhanced loss rate will be discussed in greater detail later.

(It would have been interesting to have investigated 
the effect of different ring primary electron energies. However, it was quite difficult to vary the electron beam energy over a significant range with the existing beam equipment, owing largely to unreliable Marx performance below $30 \mathrm{kV} /$ capacitor, and frequent Blumlein switch misfires at low Marx erected voltages. In addition, practical considerations limit the maximum strength of the pulsed confining field applied to Bexta to about $200 \mathrm{G}$ and, hence, the maximum electron energy which may be confined in the magnetic field ( $500 \mathrm{kev})$. Therefore, a variation of the primary electron energy was pretty much out of the question.)

\subsubsection{Variation of Wire Perturbation}

The effect of the perturbation of the wire target was varied by placing it at different axial positions in the circulating current distribution. In the measurements performed, the wire was located at two positions: near the center of the current distribution ("center"), i.e.., a maximum perturbation; and at the edge (about $6 \mathrm{~cm}$ from the center) of the current distribution ("edge"), where it is a minimal perturbation. The location of the wire with respect to the electron ring magnetic field distribution at these two positions is shown in Figure (4-22). As was mentioned earlier, in 
FIGURE 4-22

Location of tungsten wire target with respect to the ring diamagnetic profile for the two target positions studied in this work. 

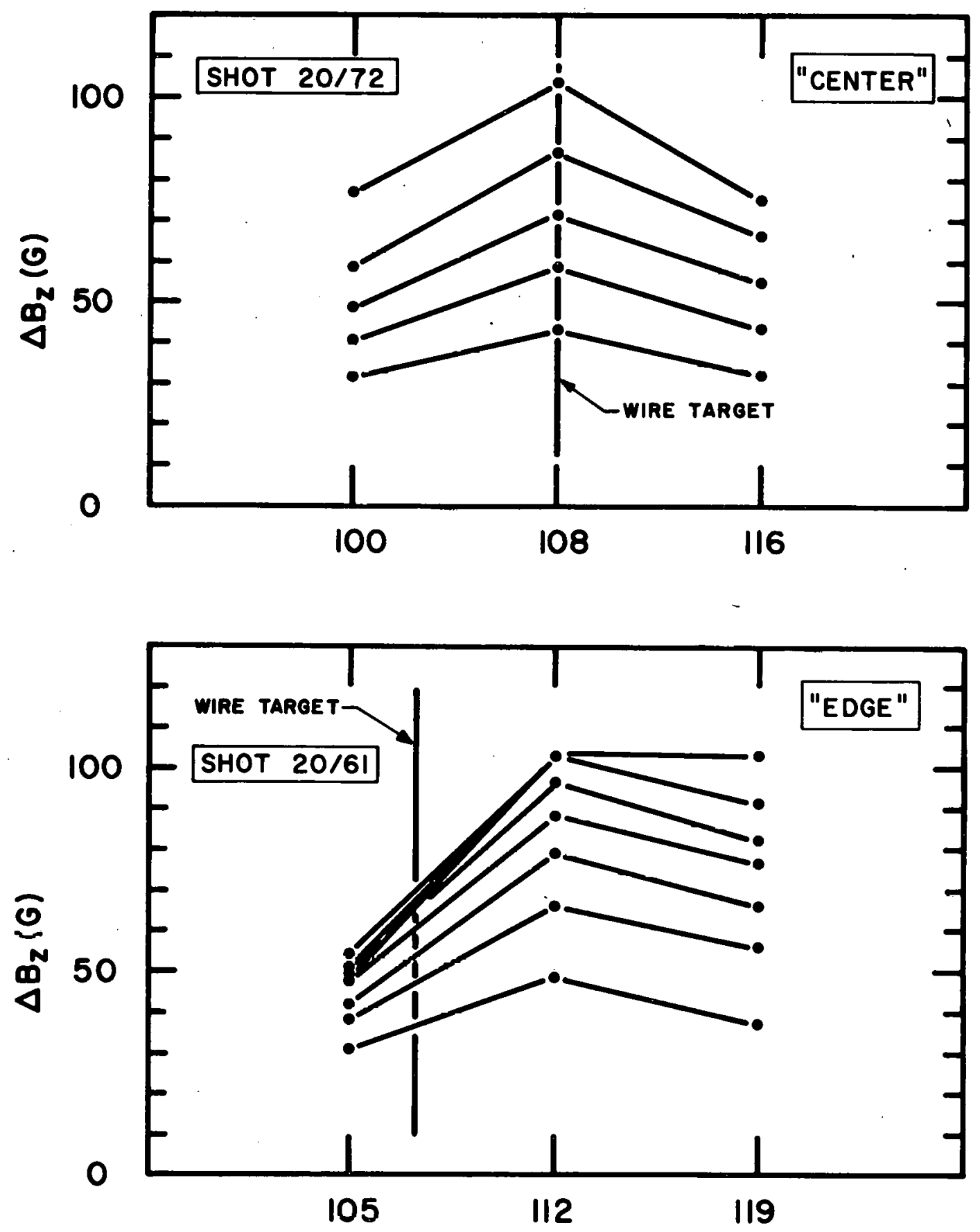

PROBE POSITION (cm FROM DOWNSTREAM END OF BERTA) 
practice it was easier to move the electron ring axially than to move the target; which is how the perturbing influence of the wire was varied.

\subsubsection{Summary of Experimental Results}

Samples of the experimental data obtained under the various conditions described above are shown in Figures (4-23) - (4-26). The first three oscillograph traces are signals received from each of the three Bremsstrahlung detectors (labelled "PMl"., "PM2", and "PM3" in the figures). The thickness of the lead absorber in front of each detector is indicated, as are the gains of the oscilloscope amplifiers. The fourth trace is the signal from the magnetic probe centered in the complement of three probes used to monitor the overall diamagnetic behaviour of the electron rings. The data shown in Figure (4-23) was obtained with the wire target located near the axial center of the electron ring ("center"). The data of Figure (4-24) was taken by moving the downstream mirror $5 \mathrm{~cm}$ further downstream, thereby moving the ring downstream so that the wire target probed the upstreain axial edge of the electron ring ("edge"). Characteristically, all of the $x$-ray traces strongly resemble the signals from the magnetic probes: an initial "setup" phase, a monotonic decay in time, and the final, 
FIGURE 4-23

Characteristic signals from three-channel $\mathrm{X}$-ray detector and axial magnetic probes for an electron ring confined in a backfill of 450 mTorr hydrogen; target located at axial center of ring current distribution. 

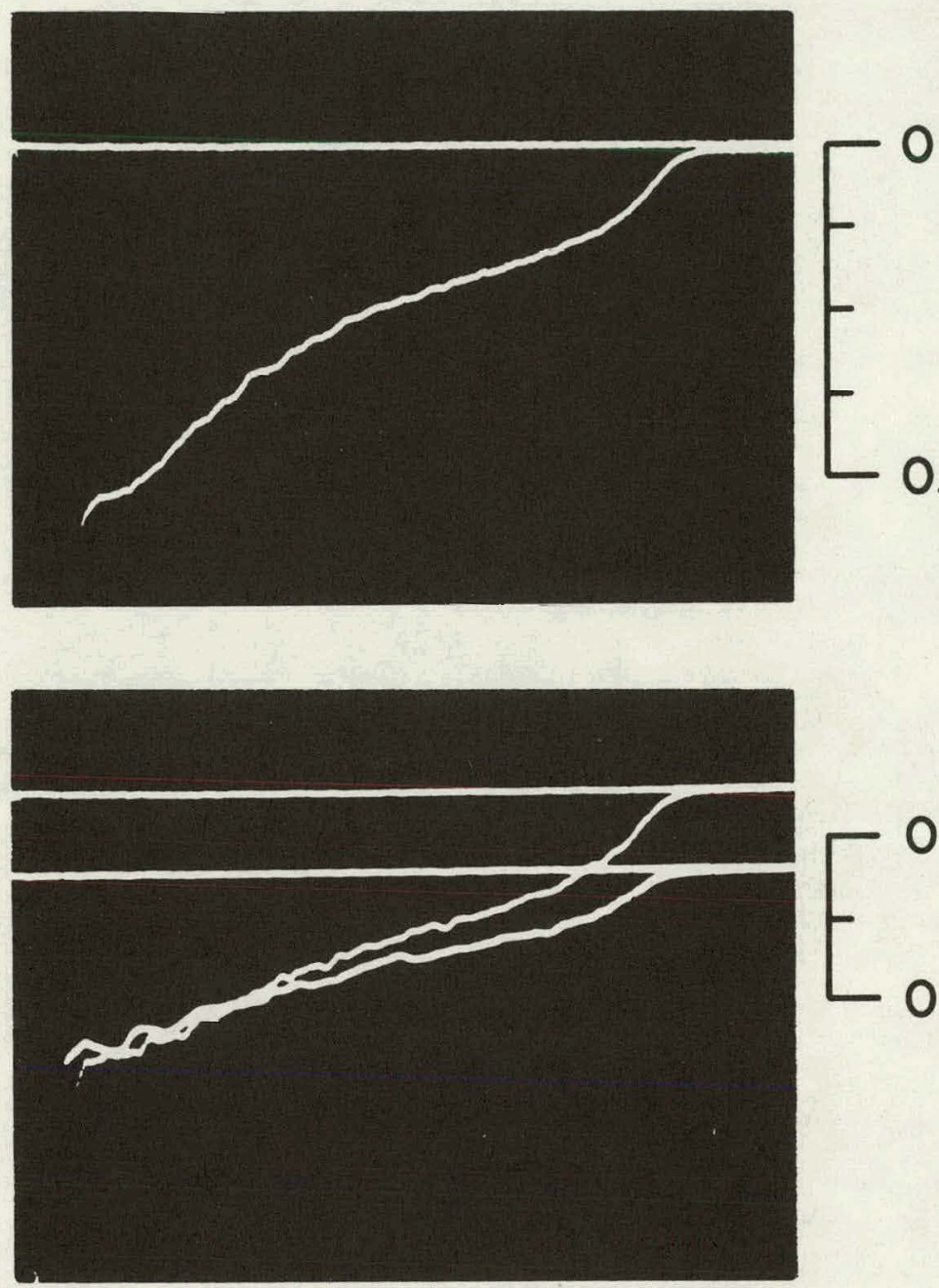

\section{HYDROGEN \\ $\mathrm{P}\left(\mathrm{H}_{2}\right)=450 \mathrm{mTORR}$ \\ ("CENTER") \\ SHOT 20/66}

$P M I: \mid P b$

$0.4 V$

0

$P M 3: 2 P b$

O.IV

PM2: 3Pb

PROBE 2

IIOG

SWEEPS: $2 \mu s / d i v$ 


\section{FIGURE 4-24}

Characteristic signals from three-channel $\mathrm{x}$-ray detector and axial magnetic probes for an electron ring confined in a backfill of 450 mTorr hydrogen; target located at edge of ring current distribution. 

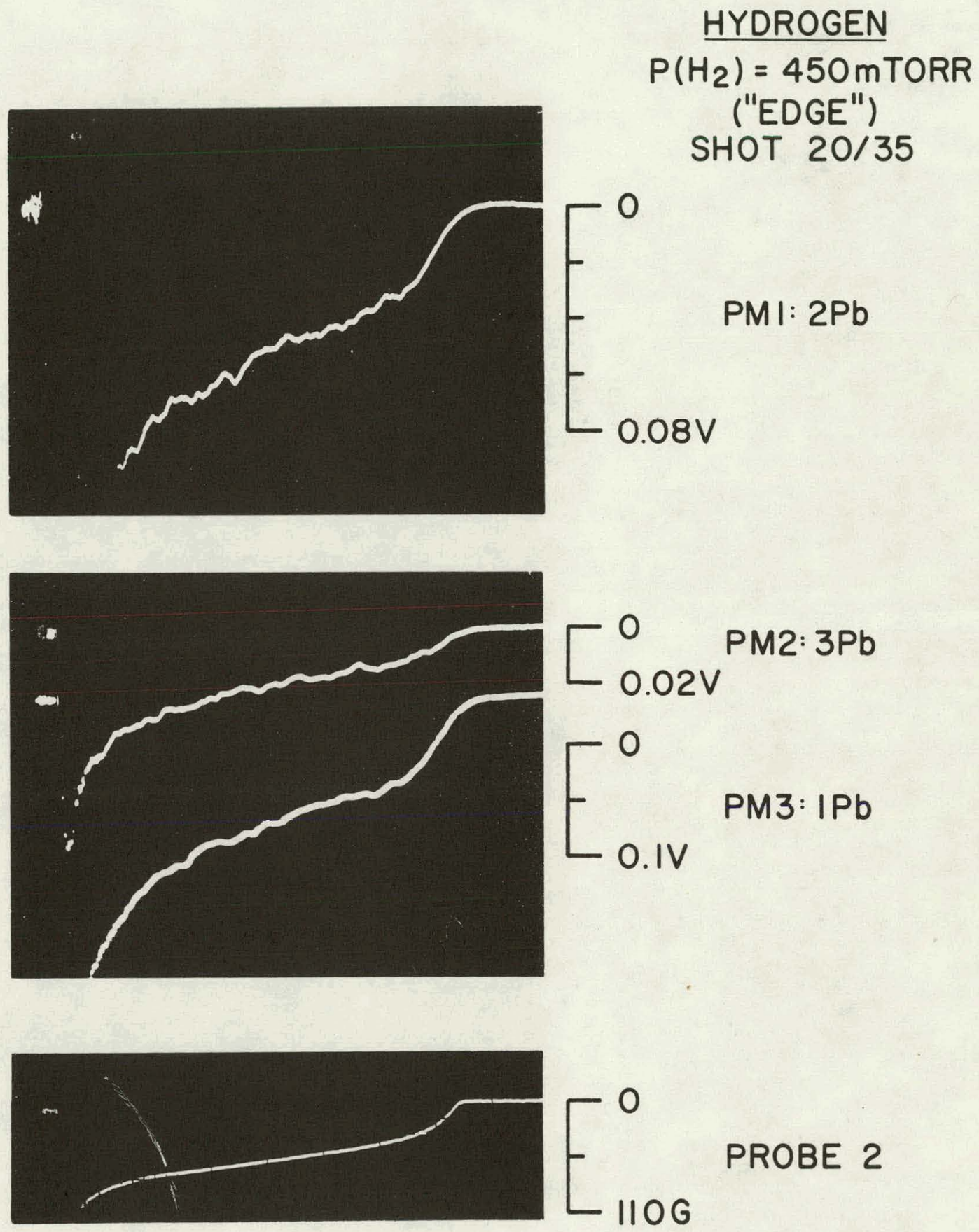

SWEEPS: $2 \mu \mathrm{s} / \mathrm{div}$ 
FIGURE $4-25$

Characteristic signals from three-channel $\mathrm{x}$-ray detector and axial magnetic probes for an electron ring confined in a backfill of 750 mTorr hydrogen; target located at edge of ring current distribution. 


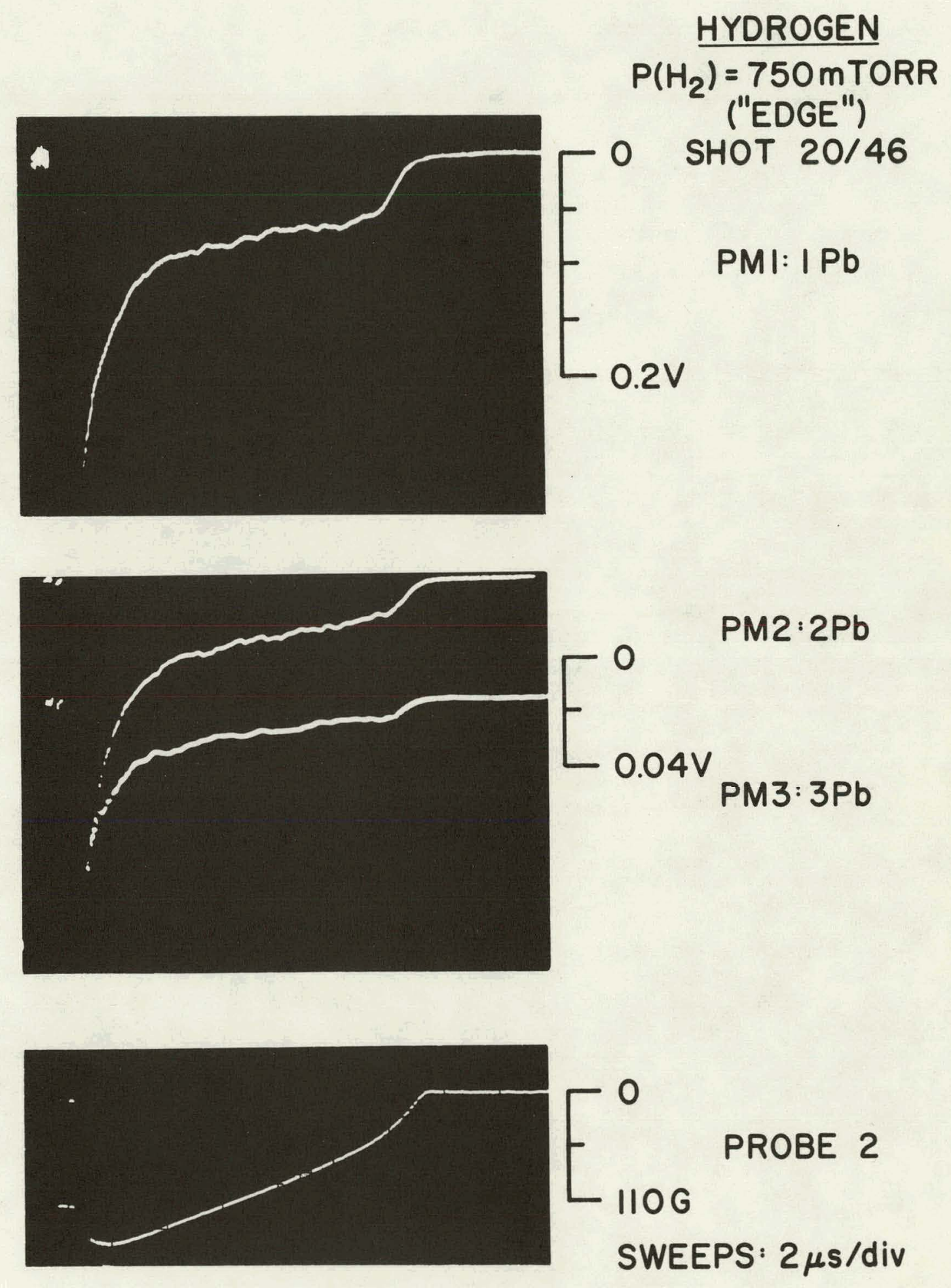

FIGURE 4-25 


\section{FIGURE $4-26$}

Characteristic signals from three-channel $\mathrm{X}$-ray detector and axial magnetic probes for an electron ring confined in a backfill of $410 \mathrm{mTorr}$ hydrogen and $6 \mathrm{mTorr}$ argon; target located at edge of ring current distribution. 


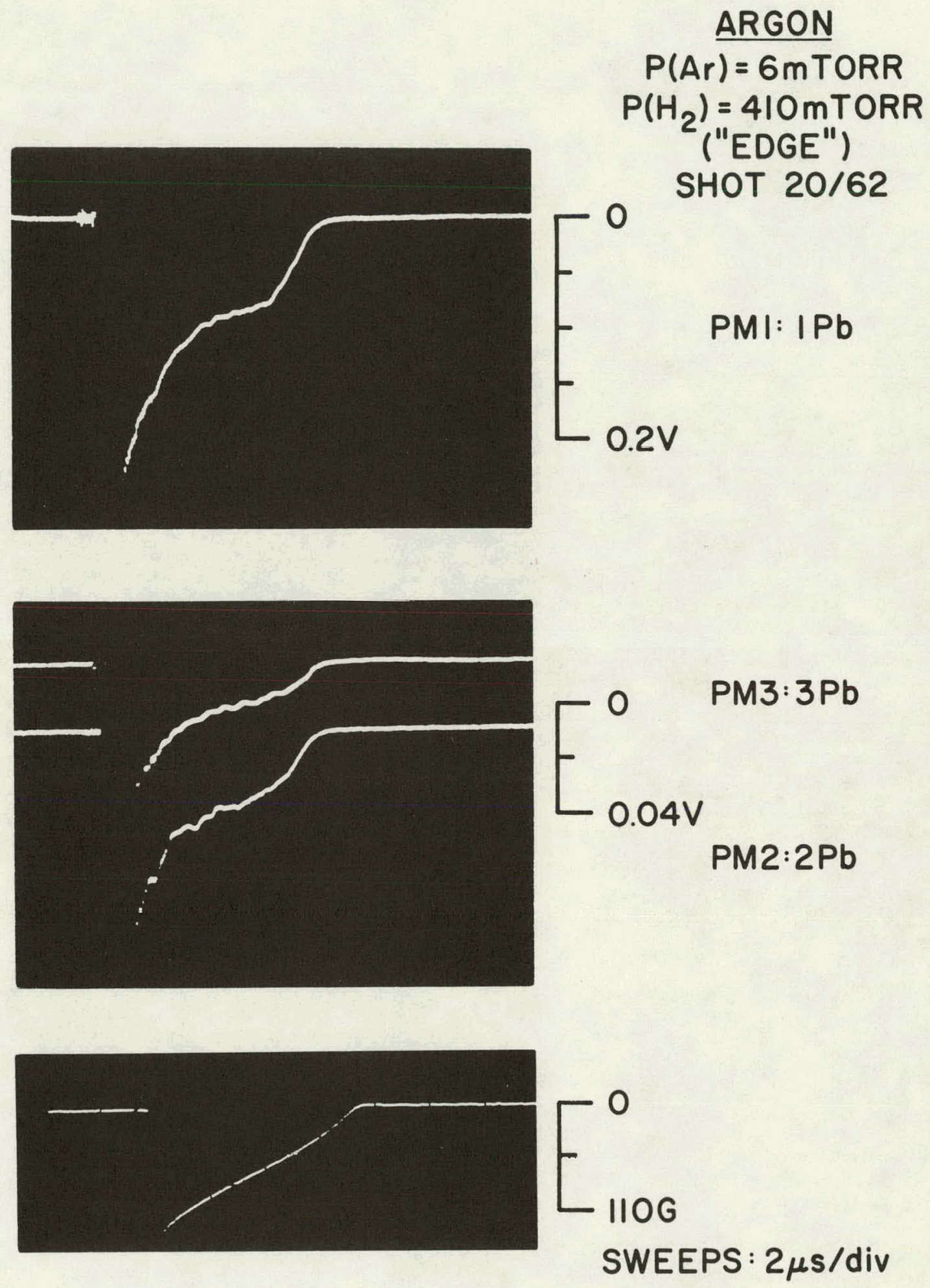


catastrophic "dump" are all in evidence in both sets of data. The non-existence-of an X-ray spike at the "dump" reconfirms the suppression, by a factor of $\geq 20$, of background radiation. However, one sees on the $\mathrm{X}$-ray signals evidence of stronger decay rates in the first 2-3 microseconds of confinement, followed by an interval in which the signals decay at a much lower rate (by a factor of $\sim 1 / 10-1 / 20$ ) which is fairly constant until the onset of the "dump". This behaviour is to be contrasted with the fairly even, linear signal decay observed on the magnetic probes which persists throughout the electron ring lifetime. The differences in characteristics between these sets of signals lie in the fact that the $x$-ray detectors are monitoring the high-energy electron current density distribution as a function of time (as discussed in Chapter 3), while the magnetic probes are sensitive only to the diamagnetic field generated by this current distribution. The strength of the magnetic field on axis for a cylindrically-symmetric current distribution is not strongly dependent on the axial length of the distribution when its axial length is comparable to its diameter. Thus, it is clear that significant changes in the details of the current density distribution could go almost "unnoticed" by axial magnetic field monitors which are all located 
less than the radius of the current distribution away from the axial center of that distribution.

Typically, the monitored detector signals ranged from $20 \mathrm{mV}$ to $150 \mathrm{mV}$ in the "quiescent" section of the signal after the initial transients have died away. Using data such as that shown in Figure (4-19), one may calculate (as in section 3.2.3.4) an expected percentage signal fluctuation under these conditions of between 38 and $7 \%$ based on the average number of photons (between $\approx 250 \mathrm{keV}$ and $500 \mathrm{keV}$ ) counted per $250 \mathrm{~ns}$ interval (between 200 and 1500 counts). These predictions are in keeping with the signal fluctuations to be observed in Figures (4-23) - (4-26). Also, due to inescapable pickup from the ringing of the Marx/Blumlein (with a period of 3-4 usec)--mainly on only one detector which, as a result, had only one lead absorber to increase its signal-to-noise--it was necessary to subtract low-level baseline oscillations from the detected signals. (These oscillations were typically 2-5 mv in amplitude which, for electron energies around $500 \mathrm{keV}$, could represent periodic energy fluctuations of 5-108:)

For a 508 field-reversing electron ring with the wire target located at the ring's axial center (cf. Figure 4-23)), the observed detector signals were 
typically $\sim 0.4-0.5 \mathrm{~V}(1 \mathrm{~Pb}), \sim 0.14-0.16(2 \mathrm{~Pb})$, and $\sim 0.10-0.12 \mathrm{~V}(3 \mathrm{~Pb})$. These signal levels corresponded, within a factor of two, with calculated values under these experimental conditions (this is discussed in detail in Appendix IV).

In order to obtain the final average ring electron energy as a function of time from traces such as. those shown in Figures $(4-23)-(4-26)$, the statistical fluctuations in the signals were averaged over a 500 ns time interval and the relative calibration factors described in Section 4.5 .2 were applied prior to taking the signal ratios. (Generally, the resulting intensity ratios $I_{0 \mathrm{~Pb}} / I_{2 \mathrm{~Pb}}$ and $I_{1 \mathrm{~Pb}} / I_{3 \mathrm{~Pb}}$ varied between $2.3-2.7$ and 4.0 - 5.0, respectively, from shot-to-shot. During the course of the decay of each electron ring, these ratios usually increased by about 10\%. (Unfortunately, the benefits of taking data with only the tin absorber in front of the collimators ("OPb") were not appreciated at the time the experimental runs were made, so no data was taken at that level of absorption.)

Using Figure (4-17), plots were then obtained of the average ring electron energy as a function of time for these different parameter studies. These plots appear in Figures $(4-27)-(4-30)$. 


\section{FIGURE 4-27}

Average electron energy as a function of time for an electron ring confined in a backfill of 450 mTorr hydrogen; target located at axial center of ring current distribution. 


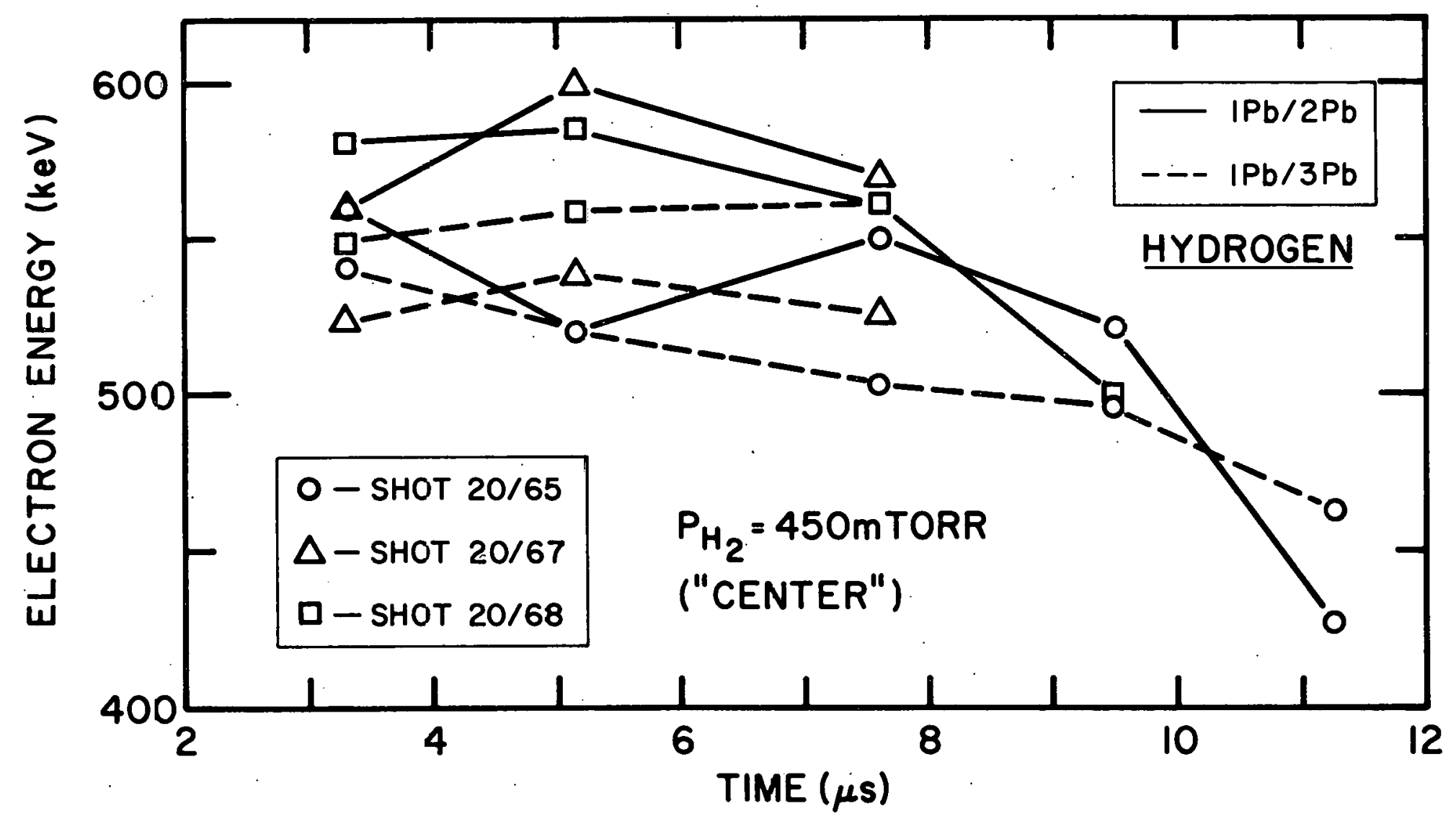

FIGURE 4-27 
FIGURE $4-28$

Average electron energy as a function of time for an electron ring confined in a backfill of 450 mTorr hydrogen; target located at edge of ring current distribution. 


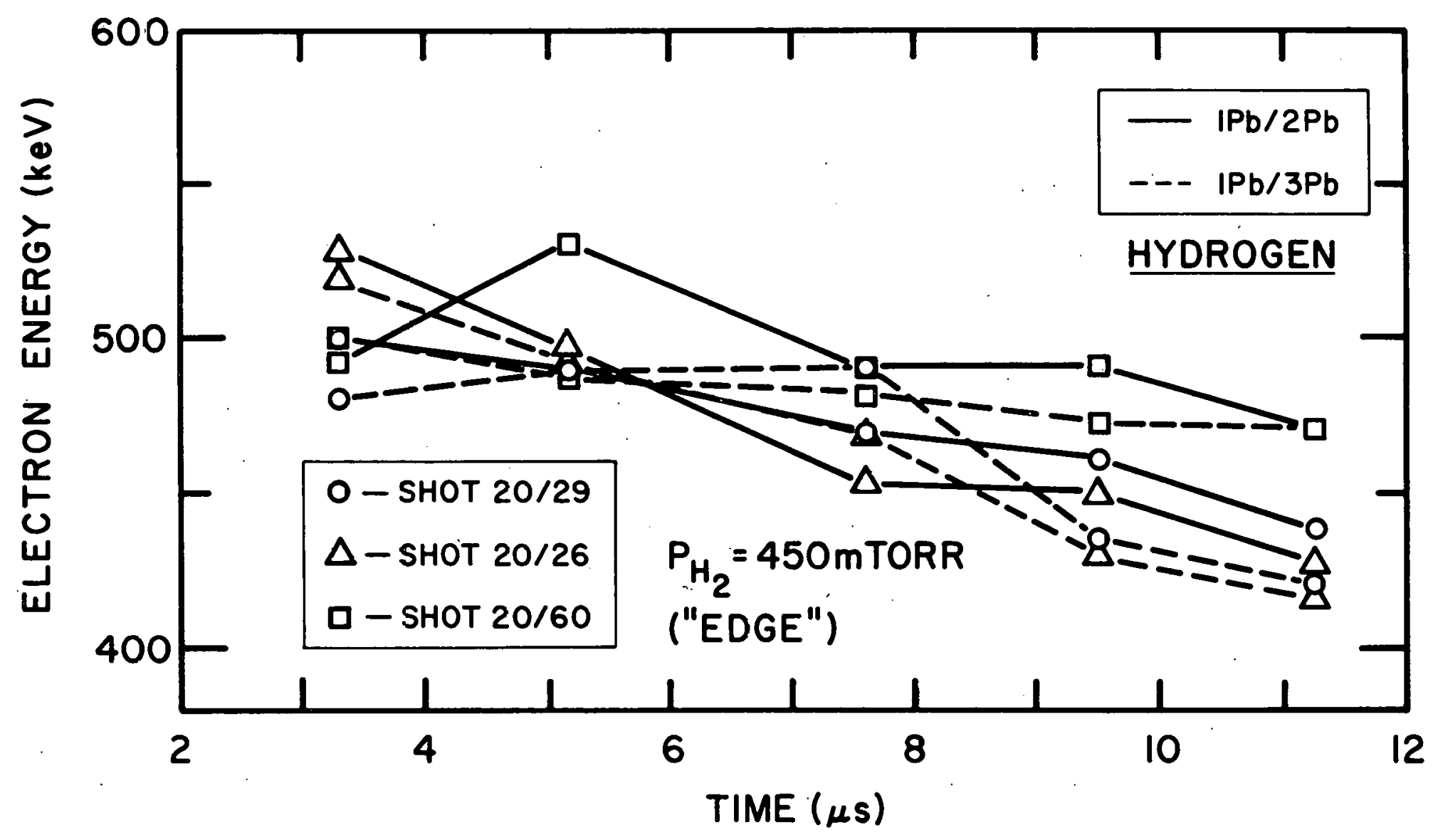


FIGURE $\quad 4-29$

Average electron energy as a function of time for an electron ring confined in a backfill of 750 mTorr hydrogen; target located at edge of ring current distribution. 


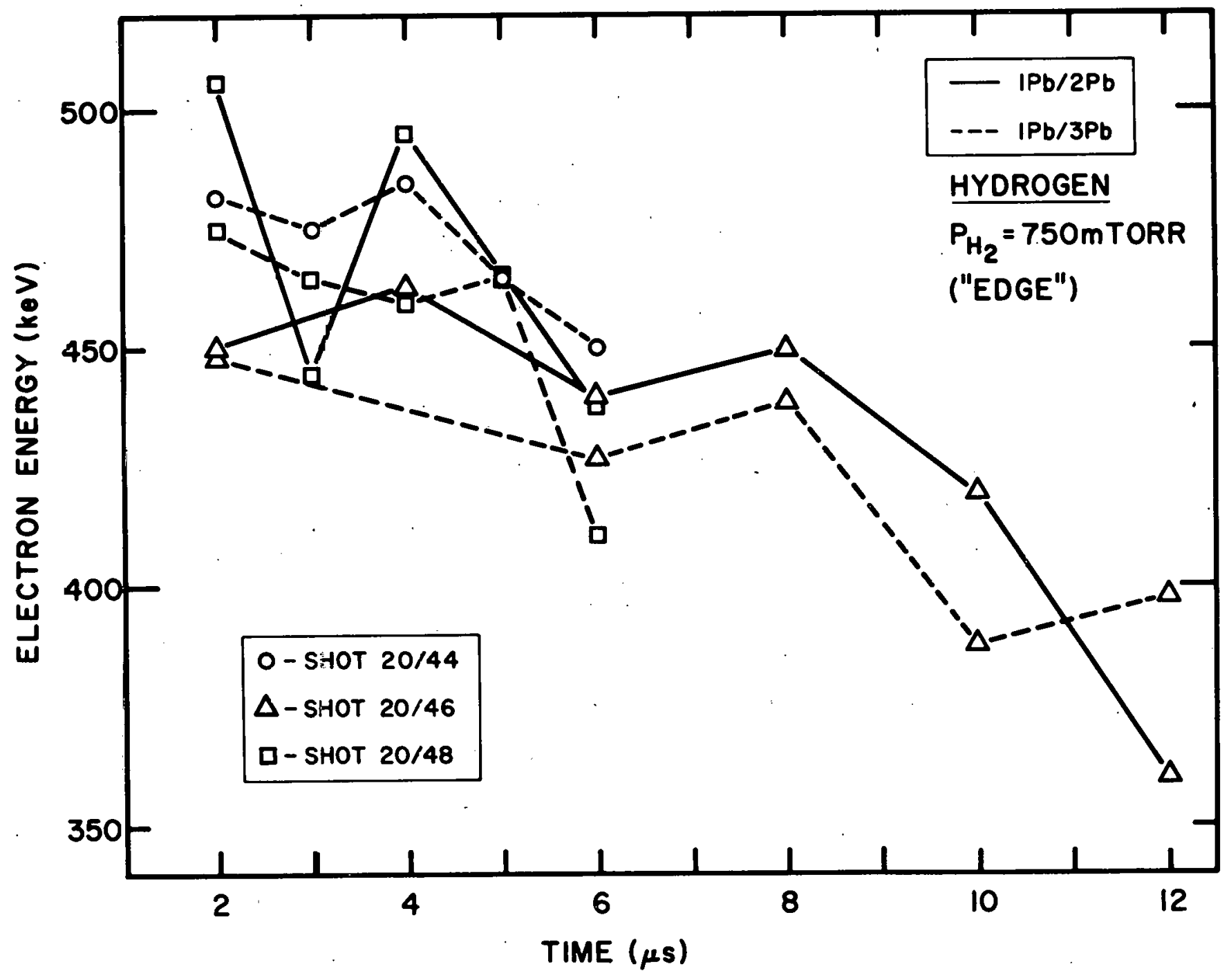




\section{FIGURE $4-30$}

Average electron energy as a function of time for an electron ring confined in a backfill of 410 mrorr hydrogen and $6 \mathrm{mTorr}$ argon; target located at edge of ring current distribution. 


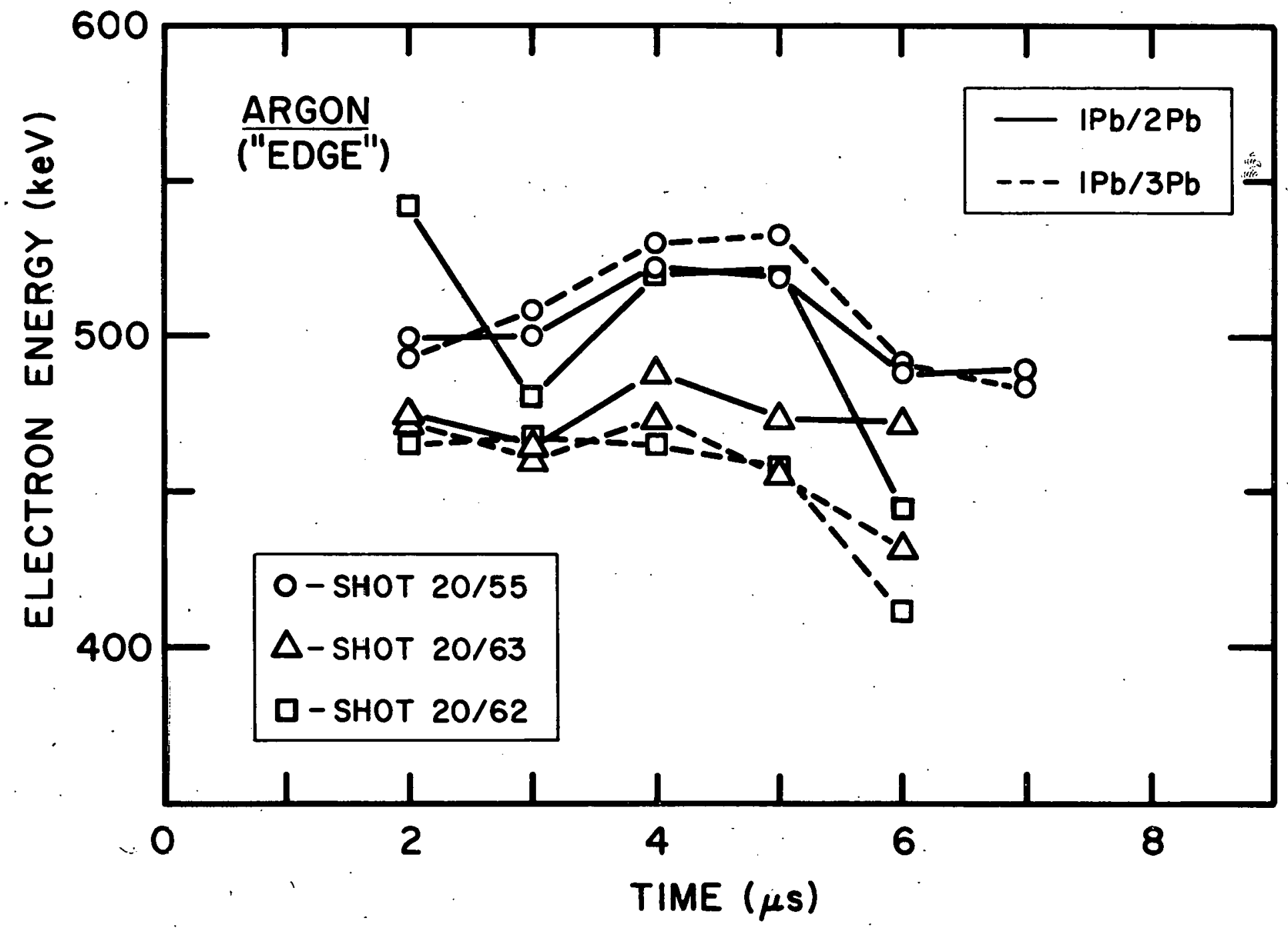


In all cases, the initial ring electron energy is seen to be about $500 \mathrm{keV}$, within the fluctuations of diode voltage and the accuracy of the measurement (see section 4.7). A qualitative inspection of the data of Figures $(4-27)-(4-30)$ shows--within the statistical accuracy of the measurement--a fairly uniform, monotonically-decreasing ring electron energy as a function of time. No catastrophic changes in average electron energy are seen to occur, as might accompany the onset of instability, and, overall, the time-dependence of the average electron energy can be well-approximated by a straight-line fit to the data.

From the curves fitted to the data for the different experimental conditions, one obtains the values for the average time rate of change of the average ring electron energy shown in Table 4-1.

\subsection{Experimental Error Analysis}

The final data reduction consists of taking the ratios of the oscilloscope trace deflections as a function of time and then using the curves of Figure (4-17) to obtain the average ring energy. Into this process must be folded the various experimental errors implicit in such a measurement, in addition to the overall code errors (shown to be $\sim 18-48$ in Section 4.4 .6 ). First, the 
TABLE $4-1$

Results of Experimental Measurements of du./dt.

$$
\begin{array}{lc}
\text { Machine Parameters } & \begin{array}{c}
\mathrm{dU} / \mathrm{dt} \\
(\mathrm{keV} / \mathrm{\mu sec})
\end{array} \\
\mathrm{P}_{\mathrm{H}_{2}}=450 \text { mTorr, } \mathrm{P}_{\mathrm{N}_{2}}=15 \mathrm{mTorr} \text { (edge) } & -7 \pm 3 \\
\mathrm{P}_{\mathrm{H}_{2}}=750 \text { mTorr, } \mathrm{P}_{\mathrm{N}_{2}}=15 \mathrm{mTorr} \text { (edge) } & -6 \pm 3 \\
\mathrm{P}_{\mathrm{H}_{2}}=450 \text { mTorr, } \mathrm{P}_{\mathrm{N}_{2}}=15 \mathrm{mTorr} \text { (center) } & -2 \pm 3 \\
\mathrm{P}_{\mathrm{H}}=410 \text { mTorr, } \mathrm{P}_{2}=15 \text { mTorr, } & -2 \pm 3 \\
\mathrm{P}_{\mathrm{Ar}}=40 \text { mTorr (edge) } &
\end{array}
$$


accuracy with which the thicknesses of the various absorbers were measured was $\pm 0.08 \mathrm{~mm}$, using a machinist's micrometer. Folding this error into the detector signals was found to incur a maximum error of about 18 for all three thicknesses of absorber used in the measurement. Therefore, the accuracy of the curves of Figure (4-17) is largely unaffected by this source of error. On the other hand, the primary contributors to the overall experimental uncertainty in this measurement are as follows $(E \sim 500 \mathrm{keV})$ :

\section{Source of Error}

i. Oscilloscope Trace Reading Accuracy

(i) Statistical Fluctuations

(ii) Baseline offset $( \pm 0.5 \mathrm{~mm})$ :

$$
\begin{aligned}
& 1-3 \mu \mathrm{sec} \sim 2-38 \\
& \text { End of Trace }-58
\end{aligned}
$$

2. Relative Detector Calibration Error overall Uncertainty
Relative Error $5-88$ 
of the measurement.

Better accuracy may be claimed for the measurements of the slopes of the energy-versus-time data, however. In this case, the only contributing factors to the uncertainty are trace fluctuations, baseline misalignment, and the trace reading error. Furthermore, the accuracy improves statistically as more data from each shot, and from independent shots, are included in computing $\mathrm{dU} / \mathrm{dt}$. Thus, if 4-6 shots are analyzed in determining du/dt from the data, then slope errors of the order of 3z-5z may be expected.

4.8 Average Ring Electron Energy Loss Rate Analysis

In this section, a comparison is made between the measured average electron energy loss rates and the calculated electron energy changes due to

(1) collisional energy loss with the background gas, and

(2) the accompanying "betatron" energy gain of the (inductive) ring electrons brought about by the decay of the axial magnetic field.

\subsubsection{Electron Energy Loss Rates}

The two primary classical energy loss mechanisms of the electrons as they pass through the background fill 
of gas are collisional ionization and Bremsstrahlung. For the numerical purposes of this work, use was made of the computations of Pages, et al. $9^{91}$ in which

(1) the ionization energy loss formulation is the relativistic, quantum-mechanical treatment of the problem by Bethe, 92

(2) the Bremsstrahlung energy loss is derived from the formulae of Koch and Motz. 93

For a $500 \mathrm{keV}$ electron travelling through a gas admixture of partial pressures of hydrogen $\left(\mathrm{P}_{\mathrm{H}_{2}}\right)$, nitrogen $\left(\mathrm{P}_{\mathrm{N}_{2}}\right)$, and argon $\left(\mathrm{P}_{\mathrm{Ar}}\right)$, the energy loss rate may be expressed as,

$$
\begin{aligned}
(d u / d t)_{\text {GAS }}= & -\left(12.9 \mathrm{P}_{\mathrm{H}_{2}}+77.1 \mathrm{P}_{\mathrm{N}_{2}}+90.0 \mathrm{P}_{\mathrm{Ar}}\right) \\
& \times 10^{-3}(\mathrm{keV} / \mu \mathrm{s})
\end{aligned}
$$

where the partial pressures are expressed in mTorr.

The experimentally-measured partial pressures actually used in Equation (4.13) had to be corrected for the "sweeping" action of the hydrogen in the vacuum pumping system which, for admixtures of 208 or less, 94 tends to reduce the partial pressures of the heavier gases and raise the relative pressure of the hydrogen. Experimentally, each gas pressure was established in the vacuum vessel by itself, and then all manifold release 
valves opened to form the admixture. The partial pressure corrections due to the differences in pumping speeds of the various gases were performed in a manner similar to that of Phelps. ${ }^{95}$ The data for the partial pressures obtained, along with the associated contributions to electron energy losses, are shown in Table 4-2. Typically, the errors in gas pressure measurements are about $10 \%$ in this pressure range.

\subsubsection{Betatron Acceleration}

In these experiments, we generate rings of intense electron current which have self-inductances and related self-magnetic fields. By Lenz's Law, changes in either the magnetic flux encircled by the ring or the current circulating in the ring will be met by electromagnetic forces which accelerate the circulating charge in a way to resist that change. This effect is usually called "inductive" or "betatron" acceleration. 96

Betatron acceleration effects may be applied in quantitative terms as follows: the average magnetic flux, $\phi$, linked by a single-turn loop of current, I, and inductance, $L$, is

$$
\phi=\mathrm{LI} \text {. }
$$

Taking time derivatives, we have 
TABLE $4-2$

Corrected Gasfill Partial Pressures and Fast Electron Energy Loss Rates.

RUN I

$$
\mathrm{P}_{\mathrm{H}_{2}}=450 \text { mTorr } \quad 450 \text { mTorr } \quad-6
$$

$\mathrm{P}_{\mathrm{N}_{2}}=15$ mTorr

4 mTorr

$\frac{-0.4}{-6.4}$

$\mathbf{P}_{\mathrm{H}_{2}}=750$ mrrorr

750 m'Torr

$-9.8$

RUN II

$$
\begin{aligned}
& \mathrm{P}_{\mathrm{N}_{2}}=.15 \mathrm{mTorr} \\
& \frac{-0.5}{-10.3} \\
& \mathrm{P}_{\mathrm{H}_{2}}=410 \text { mTorr } \\
& -5.4
\end{aligned}
$$

RIN TTT

$$
\begin{aligned}
& \mathrm{P}_{\mathrm{IN}_{2}}=15 \text { mTorr } \\
& \mathrm{P}_{\mathrm{Ar}}=40 \text { mTorr }
\end{aligned}
$$$$
4 \text { mTorr }
$$$$
-0.35
$$

6 mTorr

$$
\frac{-0.54}{-6.3}
$$




$$
\frac{\partial \phi}{\partial t}=L \frac{\partial I}{\partial t}+I \frac{\partial L}{\partial t}
$$

Thus, from Faraday's Law for a ring of constant diameter, $r_{0}$, a charged particle moving around the ring with an initial velocity $v_{\theta}=\beta_{\theta} c$ will gain energy at the rate

$$
\frac{d U_{\theta}}{d t}=\left(2 \pi r_{0} E_{\theta}\right) \frac{\beta_{\theta} c}{2 \pi r_{0}}=\frac{-\beta_{\theta} c}{2 \pi r_{0}}(L \dot{I}+I \dot{L})
$$

where the presumption has been made that $\beta_{\theta}$ is constant. This assumption will be justified in the experimental results.

Expressions for current and inductance depend upon the choices for ring geometry. Furthermore, the experimental measurements of the ring self-field are made on axis at the center of the ring, and the magnetic field measured by the probes is a superposition of the diamagnetic field from the ring plus the opposing magnetic field from the counter-induced currents in the conducting liners. An analytic expression relating $I(t)$ to $\mathrm{B}_{z}(t)$ (as measured on axis at the axial center of the electron ring) parameterized to the axial length of the circulating current distribution has been obtained by woodall, et al. 97 This treatment includes both non- 
uniform, axial current distribution (cosine ${ }^{2}$ ) effects, as well as the wall-induced "image fields". To an accuracy of $\sim 18$ of the actual calculated values, this relationship may be expressed in short form as

$$
I(t)=(10.82+(0.693) \Delta z(t)) B_{z}(t)
$$

where $B_{z}(t)$ and $\Delta z(t)$ are the axial, centrally-measured ring magnetic self-field (Gauss) and the current distribution FWHM (cm) as a function of time, respectively. The radial current distributions for the rings trapped in the cornell Experiments have not been measured, although such measurements have been performed on the comparatively weaker $(\zeta<58)$ E-layers confined in the Livermore Astron. 98 However, if we approximate the rings by a cylindrical annulus of current with a rectangular cross-section, as shown in Figure (4-31), then, following Terman, 99 we máy express the self-inductance of the ring as

$$
L=\frac{g r_{0}{ }^{2}}{6 r_{0}+3 \Delta 8+10 \Delta r} \mu \mathrm{H}
$$

For a coil of the dimensions we will be considering $\left(r_{0}-9 \mathrm{~cm}, \Delta z=10-20 \mathrm{~cm}, \Delta r=5-15 \mathrm{~cm}\right)$, the constant, 


\section{$-268-$}

FIGURE 4-31

Geometric model of electron ring used in loss rate analysis. 


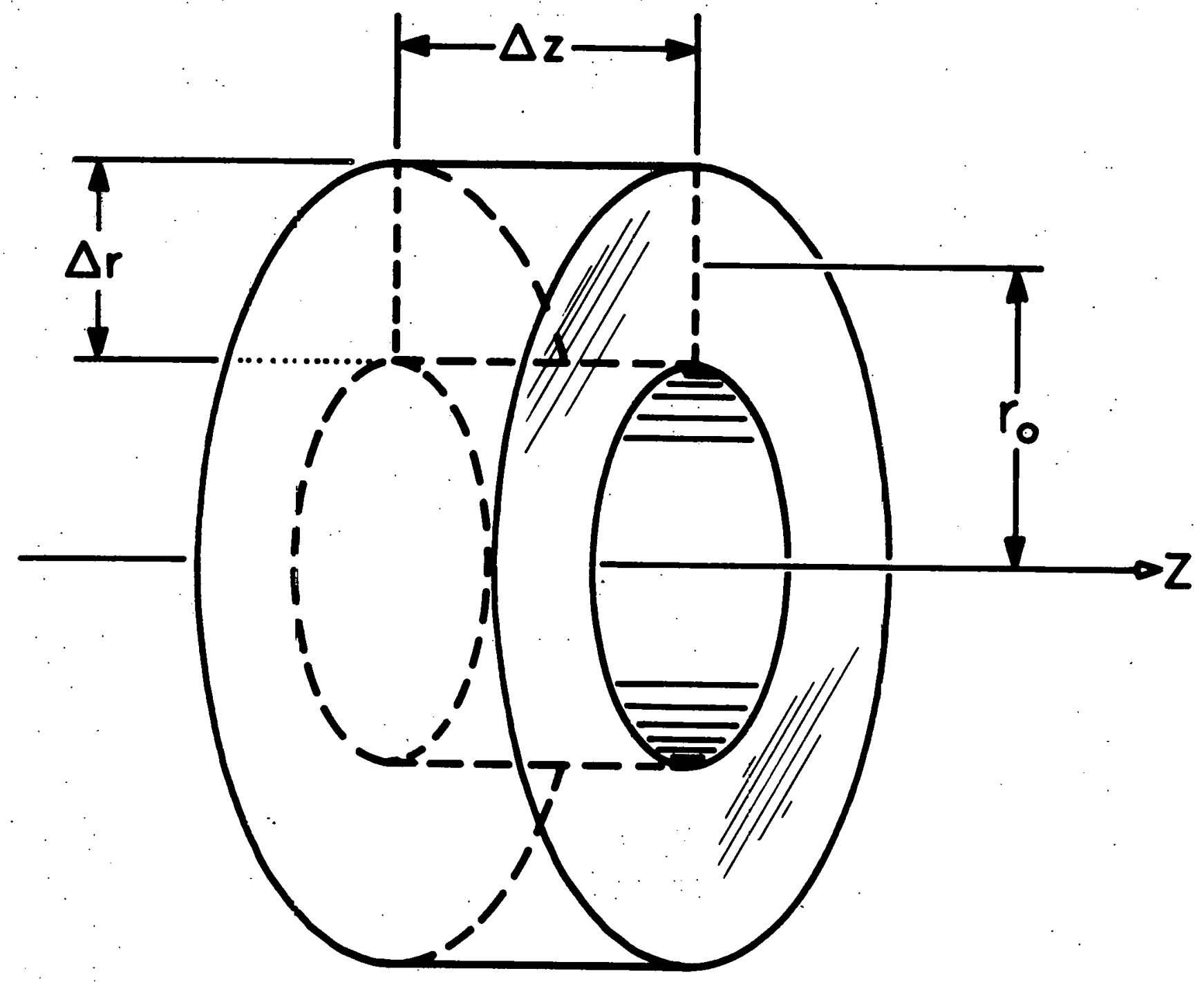

1
a
o

FIGURE 4-31 
9, has a value of $2.7 \times 10^{-7}$. This relationship for the self-inductance is accurate to within $1 \%$ for the assumed current distribution. As discussed in Section 3.4.1, the ring self-inductance expression, Equation (4.18), must be corrected for the effects of inductive coupling to the conducting liners. The experimental data of Figures $(3-12)-(3-16)$ and $(3-17)-(3-19)$ indicate that the axial current distribution FWHM is about $20 \mathrm{~cm}$ prior to the onset of axial lengthening of the electron ring (this axial lengthening was found 100 to proceed at a rate of about $4 \mathrm{~mm} / \mu \mathrm{sec})$, and increases to no more than $25-30 \mathrm{~cm}$ during the $10-12 \mu \mathrm{sec}$ ring lifetimes of these experiments. Under these conditions, this inductive correction term is about 10 - 158 of the selfinductance of the short, fat electron rings.

Expressions (4.17) and (4.18) were substituted into Equation (4.16) to calculate the betatron energy gain per unit time as a function of ring geometry, initial ring axial diamagnetic field strength, and the axiallycentered value of the rate of change of magnetic field, $\partial B_{z} / \partial t$ (obtained by averaging over all shots taken at each set of parameters, $\underline{i} \cdot \underline{e} .$, between 5 and 10 data points). The results of the calculation are plotted in Figure (4-32) as a function of time after injection. 


\section{FIGURE 4-32}

Betatron energy gain per electron as a function of time for various ring geometries and different axial magnetic field decay rates. 


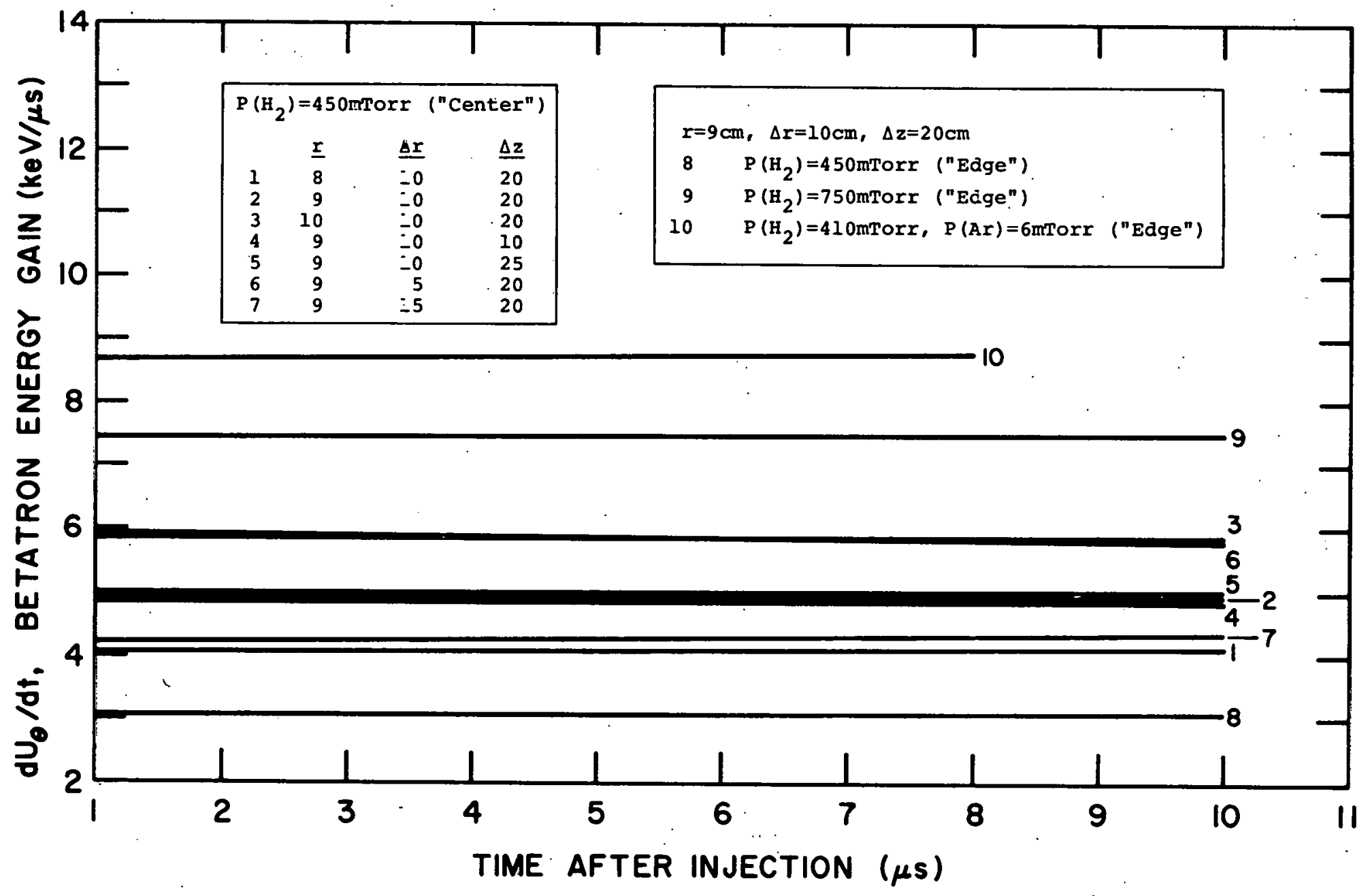

FIGURE 4-32 
Inspection of Figure (4-32) shows the energy gain to be a fairly constant function of time, reflecting both the effects of the characteristically linear axial diamagnetic field decay rate and the insensitivity of the ring's net inductance to axial lengthening.

The accuracy of the values computed for the Betatron acceleration may be estimated by considering the calculation's sensitivity to parameter variations. A summary of parameter variations tested and the percentrage relative error found in dU/dt is listed in Table 4-3. As shown in the table, compounding these independent errors yields a net error of $\pm 30 \%$ in the calculated values for $\mathrm{dU} / \mathrm{dt}$.

\subsubsection{Summary of the Experimental and Computational}

\section{Results:}

The net rate of change of ring electron energy may be obtained by summing the energy loss rates via ionization and Bremsstrahlung (Table 4-2) with the Betatron energy gain rates as calculated through Equation (4.16) for the four different sets of parameters studied in the experimental work. The results of these calculations, with a summary of the contributing energy loss/gain terms, is shown in Table 4-4.

From the experimental data, and Figure (4-18), we 
$-274-$

TABLE $4-3$

Sensitivity of Calculated du/dt Values to Ring Parameter variations.

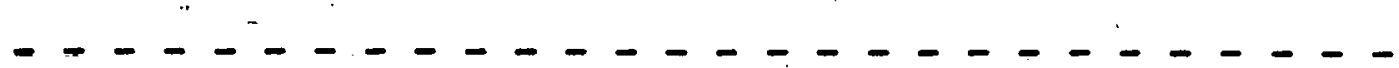

$$
\mathrm{du} / \mathrm{dt}
$$

Parameter Variation variation

$$
\begin{array}{lcc}
\mathrm{r}_{0} & \pm 108 & \pm 20 \% \\
\Delta z & \mathrm{z}_{0} / 2,2 z_{0} & \pm 1.68 \\
\Delta \mathrm{r} & \mathrm{r}_{0} / 2,3 \mathrm{r}_{0} / 2 & \pm 17 \mathrm{z} \\
\mathrm{\partial B}_{\mathrm{z}} / \partial t & \pm 208 & \pm 178 \\
\mathrm{~B}_{z 0} & \pm 208 & \pm 0.28 \\
& \text { Net Error: } & \pm 308
\end{array}
$$




\section{TABLE 4-4}

Summary of Ring Electron Energy Decay Rates.

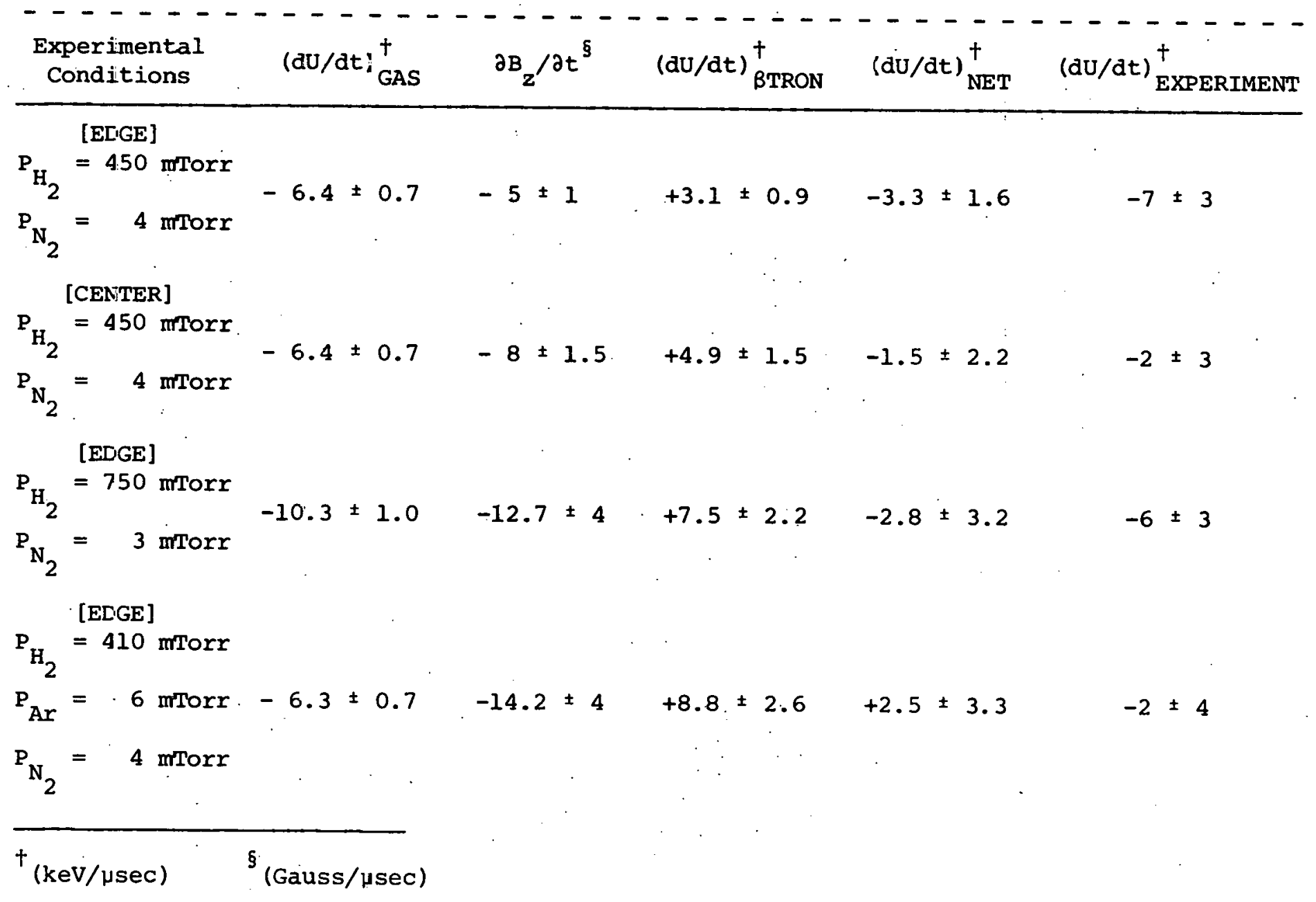


may also obtain a rough estimate of the predicted ring electron energy spread for the various experimental conditions. Examples of the predicted energy spreads are shown in Table 4-5. As may be seen, the actual energy differences obtained from the various absorber ratios are far less than the uncertainty, $\varepsilon$, in the overall measurement. Under three of the sets of experimental conditions, the energy predicted by the ratio of $1 \mathrm{~Pb} / 2 \mathrm{~Pb}$ exceeded that predicted by $2 \mathrm{~Pb} / 3 \mathrm{~Pb}$, which is contrary to the results shown in Figure (4-18), but which differences still fell within the error limitations of the experiment. Hence, for these cases, the percentage energy "spread", $\triangle E / E$, is limited by the accuracy of the measurement, or $\sim 10 \%$. For the case of the target located at the edge of rings trapped in $750 \mathrm{mTorr}$ hydrogen, $E(1 \mathrm{~Pb} / 2 \mathrm{~Pb})-\mathrm{E}(2 \mathrm{~Pb} / 3 \mathrm{~Pb}) \sim 20 \mathrm{keV}$, which is still far less than the uncertainty in the overall measurement. Therefore, under the circumstances of this experiment, we cannot determine the energy spread of the ring electrons except to say that it appears to be less than the uncertainty of the measurement technique itself. 


\section{TABLE 4-5}

Predicted Ring Electron Energy Spreads.

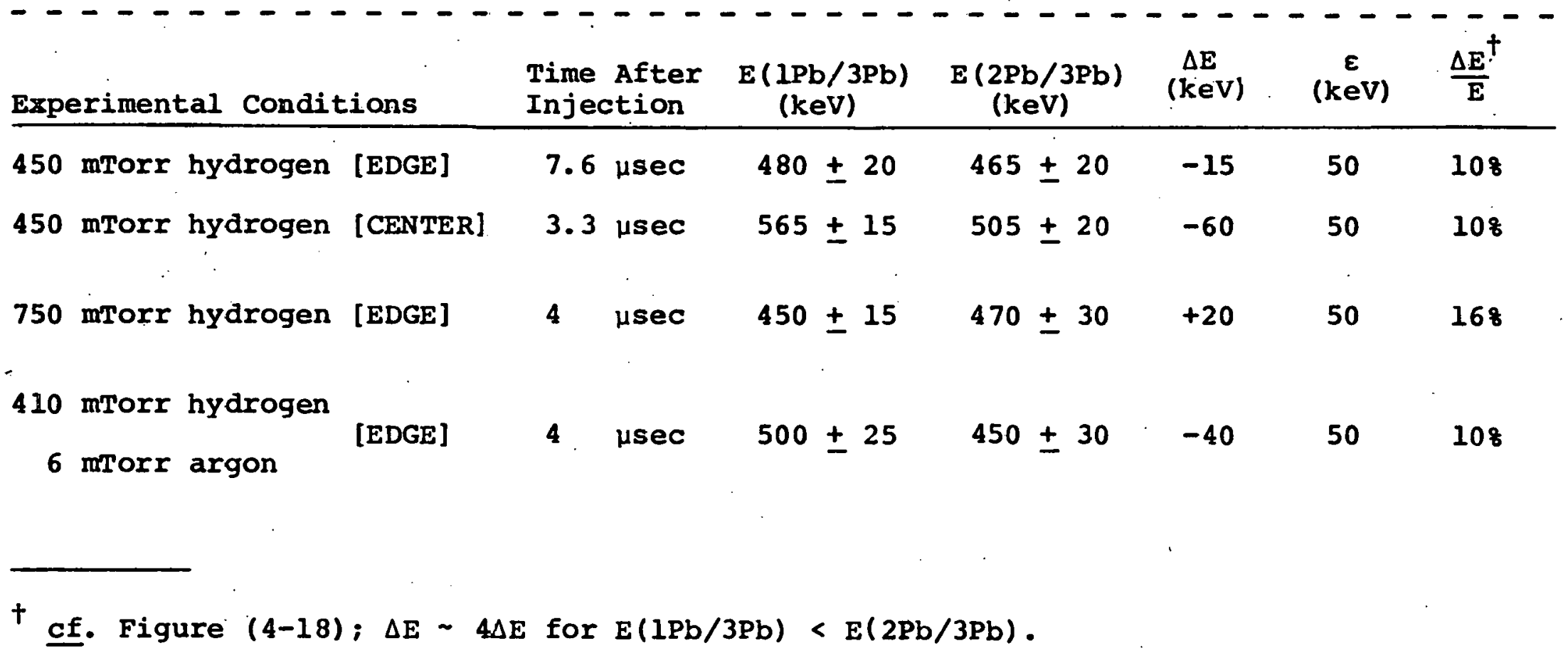

cf. Figure $(4-18) ; \Delta E \sim 4 \Delta E$ for $E(1 \mathrm{~Pb} / 3 \mathrm{~Pb})<\mathrm{E}(2 \mathrm{~Pb} / 3 \mathrm{~Pb})$. 


\subsection{Summary and Discussion of the Results}

\subsubsection{Discussion of the Experimental Findings}

Inspection of Table 4-4 shows an overall agreement, within experimental error, between the measured and the calculated rates of change of the average ring electron energy.

First, with a backfill of 450 mporr of hydrogen plus background nitrogen, we find, with the wire at the edge of the layer, the experimental measurement of the average ring electron energy to be larger than the calculated value. With the wire moved to the center of the current distribution, as expected, the rate of change of axial magnetic field increases--with a corresponding increase in Betatron acceleration--and fairly good agreement is observed between calculated and measured values for $d U / d t$.

With the target at the edge of the ring, increasing the backfill of hydrogen to 750 mTorr was expected to increase the collisional energy loss rate of the electrons in the gas, (dU/dt) GAS , to $-10 \mathrm{keV} / \mu \mathrm{sec}$ while enhancing the scattering loss of particles by a factor of 1.7. In fact, (dU/dt) ${ }_{B T R O N}$ is seen to increase by a factor of 2.5 giving the overall result, again, of a 
predicted (dU/dt) ${ }_{\text {NET }}$ which is lower than the experimentally-measured value, but which is still well within the permissible limits of error.

In the last set of parameters studied, the purpose of admixing the argon with the hydrogen was to bring about an enhanced scattering of the electrons without significantly altering the energy loss rates in the background gas. To this end, 420 mTorr hydrogen was mixed with 40 mTorr of argon (effective pressures of 410 and 6 mTorr, respectively, upon admixing). As indicated in Table 4-4, the (dU/dt) GAS losses remained virtually unchanged while (dU/dt) ${ }_{B T R O N}$ increased by about a factor of three. The result was a prediction of a net acceleration of the ring particles. In fact, some of the experimental data indicated possible net energy increases during the first microseconds of ring lifetime, but, when all data were taken into consideration, an overall energy loss rate of $-2 \mathrm{keV} / \mathrm{usec}$ was obtained. A net energy gain of $+2.5 \mathrm{keV} /$ usec as predicted is possible within the error of the measurement, but the average ring lifetime in admixtures of argon was not long enough (- 4-6 usec) to ascertain whether in fact such a gain could be taking place.

Note should also be made that, in no combination of 
experimental parameters, does the measured (or predicted) energy loss/gain rate exceed $7 \mathrm{keV} / \mu \mathrm{sec}$, or slightly more than 108 of $E_{0}$ over a typical ring lifetime of $10 \mu \mathrm{sec}$. Hence, the assumption in Equation (4.16) that the particle velocity, $\beta_{\theta} c$, remains fairly constant throughout the energy transfer process over the ring lifetime is justified.

Overall, the measured values of the average ring electron energy are quite easily in keeping with expectations $\underline{i} . \mathrm{e}_{.}, \mathrm{E}_{\mathrm{o}} \sim 500 \mathrm{keV}$, which is consistent with the energy of the injected beam inferred from the diode accelerating voltages. However, the experimental data consistently gave energy loss rates slightly in excess of the calculated values, but which were always in agreement within the uncertainties of both the measured and calculated values for (dU/dt) ${ }_{\text {NET }}$. Thus, the two principal conclusions to be drawn from these measurements are:

(1) that, in fact, the net average ring electron energy loss rates could be reasonably explained by simple, classical arguments, and

(2) no "anomalous" energy losses were apparent, indicating that instabilities either saturated at very low levels, or did not exist in the first place. 
4.9.2 Comparison with Theoretical Analyses of Microinstabilities.

To first order, the physical picture of the confined electron rings in our experiments is that of a collection of fast electrons streaming through a collisional background plasma in the presence of a background magnetic field. Therefore, a reservoir of free energy is certainly made available for the production of unstable plasma disruptions, such as the two-stream instability, for example. Hence the question arises as to why this apparently does not happen (at least at noticeable levels) under our experimental conditions. The overall stability of circulating currents in a plasma in a background magnetic field was considered theoretically by Striffler and Kammash. ${ }^{101}$ In their theoretical treatment, certain simplifications were nesessary:

(1) the self-fields of the rings were neglected;

(2) the curvature of the particles' trajectories was neglected, and

(3) to allow application of a localized planewave treatment of the problem, consideration was given only to perturbations having wavelengths much smaller than the characteristic dimensions of the rings. 
Employing a rectangular coordinate system in which $\overrightarrow{\mathrm{B}}_{0}$; the magnetic field, is parallel to $\hat{e}_{z}$, and the circulating beam current is along $\hat{e}_{y}$, we have, from condition (3), $\lambda_{\mathrm{X}} \ll \Delta \mathrm{r}$ and $\lambda_{\mathrm{z}} \ll \Delta \mathrm{z}$. A further assumption that $\lambda_{y} \sim 2 \pi v_{B} / l \Omega_{B}$ was made to reflect the expectation that, should instabilities arise in the presence of the magnetic field, they are most likely to appear at harmonics of the beam's cyclotron frequency $\left(V_{B}\right.$ is the beam's azimuthal speed, $\ell$ is the harmonic number, and $\Omega_{B}$ is the beam's cyclotron frequency).

In their calculations of the growth rate of the instability are included the effects of:

(1) collisions between plasma and neutral gas particles (since the plasma is formed by beam ionization of a neutral gas), and

(2) beam energy spread.

The plasma densities considered were those such that the plasma upper hybrid frequency, $\omega_{H}=\left(\omega_{p}^{2}+s r_{e}^{2}\right)^{1 / 2}$ (where $\omega_{p}$ is the electron plasma frequency and $\Omega_{e}$ is the plasma electron cyclotron frequency),

(i) coincided with, and

(ii) deviated from harmonics of the beam cyclotron frequency, i..e.., that the condition 


$$
\frac{\omega_{p}}{\Omega_{e}} \cong\left\{\frac{\ell^{2}+r^{2}}{r^{2}}\right\}^{1 / 2}
$$

was satisfied, where $\gamma$ is the relativistic mass factor. To include the effects of collisions, a plasma/ neutral particle momentum transfer collision frequency was incorporated into the plasma two-fluid equations. Correspondingly, the relativistic fluid equations were. used to describe the beam particles and their interaction with an electromagnetic wave. These equations. were linearized and combined to give a wave equation, from which a general dispersion relationship was obtained for the extraordinary mode.

Although such a linear analysis cannot meaningfully predict full quenching of the instability via collisions, one may obtain estimates for the magnitudes of the collision frequencies necessary to stabilize the mode, $\underline{i}$.e... cause the linear-theory growth rate to go to zero. Kammash and Striffler obtain relationships which imply stabilization under the conditions of,

(i) resonance and

(ii) non-resonance of the hybrid frequency and the $e^{\text {th }}$ beam harmonic.

A summary of these criteria and their application to the experimental conditions in RECE-Berta is presented in 
Table (4-6):

From the table, we see that, under our experimental conditions, collisional effects alone are predicted to be either marginally stabilizing or do not contribute to stabilization at all.

In order to consider the effects of beam energy spread (in the direction of the beam) on the growth of the instability, the beam was described using the relativistic Vlasov Equation in which the beam was represented as a "square" distribution in beam particle velocities centered around a monoenergetic value, $u_{\dot{y} B}$. The beam current density was calculated directly from the distribution function from which the linearized, perturbed current density was obtained. This result was then combined with the linearized fluid equations de-scribing the perturbation of the plasma about its equilibrium state to yield a general dispersion relationship.

In our experiments, the energy spread in the direction of the beam arises primarily from the angular spread of the electron trajectories as they circulate around the current ring. Based on particle orbits in a straight beam of density $\mathrm{n}_{e} \sim 5 \times 10^{9} \mathrm{~cm}^{-3}$ (corresponding roughly to a field-reversing ring) and $\gamma \sim 2$, we estimate $\theta_{\text {RMS }} \geq 30^{\circ}$. Thus; we have a velocity spread along the 


\section{TABLE 4-6}

Summary of Hybrid Mode Collisional Stabilization Criteria EXPERIMENTAL CONDITIONS :

$$
\begin{gathered}
\mathrm{B}_{0} \cong 150 \mathrm{G} \quad \mathrm{r}^{\prime}=1.88 \quad \mathrm{n}_{\mathrm{p}}^{\circ} \text { (MEASURED) } \cong 10^{12} \mathrm{~cm}^{-3} \\
\mathrm{n}_{\mathrm{e}} \cong 5 \times 10^{9} \mathrm{~cm}^{-3}
\end{gathered}
$$

$\Omega_{p} \equiv 2.6 \times 10^{9} \mathrm{rad} / \mathrm{sec}=$ Background Plasma Frequency $\Omega_{B} \cong \Omega_{e} / \gamma=1.4 \times 10^{9} \mathrm{rad} / \mathrm{sec}=$ Beam Cyclotron Frequency $\omega_{p} \cong$ Background Plasma Frequency $\omega_{B} \cong 2.9 \times 10^{9} \mathrm{rad} / \mathrm{sec}=$ Beam Plasma Frequency

$$
=\left\{\frac{4 \pi n_{e} e^{2}}{m_{e}}\right\}^{1 / 2}
$$

COLLISIONAL EFFECTS:

$$
\begin{aligned}
& \text { A) Non-Resonance: } \ell<\gamma: \frac{\omega_{p}^{2}}{\Omega_{p}^{2}}<\frac{\omega_{p}^{\star 2}}{\Omega_{p}^{2}} \\
& =\frac{4 l^{2}\left(l^{2}+\gamma^{2}\right)-\left(\gamma^{2}-l^{2}\right)}{\gamma^{2}\left(\gamma^{2}-l^{2}\right)} \text { For Stability } \\
& \text { Berta Conditions: } n_{p}^{0}<n^{*}=4.5 \times 10^{9} \\
& \text { for stability } \\
& \ell>\gamma: \text { Always Stability }
\end{aligned}
$$




\section{TABLE 4-6 (Continued)}

B) Resonance: $\nu_{\mathrm{eN}}^{0}>\nu_{\mathrm{eN}}^{*}=\frac{3 \sqrt{3}}{2} \frac{\Omega_{\mathrm{p}}}{\gamma} \frac{2 \ell^{2}}{\ell^{2}+\gamma^{2}}\left\{\frac{\ell^{2}-\gamma^{2}}{2 \ell} \frac{\omega_{\mathrm{B}}^{2}}{\Omega_{\mathrm{B}}^{2}}\right\}^{1 / 3}$

For Stability

$$
\begin{aligned}
& \text { Berta Conditions: } P\left(\mathrm{H}_{2}\right)=450 \text { mTorr, } \nu_{\mathrm{eN}}^{\circ} \sim 1.5 \times 10^{9} \\
& \sec ^{-1} \\
& \mathrm{P}\left(\mathrm{H}_{2}\right)=900 \text { mTorr, } \nu_{\mathrm{eN}}^{0}-3 \times 10^{9} \\
& \sec ^{-1} \\
& \text { l. } \nu_{\mathrm{eN}}^{\star}\left(\sec ^{-1}\right) \\
& 22.2 \times 10^{9}+\text { (Marginally Stabilizing) } \\
& 37.8 \times 10^{9} \\
& 41.1 \times 10^{10} \\
& 5 \quad 1.3 \times 10^{10} \\
& 6 \quad 1.4 \times 10^{10} \\
& 7 \quad 1.6 \times 10^{10} \\
& 8 \quad 1.8 \times 10^{10} \\
& 9 \quad 1.8 \times 10^{10} \\
& 10.1 .9 \times 10^{10}
\end{aligned}
$$


direction of the beam of $\Delta V / V \sim 10-15 \%$. For such a "large" velocity spread (which, following singhaus, 102 the authors define as $\Delta V$ such that $\left.\tau=\left(\omega_{p} \Delta V / \omega_{b} V\right)>1\right)$, stability criteria were derived, as before, for beam cyclotron frequency harmonics above and below the plasma electron cyclotron frequency (for our experimental conditions, $\tau \sim 6$ ). These criteria are summarized in Table (4-7).

Inspection of Table (4-7) indicates that, since the measured plasma densities were always of the order of $10^{12} \mathrm{~cm}^{-3}$ under our experimental conditions (cf. Chapter 3), we see that the theory predicts stability for the first 21 harmonics of the beam cyclotron frequency (only data for the first ten are listed in the table).

In the event that $B_{0} \rightarrow 0$, the analysis of striffler and Kammash becomes identical with that of Singhaus, in which the instability makes the transition from a partially-electromagnetic, partially-electrostatic wave interaction to a purely electrostatic two-stream coupling between the beam and the plasma. In this situation, Singhaus predicted stabilization of the two-stream interaction (for a "large" velocity spread in the beam, as defined above) if the criterium that. 


\section{TABLE $4-7$}

Sumary of Hybrid Mode Energy Spread Stabilization Criteria

\section{ENERGY SPREAD}

Resonant Interaction

A) $\ell<\gamma\left\{\frac{\omega_{p}}{\Omega_{p}}\right\}^{2}>\left\{\frac{\omega_{p}^{*}}{\Omega_{p}}\right\}^{2}=\frac{\gamma^{2}-\ell^{2}}{\gamma^{2}\left(\gamma^{2}-1\right)}$

$$
x\left\{1+\frac{3}{2} \frac{\gamma^{2}-1}{l^{2}} \frac{\omega_{B}^{2}}{\Omega_{B}^{2}}\right\} \text { For stability }
$$

Berta Conditions :

$$
n_{p}>n^{\star}=6 \times 10^{9} \mathrm{~cm}^{-3} \quad \text { For Stabilization }
$$

B) $\ell>\gamma \quad \omega_{p}>\omega_{p}^{*}=\Omega_{p}\left\{\frac{\ell^{2}-\gamma^{2}}{\gamma^{2}}\right\}^{1 / 2}$

Berta Conditions :

$$
\begin{array}{llll}
\ell & \mathrm{n}_{\mathrm{CRIT}}\left(\mathrm{cm}^{-3}\right) & 6 & 2 \times 10^{10} \\
2 & 2.9 \times 10^{8} & 7 & 7 \times 10^{10} \\
3 & 3.4 \times 10^{9} & 8 & 3.8 \times 10^{10} \\
4 & 7.7 \times 10^{9} & 9 & 4.7 \times 10^{10} \\
5 & 1.3 \times 10^{10} & 10 & 5.9 \times 10^{10} \\
& \mathrm{n}_{\mathrm{p}}^{0}>\mathrm{n}_{\mathrm{CRIT}} \text { for } 2 \leq \ell \leq 21
\end{array}
$$




$$
\frac{\Delta v}{V}>0.76 \frac{\omega_{B}^{2}}{\omega_{p}{ }^{v}{ }_{c}}, \omega_{B}^{2}=\frac{4 \pi n e^{e^{2}}}{m_{e^{\gamma}}}
$$

were satisfied. (This criterium was corraborated experimentally by Böhmer, et al.).103 Under our experimental conditions, this theory predicts stabilization for $\Delta V / V \geq 108$, which is comparable with our estimated value of $\Delta v / V \sim 10-15 \%$.

In light of these results--granted the simplifications and approximations involved in the various data-we see that it is not unexpected that instabilities might not play an important role in ring behaviour under our experimental conditions.

4.0 Extension and Application of Energy Measurement to Nanosecond Time Scales

There is currently considerable interest ${ }^{104}$ in the plasma physics community in the amount of energy deposited in a gas/plasma following the injection of high-current, relativistic electron beams into the medium in question. A convenient technique for measuring beam energy deposition would be to monitor the beam energy degradation as a function of penetration distance into the gas/plasma. Multi-channel absorption spectroscopy, 
as described in this chapter, would seem to be a viable technique for such a measurement in the energy range of 200-800 keV. A wire target could be located at various axial positions along the beam path and the spatial and temporal behaviour of the beam energy determined via observation of the target Bremsstrahlung.

The diagnostic described in this thesis, however, was concerned with events taking place in 10-20 $\mathrm{usec}$ time scales, and could therefore utilize the high-sensitivity sodium iodide detectors with fairly slow (250 ns) decay times for the measurements in question. On the other hand, the time scales of many beam-plasma experiments is of the order of 50-500 ns. (often limited by the pulsewidth of the beam). Thus, to apply the multichannel technique, one must resort to faster scintillators such as solid plastics NE102 or Pilot B.

Although no energy measurements were performed experimentally in this work using plastic scintillators, the computer codes described in Chapter 4 were adapted to the faster detectors and the ratios of the intensity integrals as a function of energy re-calculated. Operationally, this involved only a recomputation of the crystal detection efficiency as a function of energy. For reference, the results of these calculations are 
presented here.

The scintillator modelled for the calculations was undoped Pilot B. Because the photoabsorption edge of Pilot B (a pure hydrocarbon) is around 250-300 volts, the $\mathrm{X}$-ray interaction process which predominates in the energy range of 100-1000 keV is strictly compton Scattering. Hence, one may expect significantly lower detection efficiencies from such a scintillator. In order to enhance the detection efficiencies of plastic scintillators, it is customary to use fairly large size detectors in order to increase the number of $x$-ray interactions within the crystal.

The crystal geometry used in the calculations is illustrated in Figure (4-33). The scintillator itself is a $10.1 \mathrm{~cm} \times 10.1 \mathrm{~cm}$ right-cylinder of pilot B plastic, sheathed on its forward face and surrounding sides by a $0.81 \mathrm{~mm}$ thick aluminum housing. The rear face of the crystal is assumed mated to a conical lightpipe which tapers to match the front face of a photomultiplier tube. The photon beam is, as before, collimated to the center of the front face of the plastic with an effective aperture of $1.27 \mathrm{~cm} \times 1.27 \mathrm{~cm}$.

The resulting crystal efficiency as a function of energy is shown in Figure (4-34). As expected, the 
$-292-$

FIGURE $4-33$

Collimation geometry used for calculation of Pilot B crystal detector efficiency. 


\section{LEAD}

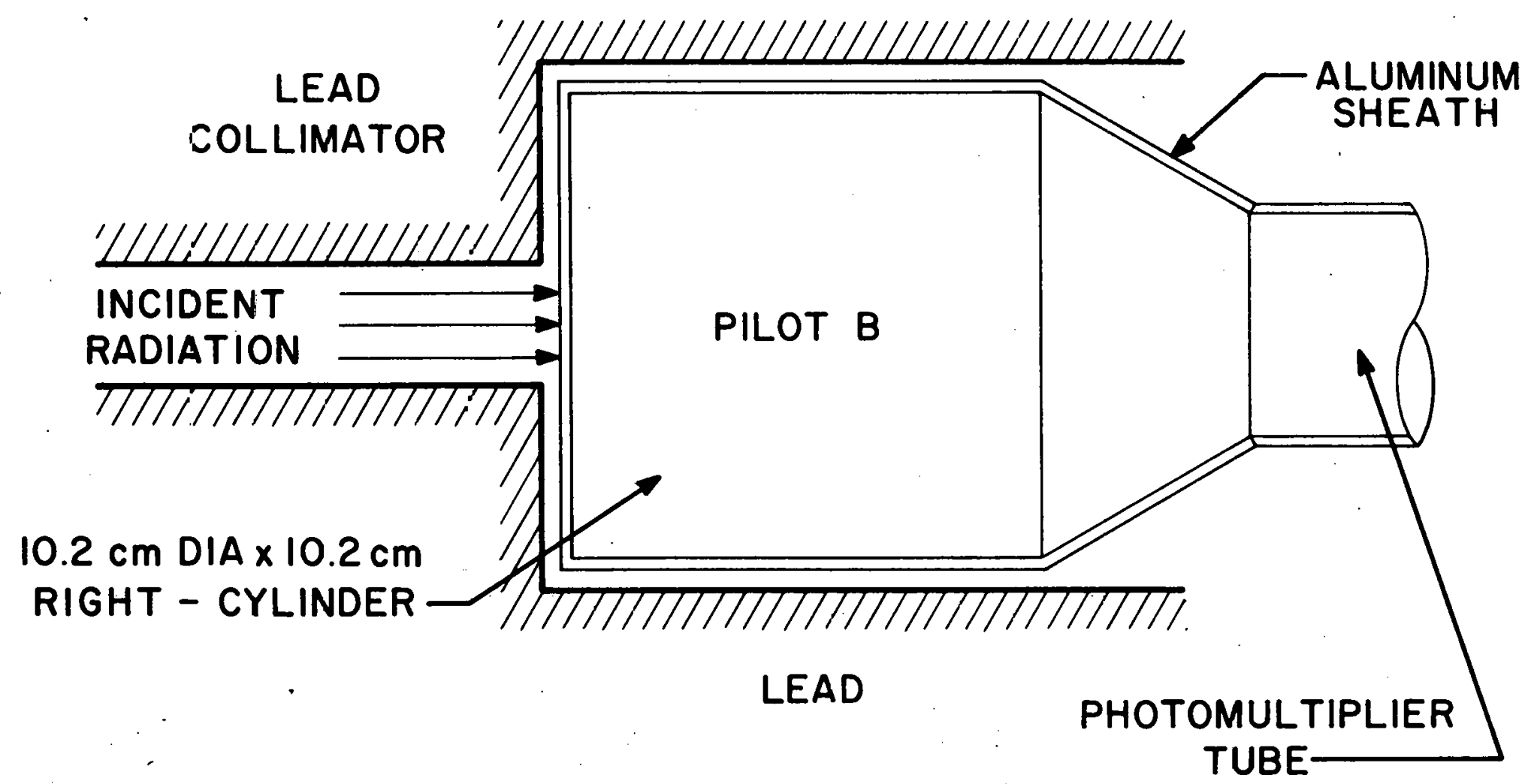


FIGURE 4-34

X-ray detection efficiency for a right-cylindrical

Pilot B crystal $10.1 \mathrm{~cm}$ in diameter and $10.1 \mathrm{~cm}$ long. 


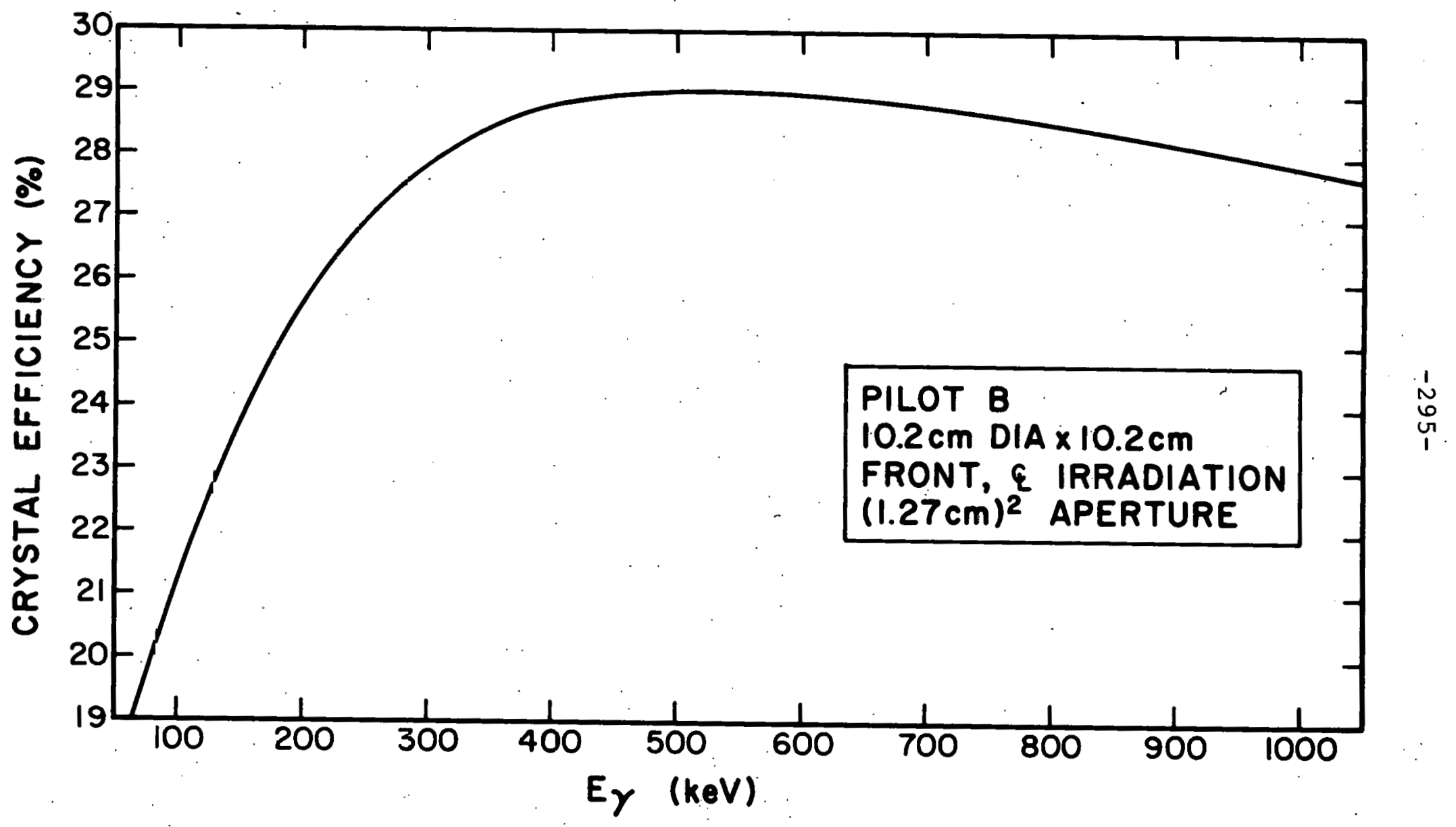

FIGURE 4-34 
efficiency is seen to be lower than the sodium iodide crystal efficiency, and is almost "flat" in the energy range of $200 \mathrm{keV}<E_{0}<1000 \mathrm{keV}$, ranging from 25.58 to 27.58 with a peak in the neighborhood of $500 \mathrm{keV}$. This maximum results from the peak in the total compton absorption cross-section at $500 \mathrm{kev}, 105$ i.e... that photon energy at which the maximum amount of energy may be imparted to the recoil crystal electron. This detector efficiency was inserted into the calculation for the integral intensities as a function of energy and absorber thickness, Equation (4.2). In a fashion identical to that described earlier, intensity ratios as a function of energy and absorber thickness were then obtained. A plot of these ratios as a function of electron energy and parameterized to absorber thickness is shown in Figure (4-35). Its overall features are seen to be quite similar to the analogous plot for sodium iodide, Figure (4-17).

Finally, a plot of predicted electron energy spread based on ratios of various thicknesses of absorbers is shown in Figure (4-36) for a diffuse and a unidirectional electron beam. As in Figure (4-18), the idealized case of a beam consisting of two delta-functions in energy symmetrically spaced about $\mathrm{E}_{0}=500 \mathrm{keV}$ was considered. 
FIGURE $4-35$

Families of curves depicting Pilot B detector signal ratios as a function of primary electron energy parameterized to the various thicknesses of absorber used in the experiment. 


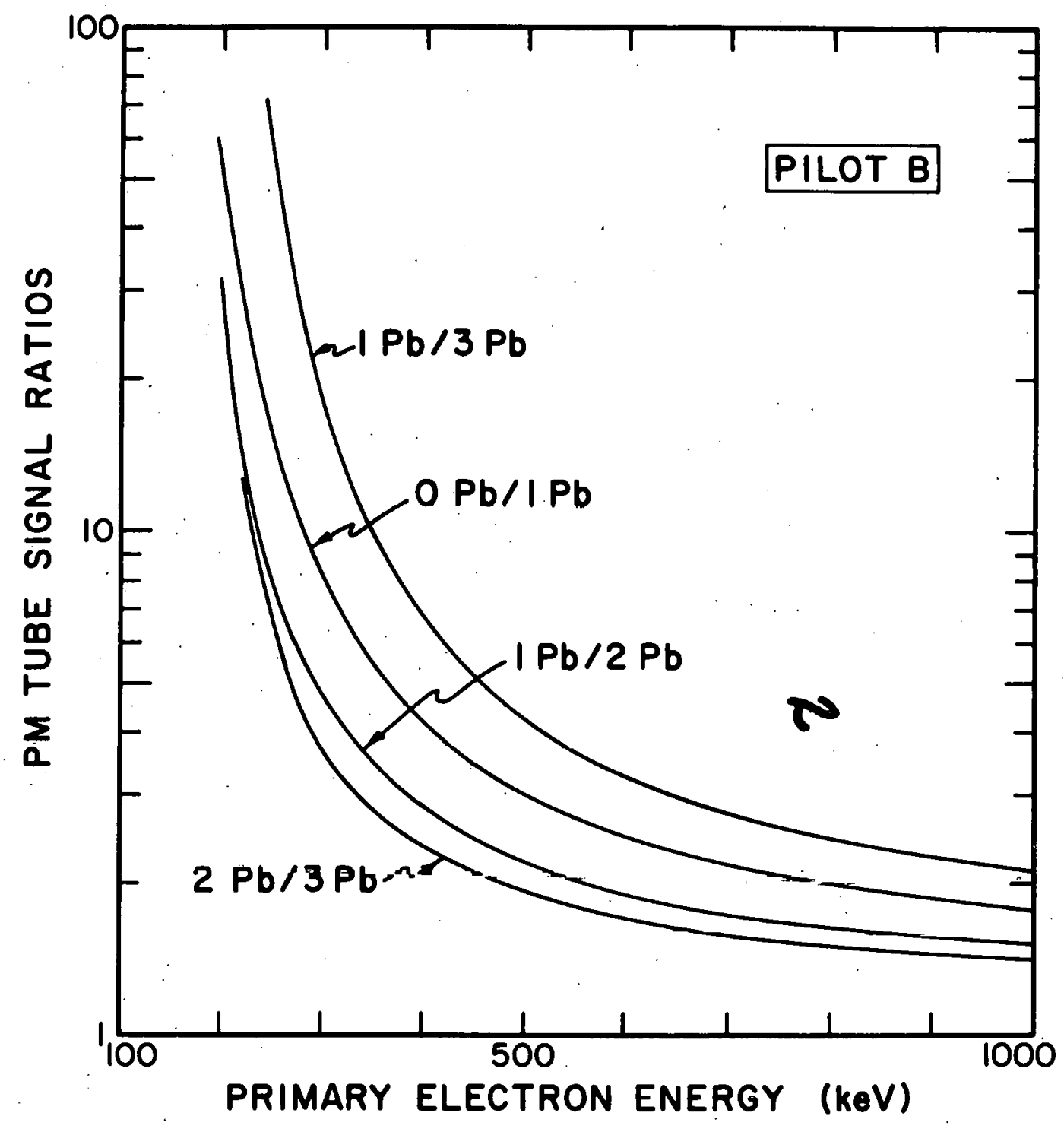


FIGURE $4-36$

Primary electron energy as predicted by various Pilot $B$ detector signal ratios having different absorber combinations for a monodirectional and diffuse beam. 


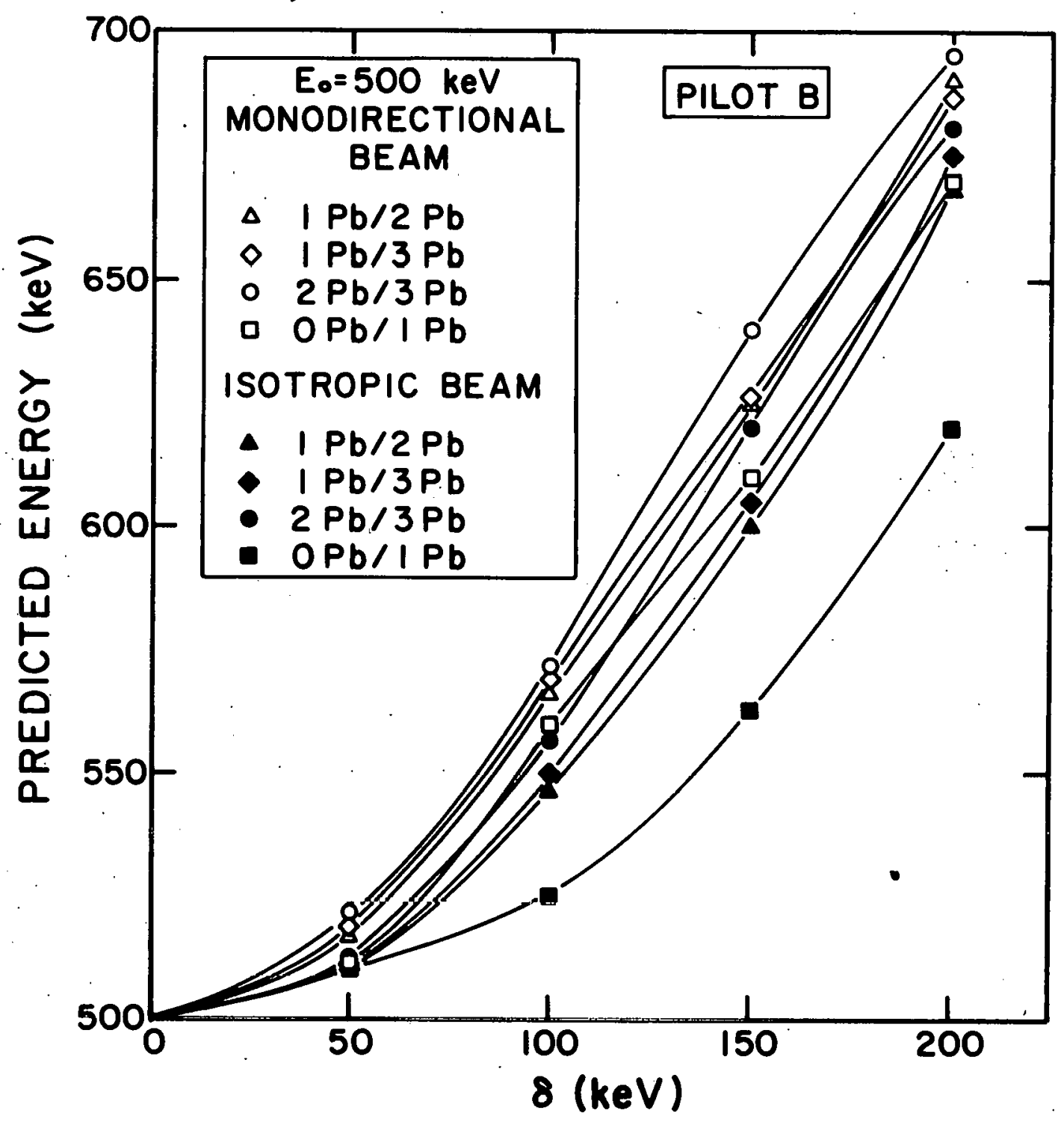


Overall, the plots of signal ratios vs electron energy, and predicted energy spread parameterized to signal ratios for the plastic detectors are quite similar to those plots for the sodium iodide detectors.

Unfortunately, owing to the lower crystal detection efficiency at all energies of interest here, use of such detectors would undoubtedly also lead to decreased signal levels. At any rate, the "usable" range of electron energies in which the technique may be effectively applied remains between 250 and $700 \mathrm{kev}$.

For reference, a listing of the code-generated crystal efficiencies and detector signal ratios as a function of electron energy for the Pilot B detector is presented in Appendix III.4. 